
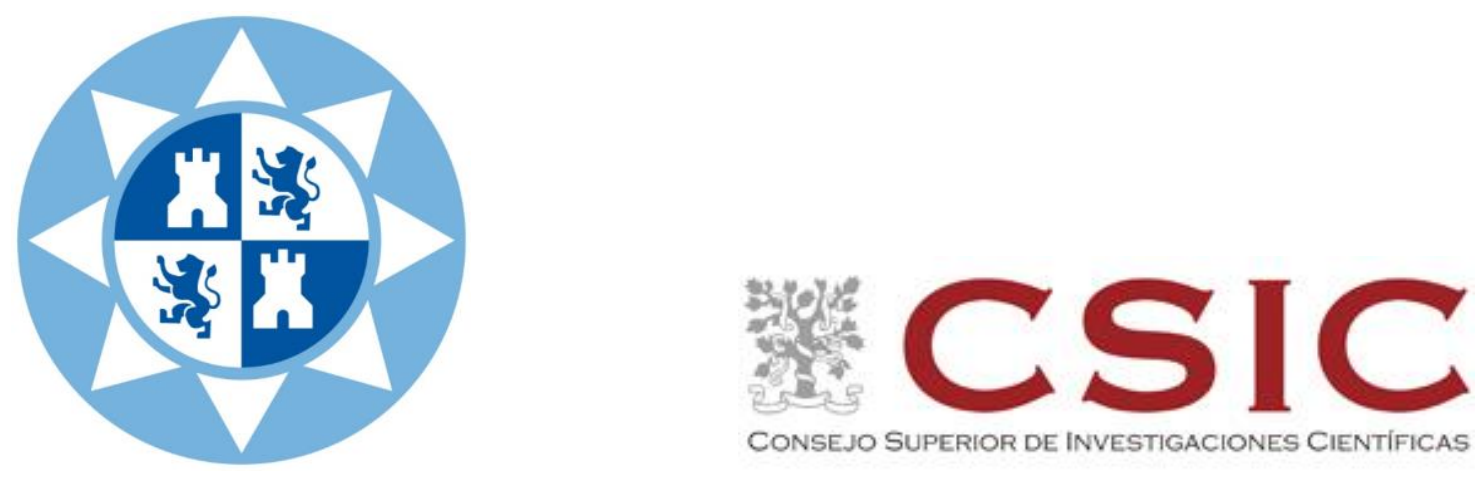

Universidad Politécnica de Cartagena

Departamento de Producción Vegetal

\title{
Programación de riego y déficit hídrico controlado en frutales de hueso \\ Tesis Doctoral
}

Francisco Pérez Sarmiento

Directores: Dr. Emilio Nicolás Nicolás

Dr. Oussama Mounzer

Tutor: Dr. Rafael Domingo Miguel

2015 


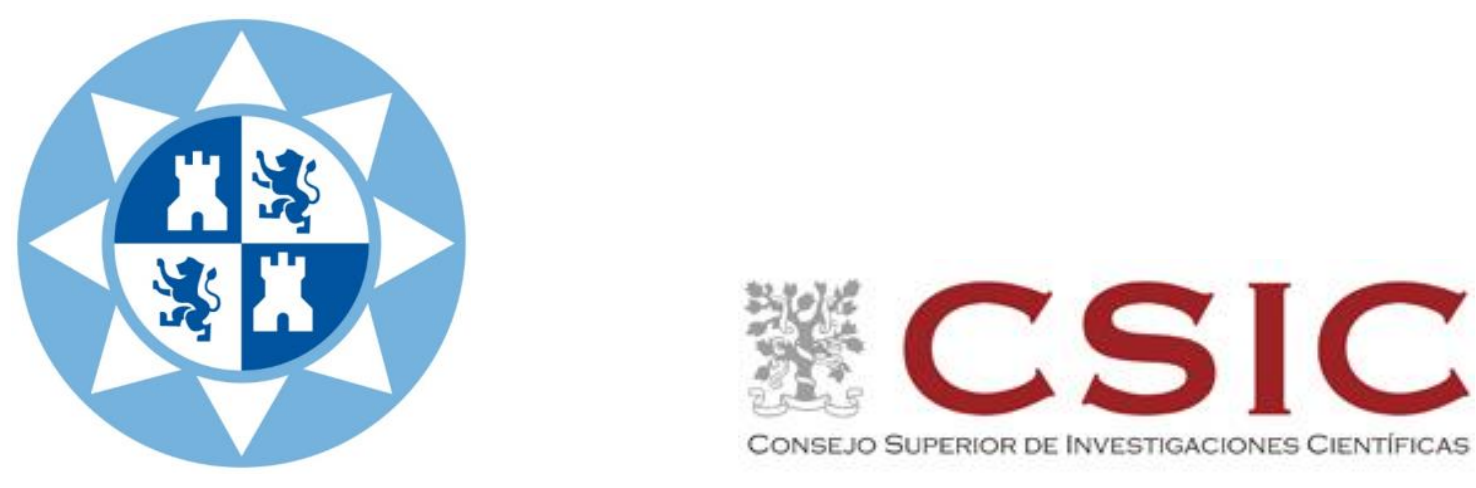

Technical University of Cartagena

Plant Production Department

\section{Irrigation scheduling and regulated deficit irrigation in stone fruit trees \\ Doctoral Thesis}

Francisco Pérez Sarmiento

Directors: Dr. Emilio Nicolás Nicolás

Dr. Oussama Mounzer

Tutor: Dr. Rafael Domingo Miguel

2015 



$$
\square
$$





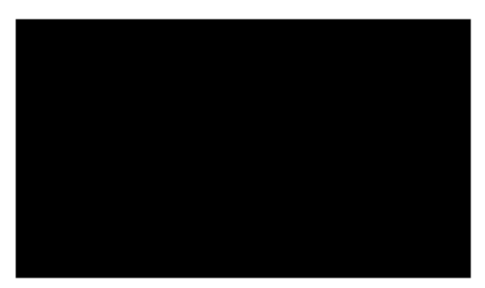





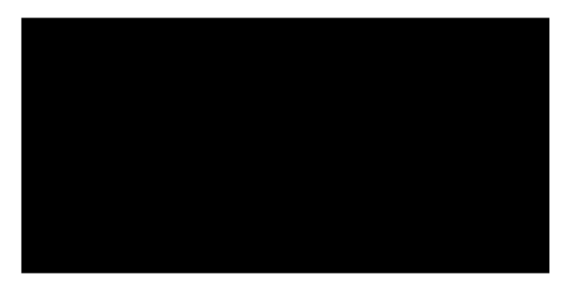






\section{Agradecimientos}

Llegados a este punto, es para mí un deber, agradecer a todos y cada uno de quienes han formado parte de mi vida durante el desarrollo de esta tesis doctoral.

En primer lugar a mis directores Dr. Emilio Nicolás Nicolás y Dr. Oussama Mounzer por sus enseñanzas, dedicación y permitirme llevar a cabo este capítulo de mi vida tan importante.

Al Dr. Rafael Domingo Miguel, por aceptar ser tutor de esta tesis, por su apoyo y disponibilidad.

Al Dr. Juan José Alarcón Cabañero por darme la oportunidad de formar parte de este equipo.

Al Dr. José Manuel Mirás Avalos por su gran ayuda para que este barco llegara a buen puerto.

A todos los miembros del departamento de riego del CEBAS por su acogimiento y hacerme sentir tan bien desde el primer día.

A mis compañeros de trabajo por esos buenos momentos pasados, esas risas echadas y también esos momentos de agobio entre medidas y evoluciones, con especial mención a la Dra. Rosalía Alcobendas Puig por su colaboración durante el desarrollo de esta tesis.

A quienes, de una forma u otra han formado parte de esta etapa de mi vida, gracias a todos.

Y por supuesto a mis padres y hermano, por su apoyo, comprensión y paciencia cuando el barco parecía no encontrar el puerto. 

MB Allajandra 



\section{ÍNDICE}

1. Introducción............................................................................................................................... 1

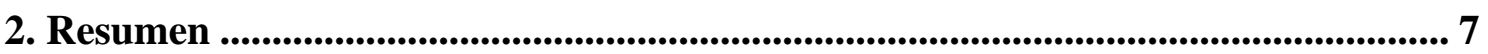

3. Objetivos.................................................................................................................................. 29

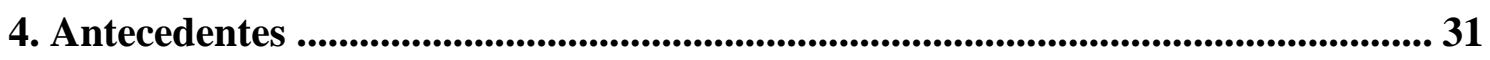

4.1. Situación actual de los frutales en el mundo, España y la Región de Murcia ..... 31

4.2. Riego de frutales: metodologías ......................................................................... 35

4.2.1. Métodos climatológicos ........................................................................... 36

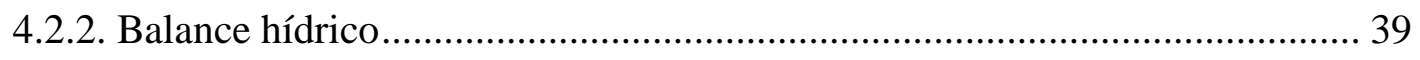

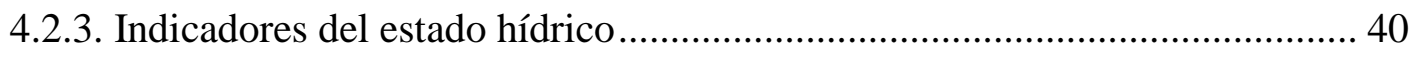

4.3. Concepto de Riego Deficitario Controlado (RDC) ………………..................... 50

4.4. Establecimiento de una estrategia de RDC ........................................................... 51

4.4.1. Periodos críticos del cultivo .................................................................... 52

4.4.2. Crecimiento vegetativo y de fruto ............................................................... 53

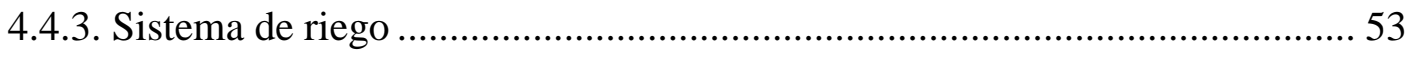

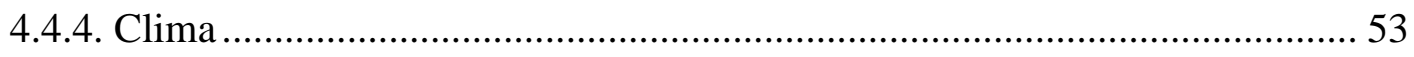

4.4.5. Características del suelo ............................................................................ 54

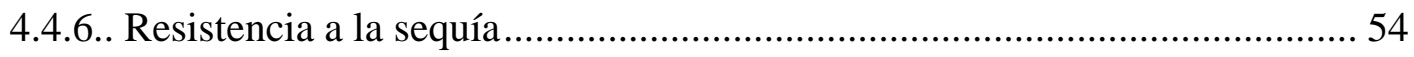

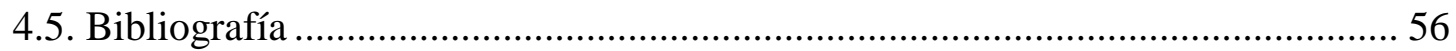

5. REFERENCE VALUES OF MAXIMUM DAILY TRUNK SHRINKAGE FOR IRRIGATION SCHEDULING IN MID-LATE MATURING PEACH TREES ... 63

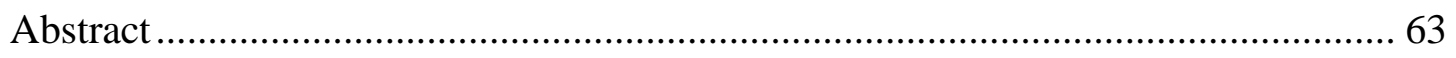

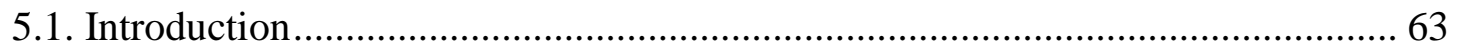

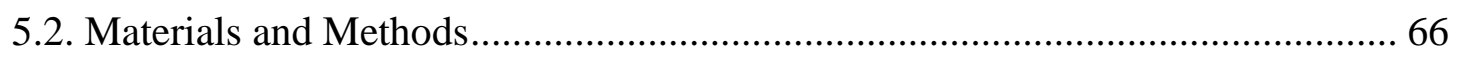

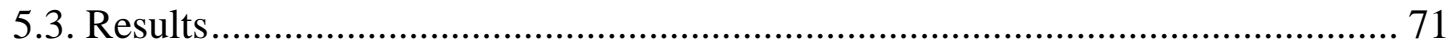

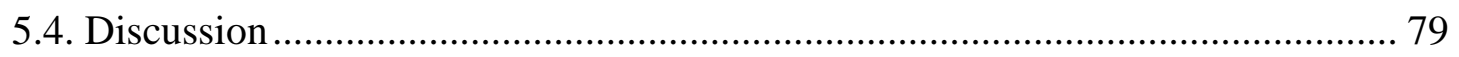

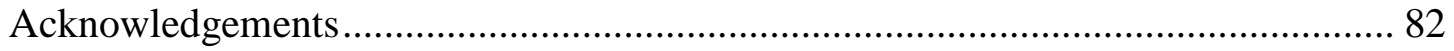

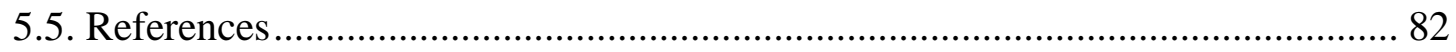




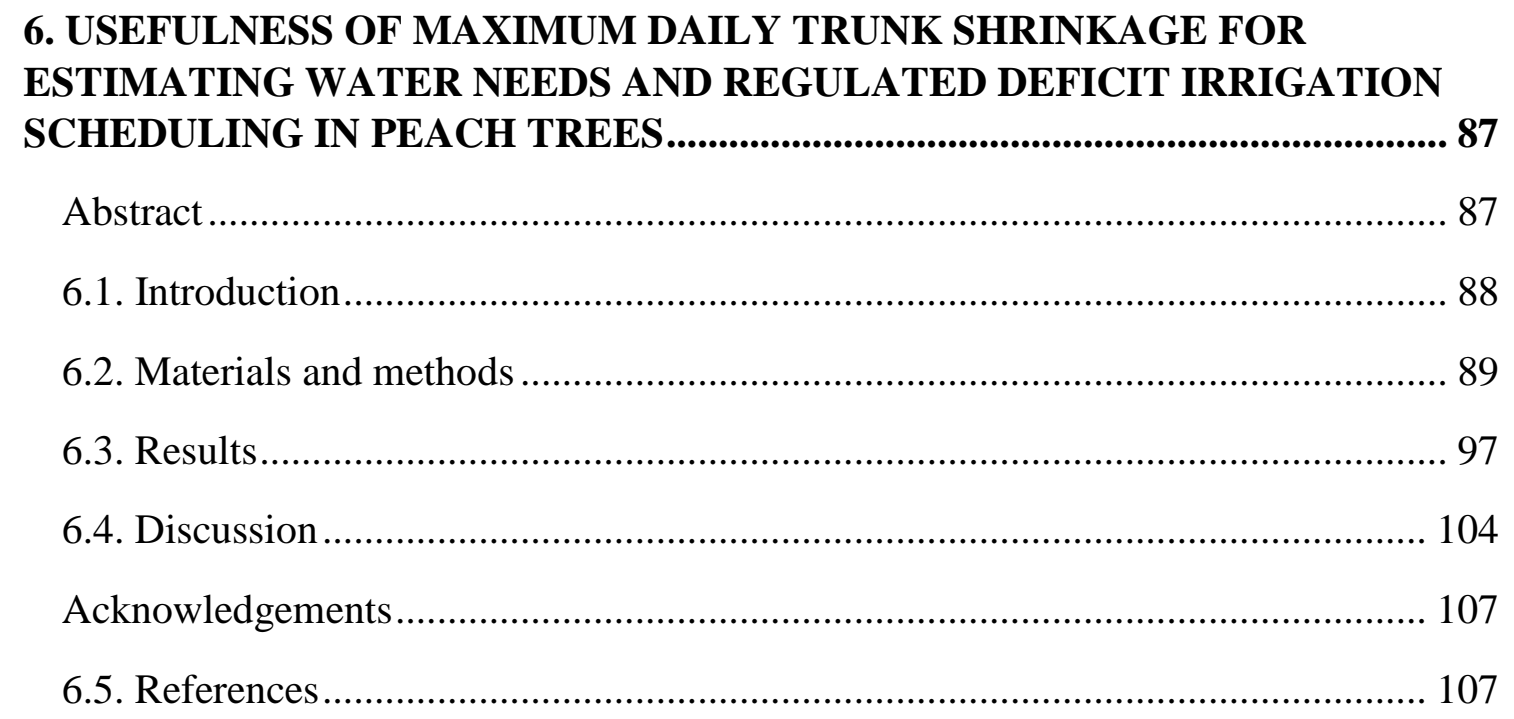

7. USE OF MIDDAY STEM WATER POTENTIAL FOR DEFICIT IRRIGATION SCHEDULING IN PEACH ORCHARDS IN SOUTH-EAST

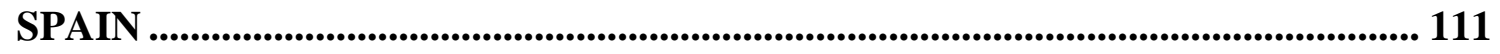

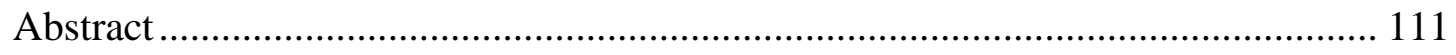

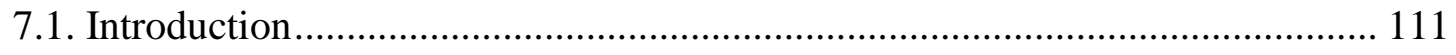

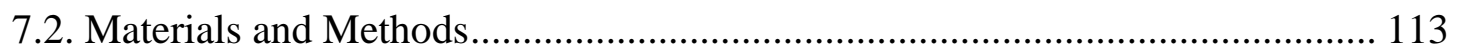

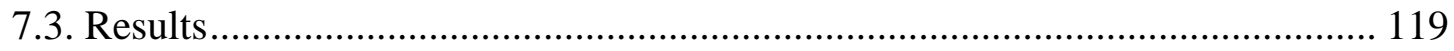

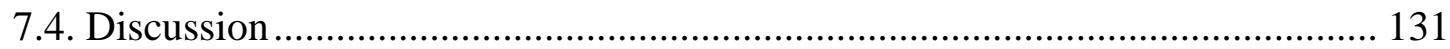

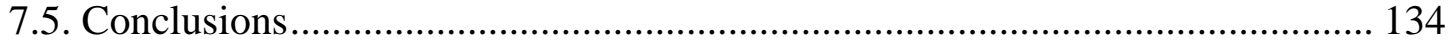

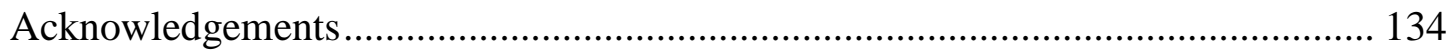

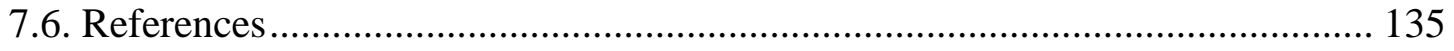

8. EFFECTS OF REGULATED DEFICIT IRRIGATION ON PHYSIOLOGY, YIELD AND FRUIT QUALITY IN APRICOT TREES................................... 139

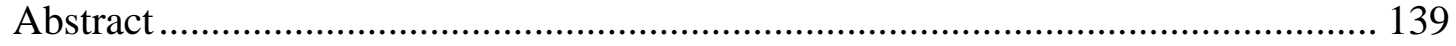

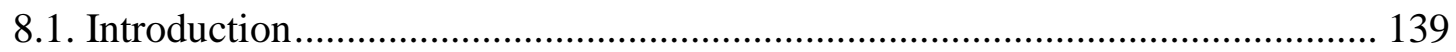

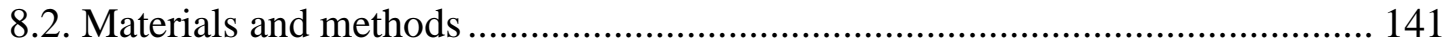

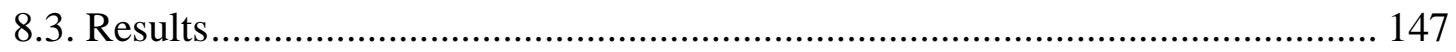

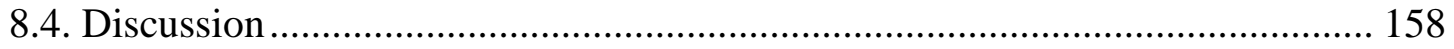

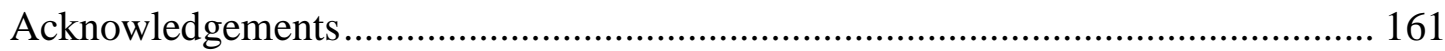

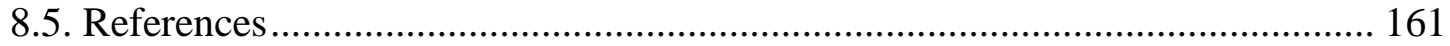

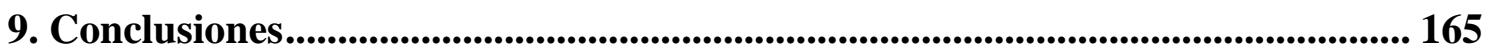

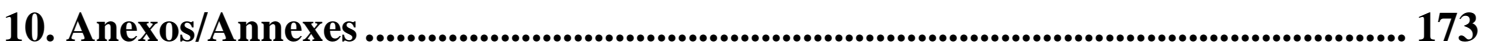




\section{Índice de Figuras}

Figura 4.1. Principales Comunidades Autónomas españolas en superficie y producción de melocotones (Datos extraidos de MAGRAMA 2015b). ..........................32

Figura 4.2. Principales Comunidades Autónomas españolas en superficie y producción de albaricoque (Datos extraidos de MAGRAMA 2015b).............................34

Figura 4.3. Parámetros derivados de las fluctuaciones diarias del diámetro del tronco: máximo diámetro diario de tronco (MXDT) y mínimo (MNDT), máxima contracción diaria (MCD) y la tasa de crecimiento del tronco (TCT) durante 3 días consecutivos.

Figure 5.1. Evolution of different climate parameters over the three years studied (2008-2010). Potential evapotranspiration $\left(\mathrm{ET}_{0}\right)$, mean daily temperature $\left(\mathrm{T}_{\mathrm{m}}\right)$, midday temperature $\left(\mathrm{T}_{\mathrm{mid}}\right)$, mean air vapour pressure deficit $\left(\mathrm{VPD}_{\mathrm{m}}\right)$ and midday air vapour pressure deficit $\left(\mathrm{VPD}_{\mathrm{mid}}\right)$.

Figure 5.2. Midday stem water potential $\left(\Psi_{\mathrm{s}}\right)$ and maximum daily trunk diameter shrinkage (MDS) dynamics over the three growing seasons studied (2008-2010). The relationships between both parameters are depicted for each season. FG and PH indicate fruit-growth and post-harvest stages, respectively.

Figure 5.3. Relationships of MDS with the maximum daily temperature $\left(\mathrm{T}_{\max }\right)$, midday temperature $\left(\mathrm{T}_{\mathrm{mid}}\right)$, maximum and midday air vapour pressure deficit (VPD ${ }_{\max }$ and $\mathrm{VPD}_{\text {mid }}$, respectively), pooling data for the three seasons studied (20082010). The regression equations for these relationships are presented in Tables 5.2 and 5.3. FG and PH indicate fruit-growth and post-harvest stages, respectively.

Figure 5.4. Relationship of MDS with potential evapotranspiration $\left(\mathrm{ET}_{0}\right)$ pooling data from the three consecutive seasons studied (2008-2010). The regression equations obtained for each season and the total pool of data are presented in Tables 5.2 and 5.3. FG and PH indicate fruit-growth and post-harvest stages, respectively......75

Figure 5.5. Relationship of MDS and midday stem water potential $\left(\Psi_{\mathrm{s}}\right)$ pooling data from the three consecutive seasons studied (2008-2010). The regression equations obtained for each season and the total pool of data are presented in Table 5.4. FG and $\mathrm{PH}$ indicate fruit-growth and post-harvest stages, respectively.

Figure 6.1. Precision ( $\mathrm{C}_{\mathrm{MDS}}$ ) and Regulated Deficit Irrigation (RDI) strategies based on threshold values for maximum daily shrinkage signal intensity (MDS SI) applied in stage II (S II) of fruit growth and post-harvest during the three years of the experiment.

Figure 6.2. Reference evapotranspiration $\left(\mathrm{ET}_{0}, \mathrm{~mm}\right)$, rainfall $(\mathrm{mm})$ and vapour pressure deficit (VPD, KPa) in Fuente Librilla. Monthly values from data collected during 2008, 2009 and 2010. 
Figure 6.3. Seasonal patterns of (A) cumulative applied water and (B) midday stem water potential values for each irrigation treatment for the 3 years of the experiment: $\mathrm{C}_{\mathrm{ETc}}$ (closed circles), $\mathrm{C}_{\mathrm{MDS}}$ (open squares) and RDI (open triangles) plants. Each data point is the mean of sixteen values. Asterisks indicate significant differences among treatments $(p<0.05)$. The interval between vertical lines, from left to right, represents the beginning of stages II and III of fruit growth and postharvest.

Figure 6.4. Maximum daily shrinkage $(\mathrm{A}, \mu \mathrm{m})$ evolution in the $\mathrm{C}_{\mathrm{ETc}}$ (closed circles), $\mathrm{C}_{\text {MDS }}$ (open squares) and RDI (open triangles) treatments, and maximum daily shrinkage signal intensity (MDS SI) (B) as $\mathrm{C}_{\mathrm{MDS}} / \mathrm{C}_{\mathrm{ETc}}$ ratio (open squares) and RDI/ $\mathrm{C}_{\mathrm{ETc}}$ ratio (open triangles) for the irrigation treatments during the fruit growth period 2008-2010. The interval between vertical short dash lines from left to right represent the beginning of stages II and III of fruit growth and post-harvest. Horizontal short dash lines represent the threshold values for MDS SI. Each data point is the mean of four values.

Figure 6.5. Water stress integral for each irrigation treatment, $\mathrm{C}_{\mathrm{ETc}}$ (black bars), $\mathrm{C}_{\mathrm{MDS}}$ (gray bars) and RDI (white bars) in the 2008, 2009 and 2010 growing seasons. Different letters on top of bars indicate significant differences according to Tukey's multiple range test $(p<0.05)$.

Figure 6.6. Pruning weight as a function of the irrigation treatment, $\mathrm{C}_{\mathrm{ETc}}$ (black bars), $\mathrm{C}_{\mathrm{MDS}}$ (gray bars) and RDI (white bars) in the 2008, 2009 and 2010 growing seasons. Each bar corresponds to the mean of sixteen trees. Different letters on top of bars indicate significant differences according to Tukey's multiple range test $(p<$ $0.05)$.

Figure 6.7. Percentage of fruit categories for each irrigation treatment, $\mathrm{C}_{\mathrm{ETc}}$ (black bars), $\mathrm{C}_{\mathrm{MDS}}$ (gray bars) and RDI (white bars) in the 2008, 2009 and 2010 growing seasons. Each bar corresponds to the mean of sixteen trees. Different letters on top of bars indicate significant differences according to Tukey's multiple range test $(p<$ $0.05)$.

Figure 7.1. Regulated deficit irrigation (RDI I: moderate deficit, RDI II: severe deficit) strategies based on threshold values for midday stem water potential $\left(\Psi_{\mathrm{s}}\right)$, applied in stage II (S II) of fruit growth and postharvest during the three years of the experiment.

Figure 7.2. Reference evapotranspiration $\left(\mathrm{ET}_{0}\right)$, rainfall $(\mathrm{mm})$ and VPD $(\mathrm{KPa})$ in Fuente Librilla. Monthly values from data collected during 2008, 2009 and 2010.....120

Figure 7.3. Volumetric soil water content down to $1 \mathrm{~m}$ depth $\left(\theta_{\mathrm{v}}\right)$, in $\mathrm{C}$ (closed circles), RDI I (open squares) and RDI II (open triangles) treatments for the three growing seasons studied (2008-2010). Each data point is the mean of three values. Asterisks indicate significant differences among treatments $(p<0.05)$. The interval between vertical lines, from left to right, represents the beginning of stages II and III of fruit growth and postharvest. Horizontal lines represent field capacity.....

Figure 7.4. Seasonal patterns of (A) cumulative applied water and (B) midday stem water potential values for each irrigation treatment for the 3 years of the experiment: C (closed circles), RDI I (open squares) and RDI II (open triangles) plants. Each data point is the mean of sixteen values. Asterisks indicate significant differences among 
treatments $(p<0.05)$. The interval between vertical lines, from left to right, represents the beginning of stages II and III of fruit growth and postharvest. Horizontal lines represent threshold values in each phenological stage for both RDI treatments.

Figure 7.5. Stress integral for each irrigation treatment, control (black bars), RDI I (gray bars) and RDI II (white bars) in the 2008, 2009 and 2010 growing cycles. Different letters on top of bars indicate significant differences according to Tukey's multiple range test $(p<0.05)$.

Figure 7.6. Net photosynthesis $\left(P_{n}, A\right)$ and stomatal conductance $\left(g_{s}, B\right)$ values averaged for the three studied growing seasons in $\mathrm{C}$ (closed circles), RDI I (open squares) and RDI II (open triangles) plants. Each data point is the mean of sixteen values. Asterisks indicate significant differences among treatments $(p<0.05)$. The interval between vertical lines, from left to right, represents the beginning of stages II and III of fruit growth and postharvest.

Figure 7.7. Pruning weight as a function of the irrigation treatment, control (black bars), RDI I (gray bars) and RDI II (white bars) in the 2008, 2009 and 2010 growing cycles. Each bar corresponds to the mean of sixteen trees. Different letters on top of bars indicate significant differences according to Tukey's multiple range test $(p<$ $0.05)$.

Figure 7.8. Relation between pruning weight and water applied in $\mathrm{C}$ (closed triangles), RDI I (open circles) and RDI II (closed circles) plants for the three studied growing seasons. Solid line represents the linear regression between pruning weight and water applied. Asterisks indicate a significant relationship between variables $(p<$ $0.01)$.

Figure 7.9. Shoot growth $(\mathrm{A}, \mathrm{cm})$ and fruit diameter $(\mathrm{B}, \mathrm{mm})$ evolution in $\mathrm{C}$ (closed circles), RDI I (open squares) and RDI II (open triangles) tress for the three studied growing seasons. The interval between vertical lines, from left to right, represents the beginning of stages II and III of fruit growth and postharvest. Each value is the mean of 160 measurements.

Figure 7.10. Percentage of fruit categories for each irrigation treatment, control (black bars), RDI I (gray bars) and RDI II (white bars) in the 2008, 2009 and 2010 growing cycles. Each bar corresponds to the mean of sixteen trees. Different letters on top of bars indicate significant differences according to Tukey's multiple range test $(p<0.05)$......

Figure 8.1. Percentages of crop evapotranspiration $\left(\mathrm{ET}_{\mathrm{c}}\right)$ applied to the regulated deficit irrigation (RDI) treatment for each phenological stage of "Búlida" apricot trees over the experimental period.

Figure 8.2. Reference evapotranspiration $\left(\mathrm{ET}_{0}\right)$, rainfall $(\mathrm{mm})$ and VPD $(\mathrm{KPa})$ at the experimental site. Monthly values from data collected during 2008, 2009 and 2010...144

Figure 8.3. Volumetric soil water content down to $1 \mathrm{~m}$ depth $\left(\theta_{\mathrm{v}}\right)$, in $\mathrm{C}$ (closed circles) and RDI (open circles) treatments for the three growing seasons studied (2008-2010). Each data point is the mean of four values. Asterisks indicate significant differences between treatments $(p<0.05)$. The intervals between vertical 
lines from left to right represent the beginning of the different phenological stages for apricot trees during the experimental period. Horizontal lines represent soil field capacity.

Figure 8.4. Midday leaf water potential in $\mathrm{C}$ (closed circles) and RDI (open circles) trees for the three studied growing seasons. Each data point is the mean of sixteen values. The intervals between vertical lines from left to right represent the beginning of the different phenological stages for apricot trees during the experimental period. Asterisks indicate significant differences between treatments $(p<0.05)$.

Figure 8.5. Midday stem water potential in C (closed circles) and RDI (open circles) trees for the three studied growing seasons. Each data point is the mean of sixteen values. The intervals between vertical lines from left to right represent the beginning of the different phenological stages for apricot trees during the experimental period. Asterisks indicate significant differences between treatments $(p<0.05)$.

Figure 8.6. Stress integral values for each irrigation treatment, control (black bars) and RDI (white bars) in the 2008, 2009 and 2010 growing seasons. Asterisks indicate significant differences between treatments $(p<0.05) \ldots$

Figure 8.7. Net photosynthesis $\left(\mathrm{P}_{\mathrm{n}}, \mathrm{A}\right)$ and stomatal conductance $\left(\mathrm{g}_{\mathrm{s}}, \mathrm{B}\right)$ values averaged for the three studied growing seasons in $\mathrm{C}$ (closed circles) and RDI (open circles) trees. Each data point is the mean of sixteen values. Asterisks indicate significant differences between treatments $(p<0.05)$. The intervals between vertical lines from left to right represent the beginning of the different phenological stages for apricot trees during the experimental period.

Figure 8.8. Fruit diameter $(\mathrm{mm})$ evolution in $\mathrm{C}$ (closed circles) and RDI (open circles) trees for the three studied growing seasons. Each data point is the mean of 200 values. The intervals between vertical lines from left to right represent the beginning of the different phenological stages for apricot trees during the experimental period. 


\section{Índice de Tablas}

Table 5.1. Characteristics of the studied trees over the three growing seasons studied (2008-2010). TCSA indicates trunk cross-sectional area.

Table 5.2. Parameters of the reference equations for maximum daily trunk shrinkage (MDS) as a function of selected environmental variables during the three seasons studied (2008-2010). ANCOVA results for the comparison of the slopes and intercepts of the fitted curves among the seasons studied.

Table 5.3. Parameters of the reference equations for maximum daily trunk shrinkage (MDS) as a function of selected environmental variables during the fruit-growth and post-harvest stages of the three seasons studied (2008-2010).

Table 5.4. Parameters of the reference equations for maximum daily trunk shrinkage (MDS) as a function of midday stem water potential $\left(\Psi_{\mathrm{s}}\right)$ for the whole season and during the fruit-growth and post-harvest stages of the three years studied (20082010). ANCOVA results for the comparison of the slopes and intercepts of the fitted curves among the seasons studied.

Table 6.1. Average volumetric soil water content $\left(\mathrm{m}^{3} \mathrm{~m}^{-3}\right)$ for the three irrigation treatments considered at different phenological periods for the three studied growing seasons. Each data is the mean of all values in the corresponding stage. Different letters in each column indicate significant differences among treatments according to Tukey's multiple range test $(p<0.05)$.

Table 6.2. Average maximum daily shrinkage signal intensity (MDS SI) in different stages of fruit growth for the three studied growing seasons. Each data is the mean of all values in the corresponding stage. Different letters next to a value in each year indicate significant differences according to Tukey's multiple range test $(p<0.05) \ldots 100$

Table 6.3. Crop load, yield, water productivity (WP) and water spends/savings in each non-critical period and total in response to the different irrigation treatments for the experimental period 2008-2010. Different letters in the column within each year indicate significant differences among treatments according to Tukey's multiple range test $(p<0.05)$.

Table 6.4. Fruit firmness, $\mathrm{pH}$, soluble solids content (SSC), tritratable acidity (TA) and SSC/TA ratio at harvest for all treatments. Values are the mean of 100 measurements. Different letters in each column within each year indicate significant differences according to Tukey's multiple range test $(p<0.05)$.

Table 7.1. Average midday stem water potential in different stages of fruit growth for the three studied growing seasons. Each data is the mean of all values in the corresponding stage. Different letters next to a value in each year indicate significant differences among treatments according to Tukey's multiple range test $(p<0.05) \ldots . .123$

Table 7.2. Stress integral average values (MPa days) in different stages during the three seasons of the experiment. These values were calculated with data of the midday stem water potential of Figure 7.4B. Each data point is the mean of all values 
in the corresponding stage. Different letters next to a value in each year indicate significant differences according to Tukey's multiple range test $(p<0.05)$.

Table 7.3. Flower density, fruit set and trunk cross sectional area (TCSA) average values in different treatments during the three seasons of the experiment. Each data point is the mean of 160 values per treatment (Fruit set and flower density) and 64 values per treatment (TCSA). Different letters next to a value in each year indicate significant differences according to Tukey's multiple range test $(p<0.05)$.

Table 7.4. Crop load (number of fruits tre $\mathrm{e}^{-1}$ ), yield $\left(\mathrm{kg} \mathrm{tree}^{-1}\right)$, water productivity (WP, $\mathrm{kg} \mathrm{m}^{-3}$ ) and water spends/savings in each non-critical period and total in response to the irrigation treatments for the experimental period 2008-2010. Different letters next to a value in each year indicate significant differences according to Tukey's multiple range test $(p<0.05)$.

Table 7.5. Fruit firmness $(\mathrm{N}), \mathrm{pH}$, soluble solids content (SSC, ${ }^{\circ}$ Brix), tritratable acidity (TA, g $100 \mathrm{~mL}^{-1}$ ) and SSC/TA ratio at harvest for all treatments. Values are the mean of 100 measurements. Different letters next to a value in each year indicate significant differences according to Tukey's multiple range test $(p<0.05)$.

Table 8.1. Cumulative applied water $(\mathrm{mm})$ during the different stages of fruit growth for the three studied growing seasons, in $\mathrm{C}$ and RDI trees.

Table 8.2. Average values of midday stem water potential $\left(\Psi_{\mathrm{s}}, \mathrm{MPa}\right)$, leaf water potential $\left(\Psi_{1}, \mathrm{MPa}\right)$, net photosynthesis $\left(\mathrm{P}_{\mathrm{n}}, \mu \mathrm{mol} \mathrm{m} \mathrm{m}^{-2} \mathrm{~s}^{-1}\right)$ and stomatal conductance

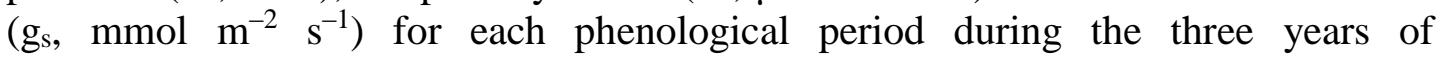
experimental period, in the control (C) and regulated deficit irrigation (RDI) treatments.

Table 8.3. Fruit set $(\%)$, trunk cross sectional area (TCSA, $\left.\mathrm{cm}^{2}\right)$, yield $\left(\mathrm{kg} \mathrm{tree}^{-1}\right)$, water productivity (WP, $\mathrm{kg} \mathrm{m}^{-3}$ ) and water savings as a function of the irrigation treatments for the experimental period 2008-2010.

Table 8.4. Fruit firmness $(\mathrm{N}), \mathrm{pH}$, soluble solids content (SSC, ${ }^{\circ}$ Brix), tritratable acidity (TA, g $100 \mathrm{~mL}^{-1}$ ) and SSC/TA ratio at harvest as a function of the treatment for the three experimental seasons.

Table 8.5. Skin and flesh fruit colour values (reflectance measurements $L^{*}, H^{o}, C^{*}$ ) at harvest in the control (C) and regulated deficit irrigation (RDI) treatments, for the three experimental seasons. 


\section{Índice de Fotografías}

Fotografía 4.1. Melocotonero "Catherine” en producción (Fuente propia)....................33

Fotografía 4.2. Albaricoquero "Búlida" en producción (Fuente propia). .......................34

Fotografía 4.3. Hoja tapada y medición con cámara de presión. Soil Moisture Equip.

Corp, model 3000, Santa Barbara, CA, USA (Fuente propia).

Fotografía 4.4. Sistema portátil para medida de fotosíntesis LI-6400, Li-Cor, Lincoln, NE, USA (Fuente propia).

Fotografía 4.5. Equipo Time Domain Reflectometry (TDR). Model 1502C,

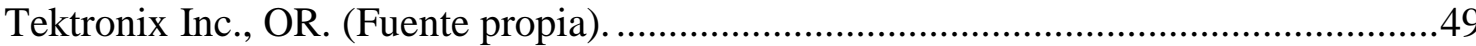

Fotografía 4.6. Sonda de neutrones. Model 4300, Troxler Electronic Laboratories

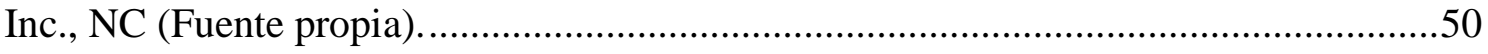

Picture 5.1. View of the experimental site (Own source) .............................................66

Picture 5.2. Automatic weather station placed within the experimental orchard (Own source). .68

Picture 5.3. Harvested "Catherine" peaches (Own source)........................................69

Picture 5.4. Measurement of the tree canopy (Own source) ....................................... 70

Picture 6.1. Plot location. (Source Google Maps). .....................................................90

Picture 6.2. LVDT sensor installed in peach "Catherine" (Own source) .......................94

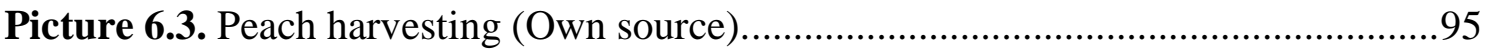

Picture 7.1. Pressure chamber (Own source). .........................................................116

Picture 7.2. Fruit Calibrator (Own Source) ................................................................ 117

Picture 7.3. Durofel penetrometer (Own Source)...................................................118

Picture 8.1. Plot location. (Source: Google Maps)...................................................141

Picture 8.2. Recently harvested apricots (Own Source)............................................146

Picture 8.3. Minolta chromameter (Own Source). ...................................................146 


\section{INTRODUCCIÓN/INTRODUCTION}





\section{Introducción}

La actual y creciente presión sobre los recursos hídricos causada por la competencia por el agua entre agricultura, industria y población, crea una necesidad urgente de aumentar la productividad del agua de riego y desarrollando nuevas herramientas y métodos de ahorro de agua en la agricultura. Por tanto, resulta necesario el desarrollo de nuevas estrategias para optimizar el agua.

Una de las técnicas más prometedoras para lograr este objetivo es el Riego Deficitario Controlado (RDC), cuyo concepto es controlar el crecimiento vegetativo del cultivo sin afectar a su producción posibilitando incluso obtener otras ventajas, como la mejora de la calidad del fruto. En este contexto, es de vital importancia determinar el estado hídrico de la planta con el fin de detectar rápidamente cualquier estrés hídrico y, de esta manera, poder gestionar de forma adecuada el riego durante el desarrollo del cultivo. Para lograrlo, se necesitan herramientas precisas que determinen el estado hídrico de la planta. En este sentido, el uso de indicadores del estado hídrico basados en medidas en planta se ha convertido en una buena herramienta para la planificación del riego de precisión, ya que controla muchos procesos fisiológicos y la productividad de los cultivos.

El método más utilizado para evaluar el estado hídrico de los árboles para la programación del riego es el potencial hídrico de tallo al mediodía $\left(\Psi_{\mathrm{t}}\right)$. Sin embargo, la principal desventaja de $\Psi_{\mathrm{t}}$ es que no se puede automatizar y, por lo tanto, son necesarios viajes frecuentes al campo y mano de obra. Por otro lado, el uso de las fluctuaciones diámetro del tronco ha demostrado también su utilidad como indicadores del estado hídrico de la planta ya que, entre otras ventajas, permiten registros continuos y automatizados con la consiguiente reducción de la mano de obra necesaria para su medida.

El melocotonero [Prunus persica (L.) Batsch] es uno de los cultivos frutales más extendidos en todo el mundo, siendo la segunda especie frutal en producción después del manzano. Su gran aceptación por parte de los consumidores se debe a su gran variedad de usos, incluyendo el consumo en fresco. Su principal país productor es China, mientras España ocupa el cuarto lugar. En la Región de Murcia, el melocotonero es el primer frutal de hueso en rendimiento y el segundo en superficie cultivada tras el almendro. La Región de Murcia, con una superficie cultivada de 10.713 hectáreas y una 
producción de 171.526 t ocupa el tercer lugar en la producción de melocotón en el país, tras Aragón y Cataluña (MAGRAMA, 2015).

El albaricoquero (Prunus armeniaca L.) es una de las especies frutales más importantes a nivel mundial ya que su fruto es muy apreciado por los consumidores (Roussos et al., 2011). La producción mundial de albaricoque es de, aproximadamente, 2,5 millones de toneladas por año siendo Turquía el país productor líder, según la Organización para la Agricultura y la Alimentación, con aproximadamente 676.000 t (FAO, 2013). España produce alrededor de 132.000 t de una zona de cultivo de 20.000 hectáreas. En la Región de Murcia (SE España) casi toda la superficie cultivada $(\approx 10,000$ ha en 2014$)$ se encuentra bajo regadío. Esta zona de cultivo produjo $79.000 \mathrm{t}$ en 2014 (60\% de la producción de albaricoque en España) (MAGRAMA, 2015). El cultivar más importante de albaricoquero es el 'Búlida', el cual representa el 66\% de la producción total en la Región de Murcia (CARM, 2009).

En el presente estudio, se tomaron medidas de potencial hídrico del tallo al mediodía $\left(\Psi_{\mathrm{t}}\right)$ y máxima contracción diaria del tronco $(\mathrm{MCD})$ durante un período de 3 años en melocotonero [Prunus persica (L.) Batsch cv. 'Catherine'] y albaricoquero [Prunus armeniaca L. cv. 'Búlida'] con el fin de evaluar el uso de estos indicadores del estado hídrico basados en la planta para la programación del Riego Deficitario Controlado (RDC).

Esta tesis doctoral se estructuró en 10 capítulos, de la siguiente forma:

En primer lugar (capítulo 2), se presenta un resumen de los contenidos de esta tesis incluyendo los objetivos, los materiales y métodos usados y los resultados obtenidos. En el apartado siguiente (capítulo 3) se enumeran los objetivos de esta investigación. Seguidamente, se expone brevemente el estado actual de conocimientos sobre el tema (capítulo 4).

Los capítulos 5, 6, 7 y 8 describen las cuatro experiencias que se han llevado a cabo, divididos en un breve resumen, introducción, materiales y métodos utilizados, resultados, discusión, conclusiones (en algunos casos), agradecimientos y bibliografía consultada. 
En el capítulo 9 se recogen las principales conclusiones derivadas de este trabajo de investigación, incluyendo tanto unas generales como parciales. En el capítulo 10 (anexos), se adjunta como indicador de calidad de esta Tesis el artículo "Effects of regulated deficit irrigation on physiology and fruit quality in apricot trees", publicado en la revista Spanish Journal of Agricultural Research 2010 Vol 8 (S2) S86S94 ISSN 1695-971-X, revista perteneciente al JCR y encuadrada en el Q2 dentro del área científica 'Agriculture Multidisciplinary'. 


\section{Introduction}

The current increasing pressure on water resources caused by the competition for water between agriculture, industry and population, creates an urgent need to increase irrigation water productivity and to develop and provide new tools and water conservation methods in agriculture. This requires the development of new strategies to optimize water.

One of the most promising techniques to achieve this objective is Regulated Deficit Irrigation (RDI), whose concept was to control excessive vegetative growth and enhance fruit growth, besides other advantages such as improvements of fruit quality and taste. In this context, it is of paramount importance to determine plant water status in order to rapidly detect any water stress and correctly manage irrigation over the growing season. To achieve this, precise tools for assessing plant water status are needed; in this sense, the use of plant-based water status indicators has become very popular for planning precise irrigation, because it controls many physiological processes and crop productivity.

The most widely used approach for evaluating tree water status for irrigation scheduling is the midday stem water potential $\left(\Psi_{\mathrm{s}}\right)$. However, the main disadvantage of $\Psi_{\mathrm{s}}$ is that it cannot be automated and thus frequent trips to the field and manpower are needed. The use of trunk diameter fluctuations has renewed the interest in usingthese measurements as a plant water status indicator. This technique permits continuous and automated registers with a reduction in the labour needed for the measurement procedure.

Peach [Prunus persica (L.) Batsch] is one of the most extended fruit crops worldwide, being the second fruit species in production after apple. Its great acceptance by consumers is due to its variety of uses, including fresh consumption. Its main producing country is China, whereas Spain ranks fourth. In the Murcia Region, peach is the first stone fruit in yield and the second one in cultivated surface after almonds. Murcia region, with a cultivated area of 10,713 ha and a production of 171,526 $\mathrm{t}$ ranks third in peach production within the country, after Aragon and Catalonia (MAGRAMA, 2015). 
Apricot (Prunus armeniaca L.) is one of the most important fruit species worldwide since its fruit is highly appreciated by consumers (Roussos et al., 2011). Apricot world production is about 2.5 million t per year and Turkey is the leading producing country, according to the Food and Agriculture Organization, with approximately 676,000 $\mathrm{t}$ (FAO, 2013). Spain produces about 132,000 t from a cultivation area of 20,000 ha. In the Murcia Region (SE Spain) almost all the cultivated area $(\approx 10,000$ ha in 2014$)$ is irrigated. This cultivation area yielded $79,000 \mathrm{t}$ in 2014 (60\% of apricot production in Spain) (MAGRAMA, 2015). The most important Spanish apricot cultivar is "Búlida", which represents $66 \%$ of the total production in the Murcia Region (CARM, 2009).

In the current study, measurements of midday stem water potential $\left(\Psi_{\mathrm{s}}\right)$ and maximum daily trunk shrinkage (MDS) were taken over a 3-year period in peach trees [Prunus persica (L.) Batsch cv. 'Catherine'] and in apricot trees [Prunus armeniaca L. cv. 'Búlida'] in order to assess the use of these plant-based indicators for regulated deficit irrigation (RDI) scheduling.

This doctoral thesis was structured in 10 chapters, as follows:

First (chapter 2), a summary of the contents of this thesis is presented including the aims, the materials and methods used and the results obtained. In the next section (Chapter 3), the objectives of this research are enumerated. Afterwards, the current state of the art on the subject is briefly described (Chapter 4).

Chapters 5, 6, 7 and 8 described the four experiments that have been carried out. These chapters are clearly and concisely presented, with a brief summary, introduction, materials and methods, results, discussion, conclusions (in some cases), acknowledgments and references.

Chapter 9 collects the main conclusions from this thesis, including both general and partial conclusions. In chapter 10 (annexes), attached to this thesis as a quality indicator, the article entitled "Effects of regulated deficit irrigation on physiology and fruit quality in apricot trees" is presented. It was published in the Spanish Journal of Agricultural Research in 2010 Vol 8 (S2) S86-S94 ISSN 1695-971-X, journal included in the JCR and ranked within the $2^{\text {nd }}$ quartile in the 'Agriculture Multidisciplinary'category. 
RESUMEN/SUMMARY 



\section{Resumen}

La escasez y baja calidad de los recursos hídricos junto a la presión del desarrollo turístico, industrial y residencial hace que la agricultura en las regiones semiáridas afronte situaciones limitantes de modo casi permanente. Este sector consume más del $70 \%$ de los recursos hídricos disponibles ocasionando, en algunos casos, la sobreexplotación de los recursos hídricos subterráneos y la pérdida de calidad del agua. A pesar de ello, resulta difícil abordar esta problemática debido a varias razones entre las que se incluyen:

- Las consecuencias del uso excesivo del agua y fertilizantes en una agricultura intensiva no son tenidas en cuenta a medio y largo plazo.

- La gestión eficaz del recurso hídrico es una tarea colectiva ya que su implementación requiere la participación de todos los agentes implicados, tanto los gestores de los recursos hídricos como los agricultores o usuarios finales.

La poca sensibilización de los usuarios sobre las dimensiones del problema de escasez de agua y sus consecuencias, en algunos casos.

- $\quad$ La baja utilización de herramientas/técnicas que permitan ahorrar agua en el sector agrícola.

Para mejorar esta situación se requiere un esfuerzo colectivo entre quién gestiona los recursos hídricos y quién los utiliza. Los primeros deben buscar nuevas políticas de gestión y sistemas de gobernanza participativa mientras que los segundos deben cambiar sus prácticas de modo que consigan producciones más elevadas y de mejor calidad con una menor utilización de recursos hídricos.

Los agricultores, apoyados por los estudios de investigación y la introducción de nuevas tecnologías, pueden paliar las consecuencias de escasez de agua mediante varias opciones complementarias:

1) Riego de precisión: esta estrategia pretende minimizar las pérdidas de agua que suelen producirse durante los eventos de riego. Requiere de un sistema eficiente y una metodología de programación de riego que tome en cuenta las relaciones hídricas en el sistema suelo-planta-atmósfera. 
2) Riego deficitario: el hecho de que la respuesta de los cultivos al déficit hídrico es variable en función del estado fenológico de la planta permite, durante las fases fenológicas no críticas, reducir los aportes de riego por debajo de las necesidades hídricas máximas sin provocar mermas en la producción final.

3) Uso de aguas no convencionales: las aguas municipales regeneradas representan un volumen considerable del consumo total agrícola, por ello suelen producirse en las cercanías de zonas agrícolas. Además, contienen una alta concentración en minerales y materias orgánicas que podrían cubrir parte de las necesidades nutritivas de las plantas.

Por estos motivos, en los últimos años han cobrado gran importancia los estudios que abordan la aplicación de estrategias de riego deficitario controlado (RDC), las cuales además del ahorro hídrico que suponen, pueden incluso resultar en mejoras de algunos aspectos cualitativos de las cosechas obtenidas (Fereres y Soriano, 2007).

En base a ello, ha aumentado el interés por las medidas del estado hídrico basadas en la planta ya que ésta integra el efecto tanto de la disponibilidad de agua en el suelo, como de la demanda evaporativa y, por ello, proporcionan una información más adecuada para determinar el efecto de las restricciones de agua sobre la actividad fisiológica de la planta y para la programación del riego (Jones, 2004). Cabe diferenciar estos indicadores del estado hídrico en dos grupos, según permitan la obtención de datos de forma continua (máxima contracción diaria de tronco, $\mathrm{MCD}$ ), o no (potencial hídrico de tallo, $\Psi_{\mathrm{t}}$ ), a fin de poder automatizar el riego.

En el contexto mundial, el melocotonero [Prunus persica (L.) Batsch] es uno de los cultivos frutales más extendidos, siendo la segunda especie frutal en producción tras el manzano. Su gran aceptación por parte del consumidor se debe a su diversidad de usos, entre ellos su consumo en fresco. Su principal país productor es China, mientras que España ocupa la cuarta posición. En la Región de Murcia el melocotonero es el primer frutal de hueso en producción y el segundo en superficie de cultivo tras el almendro. La Región de Murcia, con una superficie cultivada de 10.713 ha y una producción de 171.526 t, ocupa el tercer lugar en producción de melocotón dentro del territorio nacional, tras Aragón y Cataluña (MAGRAMA, 2015). 
En el caso del albaricoquero (Prunus armeniaca L.) su producción mundial supera las 2,5 millones de toneladas, siendo Turquía el principal productor, seguido de Irán, mientras que España ocupa la 9 a posición (FAO, 2013). En España, la Región de Murcia, con aproximadamente un $60 \%$ de la producción nacional, ocupa el primer lugar, tanto en producción como en superficie cultivada, con una gran diferencia respecto al resto de Comunidades Autónomas (MAGRAMA, 2015).

Por todo ello, los objetivos generales de esta tesis fueron:

- Obtener ecuaciones de referencia a partir de medidas continuas de las fluctuaciones del diámetro del tronco con sensores en planta para su uso en la programación de riego en melocotoneros adultos [Prunus persica (L.) Batsch cv "Catherine"], calculando la máxima contracción diaria del tronco (MCD) y relacionándola con variables climáticas y el potencial hídrico de tallo.

- Cuantificar las necesidades hídricas de la planta en base a medidas de la MCD como herramienta de precisión en la programación de riego manteniendo los

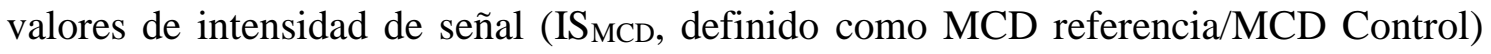
cercanos a la unidad durante toda la estación de crecimiento.

- Evaluar el uso de la MCD como indicador en planta para la programación del RDC, estableciendo para ello un umbral de la IS de 2 durante los períodos de crecimiento no críticos para esta estrategia de riego, evaluando para ello la producción y calidad de cosecha en melocotoneros adultos [Prunus persica (L.) Batsch cv "Catherine"].

- Evaluar el uso del potencial hídrico de tallo como indicador en planta para la programación del riego deficitario controlado (RDC), estableciendo para ello umbrales del mismo para melocotoneros adultos [Prunus persica (L.) Batsch cv "Catherine"].

- Evaluar el efecto del RDC en las relaciones hídricas de la planta, producción y calidad del fruto en albaricoqueros adultos [Prunus armeniaca (L.) cv. "Búlida"] durante tres años consecutivos.

Para alcanzar estos objetivos, se realizaron ensayos durante 3 años consecutivos en una finca comercial situada en el valle de Mula (Murcia) $\left(37^{\circ} 55^{\prime} \mathrm{N}, 1^{\circ} 25^{\prime} \mathrm{W}, 360 \mathrm{~m}\right.$ 
sobre el nivel del mar). La climatología fue típicamente mediterránea con inviernos suaves, veranos secos y calurosos y baja pluviometría (entre 188 y $304 \mathrm{~mm}$ anuales durante los 3 años de estudio). Se seleccionaron dos parcelas dentro de la misma finca. Una de ellas de 0,5 ha de melocotonero [Prunus persica (L.) Batsch] cv. "Catherine" (variedad de media-tardía estación), injertado sobre patrón híbrido de melocotonero $\mathrm{x}$ almendro GF-677, de 9 años de edad al inicio de la experiencia (2008) y con un marco de plantación de 4 × $6 \mathrm{~m}$. El suelo de la parcela es calcáreo, pedregoso, posee una textura franco-arenosa, baja materia orgánica y una capacidad de campo de $0,25 \mathrm{~m}^{3} \mathrm{~m}^{-3}$, aproximadamente.

Por otro lado, una parcela de 1 ha de albaricoquero [Prunus armeniaca L.] cv. “Búlida”, injertado sobre patrón franco de 'Real Fino', con una edad de 9 años al inicio de los experimentos y con un marco de plantación de 6 x $8 \mathrm{~m}$. El suelo de la parcela es altamente calcáreo, con una textura franco arcillosa, bajo en materia orgánica y una capacidad de campo de $0,31 \mathrm{~m}^{3} \mathrm{~m}^{-3}$, aproximadamente.

En ambos casos, el agua de riego procedía de un pozo cercano con valores de conductividad eléctrica en torno a $0,6 \mathrm{dS} \mathrm{m}^{-1}$.

Las medidas realizadas a lo largo del periodo experimental fueron:

- Los datos climáticos se registraron en una estación meteorológica automatizada situada en el huerto experimental.

- $\quad$ El contenido volumétrico de agua en el suelo $\left(\theta_{\mathrm{v}}, \mathrm{m}^{3} \mathrm{~m}^{-3}\right)$ se determinó mediante la técnica de la reflectometría en el dominio de tiempo (RDT) (Tektronix device, Model 1502C, Tektronix Inc., OR) de acuerdo con Moreno et al. (1996) y con una sonda de neutrones (Model 4300, Troxler Electronic Laboratories Inc., NC), tomando las medidas por la mañana, cada 7 a 15 días, durante el período experimental.

El potencial hídrico de tallo al mediodía $\left(\Psi_{\mathrm{t}}, \mathrm{MPa}\right)$ y el potencial hídrico foliar $\left(\Psi_{\mathrm{h}}, \mathrm{MPa}\right)$ se midieron usando una cámara de presión tipo Scholander (Soil Moisture Equip. Corp., model 3000, Santa Barbara, CA, USA) y siguiendo los procedimientos descritos por Turner (1981).

Las fluctuaciones micrométricas del diámetro del tronco (FDT) se registraron durante el periodo experimental con la ayuda de transductores de 
desplazamiento linear variable (sensores TDLV, Solartron Metrology, Bognor Regis, $\mathrm{UK}$, model $\mathrm{DF} \pm 2.5 \mathrm{~mm}$, precisión $\pm 10 \mu \mathrm{m}$ ). Las mediciones se registraron cada $30 \mathrm{~s}$ en un datalogger y un multiplexor (modelo CR1000, Campbell Scientific, Inc., Logan, EE.UU.), los cuales fueron programados para grabar valores medios cada 15 minutos. La máxima contracción diaria del tronco (MCD) se calculó diariamente como la diferencia entre el máximo y mínimo diámetro del tronco (Goldhamer y Fereres, 2001).

La fotosíntesis neta $\left(\mathrm{P}_{\mathrm{n}}\right)$ y la conductancia estomática $\left(\mathrm{g}_{\mathrm{s}}\right)$ se midieron sobre hojas directamente expuestas a la radiación solar en los mismos días que el $\Psi$, usando para ello un sistema portátil de medida de fotosíntesis (LI-6400, Li-Cor, Lincoln, NE, USA).

El diámetro del fruto se midió cada 7-10 días usando un calibre digital (Powerfix model Nr Z22855F, Milomex Ltd, Bedfordshire, UK), y el crecimiento de brotes se obtuvo cada 7-10 días midiendo su longitud.

- $\quad$ El tamaño del fruto se evaluó mediante su producción comercializable o no comercializable, según Regulación Europea (EC) nº 1861/2004.

- $\quad$ El diámetro del tronco se midió anualmente con un pie de rey para estimar el área de la sección transversal del tronco.

- $\quad$ El peso de madera de poda se registró en la poda de invierno en ambos cultivos usando una balanza Scaltec Mod. SSH91, capacidad $150 \mathrm{~kg}$ y precisión $\pm 5 \mathrm{~g}$.

En cosecha, la firmeza, el pH, el contenido en sólidos solubles y la acidez titulable (AT) se evaluaron como índices de calidad del fruto en una muestra representativa de cada tratamiento estudiado (100 frutos por tratamiento). Los análisis de firmeza, $\mathrm{pH}$ y contenido en sólidos solubles se realizaron de acuerdo con Artés et al. (1993), mientras que la acidez titulable se determinó mediante el método de AOAC (1984).

- $\quad$ Los valores de color, en la superficie (color de la piel) y tras pelar (color de pulpa), se midieron con un colorímetro (CR-300, Minolta, Ramsey, NJ). El número de frutos que se usó por tratamiento fue de 200 (50 por parcela experimental) y se realizaron 3 medidas en cada fruto, tanto en la piel como en la pulpa. 
La presente tesis se ha dividido en cuatro capítulos independientes pero relacionados, cuyos objetivos y resumen se exponen a continuación:

\section{Capítulo 5 (REFERENCE VALUES OF MAXIMUM DAILY TRUNK SHRINKAGE FOR IRRIGATION SCHEDULING IN MID-LATE MATURING PEACH TREES) enviado a la revista “Agricultural Water Management”.}

En este capítulo, se obtuvieron medidas de potencial hídrico de tallo $\left(\Psi_{\mathrm{t}}\right)$ y máxima contracción diaria del tronco (MCD) en melocotoneros [Prunus persica (L.) Batsch cv. "Catherine"] durante 3 años consecutivos con el objetivo de obtener ecuaciones de referencia que permitan la programación del riego a partir de medidas automáticas de MCD como herramienta de gestión.

En este ensayo, los árboles se regaron por encima de sus necesidades hídricas (120\% de la $\mathrm{ET}_{\mathrm{c}}$ ) para obtener condiciones no limitantes de agua en el suelo. Se determinaron las relaciones entre la MCD y distintos parámetros climáticos como la $\mathrm{ET}_{0}$, temperatura y déficit de presión de vapor (DPV) (máxima y al mediodía) así como con el $\Psi_{\mathrm{t}}$, un indicador del estado hídrico de la planta ampliamente reconocido.

Nuestros resultados indicaron que los registros de MCD presentaban una mayor correlación con la temperatura, tanto al mediodía como máxima diaria, durante el período de crecimiento del fruto que con las otras variables estudiadas; mientras que en post-cosecha, la mejor relación se obtuvo con el DPV. Las relaciones entre la MCD y $\Psi_{t}$ difirieron interanualmente, aunque se encontraron ecuaciones de referencia similares entre estaciones de crecimiento para los distintos períodos post-cosecha. Sin embargo, estas relaciones variaron mucho con el estado fenológico del árbol y la carga de frutos de cada año. En conclusión, los agricultores deben ser conscientes de estos factores al utilizar valores MCD para obtener ecuaciones de referencia de cara a su utilización en la programación del riego. 
Capítulo 6 (USEFULNESS OF MAXIMUM DAILY TRUNK SHRINKAGE FOR ESTIMATING WATER NEEDS AND REGULATED DEFICIT IRRIGATION SCHEDULING IN PEACH TREES) enviado a la revista "Agricultural Water Management".

A pesar de la facilidad de uso y robustez de las medidas de $\Psi_{\mathrm{t}}$, el inconveniente de tener que desplazarse hasta la finca en numerosas ocasiones es un hecho a tener en cuenta, por ello, en este capítulo, se evaluó la aplicabilidad de la determinación del estado hídrico de la planta mediante sensores de medida de las fluctuaciones del diámetro de los troncos (sensores TDLV), utilizando los valores de la máxima contracción diaria del tronco (MCD) durante 3 años consecutivos en melocotonero [Prunus persica (L.) Batsch cv. "Catherine"].

El objetivo fue, en primer lugar, cuantificar las necesidades hídricas de la planta, a partir de las medidas de $\mathrm{MCD}$, y normalizarlo respecto al control (MCDtrat/MCDControl) como intensidad de señal de la MCD (IS $\mathrm{MCD}_{\mathrm{MC}}$ ), a fin de eliminar el efecto climatológico, manteniéndola en valores cercanos a la unidad durante todo el ciclo de cultivo. Por otro lado, se programó un tratamiento de Riego Deficitario Controlado (RDC) en base a la $\mathrm{IS}_{\mathrm{MCD}}$, manteniendo valores cercanos a 2 durante los períodos no críticos para el desarrollo del cultivo. Así, los tratamientos aplicados fueron:

a) Un control sobrerregado $\left(\mathrm{C}_{\mathrm{ETc}}\right)$ por encima de las necesidades hídricas del cultivo (aproximadamente un $120 \%$ de la $\mathrm{ET}_{\mathrm{c}}$ de media para los 3 años).

b) Un tratamiento de precisión o control ajustado $\left(\mathrm{C}_{\mathrm{MCD}}\right)$ donde se mantuvieron los valores de $\mathrm{IS}_{\mathrm{MCD}}$ en torno a la unidad durante todo el ciclo de cultivo.

c) Un tratamiento RDC donde se mantuvo un valor de IS $_{\mathrm{MCD}}$ en torno a la unidad en todos los estados fenológicos del cultivo excepto en los periodos no críticos (Fase II de crecimiento del fruto y período post-cosecha) donde el valor de la IS $\mathrm{SCD}_{\mathrm{MC}}$ mantuvo en torno a 2 .

La estrategia para alcanzar y mantener estos niveles de estrés fue mediante el protocolo propuesto por Goldhamer y Fereres (2001). 
Los resultados indicaron valores de $\mathrm{MCD}$ e $\mathrm{IS}_{\mathrm{MCD}}$ similares para los tratamientos $\mathrm{C}_{\mathrm{MCD}}$ y $\mathrm{C}_{\mathrm{ETc}}$ durante los 3 años. El tratamiento RDC presentó valores más altos de estos indicadores durante los periodos en los que el déficit hídrico fue impuesto. Los valores de $\mathrm{IS}_{\mathrm{MCD}}$ para el tratamiento $\mathrm{C}_{\mathrm{MCD}}$ presentaron unos coeficientes de variación (CV) bajos, en torno a 0,082; 0,069 y 0,083 para cada uno de los 3 años de experimentación. Por otro lado, en el tratamiento de RDC, dentro de los periodos en los que se aplicó el déficit hídrico, el CV ascendió moderadamente a valores entre 0,119 y 0,324 .

El ahorro hídrico del tratamiento deficitario se situó alrededor del 43-65\% respecto a la $\mathrm{ET}_{\mathrm{c}}$ durante los 3 años de la experiencia y no se observaron diferencias significativas en el crecimiento de los frutos, aunque sí en el crecimiento vegetativo. Los valores del índice de madurez de los frutos se vieron incrementados bajo RDC.

El hecho de que el tratamiento $\mathrm{C}_{\mathrm{MCD}}$ presentara niveles de $\Psi_{\mathrm{t}}$, intercambio gaseoso y MCD similares al control, además de una producción y calidad del fruto sin diferencias significativas, indicó que los melocotoneros bajo este tratamiento de precisión no fueron afectados por el protocolo de riego impuesto, por lo que, en ausencia de drenaje y manteniendo los niveles de IS $_{\text {MCD }}$ cercanos a la unidad se puede considerar como una herramienta plausible para poder estimar los valores de $\mathrm{K}_{\mathrm{c}}$ cuando éstos no se conozcan a nivel local. Por tanto, nuestros resultados confirmaron la viabilidad de programar el riego basándose en medidas de $\mathrm{IS}_{\mathrm{MCD}}$.

Capítulo 7 (USE OF MIDDAY STEM WATER POTENTIAL FOR DEFICIT IRRIGATION SCHEDULING IN PEACH ORCHARDS IN SOUTH-EAST SPAIN) enviado a la revista "Agricultural Water Management".

En este capítulo se procedió a evaluar el efecto del RDC en la respuesta fisiológica y productiva de melocotoneros de media-tardía estación [Prunus persica (L.) Batsch cv. "Catherine"] durante 3 años consecutivos. Para ello, el riego se programó mediante el empleo de valores umbrales establecidos para el potencial hídrico de tallo $\left(\Psi_{t}\right)$ (medidas discontinuas del estado hídrico de la planta), debido a que estudios previos lo sitúan como un indicador robusto, fiable y sensible del estado hídrico de la planta. 
Se establecieron tres tratamientos:

Un control sobrerregado (aproximadamente un $120 \% \mathrm{ET}_{\mathrm{c}}$ de media entre las tres campañas estudiadas), a fin de no tener condiciones limitantes de agua en el suelo, y dos tratamientos deficitarios:

a) RDC I (Déficit moderado) las plantas se regaron a fin de mantener los valores de $\Psi_{\mathrm{t}}$ cercanos a $-1,5 \mathrm{MPa}$ durante la fase II de crecimiento del fruto y en el período postcosecha.

b) RDC II (Déficit severo) las plantas se regaron a fin de mantener los valores de $\Psi_{\mathrm{t}}$ cercanos a $-1,8 \mathrm{MPa}$ durante la fase II de crecimiento del fruto y a $-2,0 \mathrm{MPa}$ en el período postcosecha.

Para mantener estos niveles de déficit hídrico se siguió el protocolo propuesto por Goldhamer y Fereres (2001) para riegos de alta frecuencia. Así, si el valor observado era más alto que el umbral establecido, se reducía el riego un $10 \%$ y, en caso contrario, se aumentaba en el mismo porcentaje.

Nuestros resultados mostraron una reducción en el agua aplicada respecto a la $\mathrm{ET}_{\mathrm{c}}$ de entre 38 y $68 \%$ en los tratamientos deficitarios, lo cual se tradujo en menores valores de contenido volumétrico del agua en el suelo y en valores más negativos de $\Psi_{\mathrm{t}}$ en estos tratamientos, causando también una reducción en los parámetros de intercambio gaseoso $\left(\mathrm{P}_{\mathrm{n}} \mathrm{y} \mathrm{g}_{\mathrm{s}}\right)$. El crecimiento vegetativo y la poda se vieron reducidos en ambos tratamientos de RDC respecto al control, efecto que no se observó en la evolución del tamaño del fruto y la cosecha. Asimismo, se observó una mejora en la calidad de los frutos bajo RDC asociada fundamentalmente a un aumento en el índice de madurez.

Nuestros resultados demostraron que el uso de valores umbrales de $\Psi_{\mathrm{t}}$ para programar el riego es una opción viable para ahorrar agua sin comprometer el rendimiento. La disminución del crecimiento vegetativo observada a lo largo del presente experimento puede ser de interés para los agricultores ya que se podrían ver reducidos los costes de operaciones de poda (menor tiempo de trabajo), así como se obtendría una mejor intercepción de luz en la copa de los árboles. Las características físico-químicas de los frutos indicaron una mejora en la calidad del fruto bajo los 
tratamientos RDC. Sin embargo, estos efectos deben estudiarse a largo plazo para evaluar la sostenibilidad de estas estrategias, así como para adaptar los umbrales $\Psi_{\mathrm{t}}$ a otras condiciones de suelo y clima.

Capítulo 8 (EFFECTS OF REGULATED DEFICIT IRRIGATION ON PHYSIOLOGY, YIELD AND FRUIT QUALITY IN APRICOT TREES) enviado a la revista "Scientia Horticulturae".

En este último capítulo se evaluó el efecto del RDC en albaricoquero [Prunus armeniaca (L.) cv. "Búlida"] durante 3 años consecutivos, calculando los requerimientos hídricos semanalmente según la ecuación de Penman-Monteith (Allen et al., 1998) y un coeficiente local de cultivo $\left(\mathrm{K}_{\mathrm{c}}\right)$, de 0,5 en Febrero; 0,75 en Marzo; 0,8 en Abril; 0,9 en Mayo; 0,6 en Junio y 0,5 desde Julio hasta Noviembre, según indicaron Abrisqueta et al. (2001).

Los tratamientos establecidos fueron dos: Un control regado al $100 \%$ de la $\mathrm{ET}_{\mathrm{c}}$ durante todo el ciclo de cultivo y un tratamiento de riego deficitario controlado (RDC) regado al $100 \%$ de la $\mathrm{ET}_{\mathrm{c}}$ durante los estados fenológicos críticos y cuyo porcentaje de reducción del riego respecto a la $\mathrm{ET}_{\mathrm{c}}$ en los periodos no críticos fue función del estado fenológico de la planta.

Según estudios previos de Torrecillas (2000), estos porcentajes fueron de: a) $40 \%$ de $\mathrm{ET}_{\mathrm{c}}$ desde floración hasta el final de la fase I del crecimiento del fruto; b) $60 \%$ de ET $_{\mathrm{c}}$ durante la fase II de crecimiento del fruto, c) $50 \%$ ET $_{\mathrm{c}}$ durante la post-cosecha tardía I, la cual tiene una extensión de 30 días y comienza 60 días después de la recolección y d) 25\% $\mathrm{ET}_{\mathrm{c}}$ hasta el fin de la defoliación (post-cosecha tardía II).

Los resultados indicaron que el albaricoquero es una especie apropiada para el establecimiento de estrategias de RDC debido a que presenta una clara separación entre el crecimiento vegetativo y reproductivo, así como su capacidad para recuperar la reducción de diámetro de fruto sufrida durante la aplicación del déficit hídrico. El estrés impuesto no redujo significativamente el crecimiento vegetativo ni el área de la sección transversal del tronco en el tratamiento RDC en comparación con el control. En cuanto a la calidad de los frutos, algunas características cualitativas como el índice de madurez 
aumentaron bajo RDC, debido a un incremento en el contenido en sólidos solubles y a la ausencia de efectos sobre la acidez, lo que según estudios previos afecta la percepción del gusto (dulzura y acidez) por el consumidor, influyendo en sus decisiones de compra. Los parámetros de color del fruto también mejoraron en el tratamiento deficitario, siendo mayores los valores de ángulo HUE (matiz), croma (intensidad) y claridad bajo RDC. Estas razones, junto con ahorros en el agua aplicada al tratamiento de RDC de alrededor del $33 \%$ en promedio, demuestran que el RDC es una estrategia de manejo del agua de riego adecuada para las zonas con escasez de recursos hídricos como el sureste español. 


\section{Summary}

Water resources scarcity and low quality, together with the pressure of tourist, industrial and residential developments, makes that agriculture in semiarid regions must deal with water-limiting situations almost permanently. Irrigated agriculture consumes more than $70 \%$ of available water resources causing, in some cases, overexploitation of groundwater resources and the loss of water quality. However, it is difficult to tackle the problem due to several reasons such as:

- $\quad$ The consequences of excessive water use and fertilizers in intensive agriculture are not taken into account at the medium and long terms.

The effective management of water resources is a collective task since its implementation requires the involvement of all the agents, both water resource managers and farmers or end users.

User limited awareness about the dimensions of the problem of water scarcity and its consequences, in some cases.

- $\quad$ Low utilization of tools / techniques to save water in agriculture.

In order to improve this situation, a collective effort between who manages water resources and who uses them is required. The first ones should seek new management policies and participatory governance systems, while the second ones must change their practices to get higher yields and better crop quality with a lower use of water resources.

Farmers, supported by research studies and the introduction of new technologies, can mitigate the consequences of water shortages through several complementary options:

1) Precision irrigation: this strategy seeks to minimize water losses that usually occur during irrigation events. It requires an efficient system and an irrigation scheduling methodology that takes into account water relations in the soil-plantatmosphere system.

2) Deficit irrigation: the fact that crop response to water deficit is variable depending on the plant growth stage allows for reducing water application through 
irrigation during non-critical phenological stages without causing losses in the final yield.

3) Use of unconventional water: municipal reclaimed water represents a considerable volume of total agricultural consumption, thus often occur in nearby agricultural areas. These water resources also contain a high concentration of minerals and organic matter that could partially cover plan nutritional needs.

For these reasons, in recent years the studies that address the implementation of regulated deficit irrigation strategies (RDI) have become very important, due to the water savings they entail, which can even result in qualitative improvements in some aspects of the harvests obtained (Fereres and Soriano, 2007). Based on this, plant based water status measurements have gained interest because they integrate the effect of both the soil water availability and the evaporative demand and, therefore, provide more suitable information for determining the water restrictions effect on plant physiological activity and irrigation scheduling (Jones, 2004). These water status indicators may be distinguished in two groups, if they allow for continuous data collection (maximum daily trunk shrinkage, MDS) or not (stem water potential, $\Psi_{\mathrm{s}}$ ), in order to automate irrigation.

In the world context, peach [Prunus persica (L.) Batsch] is one of the most extended fruit crops, being the second fruit species in production after apple. Its great acceptance by consumers is due to its variety of uses, including fresh consumption. Its main producing country is China, whereas Spain ranks fourth. In the Murcia Region, peach is the first stone fruit in yield and the second one in cultivated surface after almonds. Murcia region, with a cultivated area of 10,713 ha and a production of $171,526 \mathrm{t}$ ranks third in peach production within the country, after Aragon and Catalonia (MAGRAMA, 2015).

In the case of apricot (Prunus armeniaca L.), worldwide production exceeds 2.5 million tons, being Turkey the leading producer, followed by Iran, while Spain occupies the $9^{\text {th }}$ place (FAO, 2013). In Spain, the Murcia region, with approximately $60 \%$ of the national production, ranks first in both production and cultivated area, with a great difference compared to other regions (MAGRAMA, 2015).

For these reasons, the general objectives of this doctoral thesis were to: 
- Obtain reference equations from continuous measurements of trunk diameter fluctuations with plant sensors for irrigation scheduling in adult peach trees [Prunus persica (L.) Batsch cv "Catherine"], by calculating the maximum daily shrinkage (MDS) and relating it to climatic variables and stem water potential.

- Quantify the plant water needs based on the MDS measures as a precision tool for irrigation scheduling maintaining signal intensity values (IS $\mathrm{S}_{\mathrm{MDS}}$, defined as $\mathrm{MDS}_{\text {reference }} / \mathrm{MDS}_{\text {Control }}$ ) close to unity for all the growing season.

- $\quad$ Assess the use of MDS as a plant-based indicator for regulated deficit irrigation (RDI) scheduling, establishing a signal intensity threshold value of 2 during non critical growth periods for this irrigation strategy, and evaluating production and crop quality in adult peach trees [Prunus persica (L.) Batsch cv "Catherine"].

- $\quad$ Assess the use of the midday stem water potential as a plant-based indicator for RDI scheduling, establishing threshold values for this parameter in adult peach trees [Prunus persica $($ L.) Batsch cv "Catherine"].

- $\quad$ Assess the RDI effect in plant water relations, yield and fruit quality in adult apricot trees [Prunus armeniaca (L.) cv. "Búlida"] during three consecutive years.

In order to achieve these objectives, experiments were performed over three consecutive years in a commercial orchard located in Mula (Murcia) $\left(37^{\circ} 55^{\prime} \mathrm{N}, 1^{\circ} 25^{\prime} \mathrm{W}\right.$, $360 \mathrm{~m}$ above sea level). The climate is typically Mediterranean, with mild winters, hot dry summers and low rainfall amounts (between 188 and $304 \mathrm{~mm}$ per year over the three years of study). Two plots were selected within the same orchard. One of 0.5 ha of peach [Prunus persica (L.) Batsch] cv. "Catherine" (mid-late season), grafted on a hybrid pattern peach $\mathrm{x}$ almond GF-677 rootstock, 9 years old at the beginning of the experience (2008), spacings of $4 \mathrm{~m} \mathrm{x} 6 \mathrm{~m}$. The soil at the site is calcareous, stony, with a sandy-loam texture, low organic matter content and its available water capacity is 0.25 $\mathrm{m}^{3} \mathrm{~m}^{-3}$, approximately.

On the other hand, a 1-ha plot of apricot trees [Prunus armeniaca L.] cv. "Búlida", grafted on Real fino apricot rootstock, and 9 years old at the beginning of the experience, spacings of $8 \mathrm{~m} \times 6 \mathrm{~m}$. The orchard soil is highly calcareous with a clay- 
loam texture, low organic matter content and available water capacity is about $0.31 \mathrm{~m}^{3}$ $\mathrm{m}^{-3}$ aproximately.

In both cases, irrigation water came from a nearby water well and had electrical conductivity values around $0.6 \mathrm{dS} \mathrm{m}^{-1}$.

The measurements carried out over the experimental period were:

- $\quad$ Climate data were recorded at an automatic weather station placed within the experimental orchard.

- The volumetric soil water content $\left(\theta_{\mathrm{v}}, \mathrm{m}^{3} \mathrm{~m}^{-3}\right)$ was measured by time domain reflectometry (TDR) (Tektronix device (Model 1502C, Tektronix Inc., OR), as described by Moreno et al. (1996), and a neutron probe (Model 4300, Troxler Electronic Laboratories Inc., NC), measurements were taken in the morning, every 7 to 15 days, during the experimental period.

- $\quad$ Midday stem water potential $\left(\Psi_{\mathrm{s}}\right)$ and leaf water potential $\left(\Psi_{1}\right)$ were measured using a pressure chamber (Soil Moisture Equip. Corp, model 3000, Santa Barbara, CA, USA) following the procedures described by Turner (1981).

- The micrometric trunk diameter fluctuations (TDF) were measured throughout the experimental period using a set of linear variable displacement transducers (LVDT) (Solartron Metrology, Bognor Regis, UK, model DF $\pm 2.5 \mathrm{~mm}$, accuracy $\pm 10 \mu \mathrm{m}$,) Measurements were taken every $30 \mathrm{~s}$ with a datalogger and a multiplexor (model CR1000, Campbell Scientific, Inc., Logan, USA), which were programmed to report 15 min means. Maximum daily trunk shrinkage (MDS) was calculated daily as the difference between the maximum and minimum daily trunk diameter (Goldhamer and Fereres, 2001).

- $\quad$ Net photosynthesis $\left(\mathrm{P}_{\mathrm{n}}\right)$ and stomatal conductance $\left(\mathrm{g}_{\mathrm{s}}\right)$ were measured in sun-facing leaf in the same days that stem water potential was recorded, using a fieldportable photosynthesis system (LI-6400, Li-Cor, Lincoln, NE, USA).

Fruit diameter was measured every 7-10 days using a digital caliper (Powerfix model Nr Z22855F, Milomex Ltd, Bedfordshire, UK) and shoot length values were collected every 7-10 days by measuring their lengths. 
- $\quad$ Fruit size was studied by evaluating marketable and non marketable production according to Commission Regulation (EC) n 1861/2004

Trunk diameter was measured annually with a sliding caliper and used to estimate trunk cross-sectional area.

- $\quad$ Pruning weight was recorded at winter pruning in both crops using a scale (Scaltec Mod. SSH91, capacity $150 \mathrm{~kg}$ and accuracy $\pm 5 \mathrm{~g}$ ).

- $\quad$ At harvest, firmness, $\mathrm{pH}$, soluble solids content (SSC) and titratable acidity (TA) were evaluated as fruit quality indices in a representative sample of each treatment studied (100 fruits per treatment). Firmness, pH and SSC determinations were conducted according to Artés et al. (1993), while titratable acidity (TA) was determined by AOAC (1984).

Colour values, on the surface (ground skin colour) and after peeling in the flesh, were measured with a Minolta chromameter (CR-300, Minolta, Ramsey, NJ). The number of fruits used per treatment was 200 (50 per experimental plot) and 3 measurements were taken per fruit, both in skin and pulp.

This thesis has been divided into four independent but related chapters, their objectives and summaries are presented below:

\section{Chapter 5 (REFERENCE VALUES OF MAXIMUM DAILY TRUNK SHRINKAGE FOR IRRIGATION SCHEDULING IN MID-LATE MATURING PEACH TREES) submitted to "Agricultural Water Management".}

In this chapter, measures of stem water potential $\left(\Psi_{\mathrm{s}}\right)$ and maximum daily trunk shrinkage (MDS) were obtained for peach [Prunus persica (L.) Batsch cv. "Catherine"] over 3 consecutive years aiming to obtain reference equations that allow for irrigation scheduling based on automatic measurements of MDS as a management tool. In this experiment, the trees were irrigated over their water needs $\left(120 \% \mathrm{ET}_{\mathrm{c}}\right)$ for obtaining non-limiting water conditions on the soil. Relations between the MDS and several climatic parameters such as $\mathrm{ET}_{0}$, temperature and vapor pressure deficit (VPD) (maximum and at midday) were obtained, as well as with the $\Psi_{\mathrm{s}}$, an indicator of plant 
water status widely recognized. Our results indicated that, during the fruit growth period, MDS records showed a higher correlation with temperature, either at midday or the daily maximum, than with the other variables studied; while at postharvest, the best correlation value was obtained with VPD. Relations between the MDS and $\Psi$ s differed between years, although similar reference equations between growing seasons were found for the different postharvest periods. However, these relationships varied considerably with the growth stage of the tree and fruit load each year. In conclusion, farmers must be aware of these factors when using MDS values for getting reference equations in order to use them in irrigation scheduling.

Chapter 6 (USEFULNESS OF MAXIMUM DAILY TRUNK SHRINKAGE FOR ESTIMATING WATER NEEDS AND REGULATED DEFICIT IRRIGATION SCHEDULING IN PEACH TREES) submitted to "Agricultural Water Management".

Despite the ease of use and robustness of $\Psi_{\mathrm{s}}$ measures, the inconvenience of having to go to the farm on a great number of occasions is a fact to consider. Therefore, in this chapter, the applicability of plant water status determination by means of trunk diameter fluctuations sensors (LVDT sensors) was evaluated over three years in peach [Prunus persica (L.) Batsch cv. "Catherine"] using maximum daily trunk shrinkage (MDS) records.

The aim of this study was, first, to quantify the plant water needs from MDS measures and standardize them respect to control (MDStreat / MDSControl) as MDS signal intensity (MDS SI), in order to eliminate the climatic effect, keeping its values close to unity throughout the growing season. Furthermore, regulated deficit irrigation treatment (RDI) was scheduled based on MDS SI, keeping its values close to 2 during non critical periods for crop development. Thus, the treatments were:

a) A Control $\left(\mathrm{C}_{\mathrm{ETc}}\right)$ irrigated over the crop water needs $\left(120 \%\right.$ of $\mathrm{ET}_{\mathrm{c}}$ averaged for the three years, aproximately).

b) A precision treatment or adjusted control ( $\left.\mathrm{C}_{\mathrm{MDS}}\right)$ where MDS SI values around unity were maintained throughout the growing season. 
c) A RDI treatment where MDS SI values were maintained around unity in all developmental stages of crop except for noncritical periods (stage II of fruit growth and postharvest period) where the MDS SI values were kept around 2.

The strategy to achieve and maintain these stress levels was based on that proposed by Goldhamer and Fereres (2001).

The results indicated similar MDS and MDS SI values for $\mathrm{C}_{\mathrm{ETc}} \mathrm{C}_{\mathrm{MDS}}$ treatments during the 3 years. The RDI treatment showed higher values of these indicators during the periods when the water deficit was imposed. MDS SI values for the $\mathrm{C}_{\text {MDS }}$ treatment showed low coefficients of variation (CV), around $0.082 ; 0.069$ and 0.083 for each of the three years of experimentation, respectively. On the other hand, in the RDI treatment, the CV moderately increased between 0.119 and 0.324 within the periods in which the water deficit was applied.

The water savings in the RDI treatment were around $43-65 \%$ respect to $\mathrm{ET}_{\mathrm{c}}$ during the 3 years of experience and no significant differences were observed in fruit growth, although they were observed in vegetative growth. Fruit maturity index values increased under RDI. The fact that the $\mathrm{C}_{\mathrm{MDS}}$ treatment present $\Psi_{\mathrm{s}}$, gas exchange and MDS levels similar to those of the control treatment, as well as yield and fruit quality without significant differences, indicated that the peaches under this precision treatment were not affected by the irrigation protocol imposed, so that maintaining levels of MDS SI close to unity can be considered as a plausible tool, in the absence of drainage, to estimate the $K_{c}$ values when they are not locally available. Therefore, our results confirmed the viability of irrigation scheduling based on MDS SI measurements.

\section{Chapter 7 (USE OF MIDDAY STEM WATER POTENTIAL FOR DEFICIT} IRRIGATION SCHEDULING IN PEACH ORCHARDS IN SOUTH-EAST SPAIN) submitted to "Agricultural Water Management".

In this chapter, we assessed the effect of the RDI in the physiological and yield response of mid-late season peach [Prunus persica (L.) Batsch cv. "Catherine"] during three consecutive years. For this, irrigation was programmed through stem water potential $\left(\Psi_{\mathrm{s}}\right)$ (discontinuous measurement of plant water status) threshold values, since 
previous studies consider it a robust, reliable and sensitive indicator of plant water status.

Three treatments were established:

An over irrigated control (120\% $\mathrm{ET}_{\mathrm{c}}$ averaged among the three seasons studied), in order to obtain non-water-limiting conditions on soil, and two deficitary treatments:

a) RDI I (moderate deficit) plants were irrigated to keep $\Psi_{\mathrm{s}}$ values close to 1.5 MPa during stage II of fruit growth and postharvest period.

b) RDI II (severe deficit) plants were irrigated to keep $\Psi_{\mathrm{s}}$ values close to 1.8 $\mathrm{MPa}$ during stage II of fruit growth and -2.0 $\mathrm{MPa}$ at postharvest period.

To maintain these levels of water deficit the protocol proposed by Goldhamer and Fereres (2001) for high frequency irrigation was followed. Thus, if the value observed was higher than the threshold, irrigation was reduced by $10 \%$, otherwise it was increased in the same percentage.

Our results showed a reduction in water applied between 38 and $68 \%$ in the RDI treatments as compared to the $\mathrm{ET}_{\mathrm{c}}$, which resulted in lower values of volumetric soil water content and $\Psi_{\mathrm{s}}$ more negative in these treatments, also causing a reduction in gas exchange parameters $\left(\mathrm{P}_{\mathrm{n}}\right.$ and $\left.\mathrm{g}_{\mathrm{s}}\right)$. Vegetative growth and pruning were reduced in both RDI treatments compared to the control, whereas no effects were observed in the fruit growth evolution and harvest. Moreover, an improvement was observed in fruit quality under RDI, fundamentally associated with a maturity index increase. Our results showed that the use of $\Psi_{\mathrm{s}}$ threshold values to schedule irrigation is a viable option for saving water without compromising crop yield. The decrease of vegetative growth observed throughout this experiment may be of interest to farmers since it could reduce costs of pruning (less working time), as well as allow for a better light interception in trees canopy. The physicochemical characteristics of the fruits indicated improvements in fruit quality under the RDI treatments. However, these effects should be studied in the long-term to assess the sustainability of these strategies, and to adapt $\Psi_{\mathrm{s}}$ thresholds to other soil and climate conditions. 


\section{Chapter 8 (EFFECTS OF REGULATED DEFICIT IRRIGATION ON} PHYSIOLOGY, YIELD AND FRUIT QUALITY IN APRICOT TREES) submitted to "Scientia Horticulturae".

In this final chapter, the RDI effects on apricot [Prunus armeniaca (L.) cv. "Búlida"] were evaluated. Irrigation requirements were calculated weekly according to the Penman-Monteith equation (Allen et al., 1998), and a local crop coefficient $\left(\mathrm{K}_{\mathrm{c}}\right), 0.5$ February, 0.75 March, 0.8 April, 0.9 May, 0.6 June, 0.5 July-November as suggested by Abrisqueta et al. (2001).

Two irrigation treatments were applied: A Control irrigated to $100 \% \mathrm{ET}_{\mathrm{c}}$ during all crop growth stages and a regulated deficit irrigation treatment (RDI) irrigated at $100 \% \mathrm{ET}_{\mathrm{c}}$ during the critical phenological periods and with water restrictions respect to $\mathrm{ET}_{\mathrm{c}}$ during the non-critical periods depending on plant growth stage. According to Torrecillas (2000) the percentages of applied water were: a) $40 \%$ of $\mathrm{ET}_{\mathrm{c}}$ from flowering until the end of the first stage of fruit growth; b) $60 \%$ of $\mathrm{ET}_{\mathrm{c}}$ during the second stage of fruit growth, c) $50 \% \mathrm{ET}_{\mathrm{c}}$ during the late postharvest $\mathrm{I}$, with a duration of 30 days and that starts 60 days after harvesting and d) $25 \%$ of $\mathrm{ET}_{\mathrm{c}}$ until the end of tree defoliation (late postharvest II).

The results indicated that the apricot is an appropriate species to establish RDI strategies because it presents a clear separation between vegetative and reproductive growth and its ability to recover from the suffered fruit diameter reduction during water shortage implementation. Our results showed that the stress imposed did not significantly reduce vegetative growth or trunk cross-sectional area when compared to control. Regarding fruit quality, some qualitative characteristics, such as the maturity index, which according to previous studies affects the taste perception (sweetness and acidity) by the consumer, influencing their buying decisions, improved due to an increase in soluble solids content and the absence of effects on acidity. The fruit colour parameters also improved under the deficit treatment, being the hue angle, chroma (intensity) and lightness values higher under RDI. These reasons, as well as average applied water savings about $33 \%$ show that RDI strategies are an adequate irrigation management tool in areas with water shortages, as the southeast of Spain. 


\section{References / Bibliografía}

Abrisqueta, J.M., Ruiz A., Franco J.A., 2001. Water balance of apricot trees (Prunus armeniaca L. cv. 'Búlida') under drip irrigation. Agricultural Water Management 50, 211227.

Allen, R.G., Pereira, L.S., Raes, D., Smith, M., 1998. Crop evapotranspiration. Guidelines for computing crop water requirements. FAO Irrigation and Drainage paper No 56, Rome, Italy. 300 pp.

AOAC, 1984. Association of Official Analytical Chemists. Official methods of analysis, 15th edn. AOAC, Washington DC.

Artés, F., Escriche, A.J., Martínez, J.A., Marín, J.G., 1993. Quality factors in four varieties of melon (Cucumis melo L.). J. Food Qual. 16, 91-100.

CARM, 2009. Estadística agraria de Murcia. Consejería de Agricultura y Agua de la Región de Murcia. www.carm.es

FAO, 2013. faostat.fao.org

Fereres, E., Soriano, M.A., 2007. Déficit irrigation for reducing agricultural water use. Journal of Experimental Botany. 58, 147-159.

Goldhamer, D.A., Fereres, E., 2001. Irrigation scheduling protocols using continously recorded trunk diameter measurements. Irrigation Science. 20, 115-125.

Jones, H., 2004. Irrigation scheduling: advantages and pitfalls of plant-based methods. Journal of Experimental Botany 55, 2427-2436.

MAGRAMA, 2015. Anuario de estadística Avance 2014. Ministerio de Agricultura, Alimentación y Medio Ambiente.

Moreno, F., Fernández, J.E., Clothier, B.E., Green, S.R., 1996. Transpiration and root water uptake by olive trees. Plant Soil 184, 85-96.

Roussos P.A, Sefferou V, Denaxa N.K, Tsantili E, Stathis V., 2011. Apricot (Prunus armeniaca L.) fruit quality attributes and phytochemicals under different crop load. Scientia Horticulturae 129: 472-478.

Torrecillas A., Domingo R., Galego R., Ruiz-Sánchez M.C., 2000. Apricot tree response to irrigation with holding at different phenological periods. Scientia Horticulturae 85, 201-215.

Turner, N.C., 1981. Techniques and experimental approaches for the measurement of plant water status. Plant Soil 58, 339-366. 
OBJETIVOS/OBJECTIVES 



\section{Objetivos}

Los objetivos generales de esta tesis fueron los siguientes (entre paréntesis se indica el capítulo en el que se abordan):

1. Obtener ecuaciones de referencia para su uso en la programación de riego en melocotoneros adultos [Prunus persica (L.) Batsch cv "Catherine"] a partir de sensores en planta que miden las variaciones continuas de las fluctuaciones del diámetro del tronco, calculando para ello la máxima contracción diaria del tronco (MCD), y relacionándola con las variables climáticas y también con el potencial hídrico de tallo (Capítulo 5).

2. Cuantificar las necesidades hídricas de la planta en base a medidas de la MCD, como herramienta de precisión en la programación de riego, manteniendo los valores de intensidad de señal ( $\mathrm{S}_{\mathrm{MCD}}$, definido como MCD referencia/MCD Control) cercanos a la unidad durante toda la estación de crecimiento (Capítulo 6).

3. Evaluar el uso de la MCD como indicador en planta para la programación del RDC, estableciendo para ello un umbral de la IS de 2 durante los períodos de crecimiento no críticos para el desarrollo del cultivo, evaluando para ello la producción y calidad de cosecha en melocotoneros adultos [Prunus persica (L.) Batsch cv "Catherine”] (Capítulo 6).

4. Utilización del potencial hídrico de tallo a mediodía como indicador en planta para la programación del riego deficitario controlado (RDC), estableciendo para ello diferentes umbrales del mismo en los períodos fenológicos no críticos para el desarrollo de melocotoneros adultos [Prunus persica (L.) Batsch cv "Catherine"], evaluando su respuesta productiva y calidad de cosecha (Capítulo 7).

5. Evaluar la aplicación de un protocolo de RDC en las relaciones hídricas de la planta, producción y calidad del fruto en albaricoqueros adultos [Prunus armeniaca (L.) cv. "Búlida"] durante tres años consecutivos, cuantificando los ahorros hídricos alcanzables (Capítulo 8). 


\section{Objectives}

The main objectives of this doctoral thesis were the following (the chapter where they are addressed is indicated between parentheses):

1. To obtain reference equations from continuous measurements of trunk diameter fluctuations using plant sensors for irrigation scheduling in adult peach trees [Prunus persica (L.) Batsch cv "Catherine"], calculating the maximum daily shrinkage (MDS) and relating it to climatic variables and also with the stem water potential (Chapter 5).

2. To quantify the plant water needs based on the MDS measurements as a precision tool for irrigation scheduling, by maintaining signal intensity values (IS $\mathrm{SDS}_{\text {, }}$ defined as $\mathrm{MDS}_{\text {reference }} / \mathrm{MDS}_{\text {Control }}$ ) close to unity for the whole growing season (Chapter 6).

3. To assess the use of MDS as a plant-based indicator for regulated deficit irrigation (RDI) scheduling, establishing a signal intensity threshold value of 2 for this irrigation strategy during non critical periods of crop development, and evaluating production and fruit quality in adult peach trees [Prunus persica (L.) Batsch cv "Catherine"] (Chapter 6).

4. To use the midday stem water potential as a plant-based indicator for RDI scheduling, by establishing different threshold values for this parameter in adult peach trees [Prunus persica (L.) Batsch cv "Catherine"] and evaluating production and fruit quality (Chapter 7).

5. To assess the RDI effects on plant water relations, yield and fruit quality in adult apricot trees [Prunus armeniaca (L.) cv. "Búlida"] during three consecutive years, also quantifying the water savings obtained by the use of this RDI strategy (Chapter 8). 
ANTECEDENTES 



\section{Antecedentes}

\subsection{Situación actual de los frutales en el mundo, España y la Región de Murcia}

A nivel mundial, en el año 2012, existían más de 324 millones de hectáreas equipadas para el riego, de las cuales, aproximadamente 275 millones (85\%), se encuentran regadas activamente. Esto supone un $20 \%$ de la superficie total cultivada, constituyendo aproximadamente un $40 \%$ de la producción agrícola total, lo que da fe de su gran importancia (FAO, 2015). En nuestro país las diferencias son aún mayores, contando el regadío poco más del $21 \%$ de la superficie total cultivada (aproximadamente 3,6 millones de hectáreas), y aportando más del 55\% de la producción agraria total. En la Región de Murcia la importancia del sector agrícola está basada casi en su totalidad en el regadío, ya que con algo menos del $39 \%$ de la superficie cultivada, genera un 94\% de la producción final (MAGRAMA, 2015a).

En nuestro país, la superficie total de frutales no cítricos (frutales de pepita y hueso) en regadío representa aproximadamente 273.000 ha (32\% de la superficie total cultivada) de las que, en términos de producción, representa más del 75\% de la fruta producida. (MAGRAMA, 2015a). El melocotonero y el nectarino en nuestro país ocupan una superficie de 84.378 ha, prácticamente en su totalidad bajo regadío (95\%), y producen algo más de 1,3 millones de toneladas anuales, aproximadamente el 34\% de la producción total de frutales (MAGRAMA, 2015b). El segundo frutal en superficie de cultivo es el manzano (30.794 ha) seguido por el peral (24.243 ha) con una producción de 545.992 y 425.560 toneladas respectivamente, (14\% y $11 \%$ respecto del total producido). Por último, el albaricoquero y el ciruelo, con una superficie de plantación de 20.334 y 16.614 ha respectivamente, alcanzan un porcentaje de plantación en torno al 3,5 y $4,5 \%$ respectivamente (131.776 y 172.352 toneladas cada uno).

En el contexto mundial, el melocotonero es uno de los cultivos más extendidos, siendo la segunda especie frutal en importancia según su producción después del manzano. Su gran aceptación por el consumidor es debida a su diversidad de usos y a su consumo en fresco.

El melocotonero se encuadra botánicamente dentro de la Familia Rosaceae, género Prunus y especie Prunus persica (L.) Batsch. Es originario de China, donde las referencias de su cultivo se remontan a 3.000 años a.C., extendiéndose probablemente a 
Persia a través de las rutas comerciales por las montañas, llegando a ser conocidos allí como fruta pérsica, de ahí su nombre científico. Hacia el año 330 a.C., su cultivo llegó a Grecia, y durante la Edad Media se extendió por toda Europa. Fue introducido en España por los romanos, siendo al principio un cultivo marginal, pero en el siglo XIX comienza su expansión.

El principal país productor es China, seguido de lejos por Italia, Egipto, España, EE.UU y Turquía. En la Región de Murcia el melocotonero es el primer frutal de hueso en producción y el segundo en superficie de cultivo tras el almendro.

La oferta de melocotón fresco que presenta nuestro país supera los 6 meses de duración, desde finales de abril hasta octubre o primeros de noviembre. Con una superficie cultivada de 10.713 ha y una producción de 171.526 t, la Región de Murcia ocupa el tercer lugar en producción de melocotón dentro del territorio nacional, tras Aragón y Cataluña (Figura 4.1, MAGRAMA, 2015b).

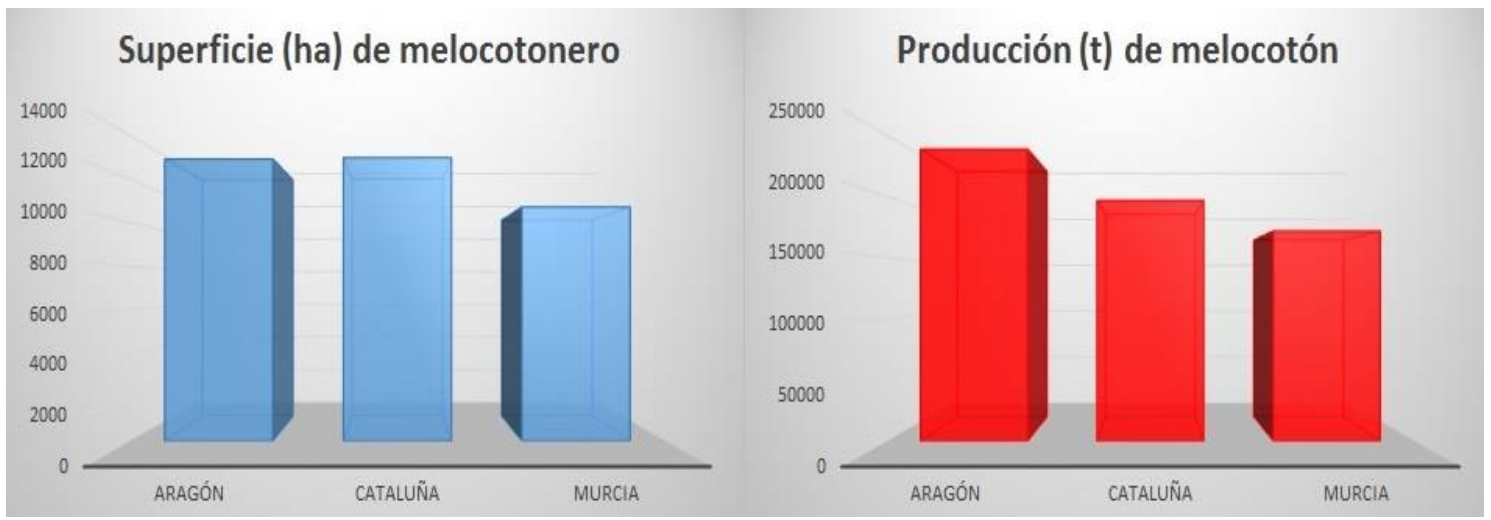

Figura 4.1. Principales Comunidades Autónomas españolas en superficie y producción de melocotones (Datos extraidos de MAGRAMA 2015b).

Las distintas variedades de melocotonero van desde extratempranas, como "Flordastar", árbol de porte semi-erecto, vigoroso con una elevada intensidad de floración cuya recolección tiene lugar la segunda semana de mayo; hasta variedades tardías que se recolectan a partir del 10 de septiembre llegando, incluso, a finales de Noviembre y entre las que destaca la variedad "Melocotón de Calanda".

Una variedad de media estación es "Catherine" que presenta una doble aptitud, siendo su destino principal el mercado en fresco, pero en años de desequilibrio entre la oferta y la demanda, normalmente coincidente con años de fuerte producción, es aceptada por la industria para su procesado. Ha tenido un gran prestigio en el pasado porque está bien adaptada a las condiciones climáticas de la Región de Murcia. Se trata 
de un árbol muy vigoroso y productivo, de fruto medio y redondeado, color amarilloanaranjado, textura bastante firme, buena calidad gustativa, cuya recolección se realiza en la primera quincena de julio (Fotografía 4.1).

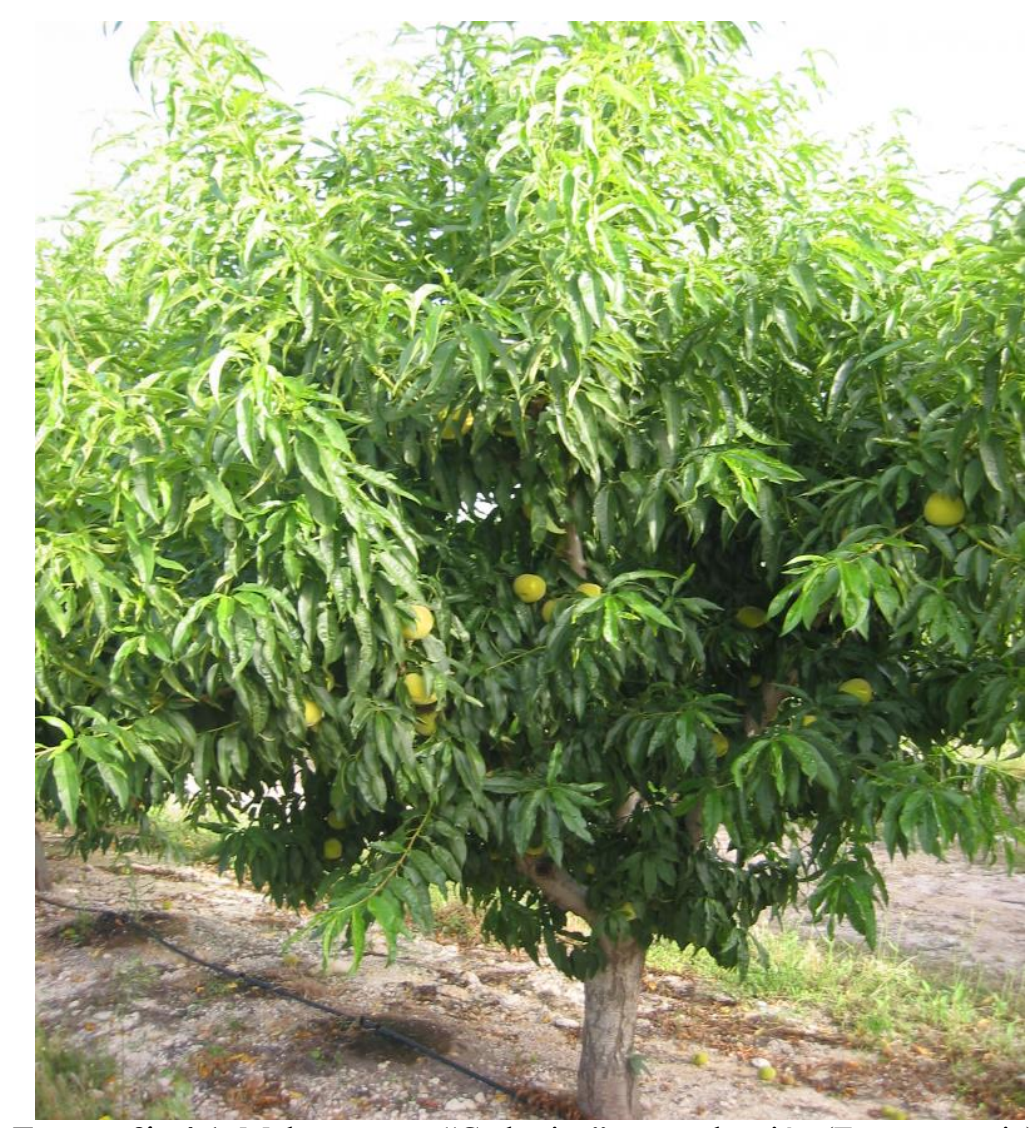

Fotografía 4.1. Melocotonero "Catherine” en producción (Fuente propia).

En el caso del albaricoquero su producción mundial supera las 2,500.000 t, siendo Turquía el principal país productor seguido de Irán. En España, la Región de Murcia, con aproximadamente un $60 \%$ de la producción nacional, ocupa el primer lugar, tanto en producción como en superficie cultivada, con una gran diferencia respecto al resto de Comunidades Autónomas (Figura 4.2, MAGRAMA, 2015b).

El albaricoque es originario del noreste de China hacia 3000 años a.C, cerca de la frontera con Rusia, y no de Armenia como indica su nombre científico, aunque Armenia tiene una gran tradición en su cultivo. Los romanos lo introdujeron en Europa por Anatolia alrededor del año 70 a. C. llamándole Praecox pues florecía temprano, en la primavera. Este cultivo se encuadra botánicamente dentro de la Familia Rosaceae, subfamilia Prunoideae, genero Prunus y especie Prunus armeniaca L. 


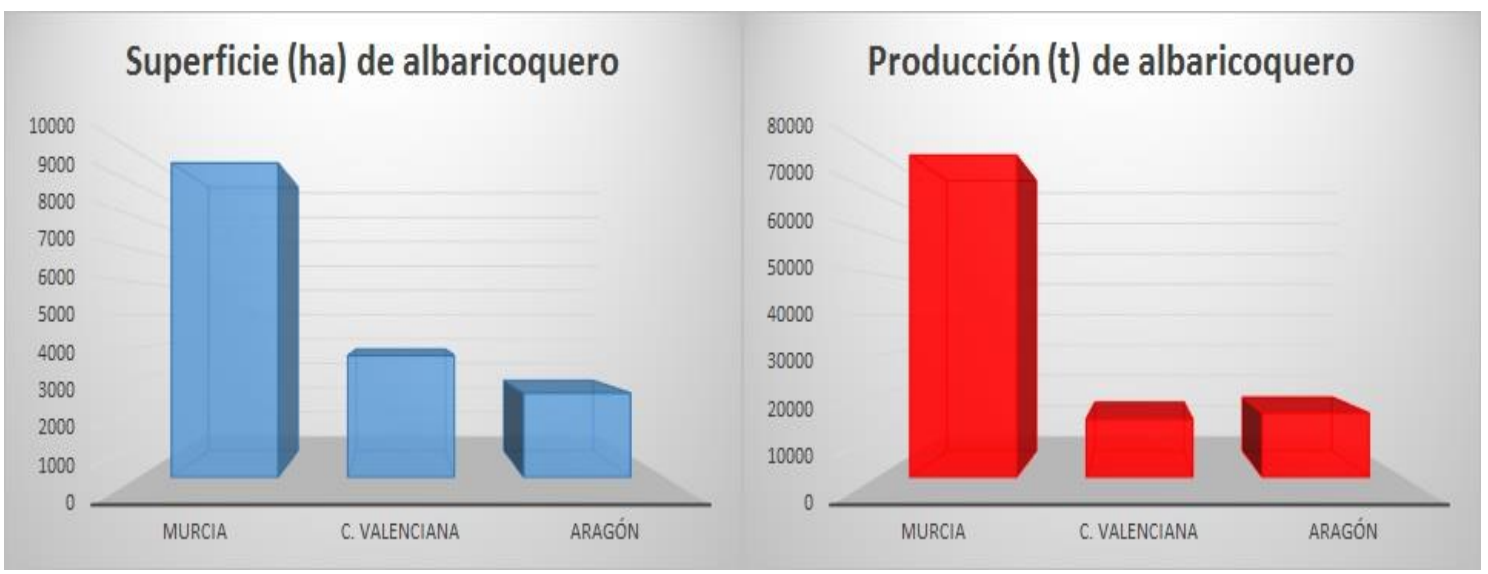

Figura 4.2. Principales Comunidades Autónomas españolas en superficie y producción de albaricoque (Datos extraidos de MAGRAMA 2015b).

El 66\% de la superficie de albaricoquero en la Región de Murcia es de la variedad "Búlida" (CARM, 2009). Se trata de una variedad rústica española que se adapta a todo tipo de suelos. Los árboles son muy vigorosos, de producción abundante y regular, sensibles a oidio y monilia, resistentes al viento y a la caída del fruto (Fotografía 4.2.). Los frutos son grandes y poseen un surco poco profundo, siendo su piel amarilla y de pulpa dulce, bastante jugosa y perfumada. El hueso es grande y tiene quilla. La recolección se lleva a cabo a primeros de junio y presenta una buena conservación.

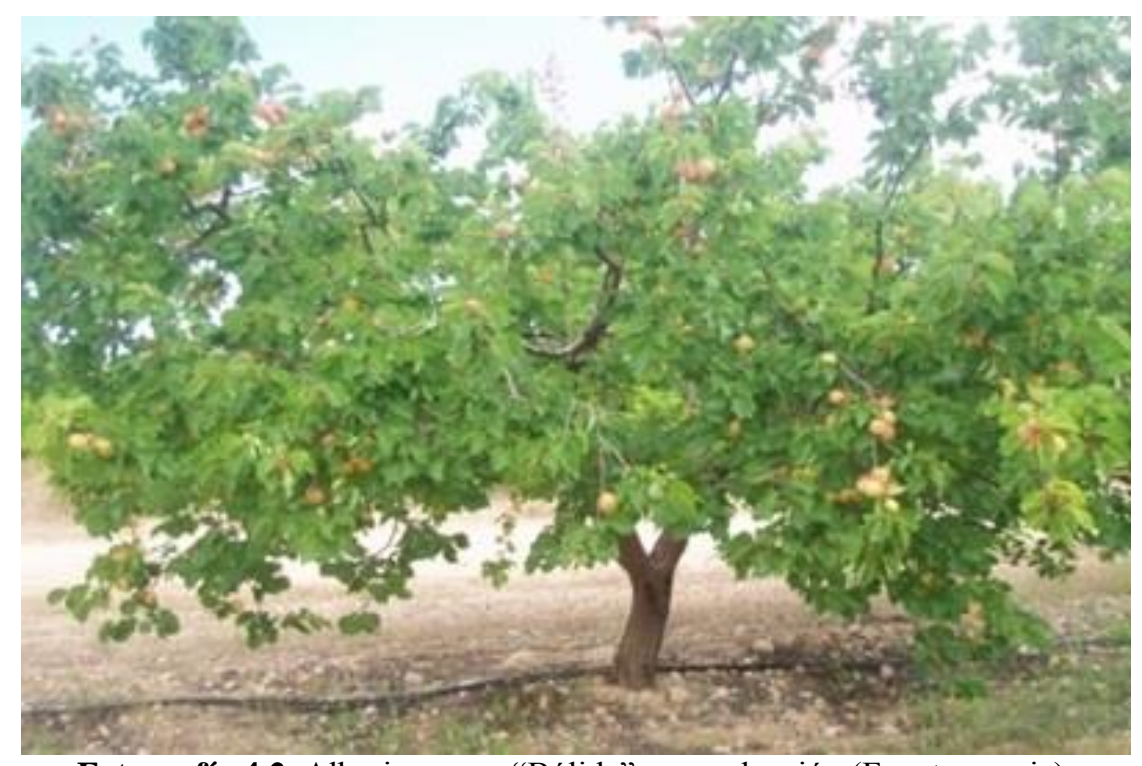

Fotografía 4.2. Albaricoquero "Búlida" en producción (Fuente propia).

Dada la actual expansión en la Región de Murcia de la variedad de melocotón "Catherine" y la variedad "Búlida" en albaricoque, se ha considerado interesante dedicar la presente tesis doctoral a estas dos variedades de frutales de hueso. 


\subsection{Riego de frutales: metodologías}

En el área mediterránea el agua es, sin lugar a dudas, el factor más limitante de la producción de frutas y hortalizas. Esta escasez de recursos hídricos impulsa la necesidad de adoptar estrategias de riego capaces de reducir los aportes de agua aumentando la eficiencia de su uso. Este hecho es de gran importancia en el sector agrario ya que éste utiliza el 75\% de estos recursos hídricos (Libro Blanco del Agua en España, 2008). Aunque en la última década la sociedad se ha concienciado de la necesidad de mejorar la gestión y la protección del agua, los criterios económicos y los factores políticos todavía tienden a dirigir todos los ámbitos de la política del agua, no recibiendo la ciencia y las buenas prácticas la atención adecuada.

El término programación del riego describe el procedimiento mediante el cual se determinan la frecuencia y dosis de agua a aplicar en cada riego (Hillel, 1990). Con el fin de poder planificar esta programación, es necesario conocer las necesidades hídricas de los cultivos, es decir, la cantidad de agua que requieren para un desarrollo productivo máximo. Por tanto, es necesario introducir el término de evapotranspiración (ET).

La ET engloba dos procesos que ocurren simultáneamente:

La evaporación es la cantidad de agua que, por un proceso de vaporización, pasa del suelo a la atmósfera. Al inicio de la estación del crecimiento, el valor de la evaporación es significativamente más alto que en etapas subsiguientes. La transpiración es la cantidad de agua que pasa de la planta a la atmósfera por un proceso de evaporación. A efectos prácticos estos dos componentes se determinan conjuntamente. El conocimiento de la evapotranspiración de los cultivos es esencial para un uso eficiente del agua de riego. Su determinación en plantaciones de frutales es complicada debido a las interacciones existentes entre los diferentes procesos fisiológicos y a la influencia de un gran número de parámetros como las condiciones del medio, técnicas culturales, edad de la plantación, sistema de riego e incluso combinación variedad/patrón (Cohen, 1994).

\section{Evapotranspiración del cultivo de referencia $\left(\mathbf{E T}_{\mathbf{0}}\right)$}

La FAO (Food and Agriculture Organization) definió la evapotranspiración del cultivo de referencia $\left(\mathrm{ET}_{0}\right)$ como el consumo de agua de una superficie extensa de gramíneas, uniforme, de 8 a $15 \mathrm{~cm}$ de altura, en crecimiento activo, que sombrea la 
totalidad del suelo y bien provista de agua (Doorenbos y Pruitt, 1977). Posteriormente y debido a la gran variedad de gramíneas y prácticas de cultivo, este organismo propuso cambiar el concepto de cultivo de referencia y revisar los métodos de cálculo. De este modo, la $\mathrm{ET}_{0}$ en casos en que la vegetación no cubra totalmente el suelo, el factor de resistencia debe incluir los efectos de la evaporación de la superficie del suelo. Si el cultivo no está transpirando de manera potencial, la resistencia depende también del estado del agua dentro de la planta. Para estos casos $\mathrm{ET}_{0}$ se define como la tasa de evapotranspiración de un cultivo hipotético de altura fija de $12 \mathrm{~cm}$, y una resistencia aerodinámica de $208 / \mathrm{U}_{2}\left(\mathrm{~s} \mathrm{~m}^{-1}\right)$, donde $\mathrm{U}_{2}$ es la media de la velocidad del viento medida a $2 \mathrm{~m}$ de altura sobre el dosel vegetal.

Dicha estima puede realizarse bajo condiciones estándar o no estándar. El primer caso hace referencia a la lámina de agua necesaria para cubrir las pérdidas por evapotranspiración en un cultivo sano bajo condiciones óptimas de nutrición y creciendo en lugar amplio. Así, la evapotranspiración del cultivo se estimaría mediante el uso de un coeficiente específico $\left(\mathrm{K}_{\mathrm{c}}\right)$, que depende fundamentalmente del tipo de cultivo, edad del mismo, humedad de la superficie del suelo y fracción de cubierta vegetal (Dorenbos y Pruitt, 1977).

\section{$\operatorname{ET}_{c}\left(\mathbf{m m ~ d}^{-1}\right)=\operatorname{ET}_{0}\left(\mathbf{m m ~ d}^{-1}\right) \cdot K_{c}$}

Bajo condiciones no estándar, la $\mathrm{ET}_{\mathrm{c}}$ se calcularía usando un coeficiente de estrés hídrico $\left(\mathrm{K}_{\mathrm{s}}\right)$ o ajustando el $\mathrm{K}_{\mathrm{c}}$ a las condiciones de estrés y limitaciones ambientales en la evapotranspiración del cultivo.

A continuación se describen brevemente las principales metodologías que se emplean para calcular las necesidades hídricas de los cultivos:

\subsubsection{Métodos climatológicos}

A pesar de la existencia de varias ecuaciones empíricas o semiempíricas para determinar la $\mathrm{ET}_{0}$ usando datos meteorológicos, algunas son sólo válidas para determinadas condiciones climáticas y agronómicas, no extrapolables a condiciones diferentes de las que fueron obtenidas. Se pueden diferenciar los métodos basados en la temperatura del aire, radiación solar o los combinados, que son los más empleados a nivel mundial. 


\section{Métodos basados en la temperatura del aire}

En muchas regiones del planeta, los datos meteorológicos y climatológicos son muy escasos o limitados. En estos casos los modelos de cálculo de la ET basados simplemente en la temperatura del aire constituyen una estimación aceptable. Entre estos métodos, los más comunes son el método de Thornthwaite, método de BlaneyCriddle, método de Hargreaves, método de Linacre y el método de Turc.

\section{Métodos basados en la radiación solar}

La mayor parte de la energía para producir la evapotranspiración proviene de la radiación, y sólo en regiones áridas y semiáridas, donde la advección juega un papel importante, la radiación no tiene una relación lineal con la evapotranspiración. La dependencia de la evapotranspiración con la radiación cambia con las condiciones climáticas y de la superficie, así como con la estación del año. Algunos métodos basados en la radiación incluyen también la temperatura.

Dentro de este grupo se encuentran el método de regresión, método Makkink, método de la Unidad Térmica Solar y el método de la radiación Solar y Térmica.

\section{Métodos combinados}

Howard Penman (1948) fue pionero en desarrollar un método de cálculo de la ET que considera tanto las fuentes de energía como el transporte turbulento del vapor de agua a partir de una superficie.

\section{Método de Penman-Monteith}

El método de Penman ha sido ampliamente utilizado para cálculos de $\mathrm{ET}_{0}$. Este método descrito en 1948 estaba pensado para el cálculo de la evaporación de superficies libres de agua, por tanto, desprecia los términos de transpiración, que no se consideran.

El método de Penman no sirve para estimar la ET en presencia de procesos de advección, por lo que frecuentemente falla para estas condiciones (Slatyer, 1970). En condiciones de fuerte advección, Rosenberg (1969) comprobó que el método de Penman subestimaba la evapotranspiración de forma considerable para un suelo desnudo. Por ello, Monteith (1963) introdujo los términos de resistencia al método de Penman. Este método ha sido el que mayor aplicación ha tenido, sobre todo en trabajos de investigación, para estimar la ET en cultivos y bosques. Posteriormente, en la década de 
los 90, Allen y colaboradores definieron un nuevo concepto de evapotranspiración de referencia. Al igual que otros modelos, está basado en el modelo de Penman, y es una modificación del método propuesto por la FAO (Doorembos y Pruitt, 1977). Este modelo calcula de forma teórica las resistencias estomática y aerodinámica de un cultivo hipotético de césped, de modo que su valor es comparable para cualquier situación geográfica.

En la actualidad para la estima de la $\mathrm{ET}_{0}$ con parámetros climáticos, el método más utilizado es el de FAO Penman-Monteith (Allen et al., 1998).

\section{Método del tanque de evaporación}

Otro método de estimación de la $\mathrm{ET}_{0}$ es el que emplea medidas directas de la evaporación de una superficie de agua, que genera un índice que integra los efectos de radiación, temperatura y humedad del aire y viento. El tanque clase A estima la evapotranspiración utilizando un coeficiente corrector $\mathrm{K}_{\mathrm{p}}$ que depende de la ubicación del tanque y de las características climáticas existentes.

\section{Métodos micrometeorológicos}

Estos métodos se basan en una combinación del balance de energía y del transporte aerodinámico del vapor de agua, utilizando diversas variables climáticas (temperatura, humedad, velocidad del viento y radiación).

\section{Método Balance de Energía y Relación de Bowen}

La evaporación precisa energía por lo que el proceso de evapotranspiración resulta limitado por la disponibilidad de energía en la superficie de vegetación. Por tanto, aplicando su principio de conservación podemos predecir la evapotranspiración.

La ecuación del balance de energía es la siguiente:

$$
\mathbf{R n}-\mathbf{G}-\lambda \mathbf{E T}-\mathbf{H}=\mathbf{0}
$$

Donde:

$\mathbf{R n}$ es la radiación neta $\left(\mathrm{W} \mathrm{m}^{-2}\right)$

G es el flujo de calor del suelo $\left(\mathrm{W} \mathrm{m}^{-2}\right)$

$\lambda \mathbf{E T}$ es el flujo de calor latente $\left(\mathrm{W} \mathrm{m}^{-2}\right)$ 
H es el calor sensible $\left(\mathrm{W} \mathrm{m}^{-2}\right)$

Se ignora la tasa neta de energía que se trasfiere horizontalmente por advección, por tanto la ecuación anterior solo es aplicable a vegetaciones homogéneas en grandes superficies.

Los términos Rn y G pueden ser estimados en función de parámetros climáticos. Sin embargo, $\mathrm{H}$ precisa de medidas de gradiente de temperatura por encima de la superficie. La estima de evapotranspiración mediante el método de transferencia de masa considera los movimientos verticales de pequeñas cantidades de aire por encima de una amplia superficie homogénea. Estos torbellinos transportan la materia (vapor de agua) y la energía (calor) desde y hacia la superficie de la evapotranspiración.

De este modo, la tasa de evapotranspiración puede ser determinada a partir de los gradientes verticales de temperatura del aire y vapor de agua mediante la relación de Bowen (Hatfield, 1989).

\section{$\underline{\text { Eddy covariance }}$}

Este método se basa en la medida de los flujos turbulentos de agua transportados verticalmente por porciones de aire, y permite conocer el flujo de calor sensible y de calor latente. La velocidad vertical de viento $\left(\mathrm{u}_{\mathrm{v}}, \mathrm{ms}^{-1}\right)$ se obtiene con un anemómetro sónico tridimensional y la densidad de vapor de agua $(\rho v)$ se mide con un higrómetro de respuesta rápida. La altura de ubicación de los sensores y la distancia entre ellos influye en la estimación de los flujos de calor sensible y calor latente (Villalobos et al., 2000).

\subsubsection{Balance hídrico}

El uso de este balance se realiza a cualquier escala. Normalmente, se miden todas las variables y se despeja la evapotranspiración según la fórmula:

$$
\mathbf{L}+\mathbf{R}-\mathbf{E T}_{\mathbf{c}}-\Delta \mathbf{H}-\mathbf{D} \pm \mathbf{E}=\mathbf{0}
$$

Donde:

L es la lluvia $\left(\mathrm{mm} \mathrm{d}^{-1}\right)$

$\mathbf{R}$ es el riego aplicado $\left(\mathrm{mm} \mathrm{d}^{-1}\right)$

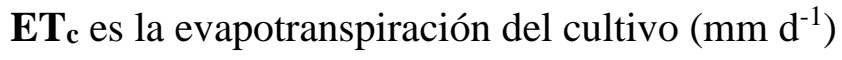


$\mathbf{\Delta H}$ es la variación de la humedad del suelo $\left(\mathrm{mm} \mathrm{d}^{-1}\right)$

D es el drenaje neto $\left(\mathrm{mm} \mathrm{d}^{-1}\right)$

E es el aporte o pérdida de agua por escorrentía superficial $\left(\mathrm{mm} \mathrm{d}^{-1}\right)$

La fiabilidad de este método depende de la precisión y veracidad de los parámetros medidos, pudiendo llegar a ser los errores de medida muy significativos. A pesar de ello, el balance de agua en el suelo ha sido muy utilizado para determinar las necesidades hídricas de cultivos arbóreos. Básicamente, se cuantifican las cantidades de agua que entran, salen o permanecen en un volumen de suelo durante un tiempo determinado (Domingo, 1994).

Con este fin, los lisímetros de pesada constituyen el método más preciso y directo de medida del consumo de agua de las plantas (Jones, 1998) pero presenta un elevado coste, laboriosidad de montaje y mantenimiento.

\subsubsection{Indicadores del estado hídrico}

La monitorización del riego constituye la técnica más efectiva para optimizar el rendimiento de los cultivos, tanto en cantidad como en calidad. Las técnicas utilizadas para la monitorización del riego pueden basarse en la estimación de la evapotranspiración, el control de la humedad del suelo o en el seguimiento del estado hídrico de la planta, como se explica a continuación.

\section{Indicadores del estado hídrico de la planta: medidas discontinuas y continuas}

La planta integra el efecto tanto de la disponibilidad de agua en el suelo como de la demanda evaporativa y, por ello, proporciona una información más adecuada para determinar el efecto de las restricciones del riego sobre su estado hídrico y para la programación del riego (Jones, 2004). Estos indicadores deben detectar lo antes posible una situación de déficit hídrico y ser representativos y robustos. Cabe diferenciar los indicadores del estado hídrico en la planta en dos grupos, según permitan la obtención de datos de forma continua o no, a fin de poder automatizar el riego. 


\section{$\underline{\text { Medidas discontinuas }}$}

Estos indicadores no permiten la automatización del riego y deben ser medidos periódicamente para obtener una evolución del estado hídrico de la planta a lo largo de un ciclo de cultivo. Entre ellos, los más usuales son los siguientes:

-Contenido relativo de agua (CRA)

Relaciona la cantidad de agua en un tejido con respecto al contenido máximo a plena turgencia. Su determinación (Barrs y Weatherley, 1962) viene dada por la fórmula:

$$
\mathrm{CRA}=\frac{\mathrm{P}_{\mathrm{F}}-\mathrm{P}_{\mathrm{S}}}{\mathrm{P}_{\mathrm{T}}-\mathrm{P}_{\mathrm{S}}}
$$

Donde:

(PF) Peso fresco de la muestra recién obtenida $(\mathrm{g})$.

(P) Peso turgente alcanzado tras sumergir la muestra en agua pura (g).

(Ps) Peso de la materia seca (g).

Según Hsiao (1990), la dificultad en su medida radica en la correcta determinación de $\mathbf{P}_{\mathbf{T}}$.

Esta técnica se considera menos rigurosa que el potencial hídrico, pero presenta la ventaja de correlacionarse mejor con el potencial de presión (principal responsable del proceso de expansión celular), que el potencial hídrico foliar (Jones, 1990).

-Potencial hídrico foliar ( $\Psi)$

Desde la aparición de la cámara de presión (Scholander et al, 1965), se ha hecho posible una lectura rápida en campo del potencial hídrico foliar.

Es conocido que el valor de potencial hídrico foliar $\left(\Psi_{\mathrm{h}}\right)$ muestra una tendencia circadiana a lo largo del día, con valores más elevados a primera y a última hora del día y menores a mediodía (Alarcón et al., 2006) ya que estos valores dependen de factores externos e internos a la propia planta. Las interacciones entre el potencial de hoja y la 
conductancia estomática, especialmente en especies isohídricas, hacen muy difícil su utilización con el fin de programar el riego.

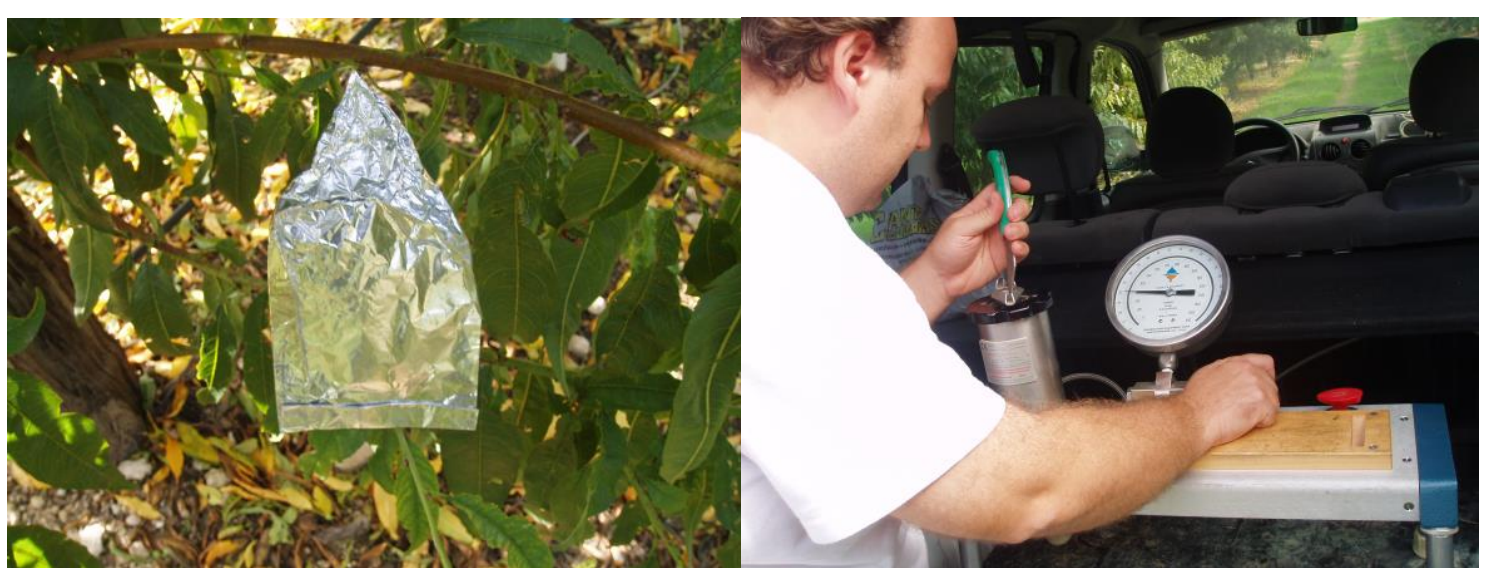

Fotografía 4.3. Hoja tapada y medición con cámara de presión. Soil Moisture Equip. Corp, model 3000, Santa Barbara, CA, USA (Fuente propia).

Por este motivo, y como alternativa, se comenzaron a utilizar indicadores menos sensibles a las condiciones ambientales como el potencial hídrico de tallo $\left(\Psi_{\mathrm{t}}\right)$. Para medirlo, se tapa una hoja cercana al tronco con una bolsa de plástico y se cubre con papel de aluminio durante al menos 2 horas antes de la medida, para impedir la transpiración (Fotografía 4.3). Al provocarse un cierre estomático, el $\Psi$ medido alcanza el equilibrio con el del xilema y, por tanto, puede ser utilizado para estimar $\Psi_{\mathrm{t}}$. Entre sus ventajas destacan su menor variabilidad, mejor correlación con el déficit de presión de vapor (DPV) y que refleja mejor la disminución de agua en el suelo que $\Psi_{\mathrm{h}}$. Diversos trabajos han puesto de manifiesto la utilidad de este indicador en diversos frutales como albaricoquero (Pérez-Sarmiento et al., 2010); granado (Intrigliolo et al., 2011); olivo (Moriana et al., 2012); uva de mesa (Gálvez et al., 2014) y melocotonero (Abrisqueta et al., 2015).

La respuesta agronómica de la planta al déficit hídrico puede representarse a través de la integral de estrés $\left(\mathrm{S}_{\Psi}\right)$, que es un índice que expresa conjuntamente la intensidad y duración del estrés (Myers, 1988), mediante la siguiente ecuación:

$$
S_{\Psi}=\left|\sum_{i=0}^{i=t}\left(\bar{\Psi}_{i, i+1}-c\right) n\right|
$$

Donde:

$\Psi_{\mathrm{i}, \mathrm{i}+1}(\mathrm{MPa})$ es el promedio de $\Psi_{\mathrm{t}}$ para cada intervalo de tiempo. 
$c(\mathrm{MPa})$ es el valor máximo de $\Psi_{\mathrm{t}}$ medido durante el estudio.

$n$ es el número de días del intervalo.

El gran inconveniente que presentan las medidas de $\Psi$ para su uso como indicador para la programación del riego es la imposibilidad de su automatización en campo.

-Intercambio Gaseoso (conductancia estomática y fotosíntesis neta)

La conductancia estomática $\left(\mathrm{g}_{\mathrm{s}}\right)$ mide el grado de apertura de los estomas, estructuras foliares que regulan el intercambio gaseoso. La transpiración se produce mayoritariamente por difusión de agua en estado de vapor a través de estos estomas aunque también existe una pérdida a través de estructuras como las lenticelas de las ramas, estomas en tallos de herbáceos o cutícula de las hojas (Kramer, 1983). La gs sigue un ritmo circadiano y está influenciada por diversos factores como la intensidad lumínica, la temperatura, el incremento de la humedad absoluta entre hoja y aire, edad de la hoja, concentración de $\mathrm{CO}_{2}$ y el potencial hídrico (Jones, 1983). Una respuesta generalizada al aumento en el DPV de la atmósfera que circunda la hoja es la disminución de la $\mathrm{g}_{\mathrm{s}}$, tal y como se ha encontrado en gran número de especies, impidiendo así tasas de transpiración excesivamente elevadas que pudiesen inducir la deshidratación del tejido (Cohen y Cohen, 1983).

Se estima que el 95\% del agua absorbida se pierde por transpiración (SánchezDíaz y Aguirreolea, 2008), de ahí la importancia de un adecuado control de estos niveles bajo una situación de déficit hídrico. Este control se produce generalmente vía estoma, esto hace que muchos autores consideren la $\mathrm{g}_{\mathrm{s}}$ como un indicador del estado hídrico de la planta. (Hsiao, 1990). El estrés hídrico tiene efecto residual sobre los estomas, de tal forma que tras la reanudación del riego se precisa un periodo de tiempo para conseguir la plena recuperación de la gs (Ruiz-Sánchez et al., 2000).

La fotosíntesis es uno de los procesos más importantes en la respuesta de las plantas ante condiciones de déficit hídrico (Azcón-Bieto et al., 1983). Implica la coordinación de distintos subprocesos tales como la absorción de $\mathrm{CO}_{2}$, la captación de luz por los complejos clorofila-proteínas, la síntesis de NADPH y la síntesis de ATP, entre otros. 


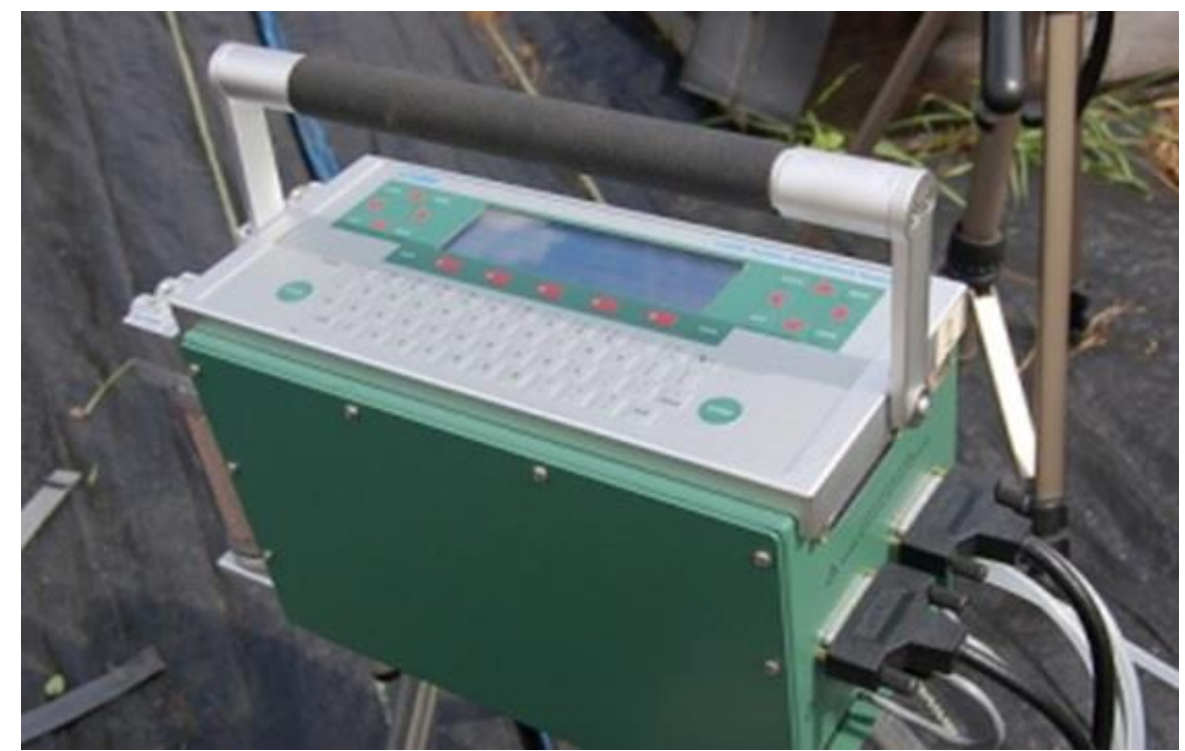

Fotografía 4.4. Sistema portátil para medida de fotosíntesis LI-6400, Li-Cor, Lincoln, NE, USA (Fuente propia).

Cuando el déficit hídrico es leve se provoca un cierre parcial de los estomas, aumentando la fotorespiración y disminuyendo la relación $\mathrm{CO}_{2} / \mathrm{O}_{2}$, lo que posibilita la rápida recuperación de la fotosíntesis tras la desaparición del estrés. (Ruiz-Sánchez et al., 2000; Medrano y Flexas, 2004). La tasa de asimilación neta de $\mathrm{CO}_{2}$ o tasa de fotosíntesis $\left(\mathrm{P}_{\mathrm{n}}\right)$ se mide con analizadores de gases por infrarrojos, algunos de ellos portátiles, que permiten su medición directa en condiciones de campo (Fotografía 4.4).

\section{-Temperatura foliar}

La temperatura en las hojas ha sido acreditada ampliamente como indicador de la respuesta de las plantas a factores ambientales adversos. La escasez de agua en el suelo hace que las plantas transpiren a una menor tasa que la demanda evaporativa de la atmósfera, lo que supone un calentamiento de la hoja ya que disminuye el efecto refrigerante de la transpiración (Kramer, 1974). Se usa desde los años 60 como indicador del estrés en la planta, siendo muy difícil su interpretación dada la gran cantidad de factores involucrados, tanto ambientales como de la planta (Gardner et al., 1992).

En 1973, Ehrler estableció un intervalo de temperaturas foliares en función de la humedad del suelo, observando una diferencia de temperatura entre la hoja y el aire que descendía $1.3{ }^{\circ} \mathrm{C}$ por cada $\mathrm{kPa}$ de aumento en el DPV. Sobre esto, Idso (1982) propusieron un índice de estrés hídrico (Crop Water Stress Index CWSI) partiendo de las relaciones entre la diferencia de temperatura entre la hoja y el aire frente al DPV, 
obteniendo líneas de base específicas para cada cultivo, que fueron mejoradas aplicando la teoría del balance de energía (Alves y Pereira, 2000). Actualmente, con la expansión de las técnicas de teledetección, el uso de la termografía infrarroja y las imágenes térmicas poseen un futuro prometedor en la monitorización del estrés hídrico de los cultivos (Jones, 2004; Sepulcre-Cantó et al., 2006).

\section{$\underline{\text { Medidas contínuas }}$}

-Cavitación xilemática

Este método se basa en la teoría por la cual el déficit hídrico puede ocasionar la rotura de la continuidad de la columna de agua dentro de los vasos xilemáticos, produciéndose así la cavitación. Cuanto mayor es el déficit hídrico mayor es el número de vasos cavitados (Tyree y Sperry, 1989). Esta interrupción en las columnas de agua libera energía en forma de emisiones acústicas que pueden ser registradas (Cruiziat et al., 2002). La frecuencia de estas pequeñas emisiones, para una especie concreta, puede ser un índice de su estado hídrico (Jones, 1989).

Hace unas décadas, para la detección de estos suaves sonidos, se recurría a su amplificación, sin embargo, los ruidos ambientales interferían seriamente. Este problema ha sido minimizado con el uso de detectores de cavitación ultrasónicos (Tyree y Dixon, 1983). El estrés hídrico no es el único factor que puede causar la cavitación en los vasos del xilema; otros factores como las heladas y los patógenos pueden influir en la aparición de la cavitación.

-Variaciones del diámetro del tronco

Las medidas de las microvariaciones de tronco y ramas proporcionan una valiosa información acerca del crecimiento y estado hídrico de la planta encontrándose directamente relacionadas con variables climáticas y con la disponibilidad de agua, resultado del desequilibrio entre transpiración y absorción de agua durante el día.

A lo largo del día, tienen lugar ciclos de contracción y expansión del diámetro del tronco alcanzando su evolución típica un valor máximo al final de la noche (MXDT), ya que la hidratación de los órganos es máxima y un valor mínimo (MNDT) que generalmente se da una vez transcurrida la transpiración máxima (Figura 4.3). 


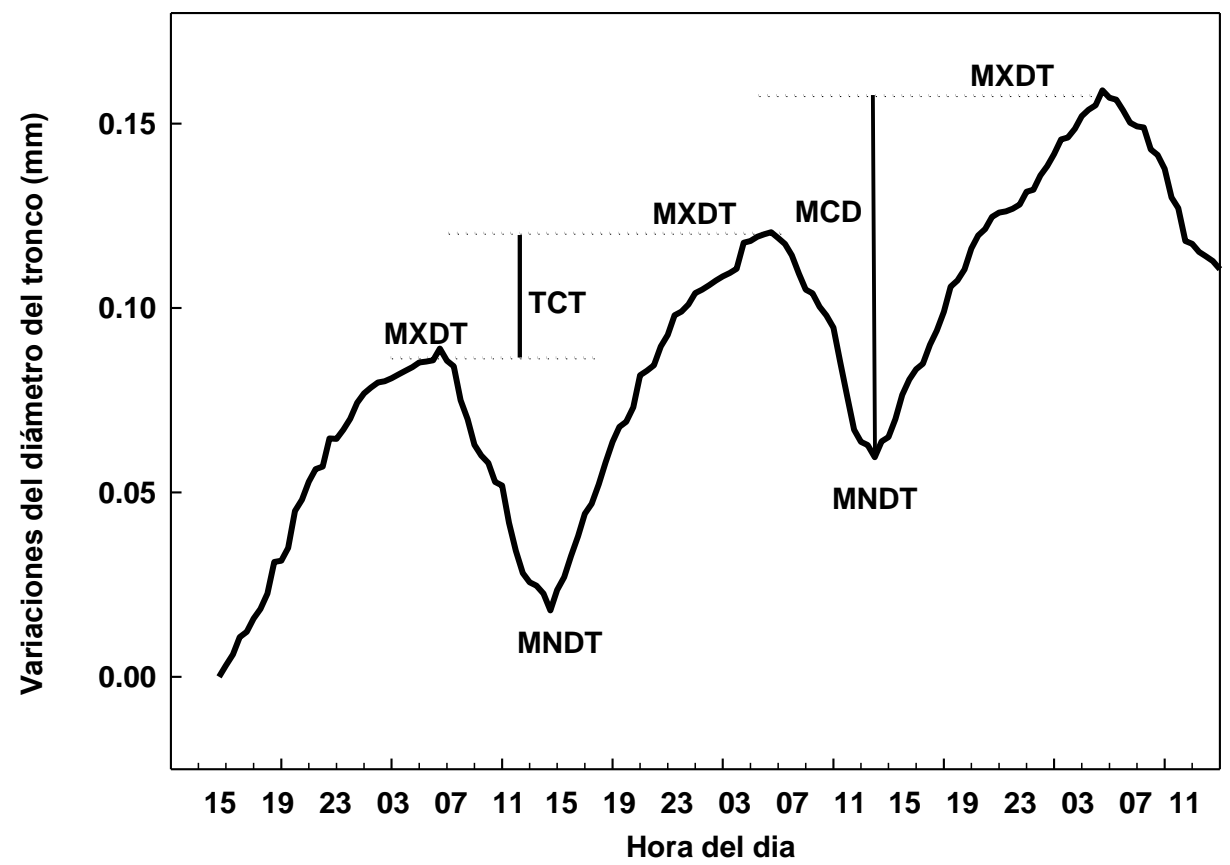

Figura 4.3. Parámetros derivados de las fluctuaciones diarias del diámetro del tronco: máximo diámetro diario de tronco (MXDT) y mínimo (MNDT), máxima contracción diaria (MCD) y la tasa de crecimiento del tronco (TCT) durante 3 días consecutivos.

La diferencia entre ambos valores es denominada máxima contracción diaria del diámetro de tronco (MCD). Dado que el xilema es prácticamente inelástico (Génard et al., 2001), los cambios en el diámetro de los troncos parecen deberse a cambios en el estado hídrico del floema (Irvine y Grace, 1997).

Por ello la magnitud de MCD va a depender de varios factores como el módulo de elasticidad y las propiedades de difusión del agua de los tejidos del floema (Génard et al., 2001), espesor del floema (Naor y Cohen, 2003; Intrigliolo y Castel, 2005) pero también del tamaño del árbol y su carga productiva (Intrigliolo y Castel, 2007). Tanto la magnitud de MCD, como la tasa de crecimiento diario del tronco (TCT), que es la diferencia de los valores de MXDT entre dos días consecutivos, representan una información valiosa sobre la intensidad del estrés hídrico alcanzado (Egea, 2008). El interés de utilizar uno u otro va a estar determinado, en gran medida, por las tasas de crecimiento del árbol. Así, en plantaciones adultas con bajas tasas de crecimiento se desaconseja el uso de TCD; en cambio, en plantaciones jóvenes ocurre lo contrario (Domingo et al., 2005; Nortes et al., 2005).

En los últimos años ha surgido un gran interés por la utilización de este indicador para gestionar el agua de riego de una forma más precisa (Fernández et al., 
2011; Moriana et al., 2013; Ballester et al., 2014). Estas medidas, usando transductores de tipo TDVL (transformador diferencial de variación lineal), pueden ser fácilmente automatizadas y utilizadas para la programación del riego (González-Altozano, 1998).

-Flujo de savia

Este método se basa en la aplicación y transporte de calor como indicador del movimiento de la savia en el tejido xilemático. Huber y Schmidt (1937) fueron los primeros en proponer el uso del pulso de calor como indicador del movimiento de la savia en el xilema, siendo demostrada su utilidad como indicador del estrés hídrico y su gran sensibilidad a los factores climáticos (Alarcón et al., 2000).

Posteriormente, se han desarrollado distintos métodos basados en la interacción entre el flujo de savia y el transporte de calor suministrado por estas técnicas. Las posibilidades de aplicación de estos métodos han aumentado paralelamente con la mejora de los sistemas informáticos ligados a la agricultura y la mejor detección de la temperatura. Las principales técnicas de medida del flujo de savia pueden agruparse en tres tipos: fuente de calor radial constante (Granier, 1985), balance de calor (Valancogne y Nasr, 1989) y pulso de calor (Nicolás et al., 2005). La elección de la técnica dependerá principalmente de la especie considerada y del tamaño del tallo o tronco.

\section{Indicadores del estado hídrico del suelo}

En la actualidad existen un buen número de instrumentos de medida del estado hídrico del suelo (Leib et al., 2003). El uso de sensores para conocer el contenido hídrico del suelo implica que el nivel de agua de éste se mantenga dentro de unos límites. El límite superior se correspondería con la capacidad de campo en el perfil y el inferior, con el umbral por debajo del cual el cultivo sufriría déficit hídrico (Campbell y Campbell, 1982). En el mercado existen distintos tipos de sensores, unos de medida del potencial mátrico del suelo y otros que miden el contenido volumétrico del agua en el suelo. 


\section{$\underline{\text { Potencial Mátrico }}$}

Este potencial representa el grado de retención del agua, debido a las interacciones con matrices sólidas o coloidales del suelo. Su valor siempre es negativo ya que la presión que lo origina se opone a la expulsión de agua del suelo; por lo tanto, cuanto más seco está un terreno, más bajo es el potencial mátrico y mayor será la presión necesaria para extraer su agua.

Los sensores que miden el potencial mátrico se basan en la medida directa de la tensión de agua en el suelo (tensiómetros) o de forma indirecta de la resistencia eléctrica del suelo (sensores de matriz granular). Estos sensores tienen restricciones técnicas que limitan su rango de trabajo y precisión en suelos en fase de desecación rápida (Thompson et al., 2006), a pesar de ello continúan usándose con éxito (Pedrero et al., 2013).

La disponibilidad de agua para los cultivos depende más del potencial mátrico del suelo que de su contenido en agua por lo que, en principio, el potencial mátrico debe ser mejor indicador del estado hídrico del suelo con relación al desarrollo de las plantas (Hillel, 1973). A pesar de ello, el hecho de que el estado hídrico de la planta sea controlado por diferentes procesos biológicos hace que su validez como indicador único quede en entredicho ya que este solo podrá reflejar el estado hídrico parcialmente.

\section{Contenido volumétrico de agua en el suelo}

Los sensores de medida del contenido volumétrico de agua en el suelo incluyen la sonda de neutrones y la sonda de reflectometría en el dominio del tiempo (TDR) (Phene et al., 1990).

Los límites para el inicio del riego con estos sensores barajan la combinación de dos conceptos: por un lado el agua disponible o útil, cantidad de agua entre la capacidad de campo y punto de marchitez y, por otro lado, el nivel de agotamiento permisible, el cual se refiere al contenido de humedad de un suelo por debajo del cual la absorción radicular consume energía. Por ello es conveniente mantener los niveles de humedad del suelo entre valores de capacidad de campo y de agotamiento permisible, es decir en valores de agua fácilmente disponible (Allen et al., 1998; Fares y Polyakov, 2006). 


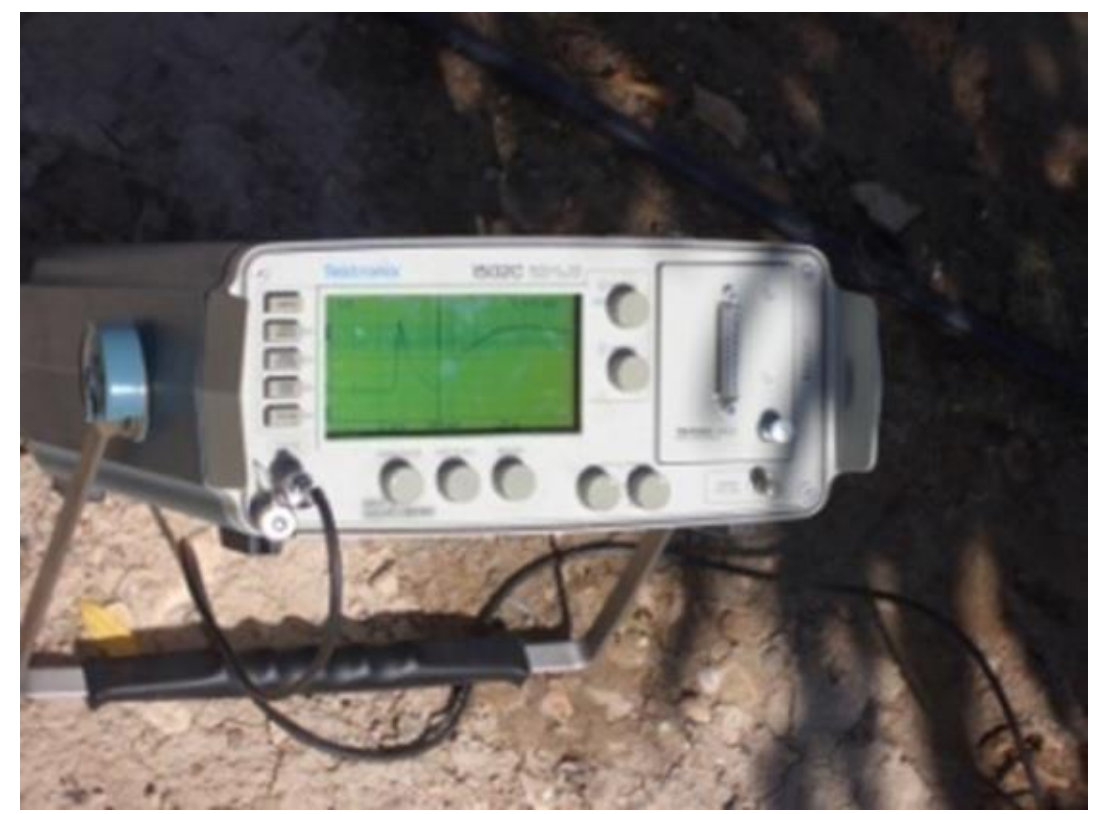

Fotografía 4.5. Equipo Time Domain Reflectometry (TDR). Model 1502C, Tektronix Inc., OR. (Fuente propia).

La metodología de TDR se basa en la medida del tiempo de propagación de un pulso electromagnético a lo largo de una línea de transmisión (sonda) insertada en el suelo, (Cole, 1977). Su rango de medida oscila de 0,065 a $0,5 \mathrm{~cm}^{3} \mathrm{~cm}^{-3}$. Estas sondas tienen como principal ventaja que no necesitan calibración y permiten medir con precisión el contenido volumétrico de agua en el suelo (Fotografía 4.5).

Pueden tomarse lecturas en continuo y puntuales y, dentro de unos límites, no se ven afectadas por la salinidad. Se trata de una medida integradora de la humedad de toda la longitud de la sonda y, para las principales clases de suelo no se requiere una calibración, aunque en el caso de ciertos sustratos, de suelos arcillosos o con elevado contenido de materia orgánica, puede ser necesaria.

Otro equipo que se puede utilizar para la medida del contenido volumétrico de agua en el suelo es la sonda de neutrones (Fotografía 4.6). Se trata de un aparato radioactivo que permite estimar la humedad o densidad de los suelos. Para ello se dispone un tubo de acceso de aluminio introducido en el suelo para que, deslizando la fuente radioactiva en él, estima la humedad del suelo a la cota requerida.

Esta sonda consta de un cilindro metálico sellado de 3 a $5 \mathrm{~cm}$ de diámetro y de 20 a $30 \mathrm{~cm}$ de longitud el cual contiene una fuente de ${ }^{241} \mathrm{Am}-{ }^{9} \mathrm{Be}$. El americio bombardea con partículas $\alpha$ los núcleos de Berilio que emiten un haz de neutrones con alta energía cinética (neutrones rápidos) que, al chocar con partículas con un similar 
peso molecular, como los átomos de hidrógeno del agua, pierden su energía ralentizándose y pasando a denominarse neutrones lentos rebotando hacia su lugar de partida donde, con la ayuda de un contador Geiger Müller, podemos estimar la cantidad de agua contenida en el suelo. El uso de estos aparatos se ha limitado a los centros de investigación debido a su riesgo radioactivo y la complejidad de su mantenimiento (Gear et al., 1977).

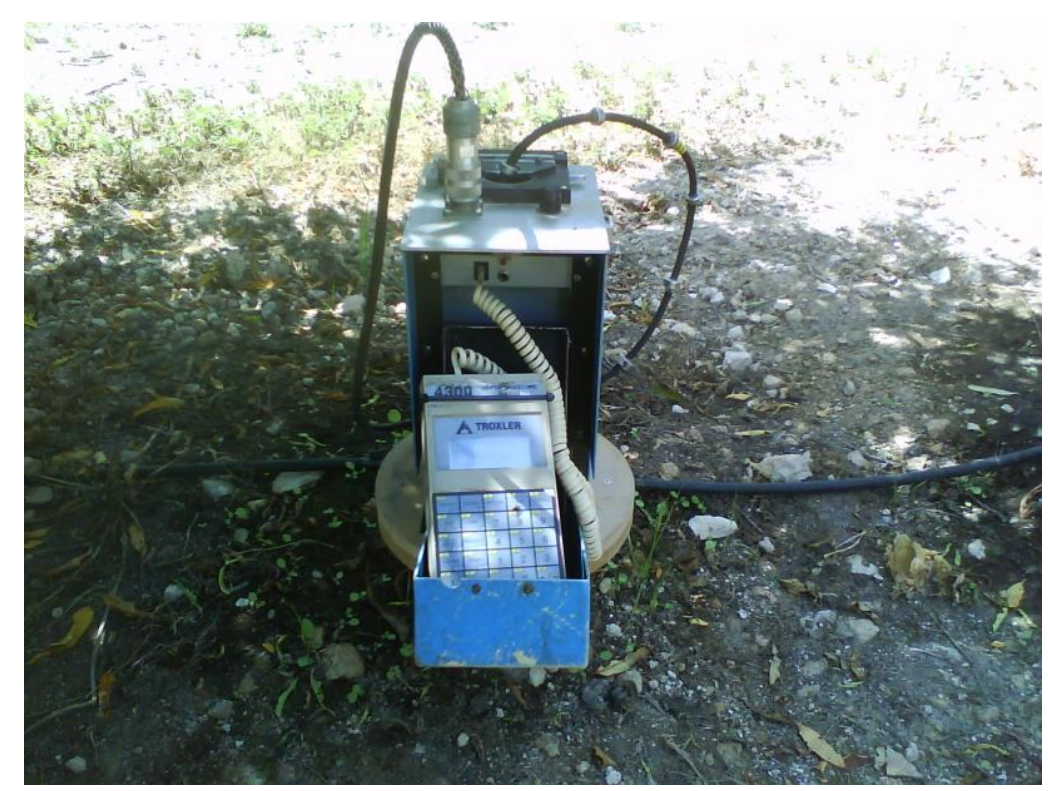

Fotografía 4.6. Sonda de neutrones. Model 4300, Troxler Electronic Laboratories Inc., NC (Fuente propia).

\subsection{Concepto de Riego Deficitario Controlado (RDC)}

Actualmente están aumentando los desequilibrios entre la oferta y la demanda de recursos hídricos, más acentuados debido al aumento exponencial de la población y a una cada vez mayor industrialización, lo que provoca cierta competencia por este bien. Los desequilibrios climáticos están trayendo como consecuencia una distribución de las precipitaciones menos uniforme, hecho que se agrava en regiones áridas y semiáridas del planeta. Ante estas limitaciones actuales se deben emprender acciones dirigidas al incremento de los recursos, adquiriendo prioridad las acciones orientadas al aumento de la eficiencia y el ahorro hídrico. A este respecto no existen dudas de la conveniencia de emprender acciones que tiendan a mejorar la gestión del agua, tales como la necesaria mejora de las infraestructuras hidráulicas, y la flexibilización de la distribución del recurso en función de la demanda de los cultivos.

Dentro de esta línea de aumento de la eficiencia y ahorro hídrico se encuentra el denominado riego deficitario controlado (RDC), basado en reducir los aportes hídricos 
en aquellos períodos fenológicos considerados no críticos para la producción y calidad de la cosecha, cubriendo plenamente la demanda de la planta durante el resto del ciclo del cultivo. Esta técnica surge a partir de los primeros trabajos de Chalmers et al. (1981), en los que se pretendía controlar el exceso de vigor en plantaciones frutales de melocotonero, sin que la producción se viera afectada y que sirvió para poner de manifiesto la posibilidad de inducir estrés hídrico en el período de máximo crecimiento vegetativo sin comprometer la productividad (Chalmers et al., 1981; Mitchell y Chalmers, 1982), consiguiendo ahorros de agua importantes con reducciones de riego en las fases I y II de crecimiento del fruto. El RDC tiene un enfoque más fisiológico que el riego deficitario $(\mathrm{RD})$ ya que contempla tanto la fenología del cultivo, como su capacidad para resistir situaciones de déficit hídrico. Con esta estrategia de riego se puede maximizar la producción por unidad de agua aplicada (Domingo et al., 2005).

Hasta la fecha se han realizado numerosos ensayos en distintas especies de frutales, entre ellos destacan: melocotonero (Conejero et al., 2011; Vera et al., 2013), albaricoquero (Pérez-Pastor et al., 2009; Pérez-Sarmiento et al., 2010), cítricos (Ballester et al., 2013; Mounzer et al., 2013), granado (Intrigliolo et al., 2013; Laribi et al., 2013), almendro (Egea et al., 2013) entre otras. En la mayoria de los estudios, las estrategias de RDC en frutales suponen un importante ahorro de agua, aumentando la eficiencia en el uso de la misma (Egea et al., 2010; Hueso y Cuevas, 2010), una disminución del crecimiento vegetativo (Pérez-Pastor et al., 2009), lo cual se traduce en un ahorro de costes de producción (poda), y una producción final que no se ve afectada por la reducción de aportes los hídricos, ni en cantidad, ni en calidad, llegando incluso esta última a mejorar bajo estrategias de RDC (Pérez-Sarmiento et al., 2010; Alcobendas et al., 2013; Laribi et al., 2013).

\subsection{Establecimiento de una estrategia de RDC}

Para establecer una estrategia de RDC deben tenerse en cuenta aquellos factores que puedan condicionar su viabilidad. Entre otros, adquiere una especial importancia el conocimiento de los períodos críticos del cultivo, la coincidencia entre el crecimiento vegetativo y reproductivo, el sistema de riego, el clima, las características del suelo, la resistencia a la sequía, etc. (Torrecillas et al., 1996). Además, resulta imprescindible determinar aquellos períodos fenológicos en los que el desarrollo de un estrés puede afectar, de forma considerable, la producción y/o la calidad de la cosecha, así como la 
intensidad y duración de los mismos para un correcto manejo del RDC (Torrecillas et al., 1996). Asimismo, es necesario conocer la separación natural de los períodos durante los cuales los tejidos y los órganos de los frutales crecen activamente, ya que posibilita el inhibir el crecimiento de un órgano, un tejido o proceso, sin afectar seriamente a otros. Además, algunos frutos como el melocotón y los cítricos, al recuperarse de un déficit hídrico temporal experimentan un crecimiento compensatorio que les permite alcanzar un tamaño similar al de los frutos que no han experimentado déficit (PérezSarmiento et al., 2010).

\subsubsection{Periodos críticos del cultivo}

Son aquellos periodos en los que el estrés hídrico puede condicionar de forma considerable la producción y/o calidad de la cosecha. Por ello, la programación del riego en los periodos no críticos es esencial en la aplicación de estrategias de RDC, debiéndose determinar la dosis de agua a aplicar para seleccionar las condiciones más adecuadas en las que realizar el riego.

Sin embargo, resulta difícil indicar los periodos críticos concretos ya que son distintos para cada cultivo. Por ejemplo, en melocotonero el déficit hídrico durante la tercera fase del crecimiento del fruto o fase de rápido crecimiento resulta más trascendente que en otras fases fenológicas (Mitchell y Chalmers, 1982; Goldhamer et al., 2002), por lo que debe evitarse la aplicación de esta estrategia de RDC en este periodo. Sin embargo, Intrigliolo y Castel (2005) señalaron que en otros cultivos como el ciruelo los efectos de la supresión del riego son más dependientes de la duración y del nivel de coincidencia con períodos de alta demanda evaporativa, que del momento en el que tiene lugar su supresión, encontrando una alta sensibilidad del cultivo durante el período de endurecimiento del hueso, contrariamente a lo observado en melocotonero.

En albaricoquero, según Pérez-Pastor et al. (2009), el período más crítico al déficit hídrico es la segunda fase de rápido crecimiento del fruto (fase III), junto al de postcosecha inicial (Ruiz-Sánchez et al., 1999), período en el que se registran altos consumos de agua. La supresión del riego durante este período de postcosecha inicial afecta a la diferenciación floral, lo que provoca un desarrollo tardío de las yemas florales (Brown, 1953), disminución de su número y alteración de las mismas. 
No sólo deben tenerse en cuenta los momentos de alta incidencia del déficit hídrico, sino también la magnitud y la duración de éste, ya que a largo plazo podría tener una serie de efectos no deseables, siendo conveniente realizar, para cada cultivo y situación, los pertinentes ensayos que nos permitan conocer las respuestas que experimentan los diferentes cultivos de cara a la adopción de este tipo de métodos, por las repercusiones económicas que ello conlleva y el importante ahorro de recursos hídricos que puede suponer (Sánchez-Blanco y Torrecillas, 1995).

\subsubsection{Crecimiento vegetativo $\mathrm{v}$ de fruto}

Resulta esencial, ante la aplicación de estrategias de RDC, conocer en cada cultivo cuándo tiene lugar tanto el crecimiento vegetativo como el productivo, y además qué nivel de coincidencia existe entre uno y otro. Una clara separación entre ambos puede definir la idoneidad de este tipo de estrategias de RDC para un determinado cultivo. Durante el periodo de brotación y desarrollo de ramos, si reducimos los aportes hídricos podemos limitar este proceso para atender plenamente las demandas hídricas de la planta durante el desarrollo del fruto, sin limitar el tamaño final del mismo (PérezSarmiento et al., 2010; López et al., 2010; Alcobendas et al., 2013).

\subsubsection{Sistema de riego}

El sistema de riego ideal para llevar a cabo estrategias de RDC es el riego localizado, ya que nos permite aplicar volúmenes reducidos de agua en el suelo y controlar más fácilmente la cantidad de agua aplicada. Entre las ventajas del riego localizado se encuentran su mejor aprovechamiento del agua, reducción de la mano de obra, sobre todo porque disminuyen las malas hierbas al no humedecer la totalidad del suelo, y la posibilidad de automatización. Sin embargo, no todo son ventajas, el alto coste de instalación y mantenimiento son desventajas a tener en cuenta.

\subsubsection{Clima}

El clima es otro factor que influye en la aplicación de estrategias de RDC en el sentido de que éstas suelen ir encaminadas, entre otras razones, a programar situaciones de estrés hídrico en determinados momentos del ciclo de un cultivo, por lo que la presencia de lluvias puede ser un aspecto negativo en la consecución de este fin. En climas donde sean frecuentes las lluvias resulta conveniente suprimir los aportes hídricos en aquellos períodos donde sea necesario limitar el desarrollo vegetativo, de tal 
manera que se permita asegurar un tiempo suficiente de déficit hídrico e inducir el efecto deseado.

\subsubsection{Características del suelo}

Algunos frutos, como el melocotonero y los cítricos, son susceptibles de experimentar crecimientos al reanudar el riego tras un déficit hídrico. Además los cítricos, durante el estrés hídrico, acumulan materia seca que va a facilitar el crecimiento del fruto tras la reanudación del riego. Esto indica la necesidad de facilitar la aparición del déficit hídrico como recuperación. De ahí que Chalmers (1990) indicara que suelos poco profundos con baja capacidad de retención de agua resultan convenientes para su aplicación, al conseguirse un rápido agotamiento y recarga de los niveles de humedad del suelo, además de volúmenes reducidos de suelo humectado, que permitirían una alta concentración de raíces. Otro aspecto a considerar bajo estas circunstancias es controlar el crecimiento radicular durante los períodos de déficit hídrico, ya que se ha observado que al ralentizar el desarrollo radicular, se ve limitado el crecimiento vegetativo y se favorece el del fruto, ya que las sustancias elaboradas en la fotosíntesis se canalizan hacia los frutos.

\subsubsection{Resistencia a la sequía}

Otro factor importante a tener en cuenta es la capacidad de los cultivos para adaptarse a las condiciones de déficit hídrico. La resistencia a la sequía de un cultivo hace referencia a su capacidad para crecer satisfactoriamente en zonas con déficit hídrico. Según Lange et al (1971), la escasez de agua juega un importante papel al determinar la distribución y abundancia de muchas especies vegetales, de ahí que el conocimiento de los mecanismos de resistencia a la sequía tenga una gran importancia tanto desde un punto de vista tanto ecológico como agrícola.

Las modificaciones que tienen lugar en la estructura y función de las plantas para aumentar la probabilidad de sobrevivir y reproducirse en un ambiente determinado se llama adaptación. La influencia de los mecanismos adaptativos sobre la productividad del cultivo fue estudiada por Turner en 1986, comprobando que sólo aquellos mecanismos que favorecen el escape a la sequía, el mantenimiento de la entrada de agua y el mantenimiento de la presión de turgencia, no reducen la fotosíntesis, el crecimiento ni el rendimiento del cultivo. 
Se distinguen tres mecanismos de adaptación de las plantas a la sequía (Blum, 1988; Ceccarelli, 1989):

1. Mecanismos de escape de la sequía: capacidad de las plantas para completar su ciclo antes de que el déficit hídrico en el suelo y en la planta se desarrollen. Para este fin, la planta cumple una determinada fase fenológica de forma rápida o, en todo caso, adapta su desarrollo a las condiciones dominantes en ese momento.

2. Mecanismos de aplazamiento o evitación de la deshidratación: capacidad de las plantas para mantener un potencial hídrico relativamente alto en condiciones de estrés hídrico, atmosférico o del suelo, mediante el cierre estomático y el ajuste osmótico.

3. Mecanismos de tolerancia a la deshidratación: (i) capacidad de las plantas para reducir la actividad química del agua; (ii) concentrar solutos y macromoléculas y (iii) producir modificaciones en las membranas celulares. 


\subsection{Bibliografía}

Abrisqueta, I., Conejero, W., Valdés-Vela, M., Vera, J., Ortuño, M.F., Ruiz-Sánchez, M.C., 2015. Stem water potential estimation of drip-irrigated early-maturing peach trees under Mediterranean conditions. Computers and Electronics in Agriculture 114, 7-13.

Alarcón J.J, Domingo R, Green S, Sánchez-Blanco M.J, Rodríguez P, Torrecillas A., 2000. Sap flow as an indicator of transpiration and the water status of young apricot trees. Plant and Soil 227, 77-85.

Alarcón, J.J., Torrecillas, A., Sánchez-Blanco, M.J., Abrisqueta, J.M., Vera, J., Pedrero, F., Magaña, I., García-Orellana, Y., Ortuño, M.F., Nicolás, E., Conejero, W., Mounzer, O., Ruiz-Sánchez, M.C. 2006 Estrategias de riego deficitario en melocotonero temprano. Vida Rural 225, 28-32.

Alcobendas, R., Mirás-Ávalos, J.M., Alarcón, J.J., Nicolás, E., 2013. Effects of irrigation and fruit position on size, colour, firmness and sugar contents of fruits in a mid-late maturing peach cultivar. Scientia Horticulturae 164, 340-347.

Allen, R.G., Pereira, L.S., Raes, D., Smith, M., 1998. Crop evapotranspiration. Paper 56. FAO of the United Nations. Roma (Italia). 300 p.

Alves, I., Pereira, L.S., 2000. Modeling surface resistance from climatic variables?. Agricultural Water Management 42, 371-385.

Azcón-Bieto, J. Lambers, H., Day, D.A., 1983. The effect of photosynthesis and carbohydrate status on respiration rates and the involvement of the alternative path in leaf respiration. Plant Physiology 72, 598-603.

Ballester, C., Buesa, I., Bonet, L., Intrigliolo, D.S., 2014. Usefulness of stem dendrometers as continuous indicator of loquat trees water status. Agricultural Water Management 142, 110-114.

Ballester, C., Castel, J., Intrigliolo, D.S., Castel, J.R., 2013. Response of Navel Lane Late citrus trees to regulated deficit irrigation: yield, components and fruit composition. Irrigation Science 31, 333-341.

Barrs, H.D., Weatherley, P.E., 1962. A re-examination of the relative turgidity technique for estimating water deficits in leaves. Australian Journal of Biological Sciences 15, 413-28.

Blum, A., 1988. Plant Breeding for Stress Environments. CRC Press, Florida, p.223.

Brown, D.S., 1953. The effects of irrigation on flower bud development and fruiting in the apricot. Proceedings of the American Society for Horticultural Science 61, 119-124.

CARM, 2009. Estadística agraria de Murcia. Consejería de Agricultura y Agua de la Región de Murcia. www.carm.es 
Campbell, G.S., Campbell, M.D., 1982. Irrigation scheduling using soil moisturemeasurements. En: Advances in irrigation (D. Hillel Ed.) Academic Press, Inc., New York, Vol 1: 25-42.

Ceccarelli, S., 1989. Wide adaptation: How wide? Euphytica 40, 197-205.

Chalmers, D.J., Mitchell, P.D., Van Heek, L., 1981. Control of peach tree growth and productivity by regulated water supply, tree density and summer pruning. Journal of American Society for Horticultural Science 106, 307- 312.

Chalmers, D.J., 1990. Control del crecimiento de la planta por la regulación de los déficits de agua y la limitación de la zona de humidificación. Fruticultura 5, 369375.

Cohen, M., 1994. Funcionamiento hidrico y produccion frutal del nogal en zonas semiaridas: Aplication al manejo del riego. Tesis Doctoral. Universitat de Lleida (Udl), Lleida, 298 p.

Cohen, S., Cohen, Y., 1983. Field studies of leaf conductance response to environmental variables in citrus. Journal of Applied Ecology 20, 561-70.

Cole, R., 1977. Time Domain Reflectometry. Annual Review of Physical Chemistry Vol. 28, 283-300.

Conejero, W., Mellisho, C.D., Ortuño, M.F., Moriana, A., Moreno, F., Torrecillas, A., 2011. Using trunk diametre sensors for regulated deficit irrigation scheduling in early maturing peach trees. Environmental and Experimental Botany 71, 409415.

Cruiziat, P., Cochard, H., Ameglio, T., 2002. Hydraulic architecture of trees: main concepts and results. Annals of Forest Sciences 59, 723-752.

Domingo, R., 1994. Respuesta del limonero Fino al riego deficitario controlado. Aspectos fisiológicos. Tesis Doctoral. Universidad de Murcia. 237 pp.

Domingo, R., Nortes, P.A., Egea, G., Torres, R., Pérez Pastor, A., 2005. Utilización de sensores en planta para la programación del riego y uso eficiente del agua en la agricultura. Vida Rural 217, 42-46.

Doorenbos, J., Pruitt, W.O., 1977. Crop water requirements. Irrigation and Drainage. Paper No24 (rev.). FAO, Rome, 144p.

Egea, G., 2008. Characterization and modelling of the agronomic and physiologic responses of almond trees to water deficit. $\mathrm{PhD}$ thesis (shortened version). Universidad Politécnica de Cartagena, Spain. 90 pp.

Egea, G., Nortes, P.A., González-Real, M.M., Baille, A., Domingo, R., 2010. Agronomic response and water productivity of almond trees under contrasted deficit irrigation regimes. Agricultural Water Management 97, 171-181.

Egea, G., Nortes, P.A., Domingo, R., Baille, A., Pérez-Pastor, A., González-Real, M.M., 2013. Almond agronomic response to long-term déficit irrigation applied since orchard establishment. Irrigation Science 31, 445- 454. 
Ehrler, W.L., 1973. Cotton leaf temperature as related to soil water depletion and meteorological factors. Agronomy Journal 65, 404-409.

FAO, 2015. Sitio web AQUASTAT

Fares, A., Polyakov, V., 2006. Advances in crop water management using capacitance sensors. Advances in Agronomy 90, 43-77

Fernández, J.E., Moreno, F., Martín-Palomo, M.J., Cuevas, M.V., Torres-Ruiz, J.M., Moriana, A., 2011. Combining sap flow and trunk diameter measurements to assess water needs in mature olive orchards. Environmental and Experimental Botany $72,330-338$.

Gálvez, R., Callejas, R., Reginato, G., Peppi, M.C., 2014. Irrigation schedule on table grapes by stem water potential and vapor pressure deficit allows to optimize water use. Ciência e Técnica Vitivinícola 29(2), 60-70.

Gardner, B.R., Nielsen, D.C., Shock, C.C., 1992. Infrared thermometry and the crop water stress index: I. History, Theory and Baselines. Journal of Production Agriculture 5, 462-466.

Gear, R.D., Dansfield, A.S., Campbell, M.D., 1977. Irrigation scheduling with neutron probe. Journal of the Irrigation and Drainage Division. (ASCE) 103(IR3), 291298.

Génard, M., Fishman, S., Vercambre, G., Huguet, J.G., Bussi, C., Besset, J., Habib, R., 2001. A biophysical analysis of stem and root diameter variations in woody plants. Plant Physiology 126 (1), 188-202.

Goldhamer, D.A., Salinas, M., Crisosto, C., Day, K.R., Soler, M., Moriana, A., 2002. Effects of regulated deficit irrigation and partial root zone drying on late harvest peach tree performance. Acta Horticulturae 592, 343-350.

González-Altozano, P., 1998. Riego deficitario controlado en Clementina de Nules: relaciones hídricas y respuesta agronómica. Tesis doctoral. Universidad Politécnica de Valencia. 173 pp.

Granier, A., 1985. Une nouvelle methode pour la mesure des flux de seve dans le tronc des arbres. Annales des Sciences Forestieres 42, 193-200.

Hatfield, J.L., 1989. Aerodinamics properties of partial canopies. Agricultural and forest Meteorology 46, 15-22.

Hillel, D., 1990. Role of irrigation in agricultural systems. En: Irrigation ofagricultural crops. Stewart, BA. y Nielsen, DR., (ed.). Agronomy. Wisconsin, USA: p 5-31.

Hillel, D., Guron. Y., 1973. Relation between evapotranspiration rate and maize yield. Water Resources Research 9, 743-748.

Hsiao, T.C., 1990. Measurements of plant water status. En: Irrigation of Agricultural Crops. Steward, BA. and Nielsen, DR., (ed.). Madison, Wis.: American Society of Agronomy. p 243-279. 
Huber, B., Schmidt, E., 1937. A compensation method for thermoelectric measurement of slow sap flow. Berichte der Deutschen Botanischen Gesellschaft 55, 514-529.

Hueso, J.J., Cuevas, J., 2010. Ten consecutive years of regulated déficit irrigation probe the sustainability and profitability of this water saving strategy in loquat. Agricultural Water Management 97, 645-650.

Idso, S.B., 1982. Non-water-stressed baselines: a key to measuring and interpreting plant water stress. Agricultural Meteorology 27, 59-70.

Intrigliolo, D.S., Castel, J.R., 2005. Effects of regulated deficit irrigation on growth and yield of young Japanese plum trees. Journal of Horticultural Science \& Biotechnology 80, 177-182.

Intrigliolo, D.S., Castel, J.R., 2007. Crop load affects maximum daily trunk shrinkage of plum trees. Tree Physiology 27, 89-96.

Intrigliolo, D.S., Puerto, H., Bonet, L., Alarcón, J.J., Nicolás, E., Bartual, J., 2011. Usefulness of trunk diameter variations as a continuous water stress indicators of pomegranate (Punica granatum) trees. Agricultural Water Management 98, $1462-1468$.

Intrigliolo, D.S., Bonet, L., Nortes, P.A., Puerto, H., Nicolás, E., Bartual, J., 2013. Pomegranate trees performance under sustained and regulated deficit irrigation. Irrigation Science 31, 959-970.

Irvine, J., Grace, J., 1997. Continuous measurement of water tensions in the xylem of trees based on the elastic properties of wood. Planta 202, 455-461.

Jones, H.G., 1983. Estimation of an effective soil water potential at the root surface of transpiring plants. Plant, Cell and Environment 6, 671-674.

Jones, H.G., 1989. Water stress and stem conductivity. En: Environmental Stress in Plants. (J.H. Cherry, ed.). Springer-Verlag. Berlín. 19, 17-24.

Jones, H.G., 1990. Physiological aspects of the control of water status in horticultural crops. Horticultural Science 25, 19-26.

Jones, H.G., 1998. Stomatal control of photosynthesis and transpiration. Journal of Experimental Botany 49 (Special Issue), 387-398

Jones, H., 2004. Irrigation scheduling: advantages and pitfalls of plant-based methods. Journal of Experimental Botany 55, 2427-2436.

Kramer P.J., 1974. Fifty years of progress in water relations research. Plant Physiology $54,463-471$

Kramer, P.J., 1983. Water Relations of Plants. Academic Press. New York. 489 pp.

Lange, O.L., Lösch, R., Schulze, E.D., Kappen, L. 1971. Responses of stomata to changes in humidity. Planta 100, 76-86.

Laribi, A.I., Palou, L., Intrigliolo, D.S., Nortes, P.A., Rojas-Argudo., C., Taberner, V., Bartual, J., Pérez-Gago, M.B., 2013. Effect of sustained and regulated deficit 
irrigation on fruit quality of pomegranate cv. "Mollar Elche" at harvest and during cold storage. Agricultural Water Management 125, 61-70.

Leib B.G., Jabro J.D., Mathews G.R., 2003. Field evaluation and performance comparison of soil moisture sensors. Soil Science 168, 396-408

Libro Blanco del Agua en España. MMA. 2008

López, G., Behboudian, M.H., Vallverdu, X., Mata, M., Girona, J., Marsal, J. 2010. Mitigation of severe water stress by fruit thinning in 'O'Henry'peach: implications for fruit quality. Scientia Horticulturae 125, 294-300.

MAGRAMA, 2015a. Boletin mensual de Estadistica. 2015. Enero

MAGRAMA, 2015b. Anuario de estadística Avance 2014. Ministerio de Agricultura, Alimentación y Medio Ambiente.

Medrano, H., Flexas, J., 2004. Respuesta de las plantas al estrés hídrico. En: Reigosa M., N. Pedrol y A. Sánchez (eds.). Ecofisiología vegetal: una ciencia de síntesis. Editorial Thomson, Madrid. pp. 253-286.

Mitchell, P.K., Chalmers, D.J., 1982. The effects of reduced water supply on peach tree growth and yields. Journal of the American Society for Horticultural Science $107,853-856$.

Monteith, J.L., 1963. Gas exchange in plant communities. In Environmental Control of Plant Growth, ed. L. T.Evans, pp. 95- 112. New York: Academic. 449 pp.

Moriana, A., Corell, M., Girón, I.F., Conejero, W., Morales, D., Torrecillas, A., Moreno, F., 2013. Regulated deficit irrigation based on threshold values of trunk diameter fluctuation indicators in table olive trees. Scientia Horticulturae 164, $102-111$.

Moriana, A, Pérez-López, D, Prieto, M.H, Ramírez-Santa-Pau, M, Pérez-Rodriguez, J.M., 2012. Midday stem water potential as a useful tool for estimating irrigation requirements in olive trees. Agricultural Water Management 112, 43-54

Mounzer, O., Pedrero-Salcedo, F., Nortes, P.A., Bayona, J.M., Nicolás, E., Alarcón, J.J., 2013. Transient soil salinity under the combined effect of reclaimed water and regulated deficit drip irrigation of Mandarin trees. Agricultural Water Management 120, 23-29.

Myers, B.J., 1988. Water stress integral-a link between short-term stress and long-term growth. Tree Physiology 4, 315-323.

Naor, A., Cohen, S., 2003. Sensitivity and variability of maximum trunk shrinkage, midday stem water potential, and transpiration rate in response to withholding irrigation from field-grown apple trees. Horticultural Science 38, 547-551.

Nicolás, E., Torrecillas, A., Dell'Amico, J., Alarcon, J., 2005. The effect of short-term flooding on the sap flow, gas exchange and hydraulic conductivity of young apricot trees. Trees-Structure and Function 19, 51-57. 
Nortes, P.A., Perez-Pastor, A., Egea, G., Conejero, W., Domingo, R., 2005. Comparison of changes in stem diameter and water potential values for detecting water stress in young almond trees. Agricultural Water Management 77, 296-307.

Pedrero, F., Mounzer, O., Alarcón, J.J., Bayona, J.M., Nicolás, E., 2013. The viability of irrigating mandarin trees with saline reclaimed water in a semi-arid Mediterranean region: a preliminary assessment. Irrigation Science 31, 759-768

Penman, H.L., 1948. Natural evaporation from open water, bare soil and grass. Proceedings of the Royal Society of London, Series A 193, 120-148.

Pérez-Pastor, A., Domingo, R., Torrecillas, A., Ruiz-Sánchez, M.C., 2009. Response of apricot trees to deficit irrigation strategies. Irrigation Science 27, 231-242.

Pérez-Sarmiento, F., Alcobendas, R., Mounzer, O., Alarcón, J.J., Nicolás, E., 2010. Effects of regulated deficit irrigation on physiology and fruit quality in apricot trees. Spanish Journal of Agricultural Research 8, 86-94.

Phene, C.J., Reginato, R.J., Itier, B., Tanner, B.R., 1990. Sensing irrigation needs. Pp 207-261. In: G.J. Hoffman, T.A. Howell, and K.H. Solomon (eds). Management of Farm Irrigation Systems, American Society of Agricultural Engineers. St. Joseph, MI.

Rosenberg, N.J., 1969. Seasonal patterns of evapotranspiration by irrigated alfalfa in the central Great Plains. Agronomy Journal 61, 879-886.

Ruiz-Sánchez, M.C., Egea, J., Galego, R., Torrecillas, A., 1999. Floral biology of 'Búlida' apricot trees subjected to postharvest drought stress. Annals of Applied Biology 135, 523-528.

Ruiz-Sánchez, M.C., Torrecillas, A., Pérez-Pastor, A., Domingo, R., 2000. Regulated deficit irrigation in apricot trees. Acta Horticulturae 537, 759-766.

Sánchez-Blanco, M.J., Torrecillas, A., 1995. Aspectos relacionados con la utilización de estrategias de riego deficitario controlado en cultivos leñosos. En: Riego Deficitario Controlado. Fundamentos y Aplicaciones. Colección Cuadernos Value 1. Mundi Prensa/Unión Europea. pp. 43-63.

Sánchez-Díaz, M., Aguirreolea, J., 2008. Transpiración y control estomático. En: Azcón-Bieto, J.; Talón, M. (Eds.). Fundamentos de fisiología vegetal. McGrawHill Interamericana de España (Madrid). p.41-56.

Scholander, P.F., Hammel, H.T., Bradstreet, E.D., Hemingsen, E.A., 1965. Sap pressure in vascular plants. Science 148, 339-346.

Sepulcre-Cantó, G., Zarco-Tejada, P.J., Jiménez-Muñoz, J.C., Sobrino, J.A., de Miguel, E., Villalobos, F.J., 2006. Detection of water stress in an olive orchard with thermal remote sensing imagery. Agricultural and Forest Meteorology 136, 3144.

Slatyer, R.O., 1970. Comparative photosynthesis, growth and transpiration of two species of Atriplex. Planta 93, 175-189. 
Thompson, R.B., Gallardo, M., Agüera, T., Valdez, L.C., Fernández, M.D., 2006. Evaluation of the Watermark sensor for use with drip irrigated vegetable crops. Irrigation Science 24, 185-202.

Torrecillas, A., Alarcon, J.J., Domingo, R., Planes, J. y Sanchez Blanco, M.J., 1996. Strategies for drought resistance in leaves of two almond cultivars. Plant Science $118,135-143$.

Turner, N.C., 1986. Crop water deficits: a decade of progress. Advances in Agronomy $39,1-51$.

Tyree, M.T., Dixon, M.A., 1983. Cavitation events in Thuja occidentalis L. Plant Physiology 72, 1094-1099.

Tyree M.T, Sperry J.S., 1989. Vulnerability of xylem to cavitation and embolism. Annual Review of Plant Physiology and Plant Molecular Biology 40, 19-38.

Valancogne, C., Nasr, Z., 1989. Measuring sap flow in the stem of small trees by a heat balance method. Horticultural Science (24), 383-385.

Vera, J., Abrisqueta, I., Abrisqueta, J.M., Ruiz-Sánchez, M.C., 2013. Effect of deficit irrigation in early-maturing peach tree performance. Irrigation Science 31, 747757.

Villalobos, F.J., Orgaz, F., Testi, L., Fereres, E., 2000. Measurement and modeling of evapotranspiration of olive (Olea europaea L.) orchards. European Journal of Agronomy 13, 155-163. 
REFERENCE VALUES OF MAXIMUM DAILY TRUNK SHRINKAGE FOR IRRIGATION SCHEDULING IN MIDLATE MATURING PEACH TREES 



\title{
5. REFERENCE VALUES OF MAXIMUM DAILY TRUNK SHRINKAGE FOR IRRIGATION SCHEDULING IN MID-LATE MATURING PEACH TREES
}

\begin{abstract}
Precise irrigation is essential in arid and semi-arid areas where water is scarce, such as the South-East of Spain. Therefore, finding a precise and reliable water stress indicator which could be automatically controlled is of paramount importance. Maximum daily trunk shrinkage (MDS) showed a great potential for commercial irrigation scheduling. However, MDS records may be difficult to interpret and reference baselines could be useful for this interpretation. In the current study, measurements of midday stem water potential $\left(\Psi_{\mathrm{s}}\right)$ and MDS were taken over a 3-year period in peach trees [Prunus persica (L.) Batsch cv. 'Catherine'] in order to obtain reference baselines for irrigation scheduling. Plants were daily irrigated above their water requirements $(\sim 120 \%)$ in order to obtain non-limiting soil water conditions. Climate parameters and MDS were recorded automatically, whereas midday stem water potential was assessed in the field every 3 days. The relationships between MDS and climate parameters such as $\mathrm{ET}_{0}$, daily maximum and midday air temperatures $\left(\mathrm{T}_{\max }\right.$ and $\mathrm{T}_{\text {mid }}$, respectively), maximum and midday air vapour pressure deficit (VPD $\mathrm{Vax}_{\max }$ and $\mathrm{VPD}_{\text {mid }}$, respectively) were determined. The results indicate that MDS records were better related to $\mathrm{T}_{\text {mid }}$ and $\mathrm{T}_{\max }$ than to other meteorological variables. The relationship between MDS and $\Psi_{\mathrm{s}}$ differed from year to year, although similar equations were found among seasons for the post-harvest period. However, these relationships varied greatly with the conditions of each year, tree phenological stage and crop load. Therefore, the growers must be aware of these factors when using MDS values for irrigation scheduling.
\end{abstract}

Keywords: Maximum daily trunk shrinkage; Baselines; Stem water potential; Prunus persica; Irrigation scheduling.

\subsection{Introduction}

In the world context, peach tree [Prunus persica (L.) Bastch] is one of the most extended fruit crops, the second most important fruit species by production after apple. Its great acceptance by consumers is due to its diverse uses and fresh consumption. Spain is the second largest peach producer in the world. Peach tree plantations are located in the Mediterranean area, being Murcia (SE Spain) the third main peach 
production area in the country after Aragon and Catalonia, with a cultivated area of 10,713 ha and a production of $171526 \mathrm{t}$ per year, which represents $21 \%$ of the Spanish total production (MAGRAMA, 2015).

Currently, the pressure on water resources is increased by the competition for water between agriculture, industry and population, leading to an urgent need to develop new techniques of irrigation scheduling that allow increasing crop water productivity in irrigated agriculture, which accounts for up to $70-80 \%$ of the total water consumption in arid and semi-arid zones (Fereres and Soriano, 2007).

The evaluation of the crop water status, by means of direct measurements in the plants constitutes a valuable tool for irrigation management given its dynamic nature, which is closely related to the climatic and soil conditions, as well as with crop productivity (Goldhamer et al., 2003; Remorini and Massai, 2003). Among these, several indicators have been studied such as stem water potential $\left(\Psi_{\mathrm{s}}\right)$ (discontinuous measurement) and trunk diameter fluctuations (TDF) (continuous measurement) (Conejero et al., 2011). In this sense, $\Psi_{\mathrm{s}}$ is traditionally the most commonly used parameter to estimate the plant water status due to its reliability, low variability and a moderately good prediction of yield response to water stress (Naor, 2006; Shackel et al., 1997). However, since its measurement cannot be easily automated, finding water stress indicators that can be continuously recorded has been of great interest for researchers in the last years.

Within these, the maximum daily trunk shrinkage (MDS) has been proposed as a suitable tool for precise irrigation scheduling (Goldhamer and Fereres, 2001) because its relatively uncomplicated monitoring procedure providing continuous and automatic information of plant water status in contrast to discontinuous measures such as the $\Psi_{\mathrm{s}}$, where regular visits to the field make it an impractical and costly method. The MDS measurements have demonstrated their sensitiveness to changes in tree water status in many stone-fruit species as apricot trees (Nicolás et al., 2005), extra-early maturing peach trees (Conejero et al., 2007) and in nut-fruit species as almond (Egea et al., 2009). However, the fact that MDS is the result not only of the soil water available to the plant but also of the evaporative demand of the atmosphere means that using absolute MDS values for irrigation scheduling is unsuitable. 
Consequently, it is better to use the concept of MDS signal intensity, normalizing the MDS values with respect to values under non-limiting soil water conditions (MDS actual value/MDS reference value) in order to avoid the effect of climatic conditions (Fernández and Cuevas, 2010).

Usually, the reference values are obtained by irrigating trees above their water needs or by means of the elaboration of reference equations (baseline relationships) in which the values of the plant-based indicator are correlated with a climatic variable closely related to the evaporative demand (Fernández and Cuevas, 2010). Moreno et al. (2006) showed that MDS in olive trees reflected changes in the evaporative demand variables, even though the MDS behaviour was best correlated with both midday vapour pressure deficit $\left(\mathrm{VPD}_{\mathrm{mid}}\right)$ and air temperature at midday $\left(\mathrm{T}_{\mathrm{mid}}\right)$. In addition, Pagán et al. (2012) reported the suitability of MDS to establish reference baselines for irrigation scheduling in mandarin trees due to its linear response and good correlation with temperature.

They found that MDS was best correlated with $\mathrm{T}_{\text {mid }}$; however, they recommended the use of average $\left(\mathrm{T}_{\mathrm{m}}\right)$ or maximum air temperature $\left(\mathrm{T}_{\max }\right)$ due to easiness in their measurement. De la Rosa et al. (2013) showed the suitability of MDS for establishing reference lines to be used in early nectarine trees for irrigation

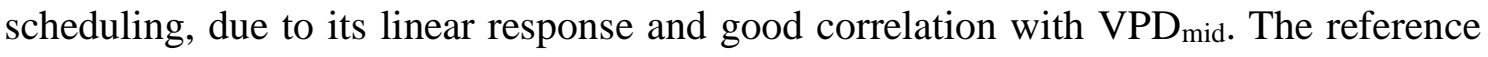
lines that they established were stable over three years, and were not crop-load dependent, at least in the range studied. Respect to peach trees, Conejero et al. (2011) measured $\Psi_{\mathrm{s}}$ and MDS over a 4-year period in early maturing peach trees [Prunus persica (L.) Batsch cv. 'Flordastar'] and found that the effects of environmental conditions on daily MDS values were dependent on crop load, indicating that specific MDS reference equations should be developed as a function of crop load for irrigation scheduling. These findings mean that MDS measurements may need adjustments before they can be used for irrigation scheduling.

Therefore, it is necessary to test the baselines stability, both in the short (through the season) and the long-term (over a few years), in order to prove that it is a good and reproducible method for irrigation scheduling. 
In this context, the objective of this study was to obtain MDS reference equations in mid-late maturing peach trees, based on appropriate climate variables and $\Psi_{\mathrm{s}}$, for their use in irrigation scheduling.

\subsection{Materials and Methods}

\section{Site description and plant material}

The experiment was conducted over three consecutive years (2008-2010) in a 0.5 ha plot of a commercial orchard located in Mula valley, Murcia, SE Spain $\left(37^{\circ} 55^{\prime} \mathrm{N}\right.$, $1^{\circ} 25^{\prime} \mathrm{W}, 360 \mathrm{~m}$ above sea level). The soil at the site is calcareous, stony, with a sandyloam texture, low organic matter content and its available water capacity is $0.25 \mathrm{~m}^{3} \mathrm{~m}^{-3}$, approximately. The climate of the region is semiarid Mediterranean with hot and dry summers and low rainfall; annual evaporation calculated from reference crop evapotranspiration $\left(\mathrm{ET}_{0}\right)$ and rainfall during the growing season were, respectively, 792 and $254 \mathrm{~mm}$ in the first year of experiments (2008), 613 and $304 \mathrm{~mm}$ in 2009 and 784 and $188 \mathrm{~mm}$ in 2010.

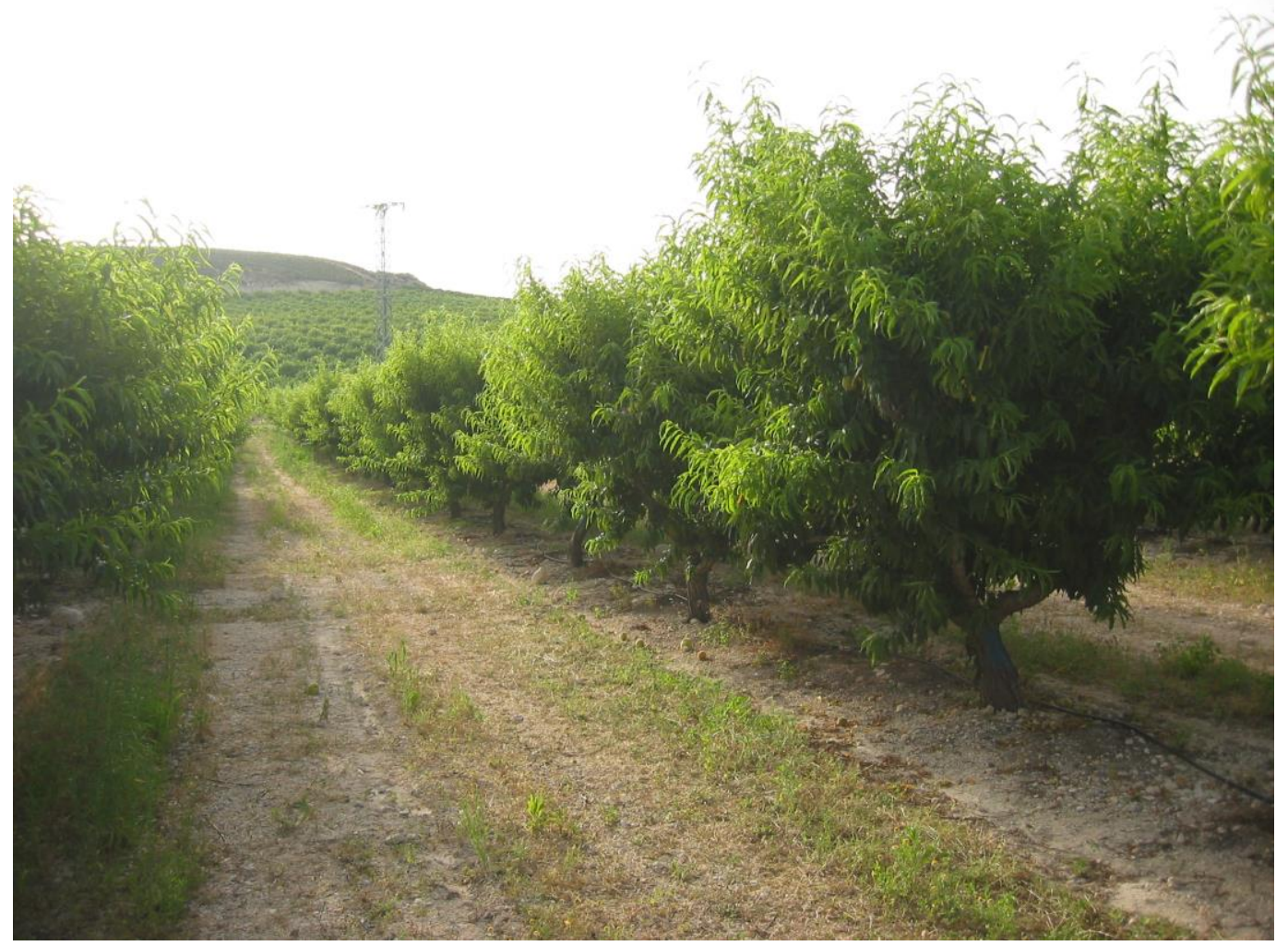

Picture 5.1. View of the experimental site (Own source). 
Plant material consisted of peach trees [Prunus persica (L.) Batsch cv. 'Catherine'] planted in 1999 and grafted on GF-677 rootstock. Spacings were 4 m x 6 $\mathrm{m}$. Hand thinning was used to space fruitlets $20 \mathrm{~cm}$ along the fruit bearing shoots in order to achieve a commercial crop load. Pest control was that commonly used by the growers and all treatments received the same fertilization $\left(\mathrm{N}-\mathrm{P}_{2} \mathrm{O}_{5}-\mathrm{K}_{2} \mathrm{O}\right)$, applied through the drip irrigation system $\left(275-125-200 \mathrm{~kg} \mathrm{ha}^{-1}\right.$ year $\left.^{-1}\right)$ over the study period.

\section{Irrigation treatment and experimental design}

Irrigation water was supplied through a drip irrigation system, one pipeline for each row, with three emitters per tree (delivering $4 \mathrm{~L} \mathrm{~h}^{-1}$ each). The irrigation water was considered of good quality with a very low electrical conductivity $\left(0.6 \mathrm{dS} \mathrm{m}^{-1}\right)$.

Crop irrigation requirements were scheduled weekly according to daily $\mathrm{ET}_{0}$, calculated using the Penman-Monteith equation (Allen et al., 1998), and a local crop factor based on the time of the year and used in this region at commercial level. Monthly average values of $\mathrm{K}_{\mathrm{c}}$ employed from April to October were 0.73, 0.83, 0.94, $0.96,0.39,0.39$ and 0.39 respectively. A total of 64 trees were used in this study. The experiment was laid out in completely randomized blocks with 4 replications (16 trees each). The four central trees of each replicate were used for measurements, and the other 12 trees acted as guard.

Trees were irrigated above their water requirements $\left(\sim 120 \% \mathrm{ET}_{\mathrm{c}}\right)$ throughout the experimental period in order to obtain non limiting soil water conditions.

Irrigation was automatically controlled by a head unit programmer and the amounts of water applied for each irrigation treatment were measured with in-line flowmeters placed in each experimental plot.

\section{Field and laboratory determinations}

\section{Climate data}

Climate data were recorded at an automatic weather station placed within the experimental orchard. Data on air temperature (maximum, minimum and average), solar radiation, air relative humidity, rainfall and wind speed $2.5 \mathrm{~m}$ above the soil surface, were collected every $15 \mathrm{~min}$. These data were used for calculating $\mathrm{ET}_{0}$ and crop water 
requirements. In addition, data of midday air temperature and air vapour pressure deficit (VPD) were extracted from these records.

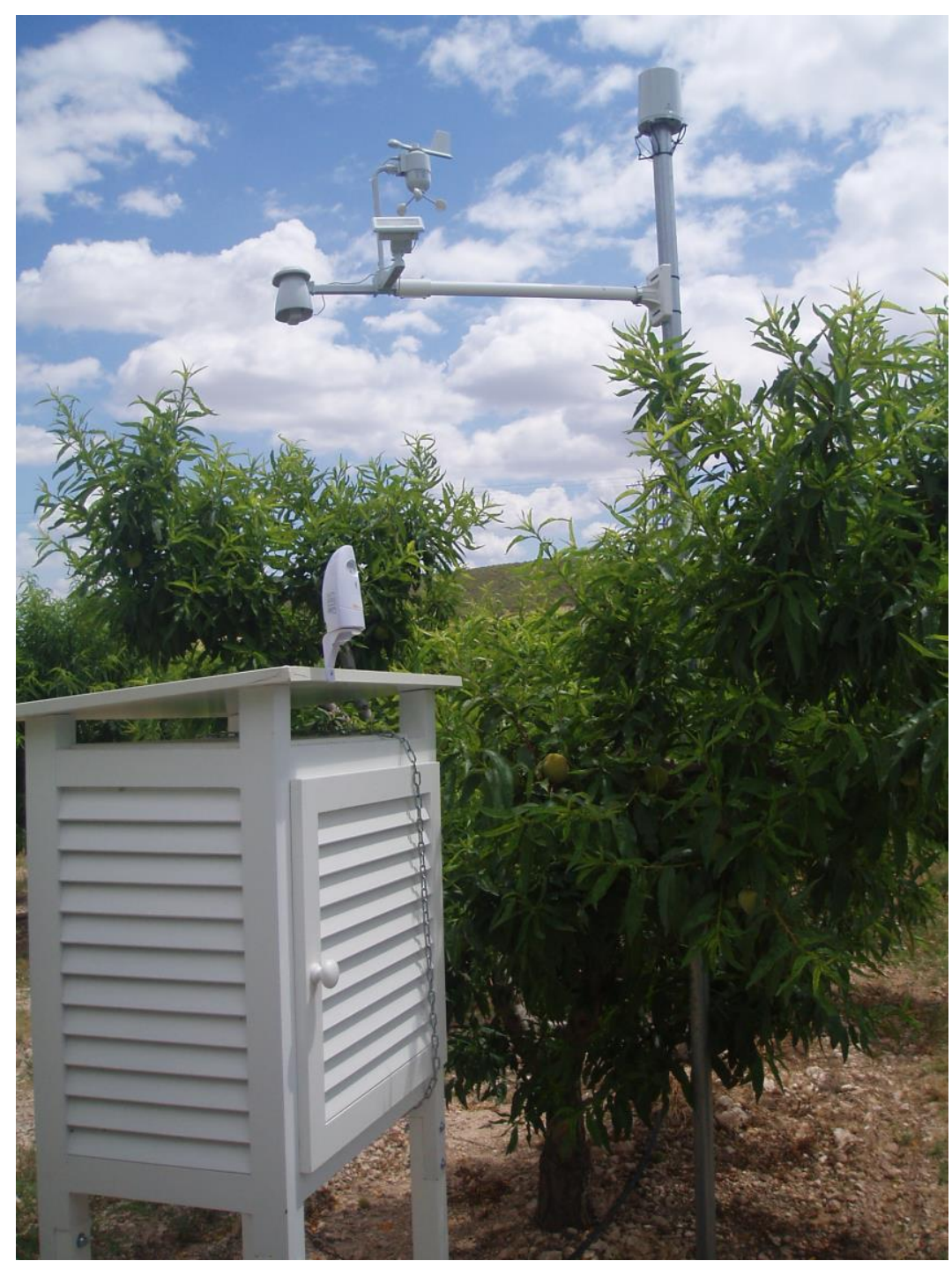

Picture 5.2. Automatic weather station placed within the experimental orchard (Own source).

\section{Stem water potential}

Midday stem water potential $\left(\Psi_{\mathrm{s}}\right)$ was measured every 3 days using a pressure chamber (Soil Moisture Equip. Corp, model 3000, Santa Barbara, CA, USA) following the procedures described by Turner (1981). Measurements were performed on mature leaves (one per tree) from the north face of the trunk, in the four central trees of each experimental plot. Leaves were enclosed in plastic bags covered with aluminum foil at least two hours prior to the measurements, which were carried out between 12:00 and 13:00 h solar time. 


\section{Trunk diameter fluctuations}

The micrometric trunk diameter fluctuations (TDF) were measured throughout the experimental period in four trees (one tree per experimental plot), using linear variable displacement transducers (LVDT) (Solartron Metrology, Bognor Regis, UK, model DF $\pm 2.5 \mathrm{~mm}$, accuracy $\pm 10 \mu \mathrm{m}$,) attached to the trunk, with a special bracket made of Invar, an alloy of $\mathrm{Ni}$ and Fe with a thermal expansion coefficient close to zero (Katerji et al., 1994), and aluminium. Sensors were placed on the north side and were covered with silver thermoprotected foil to prevent heating and wetting of the device. In order to eliminate measurement noise, old bark was carefully eliminated with a sharpened knife. Then, TDF measurements included only the contributions of the phloem and xylem. Measurements were taken every $30 \mathrm{~s}$ and one datalogger and a multiplexor (model CR1000, Campbell Scientific, Inc., Logan, USA) were programmed to report 15 minute-means. Maximum daily trunk shrinkage (MDS) was calculated daily as the difference between maximum and minimum daily trunk diameter (Goldhamer and Fereres, 2001).

\section{Yield and crop load}

Fruits were individually harvested on July in two or three commercial picks, depending on market demands in the four central trees of each experimental plot. The number of fruits per tree was obtained by counting the fruits in the four inner trees of each plot.

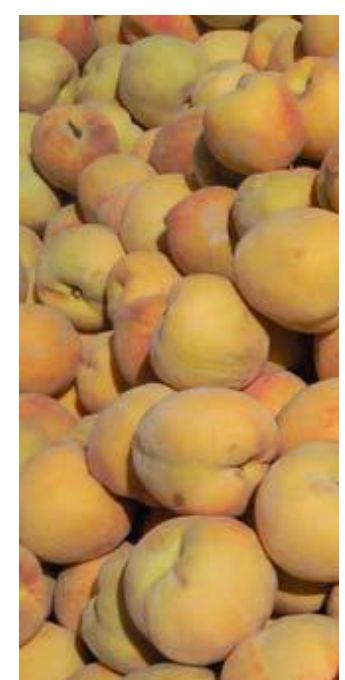

Picture 5.3. Harvested "Catherine" peaches (Own source). 
Trunk cross-sectional area (TCSA) and canopy volume

Trunk diameter was measured annually in all trees, during the dormancy stage with a sliding caliper, $0.20 \mathrm{~m}$ above the soil surface, and used to estimate trunk crosssectional area (TCSA). Linear and transverse lengths and heights of the canopy were measured annually in all trees of treatment to estimate the canopy volume (Westwood, 1988).

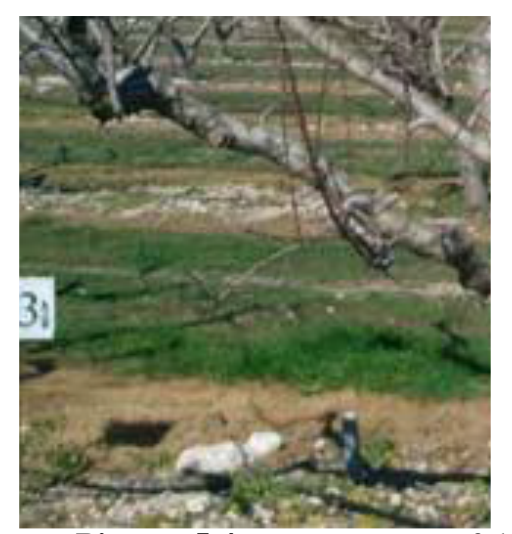

Picture 5.4. Measurement of the tree canopy (Own source).

Statistical analysis

Statistical analysis of tree characteristics among years was performed as a weighted analysis of variance (ANOVA; statistical software IBM SPSS Statistics v. 21 for Windows). The Shapiro-Wilk test was used to evaluate the normality of the data. Relationships between MDS measurements and the other variables ( $\Psi_{\mathrm{s}}$ and climate variables) were assessed through Pearson's $r$ correlation coefficient and linear regression techniques.

The differences in slopes and intercepts of the fitted lines among seasons were assessed through an analysis of covariance (ANCOVA). Unless otherwise stated, the significance level was $p \leq 0.05$. 


\subsection{Results}

Climatic variables showed a similar dynamics for the different years studied (Figure 5.1). However, rainfall was different in 2008 than on the other two growing seasons studied; higher rainfall amounts were registered in springtime in 2008, whereas rainfall peaks at the end of the summer were observed in 2009 and 2010. Potential evapotranspiration over the growing season was very similar in 2008 and 2009, while greater $\mathrm{ET}_{0}$ values were recorded in 2010, mainly until the end of August.

Daily mean VPD values were very similar among years, whereas the midday values of this variable differed greatly, being higher in 2009 (Figure 5.1). Mean air temperatures were slightly higher in 2009 and 2010 than in 2008; however, midday air temperature showed a similar trend between years, with slightly greater values in 2009 (Figure 5.1).

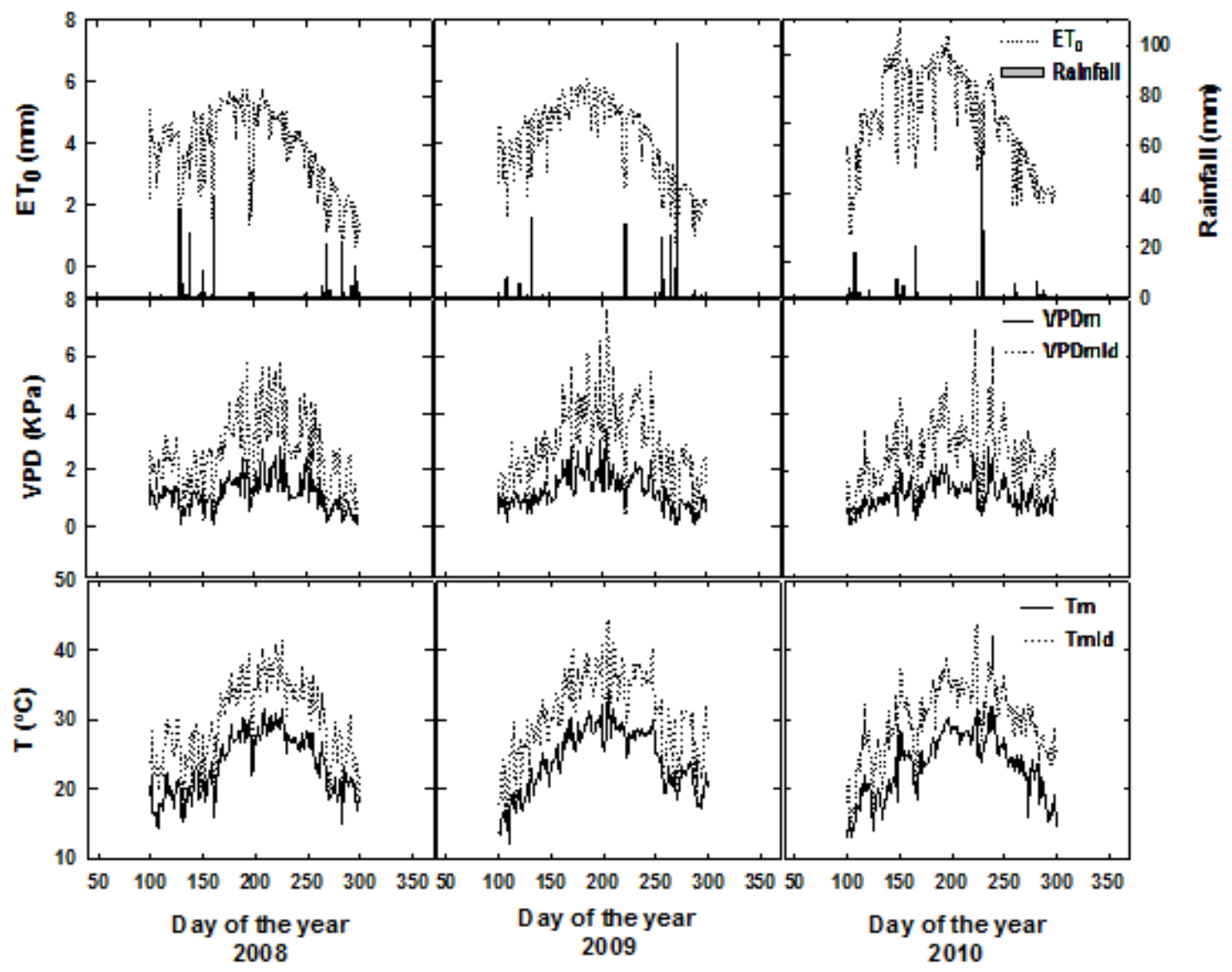

Figure 5.1. Evolution of different climate parameters over the three years studied (2008-2010). Potential evapotranspiration $\left(\mathrm{ET}_{0}\right)$, mean daily temperature $\left(\mathrm{T}_{\mathrm{m}}\right)$, midday temperature $\left(\mathrm{T}_{\mathrm{mid}}\right)$, mean air vapour pressure deficit $\left(\mathrm{VPD}_{\mathrm{m}}\right)$ and midday air vapour pressure deficit $\left(\mathrm{VPD}_{\mathrm{mid}}\right)$.

Tree characteristics differed from year to year (Table 5.1). As the trees grew, trunk diameter and TCSA increased from the first to the last year of experiments. 
However, canopy volume was similar on the last two years of studies, being greater than that measured at the beginning of the experiment. In contrast, crop load and yield were, respectively, 4 and 2.5 times greater in 2009 than in 2008 and 2010 (Table 5.1).

The dynamics of $\Psi_{\mathrm{s}}$ over the growing season was different from year to year (Figure 2). The most negative values of $\Psi_{\mathrm{s}}$ were measured in 2008 (with a minimum of $-1.3 \mathrm{MPa}$ at post-harvest) and the least negative ones in 2010. The greatest differences among years for this water stress indicator were observed at post-harvest. In 2008, $\Psi_{\text {s }}$ values ranged from -0.35 to $-1.3 \mathrm{MPa}$, whereas in 2009 and 2010 they varied between -0.4 and $-1 \mathrm{MPa}$ for the fruit growth and post-harvest periods.

Similarly, MDS values over the growing season were slightly different from one year to the next (Figure 5.2). In 2008, MDS showed the lowest values, ranging from 30 to $260 \mu \mathrm{m}$, for the three growing seasons studied. In 2009, MDS varied between 40 and $290 \mu \mathrm{m}$; whereas in 2010, it oscillated between 20 and $300 \mu \mathrm{m}$ (Figure 5.2). The highest MDS values were observed at the end of fruit growth in the two first years and at early post-harvest in the last year of the experiment.

Table 5.1. Characteristics of the studied trees over the three growing seasons studied (2008-2010).

\begin{tabular}{|c|c|c|c|c|c|}
\hline Year & $\begin{array}{c}\text { Trunk diameter } \\
\text { (cm) }\end{array}$ & $\begin{array}{c}\text { Canopy volume } \\
\left(\mathbf{m}^{\mathbf{3}}\right)\end{array}$ & $\begin{array}{l}\text { TCSA } \\
\left(\mathrm{cm}^{2}\right)\end{array}$ & $\begin{array}{c}\text { Crop load } \\
\left.\text { (Fruits tree }{ }^{-1}\right)\end{array}$ & $\begin{array}{r}\text { Yield } \\
\left(\mathrm{kg} \mathrm{tree}^{-1}\right)\end{array}$ \\
\hline 2008 & $13.93 \mathrm{c}$ & $7.93 \mathrm{~b}$ & $155.2 \mathrm{c}$ & $150.31 \mathrm{~b}$ & 24.73 b \\
\hline 2009 & $15.00 \mathrm{~b}$ & $8.48 \mathrm{a}$ & $179.4 \mathrm{~b}$ & 630.17 a & $61.47 \mathrm{a}$ \\
\hline 2010 & $15.80 \mathrm{a}$ & 8.39 ab & $199.2 \mathrm{a}$ & $152.67 \mathrm{~b}$ & $26.01 \mathrm{~b}$ \\
\hline 2008-2010 & 14.91 & 8.27 & 177.9 & 311.05 & 37.40 \\
\hline
\end{tabular}

Different letters in the column indicate significant differences among years at $p<0.05$.

According to these observations, the relationships between $\Psi_{\mathrm{s}}$ and MDS differed among years and between fruit growth and post-harvest stages (Figure 5.2). In 2008, $\Psi_{\mathrm{s}}$ and MDS were linearly related during fruit growth, but this relationship was not maintained at post-harvest. In contrast, the relationships between these two plant-based indicators in 2010 were very similar for fruit growth and post-harvest. The intermediate case was observed in 2009 (Figure 5.2).

The relationships between MDS and climate parameters such as daily maximum air temperature $\left(\mathrm{T}_{\max }\right)$, midday air temperature $\left(\mathrm{T}_{\mathrm{mid}}\right)$, maximum air vapour pressure 
deficit $\left(\mathrm{VPD}_{\max }\right)$ and midday $\mathrm{VPD}\left(\mathrm{VPD}_{\text {mid }}\right)$ and $\mathrm{ET}_{0}$ during the three years of the experiment showed the best fit in a lineal form (Figures 5.3 and 5.4).

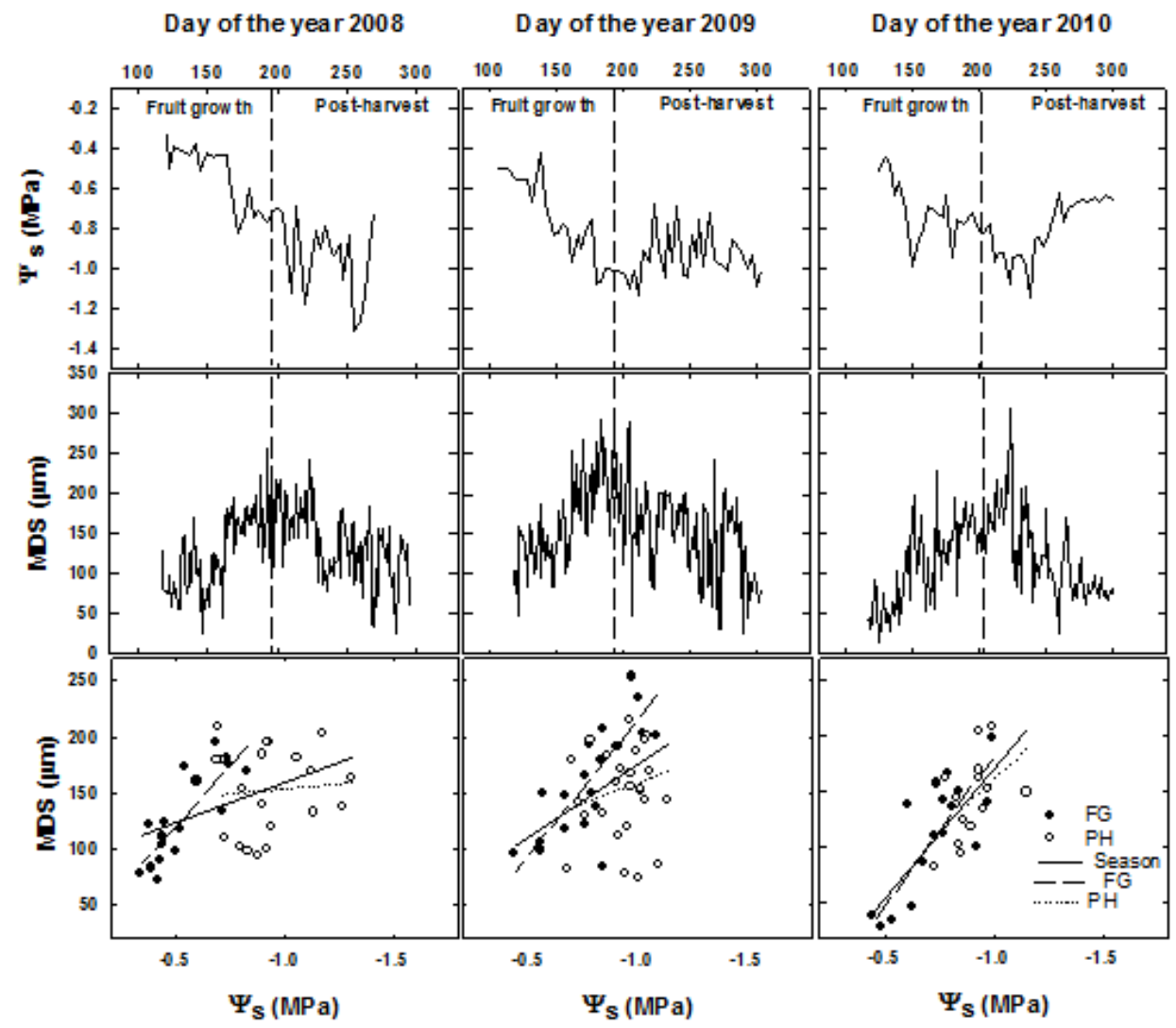

Figure 5.2. Midday stem water potential $\left(\Psi_{\mathrm{s}}\right)$ and maximum daily trunk diameter shrinkage (MDS) dynamics over the three growing seasons studied (2008-2010). The relationships between both parameters are depicted for each season. FG and PH indicate fruit-growth and post-harvest stages, respectively.

All the relationships were significant, although the degree of correlation varied between parameters and years (Table 5.2). The coefficients of determination were rather low, especially for $\mathrm{ET}_{0}\left(\mathrm{r}^{2}\right.$ between 0.21 and 0.54 , depending on the year). The highest $r^{2}$ was observed for $T_{\text {mid }}$ in $2010\left(r^{2}=0.69\right)$. When data for the three seasons were pooled together, the rank order for the determination coefficients was as follows: $\mathrm{T}_{\max }=$ $\mathrm{T}_{\text {mid }}>\mathrm{VPD}_{\max }=\mathrm{VPD}_{\min }>\mathrm{ET}_{0}($ Table 5.2).

ANCOVA results proved that the slopes of the relationships between MDS and $\mathrm{ET}_{0}$ were only significantly different for the fruit growth stage, whereas the intercepts were significantly different among seasons in all cases. In the case of the relationships between MDS and $\mathrm{T}_{\max }$, significant differences were observed for both slopes and intercepts over the whole season and for the fruit growth and post-harvest periods. Similar results were found for the relationships between MDS and $\mathrm{T}_{\text {mid. }}$ The 
relationships between MDS and VPD (either $\mathrm{VPD}_{\max }$ or $\mathrm{VPD}_{\text {mid }}$ ) presented slopes that were not significantly different among years, whereas intercepts showed significantly different values among years (Table 5.2).

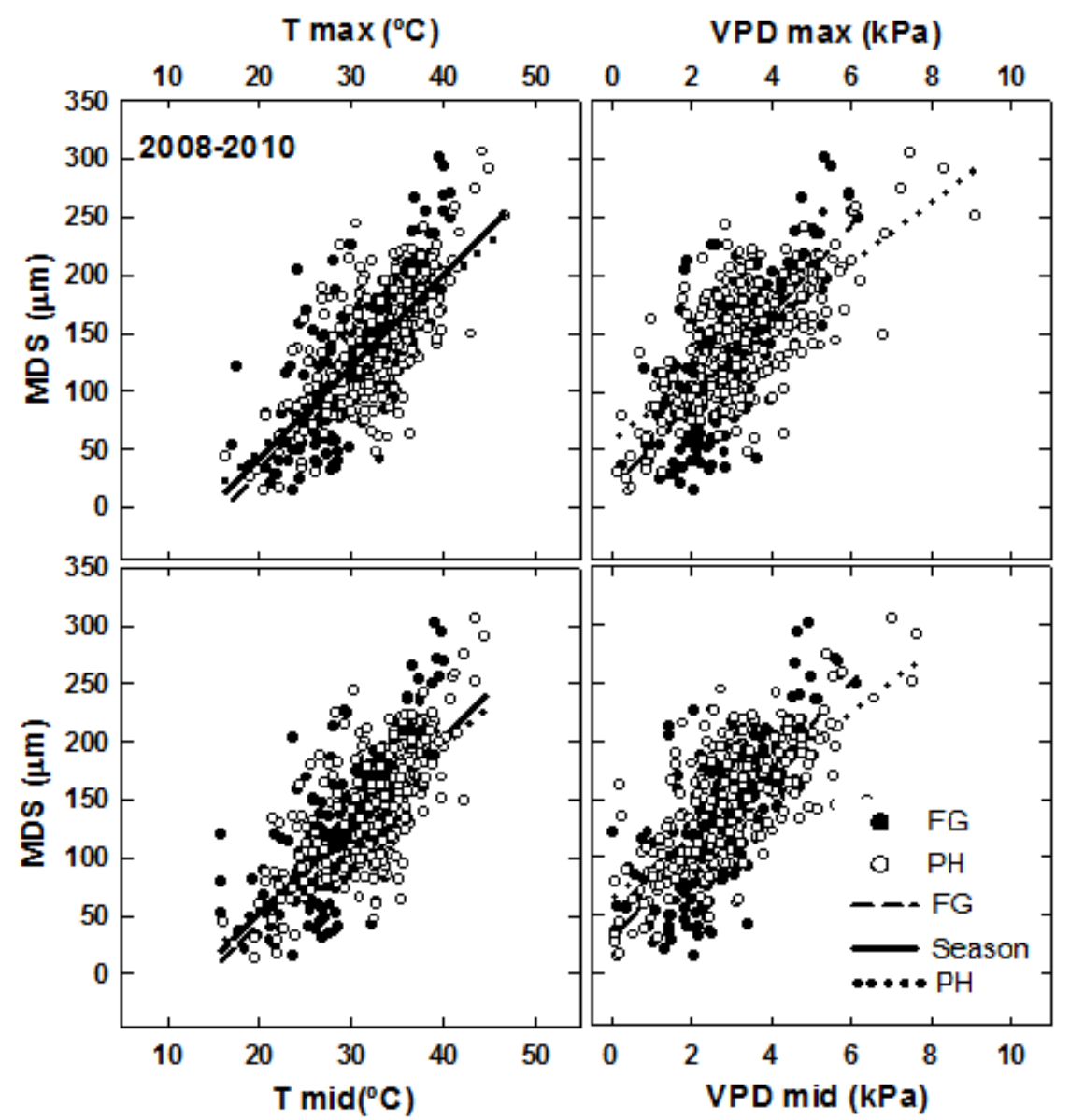

Figure 5.3. Relationships of MDS with the maximum daily temperature $\left(\mathrm{T}_{\max }\right)$, midday temperature $\left(\mathrm{T}_{\operatorname{mid}}\right)$, maximum and midday air vapour pressure deficit $\left(\mathrm{VPD}_{\max }\right.$ and $\mathrm{VPD}_{\text {mid }}$, respectively), pooling data for the three seasons studied (2008-2010). The regression equations for these relationships are presented in Tables 5.2 and 5.3. FG and PH indicate fruit-growth and postharvest stages, respectively.

Under our conditions, the relationships between MDS and the midday and daily maximum temperatures were very similar (Table 5.2 and Figure 5.3). However, maximum temperature is easier to obtain than midday temperature. Moreover, only slight differences in the aspect of the regressions were observed when considering separately the fruit-growth and post-harvest stages (Figure 5.3).

In contrast, VPD showed a greater data dispersion when compared with temperature. This fact caused that the fitted lines between VPD and MDS differed when fruit-growth and post-harvest stages were considered separately (Figure 5.3). 


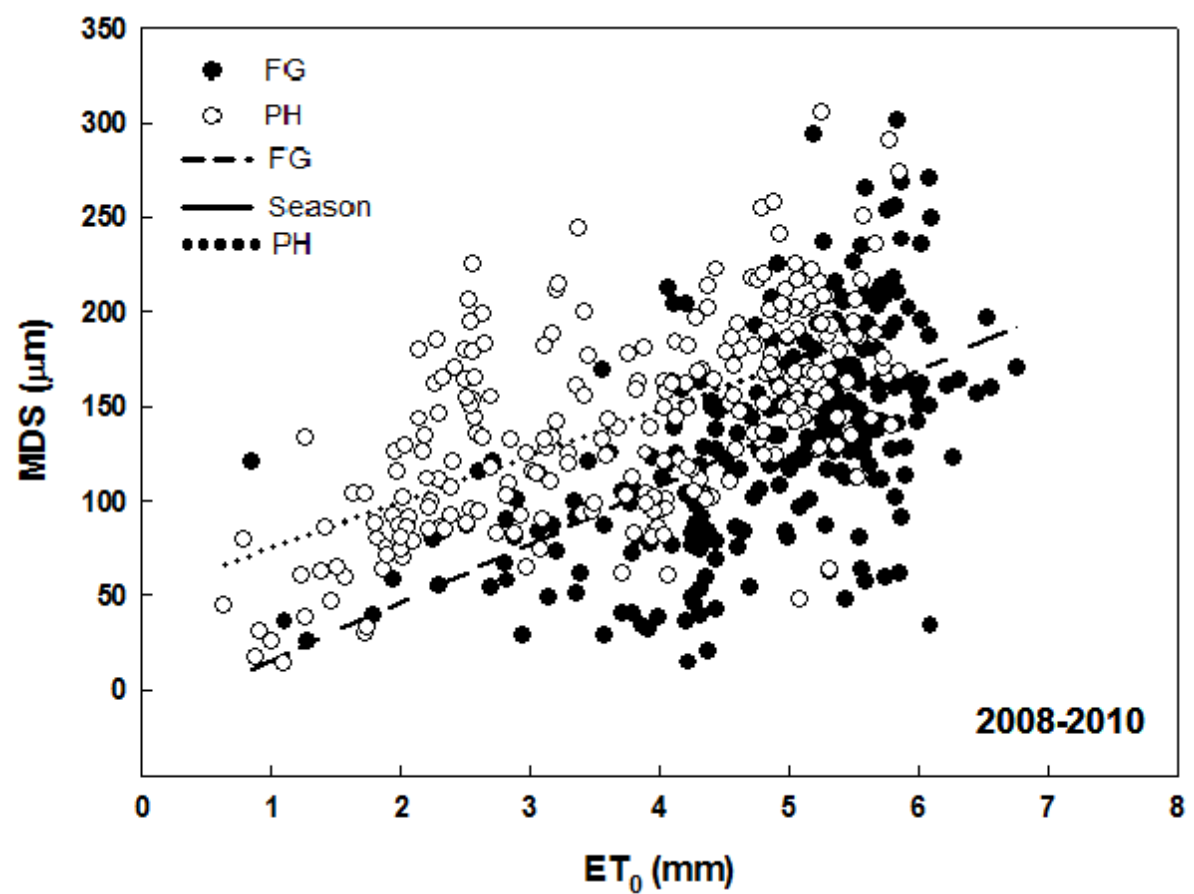

Figure 5.4. Relationship of MDS with potential evapotranspiration $\left(\mathrm{ET}_{0}\right)$ pooling data from the three consecutive seasons studied (2008-2010). The regression equations obtained for each season and the total pool of data are presented in Tables 5.2 and 5.3. FG and PH indicate fruit-growth and postharvest stages, respectively.

Regarding the parameters of the fitted curves for each developmental stage separately (Table 5.3), the highest determination coefficients were observed in 2009 for all the variables, being the maximum one $\left(r^{2}=0.76\right)$ observed for $T_{m i d}$ at fruit-growth. In addition, consistently higher determination coefficients were found for fruit-growth than for post-harvest period (Table 5.3).

When data for all years were pooled together, the rank order of the determination coefficients varied for each developmental stage, being $\mathrm{T}_{\max }=\mathrm{T}_{\operatorname{mid}}>$ $\mathrm{VPD}_{\max }>\mathrm{VPD}_{\min }>\mathrm{ET}_{0}$ for fruit-growth, and $\mathrm{VPD}_{\text {mid }}>\mathrm{VPD}_{\max }>\mathrm{T}_{\operatorname{mid}}>\mathrm{T}_{\max }>\mathrm{ET}_{0}$ for post-harvest (Table 5.3). 
Table 5.2. Parameters of the reference equations for maximum daily trunk shrinkage (MDS) as a function of selected environmental variables during the three seasons studied (2008-2010). ANCOVA results for the comparison of the slopes and intercepts of the fitted curves among the

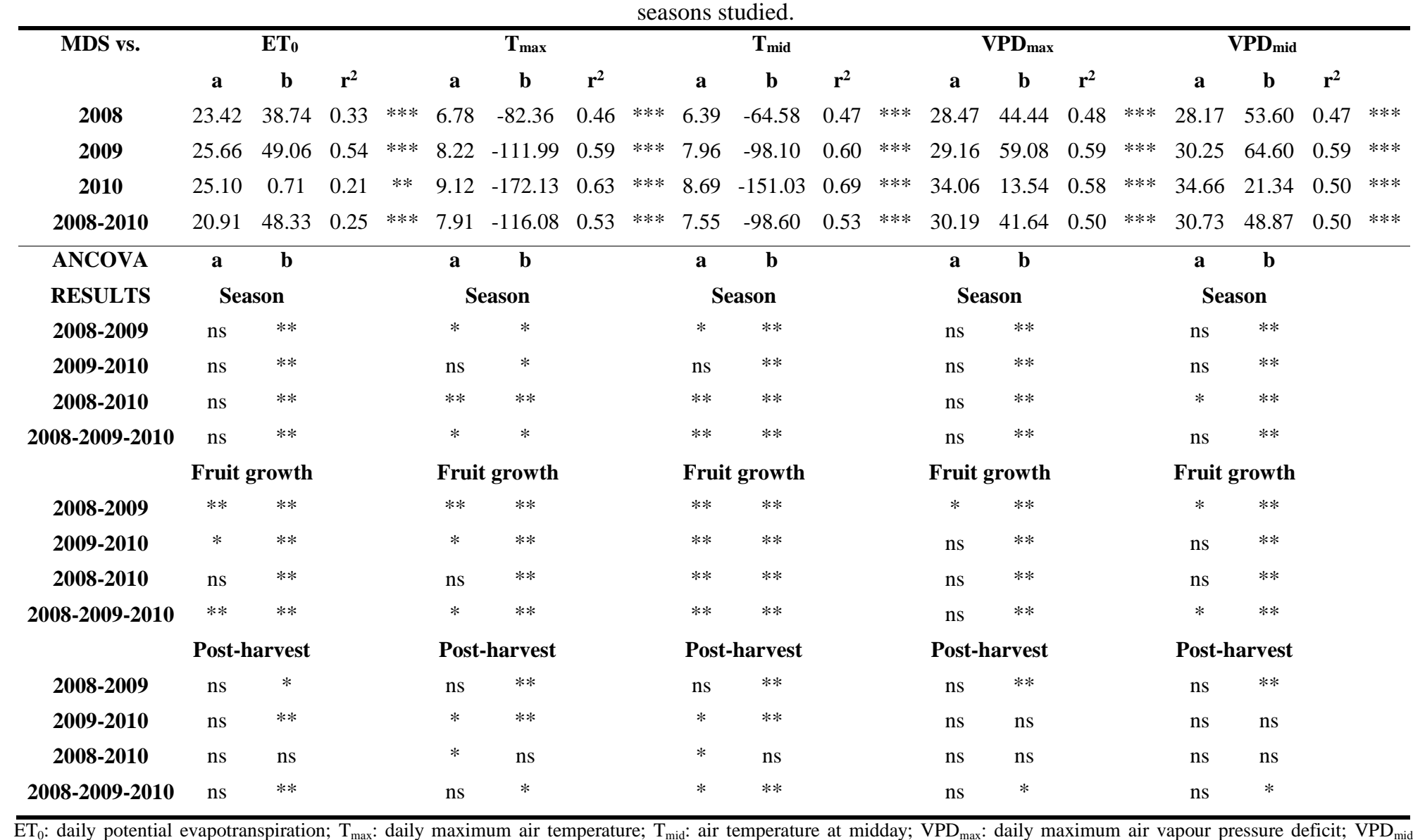

midday air vapour pressure deficit. a: slope of the fitted curve; $\mathrm{b}$ : intercept of the fitted curve; $\mathrm{r}^{2}$ : determination coefficient. $* * *$ Significant at $p<0.01 ; * *$ Significant at $p<0.05$. 
Table 5.3. Parameters of the reference equations for maximum daily trunk shrinkage (MDS) as a function of selected environmental variables during the fruit-growth and post-harvest stages of the three seasons studied (2008-2010).

\begin{tabular}{|c|c|c|c|c|c|c|}
\hline \multirow[b]{2}{*}{$\mathbf{E T}_{\mathbf{0}}$} & \multicolumn{3}{|c|}{ Fruit growth } & \multicolumn{3}{|c|}{ Post-harvest } \\
\hline & $\mathbf{a}$ & b & $\mathbf{r}^{2}$ & $\mathbf{a}$ & b & $\mathbf{r}^{2}$ \\
\hline 2008 & 28.19 & 4.04 & $0.41 * * *$ & 25.37 & 43.32 & $\mathbf{0 . 3 8} * * *$ \\
\hline 2009 & 51.14 & -85.96 & $0.54 * * *$ & 25.04 & 58.11 & $0.43^{* * * *}$ \\
\hline 2010 & 35.07 & -72.08 & $0.46 * * *$ & 32.11 & 3.53 & $0.34 * * *$ \\
\hline 2008-2010 & 30.71 & -15.09 & $0.32 * * *$ & 24.31 & 51.03 & $0.37 * * *$ \\
\hline \multicolumn{7}{|l|}{$\mathbf{T}_{\max }$} \\
\hline 2008 & 7.46 & -96.56 & $0.51 * * *$ & 6.79 & -87.40 & $0.42 * * *$ \\
\hline 2009 & 10.43 & -173.02 & $\mathbf{0 . 7 5} * * *$ & 7.17 & -85.74 & $0.54 * * *$ \\
\hline 2010 & 8.39 & -151.62 & $0.66 * * *$ & 10.65 & -222.32 & $0.45^{* * *}$ \\
\hline 2008-2010 & 9.02 & -147.83 & $0.59 * * *$ & 7.14 & -93.09 & $0.46^{* * * *}$ \\
\hline \multicolumn{7}{|l|}{$\mathbf{T}_{\text {mid }}$} \\
\hline 2008 & 6.88 & -72.19 & $0.52 * * *$ & 6.58 & -76.21 & $0.44 * * *$ \\
\hline 2009 & 10.33 & -162.52 & $0.76 * * *$ & 6.92 & -72.49 & $0.56 * * *$ \\
\hline 2010 & 7.84 & -127.85 & $0.64 * * *$ & 10.79 & -219.45 & $0.50 * * *$ \\
\hline 2008-2010 & 8.48 & -123.47 & $0.59 * * *$ & 6.49 & -81.78 & $0.48 * * *$ \\
\hline \multicolumn{7}{|l|}{ VPD $_{\max }$} \\
\hline 2008 & 31.59 & 38.74 & $0.45 * * *$ & 27.46 & 45.35 & $0.50 * * *$ \\
\hline 2009 & 39.88 & 29.55 & $0.71 * * *$ & 24.86 & 67.60 & $0.56 * * *$ \\
\hline 2010 & 37.77 & -7.36 & $0.58 * * *$ & 23.65 & 65.70 & $0.32 * * *$ \\
\hline 2008-2010 & 38.09 & 15.31 & $0.55 * * *$ & 25.6 & 58.63 & $0.49 * * *$ \\
\hline \multicolumn{7}{|l|}{ VPD $_{\text {mid }}$} \\
\hline 2008 & 28.34 & 55.43 & $0.42 * * *$ & 28.51 & 50.79 & $0.51 * * *$ \\
\hline 2009 & 37.07 & 40.84 & $0.70 * * *$ & 25.81 & 73.17 & $0.55 * * *$ \\
\hline 2010 & 37.86 & 2.90 & $0.56 * * *$ & 25.46 & 66.60 & $0.38 * * *$ \\
\hline 2008-2010 & 36.92 & 28.95 & $\mathbf{0 . 5 3} * * *$ & 26.72 & 63.30 & $0.50 * * * *$ \\
\hline
\end{tabular}

It is noticeable the low determination coefficient value of the relationship between MDS and $\mathrm{ET}_{0}$, either when considering data for the whole season (Table 5.2) or when considering fruit-growth and post-harvest stages separately (Table 5.3). The values of $\mathrm{ET}_{0}$ presented a great variability, which does not correspond with the fluctuations of MDS (Figure 5.4). 
Table 5.4. Parameters of the reference equations for maximum daily trunk shrinkage (MDS) as a function of midday stem water potential $\left(\Psi_{s}\right)$ for the whole season and during the fruit-growth and post-harvest stages of the three years studied (2008-2010). ANCOVA results for the comparison of the slopes and intercepts of the fitted curves among the seasons studied.

\begin{tabular}{|c|c|c|c|c|c|c|c|c|c|c|c|c|}
\hline \multirow[t]{2}{*}{$\operatorname{MDS} v s \Psi_{\mathrm{s}}$} & \multicolumn{3}{|c|}{ Season } & & \multicolumn{3}{|c|}{ Fruit growth } & \multicolumn{5}{|c|}{ Post-harvest } \\
\hline & $\mathbf{a}$ & $\mathbf{b}$ & $\mathbf{r}^{2}$ & & $\mathbf{a}$ & b & $\mathbf{r}^{2}$ & & $\mathbf{a}$ & b & $\mathbf{r}^{2}$ & \\
\hline 2008 & -71.97 & 87.18 & 0.22 & ns & -220.05 & 10.22 & 0.68 & $* * *$ & -18.14 & 135.25 & 0.01 & ns \\
\hline 2009 & -130.8 & 45.55 & 0.18 & ns & -242.65 & -26.13 & 0.62 & $* * *$ & -76.17 & 83.46 & 0.04 & ns \\
\hline 2010 & -231.8 & 62.80 & 0.58 & $* * *$ & -266.37 & -85.08 & 0.65 & $* * *$ & -177.90 & -17.08 & 0.24 & ns \\
\hline 2008-2010 & -122.2 & 44.23 & 0.26 & $* * *$ & -203.87 & -4.98 & 0.51 & $* * *$ & -58.59 & 96.91 & 0.04 & ns \\
\hline
\end{tabular}

\section{ANCOVA RESULTS}

\begin{tabular}{|c|c|c|c|c|c|c|}
\hline \multirow[t]{2}{*}{$\operatorname{MDS} v s \Psi_{\mathrm{s}}$} & \multicolumn{2}{|c|}{ Season } & \multicolumn{2}{|c|}{ Fruit growth } & \multicolumn{2}{|c|}{ Post-harvest } \\
\hline & $\mathbf{a}$ & $\mathbf{b}$ & $\mathbf{a}$ & $\mathbf{b}$ & $\mathbf{a}$ & b \\
\hline 2008-2009 & ns & ns & ns & $* *$ & ns & ns \\
\hline 2009-2010 & $*$ & $*$ & ns & $*$ & ns & ns \\
\hline 2008-2010 & $* *$ & $* *$ & ns & $* *$ & ns & ns \\
\hline 2008-2009-2010 & $* *$ & $* *$ & ns & $* *$ & ns & 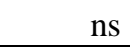 \\
\hline
\end{tabular}

When pooling together data from the three seasons studied, a clear relationship between $\Psi_{\mathrm{s}}$ and MDS was observed during fruit growth (Figure 5.5), higher MDS values corresponding to more negative values of $\Psi_{s}$. However, this relationship did not hold for the post-harvest period and the slope of the fitted line decreased. In fact, these relationships were not significant for the entire seasons of 2008 and 2009; in contrast, during the fruit-growth stage, the correlation between MDS and $\Psi_{s}$ was highly significant with $\mathrm{r}^{2}$ up to 0.68 in 2008, the first year of the experiment (Table 5.4).

At post-harvest, the relationship between both variables was not significant for all the studied years. ANCOVA results proved that slopes and intercepts of the fitted relationships differed from year to year (Table 5.4) when all the data were pooled together, except for the seasons 2008 and 2009. However, the relationship between MDS and $\Psi_{s}$ did not differ for the post-harvest stage and the slopes of the curves were not significantly different among years at the fruit growth stage (Table 5.4). 


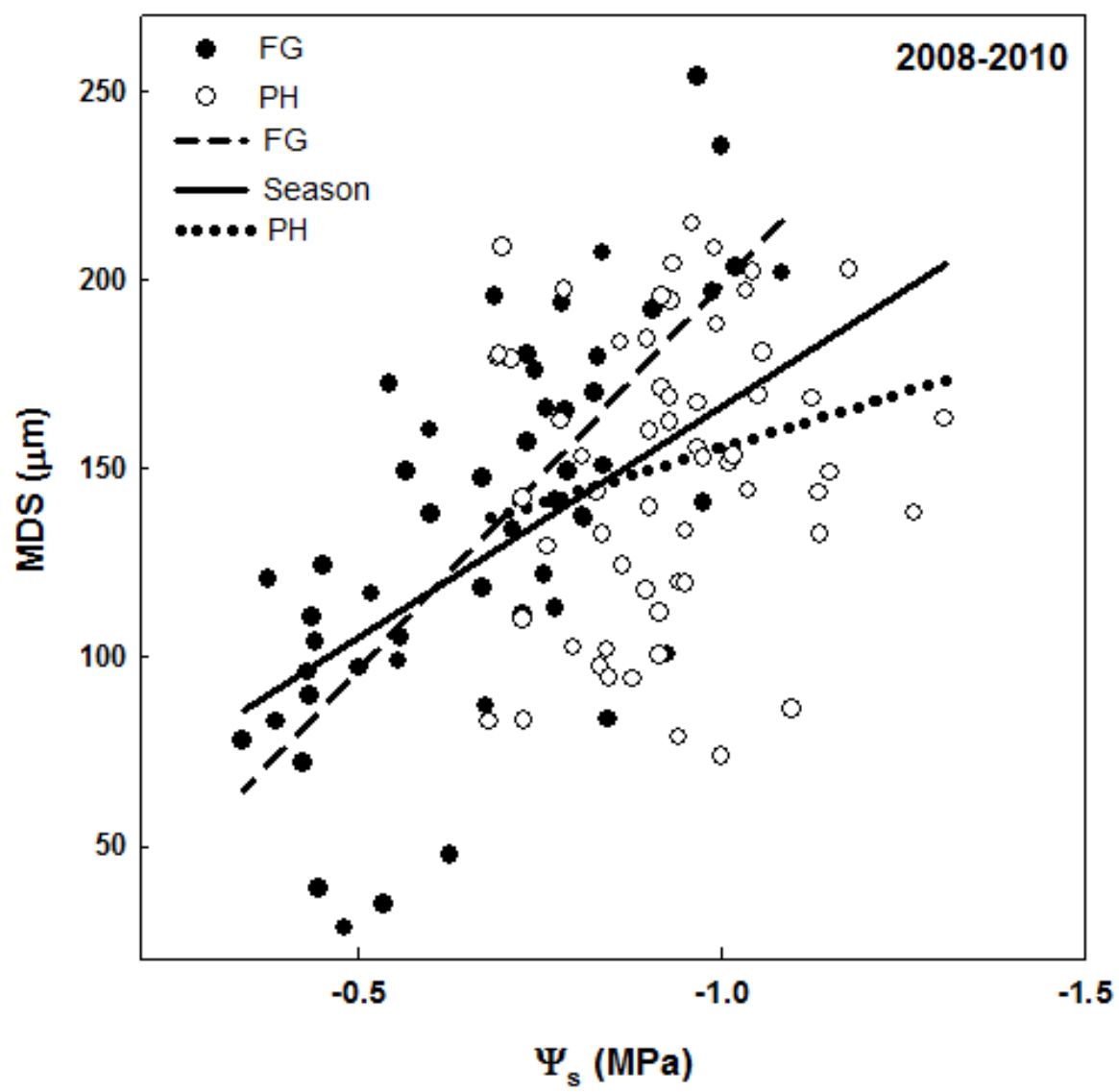

Figure 5.5. Relationship of MDS and midday stem water potential $\left(\Psi_{\mathrm{s}}\right)$ pooling data from the three consecutive seasons studied (2008-2010). The regression equations obtained for each season and the total pool of data are presented in Table 5.4. FG and PH indicate fruit-growth and post-harvest stages, respectively.

\subsection{Discussion}

In the current experiment, trees were well watered and thus grew under nonlimiting soil water conditions (at field capacity), which was proven by increases in trunk diameter and TCSA from year to year (Table 5.1), as well as tree water status $\left(\Psi_{s}\right.$ values). This fact allowed us to obtain baseline relationships between MDS records and meteorological variables closely related to plant water stress and easy to measure, as pointed out by Fernández and Cuevas (2010).

From the five meteorological variables employed in this study, temperature (measured either at midday or the daily maximum) showed the best fit to MDS records for the three growing seasons considered. However, the relationships between both variables differed from year to year (Table 5.2). This result is in agreement with previous reports by Conejero et al. (2007) for an early-maturing peach cultivar, Moriana et al. (2011) for olive, Fereres and Goldhamer (2003) for almond and Ortuño et al. 
(2009) for lemon trees, who found that temperature was the best predictor of MDS for the whole growing season. However, other studies (Intrigliolo and Castel, 2007; Egea et al., 2009) pointed out better adjustments for other climate variables such as VPD.

In addition, when the season was splitted into fruit growth and post-harvest stages, we observed that the parameters (slope and intercept) of the equations relating MDS to temperature varied, being best fitted during the fruit growth stage. This is in accordance with the observations made by Intrigliolo and Castel (2007) in fullyirrigated plum trees and implies that a reference equation obtained under certain conditions should not be applied in subsequent years if tree conditions change. In contrast, Ortuño et al. (2009) concluded for citrus that reference equations between MDS and temperature can be obtained by pooling data across several seasons.

Even though MDS is considered a good indicator of the transpiration stream (Herzog et al., 1995), the relationship of this measurement with VPD was slightly lower than the results obtained with temperature (Tables 5.2 and 5.3). This has been observed by several authors for different fruit trees such as almond (Fereres and Goldhamer, 2003), olive (Corell et al., 2013) and lemon (Ortuño et al., 2009). The midday measurements of temperature presented a better fit than the maximum value, likely due to the fact that midday temperatures are more related to the higher rate of shrinkage occurred around this time of the day.

The presence and amount of fruits may affect MDS records. In our study, the best fits between MDS and climate variables were observed in 2009, the season with the highest fruit load out of the three seasons considered, due to grower's decision associated to market criteria. Moreover, correlations between MDS and the meteorological variables decreased after harvest (Table 5.3), except in 2008 for VPD measurements, as did the slope of the relation between MDS and $\Psi_{s}$ (Figure 5.5 and Table 5.4). Similar results were obtained by Intrigliolo and Castel (2004) for plum and Moriana et al. (2003) for olive. These results indicate some of the difficulties in obtaining reference values useful for irrigation scheduling based exclusively on plant water status parameters.

Furthermore, year-to-year effects of fruit growth on these reference equations were significant (Table 5.3), and this made intercepts and slopes of the equations to be 
rather different among years. However, post-harvest equations were more similar across years. These results suggest that crop load is a very important factor to take into account when using MDS records for irrigation scheduling in this mid-late maturing peach cultivar. This response has already been found in olive and plum trees (Moriana and Fereres, 2004; Intrigliolo and Castel, 2007) but not in citrus (Ortuño et al., 2009).

As pointed out by Ortuño et al. (2010), this behaviour might be related with the idea that stem diameter variations should be considered not only in the context of the water balance of the plant but also in the tree carbon balance (Sevanto et al., 2003; Daudet et al., 2005), because in some woody crop species the sugar content of woody tissues changes as a function of the presence or absence of sinks, including fruits (Flore and Layne, 1997). For this reason, it is of paramount importance to consider crop load when using MDS as a water stress indicator. Consequently, specific reference equations must be used to adjust for tree crop load when using MDS to determine plant water status and irrigation requirements (Intrigliolo and Castel, 2007).

Surprisingly, daily $\mathrm{ET}_{0}$ was the climate variable showing the poorest correlation with MDS records (Tables 5.2 and 5.3, Figure 5.4). This has been previously observed by a number of authors who found better correlations between MDS and climate variables other than $\mathrm{ET}_{0}$, such as VPD (Goldhamer and Fereres, 2004; Egea et al., 2009) or midday temperature (Ortuño et al., 2009; Moriana et al., 2011), coinciding with our results and suggesting that for woody species $\mathrm{ET}_{0}$ would not be the best criterium for determining crop water requirements using reference equations. However, $\mathrm{ET}_{0}$ is the most widely used criterium for assessing crop water requirements, calculated through the Penman-Monteith equation (Allen et al., 1998), and some authors found it significantly correlated with MDS records (Ortuño et al., 2006; Fereres and Goldhamer, 2003).

Finally, it is important to point out that data obtained during the course of the entire experiment and shown in Table 5.4 and Figure 5.5 suggest that there was not a single unique relationship between MDS and $\Psi_{s}$ that was valid for the whole period. This behaviour is similar to that previously observed in peach (Marsal et al., 2002), plum (Intrigliolo and Castel, 2006) or pomegranate (Intrigliolo et al., 2011). In these experiments and the one reported in the current work, the relationship between MDS and $\Psi_{s}$ changed in concordance with changes in the fruit growth pattern or fruit 
removal. In the case reported here, better fits were obtained for data recorded during fruit growth than at post-harvest. Intrigliolo et al. (2011) explained this behaviour because of changes in tissue elasticity since it is generally accepted that tissue age affects its elasticity, older tissues being less elastic (higher resistance to shrinkage; Tyree and Jarvis, 1982). Therefore, the lower MDS for a given $\Psi_{s}$ value obtained late in the season may be due to less elastic, older tissues.

In summary, results presented here indicate that MDS records of a mid-maturing peach cultivar are better related to midday and maximum temperature than to other meteorological variables during fruit growth; and to VPD during the postharvest period. However, these relationships were greatly affected by the conditions of each year, tree phenological stage and crop load. Therefore, the growers must be aware of these factors when using MDS values for irrigation scheduling.

\section{Acknowledgements}

This study was supported by IRRIQUAL (EU-FP6-FOOD-CT-2006-023120) and SIRRIMED (KBBE-2009-1-2-03, PROPOSAL N॰245159) projects. We are also grateful to two SENECA projects (05665/PI/07 and 11872/PI/09) and CONSOLIDER INGENIO 2010 (MEC CSD2006-0067) and CICYT (AGL2010-17553) projects for providing funds to finance this research.

\subsection{References}

Allen, R.G., Pereira, L.S., Raes, D., Smith, M., 1998. Crop evapotranspiration. Guidelines for computing crop water requirements. FAO Irrigation and Drainage paper No 56, Rome, Italy. 300 pp.

Conejero, W., Alarcón, J.J., García-Orellana, Y., Abrisqueta, J.M., Torrecillas, A., 2007. Daily sap flow and maximum daily trunk shrinkage measurements for diagnosing water stress in early maturing peach trees during the post-harvest period. Tree Physiol. 27, 1753-1759.

Conejero, W., Mellisho, C.D., Ortuño, M.F., Moriana, A., Moreno, F., Torrecillas, A., 2011. Using trunk diameter sensors for regulated deficit irrigation scheduling in early maturing peach trees. Environ. Exp. Bot. 71, 409-415.

Corell, M., Girón, I.F., Moriana, A., Dell'Amico, J., Morales, D., Moreno, F., 2013. Extrapolating base-line trunk shrinkage reference equations across olive orchards. Agric. Water Manage. 126, 1-8. 
Daudet, F.A., Améglio, T., Cochard, H., Archilla, O., Lacointe, A., 2005. Experimental analysis of the role of water and carbon in tree stem diameter variations. J. Exp. Bot. 56, 135-144.

De la Rosa, J.M., Conesa, M.R., Domingo, R., Torres, R., Pérez-Pastor, A., 2013. Feasibility of using trunk diameter fluctuation and stem water potential reference lines for irrigation scheduling of early nectarine trees. Agric. Water Manage. $126,133-141$.

Egea, G., Pagán, E., Baille, A., Domingo, R., Nortes, P.A., Pérez-Pastor, A., 2009. Usefulness of establishing trunk diameter based reference lines for irrigation scheduling in almond trees. Irrig. Sci. 27, 431-441.

Fereres, E., Goldhamer, D.A., 2003. Suitability of stem diameter variations and water potential as indicators for irrigation scheduling of almond trees. J. Hort. Sci. Biotechnol. 78, 139-144.

Fereres, E., Soriano, M.A., 2007. Deficit irrigation for reducing agricultural water use. J. Exp. Bot. 58, 147-159.

Fernández, J.E., Cuevas, M.V., 2010. Irrigation scheduling from stem diameter variations: A review. Agric. Forest Meteorol. 150, 135-151.

Flore, J.A., Layne, D.R., 1997. Prunus. In: Zamski, E., Scheffer, H. (Eds.), Photoassimilates Distribution in Plants and Crops: Sink-Source Relationship. Marcel Dekker, New York, pp. 825-849.

Goldhamer, D.A., Fereres, E., 2001. Irrigation scheduling protocols using continously recorded trunk diameter measurements. Irrig. Sci. 20, 115-125.

Goldhamer, D.A., Fereres, E., Salinas, M., 2003. Can almond trees directly dictate their irrigations needs? Calif. Agric. 57, 138-144.

Goldhamer, D.A., Fereres, E., 2004. Irrigation scheduling of almond trees with trunk diameter sensors. Irrig. Sci. 23, 11-19.

Herzog, K.M., Häsler, R., Thum, R., 1995. Diurnal changes in the radius of a subalpine Norway spruce stem: their relation to the sap flow and their use to estimate transpiration. Trees 10, 94-101.

Intrigliolo, D.S., Castel, J.R., 2004. Continuous measurement of plant and soil water status for irrigation scheduling in plum. Irrig. Sci. 23, 93-102.

Intrigliolo, D.S., Castel, J.R., 2006. Usefulness of diurnal trunk shrinkage as a water stress indicator in plum trees. Tree Physiol. 26, 303-311.

Intrigliolo, D.S., Castel, J.R., 2007. Crop load affects maximum daily trunk shrinkage of plum trees. Tree Physiol. 27, 89-96.

Intrigliolo, D.S., Puerto, H., Bonet, L., Alarcón, J.J., Nicolás, E., Bartual, J., 2011. Usefulness of trunk diameter variations as continuous water stress indicators of pomegranate (Prunus granatum) trees. Agric. Water Manage. 98, 1462-1468. 
Katerji, N., Tardieu, F., Bethenod, O., Quetin, P., 1994. Behaviour of maize stem diameter during drying cycles: comparison of two methods for detecting water stress. Crop Sci. 34, 165-169.

MAGRAMA, 2015. Anuario de estadística Avance 2014. Ministerio de Agricultura, Alimentación y Medio Ambiente.

Marsal, J., Gelly, M., Mata, M., Arbones, J., Rufat, J., Girona, J., 2002. Phenology and drought affects the relationship between daily trunk shrinkage and midday stem water potential of peach trees. J. Hortic. Sci. Biotechnol. 77, 411-417.

Moreno, F., Conejero, W., Martín-Palomo, M.J., Girón, I.F., Torrecillas, A., 2006. Maximum daily trunk shrinkage reference values for irrigation scheduling in olive trees. Agric. Water Manage. 84, 290-294.

Moriana, A., Orgaz, F., Fereres, E., Pastor, M., 2003. Yield responses of a mature olive orchard to water deficits. J. Am. Soc. Hortic. Sci. 128, 425-431.

Moriana, A., Fereres, E., 2004. Establishing reference values of trunk diameter fluctuations and stem water potential for irrigation scheduling of olive trees. Acta Hortic. 664, 407-412.

Moriana, A., Moreno, F., Girón, I.F., Conejero, W., Ortuño, M.F., Morales, D., Corell, M., Torrecillas, A., 2011. Seasonal changes of maximum daily shrinkage reference equations for irrigation scheduling in olive trees: Influence of fruit load. Agric. Water Manage. 99, 121-127.

Naor, A., 2006. Irrigation scheduling and evaluation of tree water status in deciduous orchards. Horticultural Reviews 32, 111-165.

Nicolás, E., Torrecillas, A., Ortuño, M.F., Domingo, R., Alarcón, J.J., 2005. Evaluation of transpiration in adult apricot trees from sap flow measurements. Agric. Water Manage. 72, 131-145.

Ortuño, M.F., García-Orellana, Y., Conejero, W., Ruiz-Sánchez, M.C., Mounzer, O., Alarcón, J.J., Torrecillas, A., 2006. Relationships between climatic variables and sap flow, stem water potential and maximum daily trunk shrinkage in lemon trees. Plant Soil 279, 229-242.

Ortuño, M.F., Brito, J.J., García-Orellana, Y., Conejero, W., Torrecillas, A., 2009. Maximum daily trunk shrinkage and stem water potential baselines for irrigation scheduling of lemon trees. Irrig. Sci. 27, 121-127.

Ortuño, M.F., Conejero, W., Moreno, F., Moriana, A., Intrigliolo, D.S., Biel, C., Mellizo, C.D., Pérez-Pastor, A., Domingo, R., Ruiz-Sánchez, M.C., Casadesus, J., Bonany, J., Torrecillas, A., 2010. Could trunk diameter sensors be used in woody crops for irrigation scheduling? A review of current knowledge and future perspectives. Agric. Water Manage. 97, 1-11.

Pagán, E., Pérez-Pastor, A., Domingo, R., Conesa, M.R., Caro, M., 2012. Suitability of trunk diameter reference lines for irrigation scheduling with saline water in late mandarin trees with different crop load. Agric. Water Manage. 111, 11-19. 
Remorini, D., Massai, R., 2003. Comparison of water status indicators for young peach trees. Irrig. Sci. 22, 39-46.

Sevanto, S., Vesala, T., Perämäki, M., Nikinmaa, E., 2003. Sugar transport together with environmental conditions controls time lags between xylem and stem diameter changes. Plant Cell Environ. 26, 1257-1265.

Shackel, K.A., Ahmadi, H., Biasi, W., Buchner, R., Goldhamer, D., Gurusinghe, S., Hasey, J., Kester, D., Krueger, B., Lampinen, B., McGourty, G., Micke, W., Mitcham, E., Olson, B., Pelletrau, K., Philips, H., Ramos, D., Schwankl, L., Sibbett, S., Snyder, R., Southwick, S., Stevenson, M., Thorpe, M., Weinbaum, S., Yeager, J., 1997. Plant water status as an index of irrigation need in deciduous fruit trees. HortTechnology 7 (1), 23-29.

Turner, N.C., 1981. Techniques and experimental approaches for the measurement of plant water status. Plant Soil 58, 339-366.

Tyree, M.T., Jarvis, P.G., 1982. Water relations and carbon assimilation. In: Lange, O.L., Nobel, P.S., Osmond, C.B., Ziegler, H. (Eds.), Physiological Plant Ecology II. Springer Verlag, Berlin. Pp. 35-78.

Westwood M.N., 1988. Temperate-Zone Pomology, Ed. Timber Press, Portland, CO, USA. 


\section{USEFULNESS OF MAXIMUM DAILY TRUNK SHRINKAGE FOR ESTIMATING WATER NEEDS AND REGULATED DEFICIT IRRIGATION SCHEDULING IN PEACH TREES}





\title{
6. USEFULNESS OF MAXIMUM DAILY TRUNK SHRINKAGE FOR ESTIMATING WATER NEEDS AND REGULATED DEFICIT IRRIGATION SCHEDULING IN PEACH TREES
}

\begin{abstract}
Water scarcity and the increase of water use in many socio-economical sectors leads to an urgent need to improve irrigation management. In this sense, the scientific community is immersed in the search to develop new and precise technologies for scheduling irrigation, especially in arid and semi-arid areas, such as the South East of Spain. For this reason, we studied the possibility of scheduling regulated deficit irrigation (RDI) using maximum daily shrinkage (MDS) measurements on peach trees [Prunus persica (L.) Batsch cv. "Catherine"] in Murcia (Spain) during three years. Three irrigation treatments were established: a control treatment $\left(\mathrm{C}_{\mathrm{ETc}}\right)$, irrigated above the estimated crop water requirements, and two treatments based on measurements of maximum daily shrinkage signal intensity (MDS SI). Precision treatment $\left(\mathrm{C}_{\mathrm{MDS}}\right)$, using a threshold value of MDS SI=1 during all the period and a deficitary treatment (RDI) using MDS SI=1 all the period except for stage II and postharvest where MDS SI=2. The low coefficients of variation of MDS SI indicated that the information generated by these measurements under $\mathrm{C}_{\mathrm{MDS}}$ and RDI was sufficient to adjust the irrigation schedule to maintain MDS SI close to the selected threshold values. Hence, peach tree irrigation scheduling can be exclusively based on MDS measurements. Maintaining the MDS SI close to unity throughout the growing season can be a useful tool to estimate $\mathrm{K}_{\mathrm{c}}$ values when these are not available. The RDI treatment significantly reduced pruning weight, but not significant differences were found in terms of reproductive growth and yield. Moreover, RDI increased water productivity, fruit quality, since soluble solids content and maturity index were higher in the RDI treatment compared to $\mathrm{C}_{\mathrm{ETc}}$ and $\mathrm{C}_{\mathrm{MDS}}$.
\end{abstract}

Keywords: Prunus persica, trunk diameter fluctuations, yield, signal intensity, water productivity. 


\subsection{Introduction}

Spain is the second largest peach [Prunus persica (L.) Bastch] producer in the world, and this is the first stone fruit crop in Spain. The area devoted to peach tree varieties with low cold requirements has increased in recent years, especially in southern regions. Peach tree plantations are located in the Mediterranean area, being Murcia (SE Spain) the third main peach production area in the country, yielding 249,500 t per year, 19\% of the Spanish production, approximately (MAGRAMA, 2015). More than $70 \%$ of the surface devoted to peach cultivation in the region is managed under irrigation conditions.

Irrigated agriculture is the primary water user worldwide, reaching a proportion that exceeds $70-80 \%$ of the total in arid and semi-arid regions. This, added to an increasing pressure on water resources due to the competition for water between agriculture, industry and population, creates an urgent need to increase irrigation water productivity and to develop and provide new tools and water conservation methods in agriculture. There is a growing awareness of the need for sustainable irrigation planning in commercial orchards. This implies a continuous reduction in water inputs throughout the growing season that can only be beneficial as long as fruit quality and yield can be maintained (Fereres and Soriano, 2007). For this reason, it is of paramount importance to determine the tree water status throughout the year in order to rapidly detect any water stress and correctly manage irrigation over the growing season.

The use of plant-based water status indicators has become very popular for planning precise irrigation, because plant water status controls many physiological processes and crop productivity, this information can be highly useful in irrigation scheduling (Fernández and Cuevas, 2010; Ortuño et al., 2010).

In this sense, several plant water stress indicators such as the plant leaf and stem water potentials have been tested for schedule irrigation. However, these measurements cannot be easily automated and there is a need to look for other tools that continuously monitor plant water status. In this respect, the continuous measurements made by lineal variable diameter transducers (LVDT) of trunk diameter fluctuations are easily recordable with a datalogger, and could provide real-time knowledge of the plant water stress levels reached in the implementation of regulated deficit irrigation strategies. From these records, two indexes are normally obtained, the maximum diurnal trunk 
shrinkage (MDS) and the trunk growth rate (TGR). Particularly, MDS has been shown to have the potential to serve as plant water stress indicator (Fernández and Cuevas, 2010).

Because plants are in the middle of the soil-plant-atmosphere, plant water status is the result of soil water availability and evaporative demand. Therefore, absolute water stress indicator values recorded without considering the evaporative demand might be meaningless. For this reason, it is better to use the concept of signal intensity (SI) for irrigation scheduling, normalizing an indicator's absolute values with respect to values under non-limiting soil water conditions (Naor and Cohen, 2003; Ortuño et al., 2006). The SI for water stress indicators is a dimensionless variable, where values above unity indicate water stress levels, while SI values of unity indicate the absence of irrigationrelated stress (Goldhamer and Fereres, 2004).

Previous research confirmed that MDS SI is a suitable parameter for irrigation scheduling (Vélez et al., 2007 in orange trees; Ortuño et al., 2009 in lemon trees; Conejero et al., 2011 in peach trees; Puerto et al., 2013 in almond trees and De la Rosa et al., 2014 in nectarines trees).

In the current work, MDS SI was used as a potential indicator for irrigation scheduling in peach trees and the impact of irrigation strategies based on MDS SI on yield and fruit quality were evaluated:

1) To quantify peach tree water requirements maintaining MDS SI values close to unity during crop development, in order to determine crop factors $\left(\mathrm{K}_{\mathrm{c}}\right)$ in this species.

2) To evaluate a methodology for scheduling peach orchard irrigation based on MDS SI thresholds as a water stress indicator for triggering irrigation, and assess the effects of this RDI on peach tree physiology, yield and fruit quality, as well as water savings, when compared to a fully irrigated control.

\subsection{Materials and methods}

\section{Site description and plant material}

The experiment was conducted over three consecutive years (2008-2010) in a 0.5 ha plot of a commercial orchard located in Mula valley, Murcia, SE Spain $\left(37^{\circ} 55^{\prime} \mathrm{N}\right.$, $1^{\circ} 25^{\prime} \mathrm{W}, 360 \mathrm{~m}$ above sea level). The soil at the site is calcareous, stony, with a sandy- 
loam texture, low organic matter content and its available water capacity is $0.25 \mathrm{~m}^{3} \mathrm{~m}^{-3}$, approximately. The climate of the area is typically Mediterranean, with mild winters, hot dry summers and low rainfall amounts.

Plant material consisted of peach trees [Prunus persica (L.) Batsch cv."Catherine"] planted in 1999 and grafted on GF-677 rootstock. Spacings were 4 m x $6 \mathrm{~m}$. Hand thinning was used to space fruitlets $20 \mathrm{~cm}$ along the fruit bearing shoots in order to achieve a commercial crop load. Pest control was that commonly used by the growers and all treatments received the same fertilization $\left(\mathrm{N}-\mathrm{P}_{2} \mathrm{O}_{5}-\mathrm{K}_{2} \mathrm{O}\right)$, applied through the drip irrigation system $\left(275-125-200 \mathrm{~kg} \mathrm{ha}^{-1}\right.$ year $\left.^{-1}\right)$ over the study period.

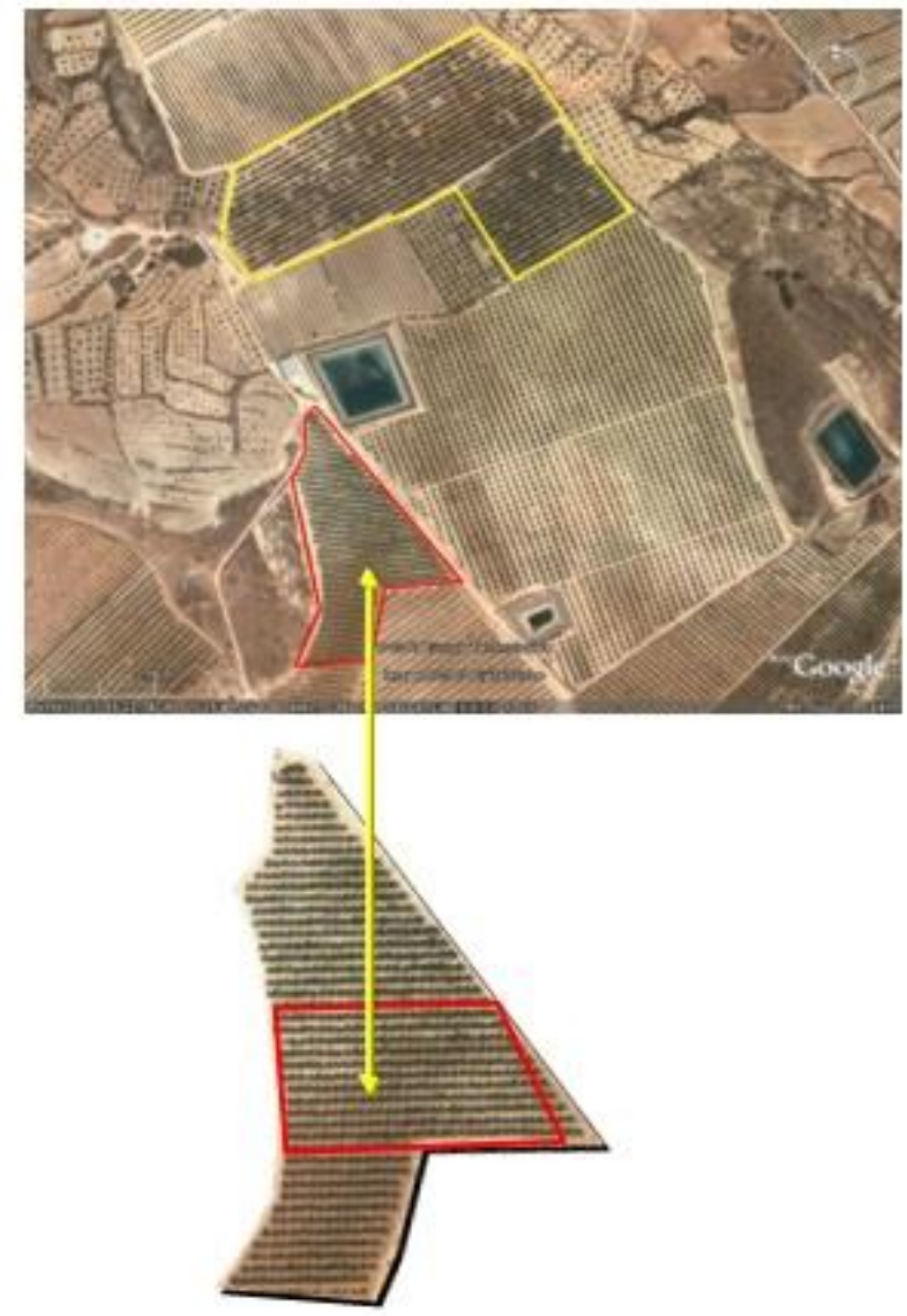

Picture 6.1. Plot location. (Source Google Maps). 


\section{Irrigation treatments and experimental design}

Irrigation water was supplied through a drip irrigation system, one pipeline for each row, with three emitters per tree (delivering $4 \mathrm{~L} \mathrm{~h}^{-1}$ each). The irrigation water was considered of good quality with a very low electrical conductivity $\left(0.6 \mathrm{dS} \mathrm{m}^{-1}\right)$.

Crop irrigation requirements for control treatment were scheduled weekly according to daily $\mathrm{ET}_{0}$, calculated using the Penman-Monteith equation (Allen et al., 1998), and a local crop factor based on the time of the year and used in this region at commercial level. Monthly average values of $\mathrm{K}_{\mathrm{c}}$ employed for the control treatment from April to October were 0.73, 0.83, 0.94, 0.96, 0.39, 0.39 and 0.39, respectively. A total of 192 trees were used in this study. The experiment was laid out in completely randomized blocks with 4 replications (16 trees each). The four central trees were used for measurements, and the other 12 trees acted as guard.

\begin{tabular}{|c|c|c|c|c|c|c|c|c|c|c|}
\hline \multicolumn{3}{|c|}{ WINTER } & \multicolumn{3}{|c|}{ SPRING } & \multicolumn{3}{|c|}{ SUMMER } & \multicolumn{2}{|c|}{ AUTUMN } \\
\hline JANUARY & \begin{tabular}{|l|} 
FEBRUARY \\
\end{tabular} & MARCH & APRIL & MAY & JUNE & JULY & AUGUST & \begin{tabular}{|l|} 
SEPTEM BER \\
\end{tabular} & OCTOBER & \begin{tabular}{|l|l|} 
NOVEMB ER & DECEM BER \\
\end{tabular} \\
\hline
\end{tabular}

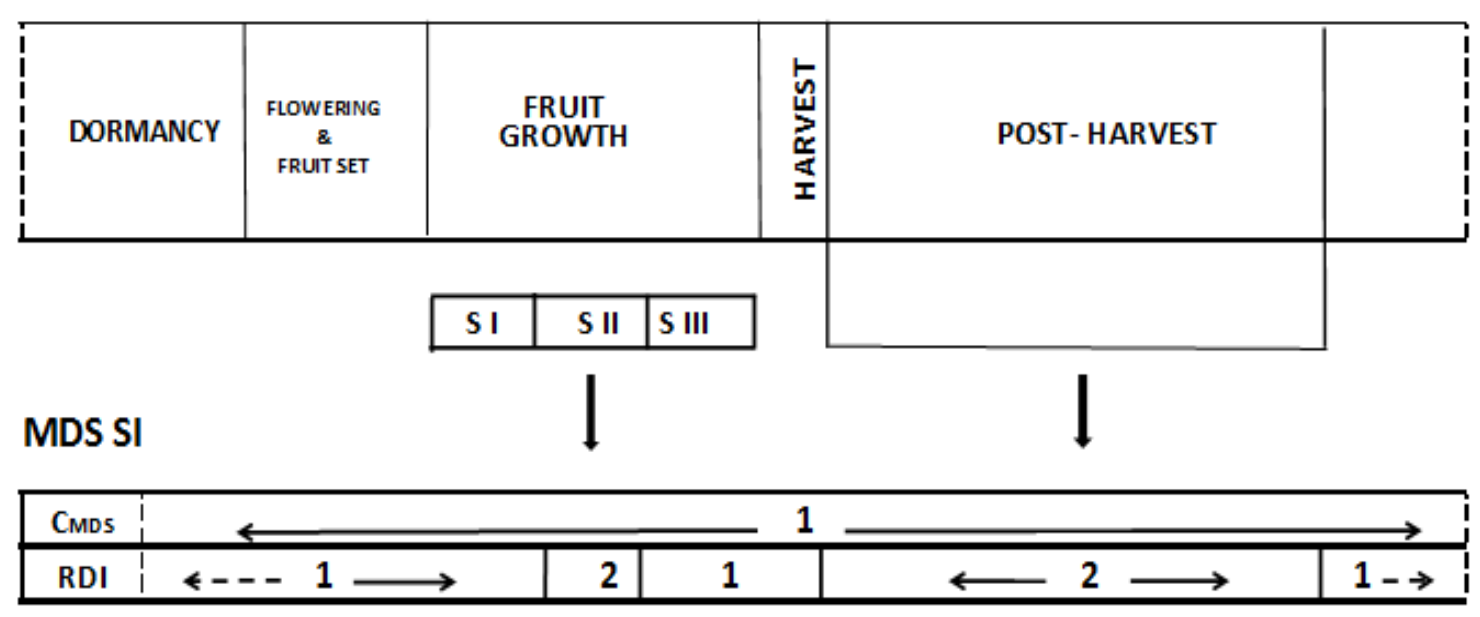

Figure 6.1. Precision ( $\mathrm{C}_{\mathrm{MDS}}$ ) and Regulated Deficit Irrigation (RDI) strategies based on threshold values for maximum daily shrinkage signal intensity (MDS SI) applied in stage II (S II) of fruit growth and postharvest during the three years of the experiment.

Three irrigation treatments were applied based on peach tree phenological stage: Control plants $\left(\mathrm{C}_{\mathrm{ETc}}\right)$ were daily irrigated above the estimated crop evapotranspiration in order to obtain non-limiting soil water conditions (average $120 \% \mathrm{ET}_{\mathrm{c}}$ in three years of experiments), and two RDI strategies that consisted of a full irrigation during critical periods, and water restrictions during the non-critical periods of crop development 
(stage II of fruit growth and post-harvest periods) (Figure 6.1). These RDI treatments were:

a) Precision treatment $\left(\mathrm{C}_{\mathrm{MDS}}\right)$ in which the MDS SI values were maintained close to unity during crop growth.

b) Deficit treatment (RDI) consisting of full irrigation during critical periods (values of MDS SI close the unity), which corresponded to the flowering and fruit set, stages I and III of fruit growth, and dormancy period, and subjected to water shortage during the non-critical periods of crop development corresponded to stage II of fruit growth and post-harvest periods (MDS SI values close to 2 for these two stages).

The irrigation rate was decreased by $10 \%$ when the MDS SI did not exceed the threshold value on at least two out of three consecutive days, and increased by $10 \%$ when the MDS SI exceeded the threshold value on at least two out of three consecutive days.

This irrigation protocol was based on that proposed by Goldhamer and Fereres (2001) for mature trees under high frequency irrigation. Irrigation was controlled automatically by a head unit programmer and the amounts of water applied for each irrigation treatment were measured with in-line flowmeters placed in each experimental plot.

\section{Field and laboratory determinations}

\section{Climate data}

Climate data were recorded at an automatic weather station placed within the experimental orchard. Data on air temperature (maximum, minimum and average), solar radiation, air relative humidity, rainfall and wind speed $2.5 \mathrm{~m}$ above the soil surface, were collected every $15 \mathrm{~min}$. These data were used for calculating $\mathrm{ET}_{0}$ and crop water requirements. The evolution of these parameters over the study period is shown in figure 6.2. 


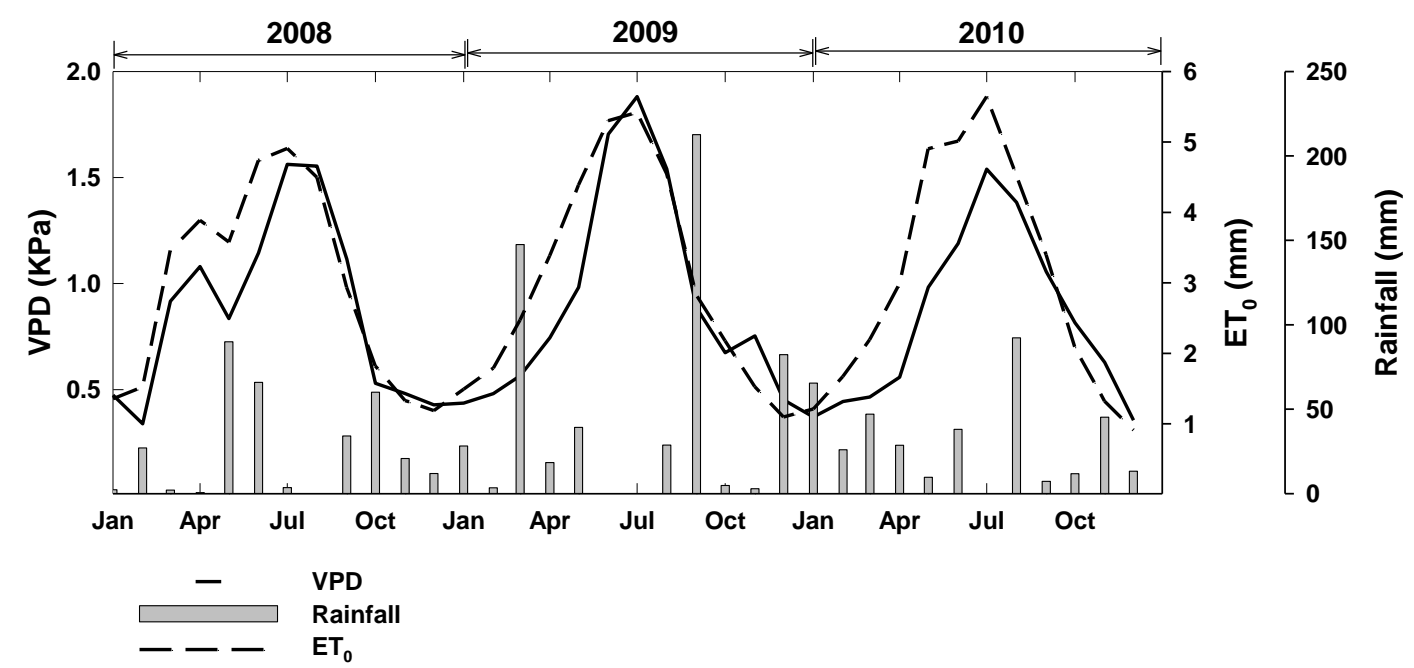

Figure 6.2. Reference evapotranspiration $\left(\mathrm{ET}_{0}, \mathrm{~mm}\right)$, rainfall $(\mathrm{mm})$ and vapour pressure deficit (VPD, KPa) in Fuente Librilla. Monthly values from data collected during 2008, 2009 and 2010.

\section{Soil water content}

The volumetric soil water content $\left(\theta_{\mathrm{v}}, \mathrm{m}^{3} \mathrm{~m}^{-3}\right)$ of the top $0.2 \mathrm{~m}$ of the soil profile was measured by time domain reflectometry (TDR) using a Tektronix device (Model 1502C, Tektronix Inc., OR), as described by Moreno et al. (1996). The $\theta_{\mathrm{v}}$ from $0.2 \mathrm{~m}$ down to a maximum depth of $1.0 \mathrm{~m}$ was measured every $0.1 \mathrm{~m}$ using a neutron probe (Model 4300, Troxler Electronic Laboratories Inc., NC), in access tubes installed $1.0 \mathrm{~m}$ from the trees and beside the emitters. The probes were placed at $0.2 \mathrm{~m}$ from the perperdicular emitter and next to the TDR rods. Measurements using one neutron probe and TDR per experimental plot (3 replications per treatment) were taken in the morning, every 7 to 15 days, during the experimental period.

\section{Stem water potential}

Midday stem water potential $\left(\Psi_{\mathrm{s}}\right)$ was measured using a pressure chamber (Soil Moisture Equip. Corp, model 3000, Santa Barbara, CA, USA) following the procedures described by Turner (1981). Measurements were performed on mature leaves (one per tree) from the north face of the trunk, in the four central trees of each experimental plot. Leaves were enclosed in plastic bags covered with aluminum foil at least two hours prior to the measurements, which were carried out between 12:00 and 13:00 h solar time. 


\section{Trunk diameter fluctuations}

The micrometric trunk diameter fluctuations (TDF) were measured throughout the experimental period in four trees per treatment (one tree from each experimental plot), using a set of linear variable displacement transducers (LVDT) (Solartron Metrology, Bognor Regis, UK, model DF $\pm 2.5 \mathrm{~mm}$, accuracy $\pm 10 \mu \mathrm{m}$,) attached to the trunk, with a special bracket made of Invar, an alloy of $\mathrm{Ni}$ and $\mathrm{Fe}$ with a thermal expansion coefficient close to zero (Katerji et al., 1994), and aluminium. Sensors were placed on the north side and were covered with silver thermoprotected foil to prevent heating and wetting of the device. In order to eliminate measurement noise, old bark was carefully eliminated with a sharpened knife. Then, TDF measurements included only the contributions of the phloem and xylem. Measurements were taken every $30 \mathrm{~s}$ and the 2 dataloggers (model CR1000, Campbell Scientific, Inc., Logan, USA) were programmed to report 15 min means. Maximum daily trunk shrinkage (MDS) was calculated daily as the difference between maximum and minimum daily trunk diameter (Goldhamer and Fereres, 2001).

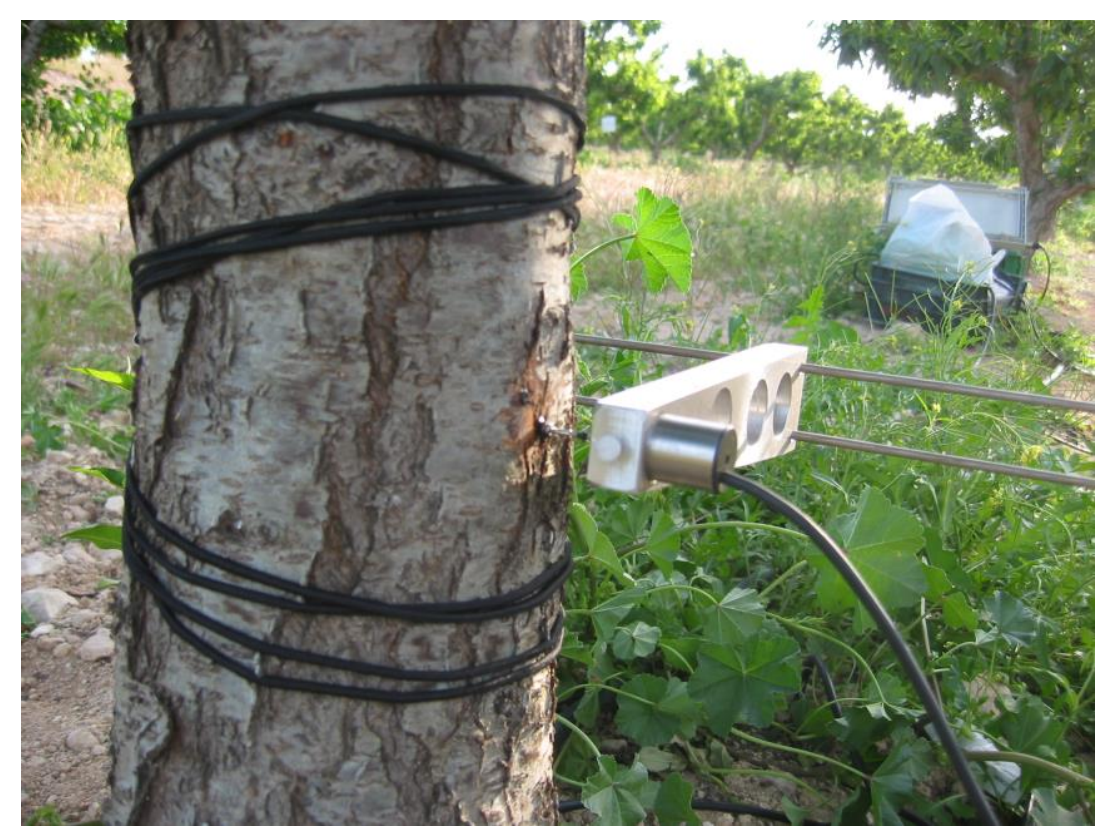

Picture 6.2. LVDT sensor installed in peach "Catherine" (Own source).

Gas exchange parameters

Net photosynthesis $\left(\mathrm{P}_{\mathrm{n}}\right)$ and stomatal conductance $\left(\mathrm{g}_{\mathrm{s}}\right)$ were measured at solar midday, in one fully expanded sun-facing leaf in the four central trees of each experimental plot per treatment, in the same days that stem water potential was 
recorded, using a field-portable photosynthesis system (LI-6400, Li-Cor, Lincoln, NE, USA).

\section{Fruit growth and yield}

Fruit diameter was measured perpendicularly to the fruit suture on 160 fruits per treatment (40 fruits per replication) every 7-10 days. Each date, 10 fruits per tree were randomly selected in the four inner trees per experimental plot and their diameters measured using a digital caliper (Powerfix model Nr Z22855F, Milomex Ltd, Bedfordshire, UK).

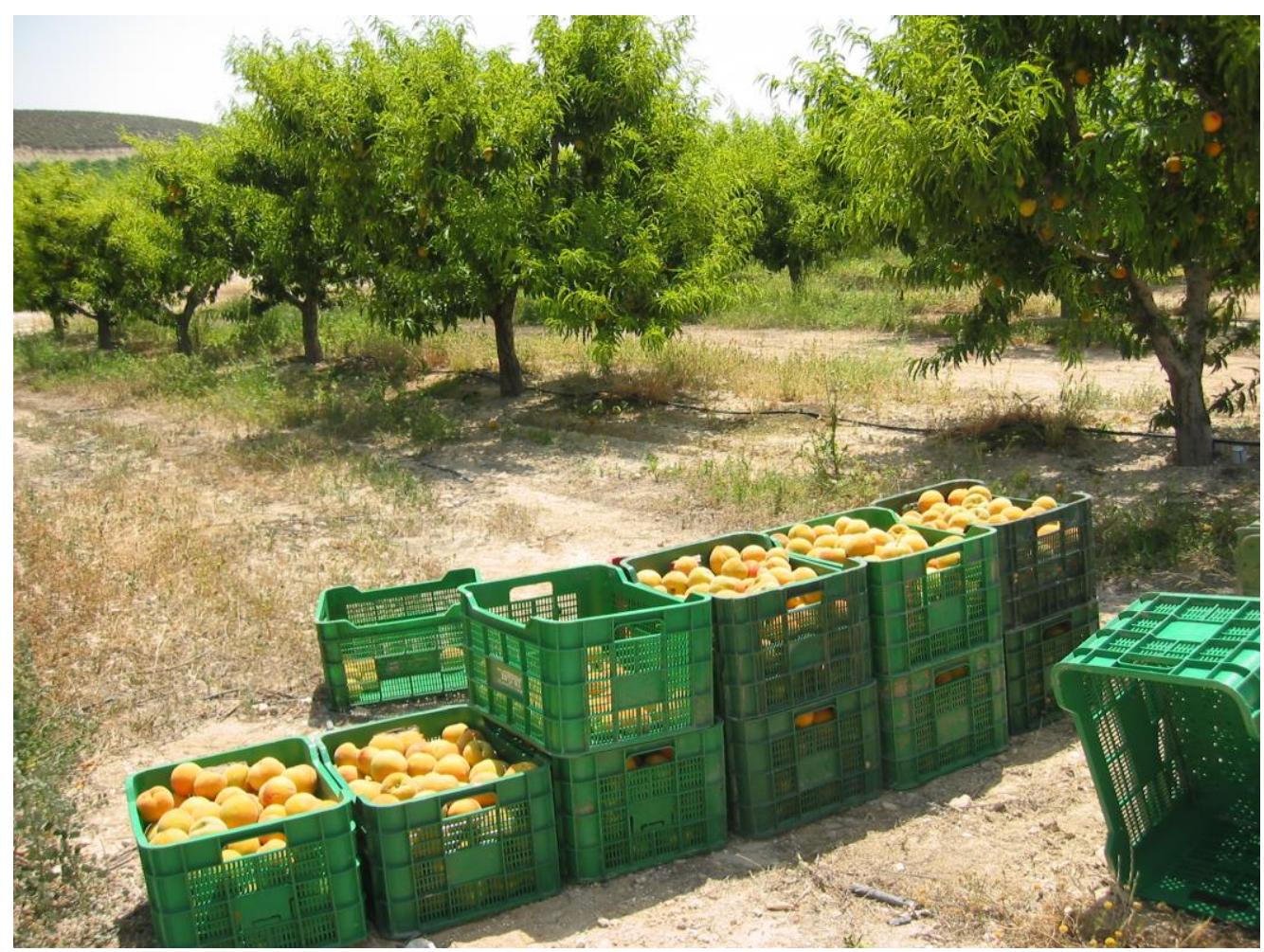

Picture 6.3. Peach harvesting (Own source).

Fruits from each tree were individually harvested on July in two or three commercial picks, depending on market demands. The number of fruits per tree was obtained by counting the fruits in the four inner trees (16 trees per treatment). The effect of the irrigation treatments on fruit size was studied evaluating marketable and nonmarketable production, as the proportion of fruits with a diameter above or below 56 mm, respectively. According to Commission Regulation (EC) $\mathrm{n}^{\circ} 1861 / 2004,56 \mathrm{~mm}$ is the minimum diameter for a fruit to be considered in the "extra" category, which can be subdivided into different categories: AAAA (diameter > $90 \mathrm{~mm})$, AAA (90 mm > diameter $\geq 80 \mathrm{~mm})$, AA $(80 \mathrm{~mm}>$ diameter $\geq 73 \mathrm{~mm})$, A $(73 \mathrm{~mm}>$ diameter $\geq 67 \mathrm{~mm})$, 
B $(67 \mathrm{~mm}>$ diameter $\geq 61 \mathrm{~mm}), \mathrm{C}(61 \mathrm{~mm}>$ diameter $\geq 56 \mathrm{~mm})$ and out of "extra" category $\mathrm{D}(56 \mathrm{~mm}>$ diameter $\geq 51 \mathrm{~mm})$.

Vegetative growth

Pruning weight was recorded for 16 trees per treatment (4 per replication) at winter pruning.

\section{Fruit quality}

At harvest, 100 fruits per treatment (25 fruits per experimental plot) were randomly selected for quality assessment. Firmness, pH, soluble solids content (SSC) and titratable acidity (TA) were evaluated as quality indices. Chemical analyses were conducted according to Artés et al. (1993). Fruit firmness was evaluated using a Durofel penetrometer DFT100 (Agro-Technologie S.A., Paris, France). Juice was extracted from combined samples of longitudinal unpeeled slices. Total soluble solids concentration (SSC) was determined with a hand refractometer (Atago, Co., Japan); values were expressed as ${ }^{\circ}$ Brix. Titratable acidity (TA) was measured by titration of $5 \mathrm{~mL}$ of juice with $0.1 \mathrm{~mol} \mathrm{~L}^{-1} \mathrm{NaOH}$ to $\mathrm{pH} 8.1$ using an automatic titration system and expressed as g malic acid $\mathrm{L}^{-1}$ (AOAC, 1984). The maturity index (MI), which affects the perception of taste (sweetness and acidity), was calculated as the ratio of SSC to TA. pH was measured using a pH-meter (Crison, Barcelona, Spain).

\section{Water stress integral}

The water stress integral (MPa-days) was calculated from the $\Psi_{\mathrm{s}}$ data in order to evaluate the intensity of water stress, according to the following equation (Myers, 1988):

$$
S_{\Psi}=\left|\sum_{i=0}^{i=t}\left(\bar{\Psi}_{i, i+1}-c\right) n\right|
$$

where:

$\Psi_{\mathrm{i}, \mathrm{i}+1}$ is the average $\Psi_{\mathrm{s}}$ for each time interval

$c$ is the value of the maximum $\Psi_{\mathrm{s}}$ in all seasons

$n$ is the number of days in the interval. 


\section{Statistical analysis}

Statistical analysis was performed as a weighted analysis of variance (ANOVA; statistical software IBM SPSS Statistics v. 21 for Windows). The Shapiro-Wilk test was used to evaluate the normality of the data. Tukey's HSD test was used for mean separation. Percentage values of fruit categories were arcsine-transformed before statistical analysis. Unless otherwise stated, the significance level was $p \leq 0.05$.

\subsection{Results}

The average values of $\mathrm{ET}_{0}$ and rainfall recorded during the experimental period were, respectively, 792 and $254 \mathrm{~mm}$ in 2008; 613 and $304 \mathrm{~mm}$ in 2009, and 784 and $188 \mathrm{~mm}$ in 2010.

Table 6.1. Average volumetric soil water content $\left(\mathrm{m}^{3} \mathrm{~m}^{-3}\right)$ for the three irrigation treatments considered at different phenological periods for the three studied growing seasons. Each data is the mean of all values in the corresponding stage. Different letters in each column indicate significant differences among treatments according to Tukey's multiple range test $(p<0.05)$.

\begin{tabular}{ccccccc}
\hline Treatment & Dormancy & $\begin{array}{c}\text { Flowering } \\
\text { and fruit set }\end{array}$ & Stage I & Stage II & Stage III & Post-Harvest \\
\hline $2008-2010$ & & & & & & \\
CETc $_{\text {ET }}$ & $0.25 \mathrm{a}$ & $0.26 \mathrm{a}$ & $0.26 \mathrm{a}$ & $0.25 \mathrm{a}$ & $0.25 \mathrm{a}$ & $0.26 \mathrm{a}$ \\
CMDS & $0.25 \mathrm{a}$ & $0.25 \mathrm{a}$ & $0.24 \mathrm{a}$ & $0.24 \mathrm{a}$ & $0.24 \mathrm{a}$ & $0.24 \mathrm{a}$ \\
RDI & $0.25 \mathrm{a}$ & $0.25 \mathrm{a}$ & $\mathbf{0 . 2 4 a}$ & $\mathbf{0 . 1 6 b}$ & $\mathbf{0 . 2 4 a}$ & $0.19 \mathrm{~b}$ \\
\hline
\end{tabular}

Table 6.1 shows the average values of the volumetric soil water content $\left(\theta_{\mathrm{v}}\right)$ recorded during the experimental period within the soil profile, from 0 to $1 \mathrm{~m}$ depth. This content was nearly constant in the $\mathrm{C}_{\mathrm{ETc}}$ and $\mathrm{C}_{\mathrm{MDS}}$ treatments, with values close to field capacity $\left(0.25 \mathrm{~m}^{3} \mathrm{~m}^{-3}\right)$. In the RDI treatment, $\theta_{\mathrm{v}}$ decreased significantly in all phenological periods when water stress was imposed. The soil moisture profile in all treatments was characterized by the fact that, during the water deficit periods, the $\theta_{\mathrm{v}}$ values at depths greater than $0.6 \mathrm{~m}$ were clearly below field capacity, indicating the non existence of drainage (data not shown).

The amounts of water applied during the 2008 growing season were 962, 727 and $413 \mathrm{~mm}$ in $\mathrm{C}_{\mathrm{ETc}}, \mathrm{C}_{\mathrm{MDS}}$ and RDI treatments, respectively. In 2009, these amounts were 849,737 and $280 \mathrm{~mm}$, whereas in 2010 they were 710, 636 and $224 \mathrm{~mm}$ (Figure $3 \mathrm{~A})$. 


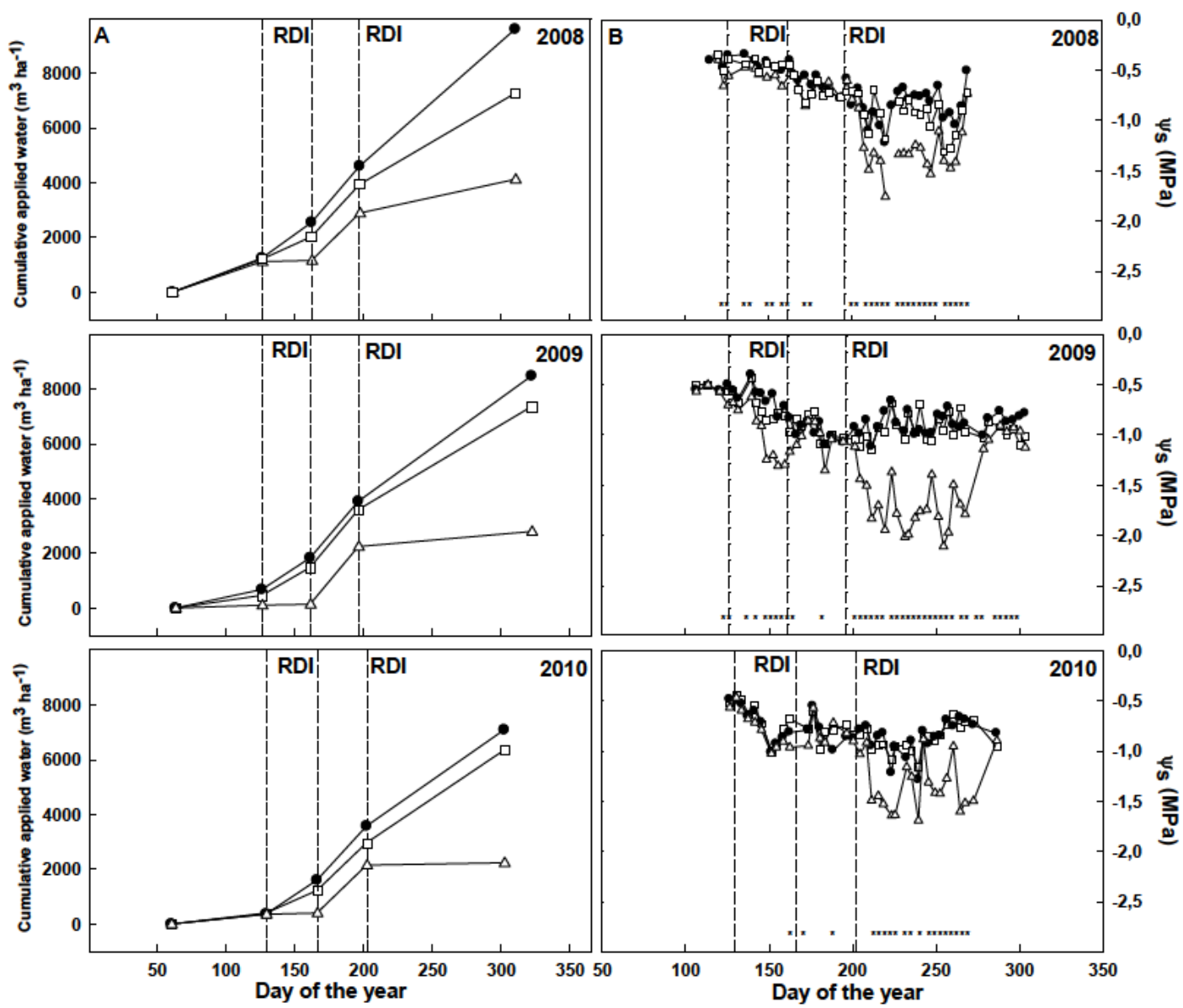

Figure 6.3. Seasonal patterns of (A) cumulative applied water and (B) midday stem water potential values for each irrigation treatment for the 3 years of the experiment: $\mathrm{C}_{\mathrm{ETc}}$ (closed circles), $\mathrm{C}_{\mathrm{MDS}}$ (open squares) and RDI (open triangles) plants. Each data point is the mean of sixteen values. Asterisks indicate significant differences among treatments $(p<0.05)$. The interval between vertical lines, from left to right, represents the beginning of stages II and III of fruit growth and postharvest.

The $\Psi_{\mathrm{s}}$ values for control trees, in average for the three growing seasons studied, decreased from an initial value of $-0.5 \mathrm{MPa}$ (stage I) to $-1.0 \mathrm{MPa}$ (post-harvest). During 2008 , significant differences among treatments were observed for $\Psi_{\mathrm{s}}$ in stage II, and these were more clearly observed in 2009 , whereas not significant differences were detected for this stage in 2010 (Figure 6.3B). After harvest, more negative $\Psi_{\mathrm{s}}$ values were evident for the RDI treatment about 10-15 days after water restriction started.

These differences increased, reaching average $\Psi_{\mathrm{s}}$ values of -1.24 MPa for RDI in $2008,-1.52 \mathrm{MPa}$ in 2009 and $-1.36 \mathrm{MPa}$ in 2010. Under the $\mathrm{C}_{\mathrm{MDs}}$ treatment, similar $\Psi_{\mathrm{s}}$ values to those of the $\mathrm{C}_{\mathrm{ETc}}$ were observed until the end of the growing season. When full irrigation was restored, all treatments showed similar $\Psi_{\mathrm{s}}$ values. 


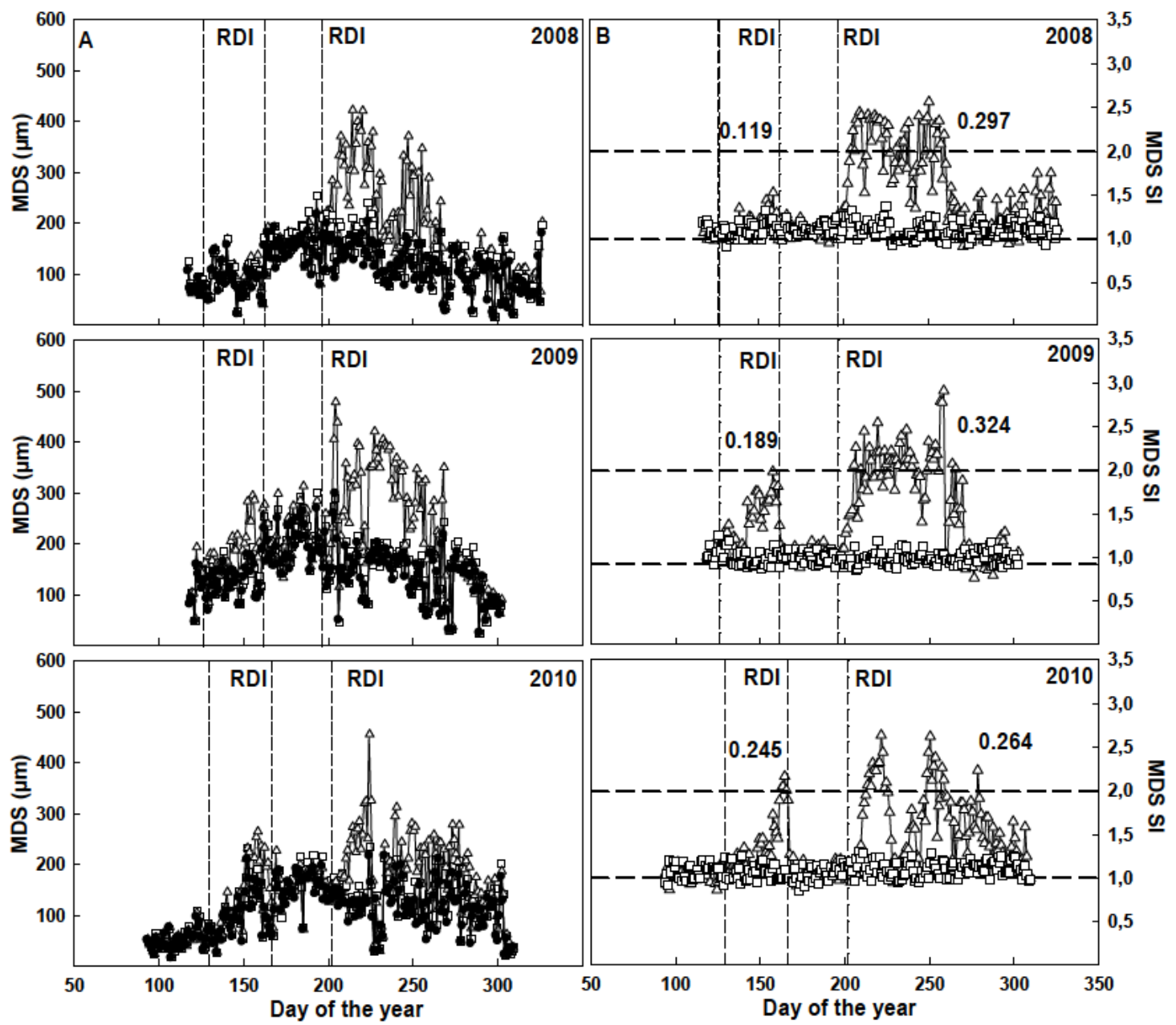

Figure 6.4. Maximum daily shrinkage $(A, \mu \mathrm{m})$ evolution in the $\mathrm{C}_{\mathrm{ETc}}$ (closed circles), $\mathrm{C}_{\mathrm{MDS}}$ (open squares) and RDI (open triangles) treatments, and maximum daily shrinkage signal intensity (MDS $\mathrm{SI})$ (B) as $\mathrm{C}_{\mathrm{MDS}} / \mathrm{C}_{\mathrm{ETc}}$ ratio (open squares) and $\mathrm{RDI} / \mathrm{C}_{\mathrm{ETc}}$ ratio (open triangles) for the irrigation treatments during the fruit growth period 2008-2010. The interval between vertical short dash lines from left to right represent the beginning of stages II and III of fruit growth and post-harvest. Horizontal short dash lines represent the threshold values for MDS SI. Each data point is the mean of four values.

The MDS evolution over the growing season (Figure 6.4A) was similar for the $\mathrm{C}_{\mathrm{ETc}}$ and $\mathrm{C}_{\mathrm{MDS}}$ treatments, with increasing values in the summer months. In RDI plants, MDS values showed a tendency to increase because of the imposed reduced irrigation volumes. Just a few days after the onset of the irrigation deficit period, the daily values of MDS of the plants under RDI treatment showed a significant increase, reaching values of $400 \mu \mathrm{m}$. These values of MDS resulted in MDS SI levels close to the threshold values established for both treatments. For the $\mathrm{C}_{\mathrm{MDS}}$ treatment, MDS SI values were close to unity all over the three-year period. For the RDI treatment, MDS SI only reached threshold values in the last part of stage II in 2009 and 2010 and in postharvest for the three years. 
The coefficient of variation (CV) of MDS SI throughout the growing season in the $\mathrm{C}_{\mathrm{MDS}}$ treatment was around 0.080 , very close to the values obtained in the RDI treatment during stage III of fruit growth (0.078), indicating that imposed irrigation was similar. The CV values increased during stage II of fruit growth, with the lowest value (0.119) in 2008 when water stress conditions were not reached, and increasing during 2009 and 2010 in proportion to the water stress level reached. During the postharvest period, the $\mathrm{CV}$ was similar in the three years, with values ranging from 0.264 to 0.324 .

Overall for the whole season, $\mathrm{S}_{\Psi}$ values reflected significant differences among treatments, RDI showed the highest values of water stress integral (Figure 6.5), which were significantly higher than those from $\mathrm{C}_{\mathrm{ETc}}$ and $\mathrm{C}_{\mathrm{MDS}}$ in the three years of the experiment. No significant differences were observed between $\mathrm{C}_{\mathrm{ETc}}$ and $\mathrm{C}_{\mathrm{MDS}}$ treatments.

\begin{tabular}{|c|c|c|c|c|}
\hline & Stage I & Stage II & Stage III & Post-Harvest \\
\hline \multicolumn{5}{|l|}{2008} \\
\hline $\mathrm{C}_{\text {Mds }} / \mathrm{C}_{\mathrm{ETc}}$ & $1.10 a$ & $1.09 a$ & $1.11 \mathrm{a}$ & $1.10 a$ \\
\hline $\begin{array}{c}\text { RDI/C } \\
2009\end{array}$ & $1.04 a$ & $1.15 a$ & $1.09 a$ & $1.95 b$ \\
\hline CMds/Cetc & $1.13 a$ & $1.06 a$ & $1.08 \mathrm{a}$ & $1.06 a$ \\
\hline $\begin{array}{c}\text { RDI/CETc } \\
2010\end{array}$ & $1.14 a$ & $1.48 b$ & $1.11 \mathrm{a}$ & $2.03 b$ \\
\hline $\mathrm{C}_{\text {Mds }} / \mathrm{C}_{\mathrm{ETc}}$ & $1.09 a$ & $1.07 a$ & $1.06 a$ & $1.11 a$ \\
\hline RDI/CETc & $1.03 a$ & $1.37 b$ & $1.05 a$ & $1.61 b$ \\
\hline
\end{tabular}

Table 6.2 shows the average values of MDS SI for the different growth stages during each year studied. The MDS SI values were higher for the RDI treatment in all stages during which the water deficit was imposed, except for stage II in 2008 when rainfall events and mild temperatures occurred. The $\mathrm{C}_{\mathrm{MDS}}$ treatment showed average MDS SI values close to unity throughout the growing season in the three years studied.

Significant differences between RDI and $\mathrm{C}_{\mathrm{ETc}}$ treatments were detected for pruning weight (Figure 6.6). In 2009, these differences were also significant with 
respect to $\mathrm{C}_{\mathrm{MDS}}$. However, no significant effects have been observed on the evolution of fruit equatorial diameter for the irrigation treatments studied $\left(\mathrm{C}_{\mathrm{ETc}}, \mathrm{C}_{\mathrm{MDS}}\right.$ and RDI) (data not shown).

Crop load and yield were similar among treatments, although highly variable between years (Table 6.3). Furthermore, no differences among treatments were observed for fruit distribution into commercial categories (Figure 6.7).

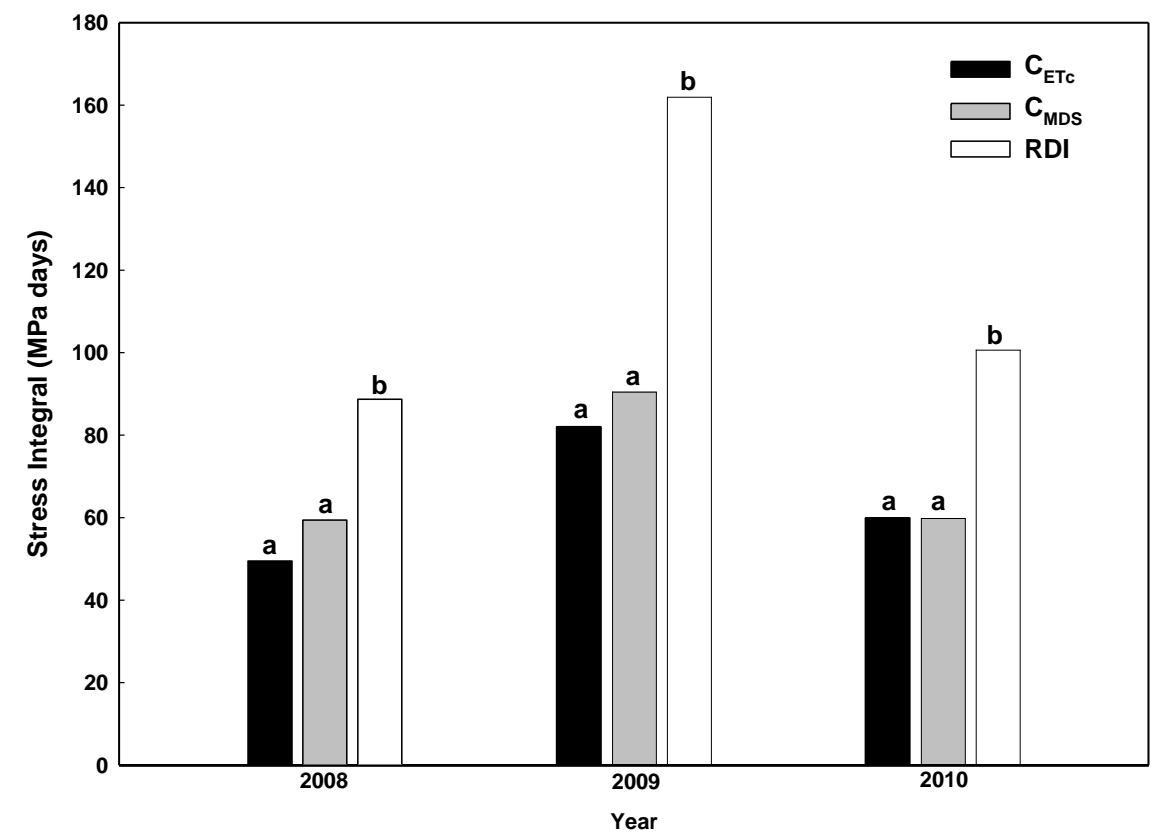

Figure 6.5. Water stress integral for each irrigation treatment, $\mathrm{C}_{\mathrm{ETc}}$ (black bars), $\mathrm{C}_{\mathrm{MDS}}$ (gray bars) and RDI (white bars) in the 2008, 2009 and 2010 growing seasons. Different letters on top of bars indicate significant differences according to Tukey's multiple range test $(p<0.05)$.

The fact that similar yields were obtained for the three treatments studied made that water productivity (WP) values were higher under the RDI treatment when compared to $\mathrm{C}_{\mathrm{ETc}}$ and $\mathrm{C}_{\mathrm{MDS}}$ (Table 6.3), although they were similar between $\mathrm{C}_{\mathrm{ETc}}$ and $\mathrm{C}_{\text {MDs. }}$. Consequently, important water savings were registered for the RDI treatment. Depending of the year, RDI supposed between 43 and $65 \%$ water savings for the whole season (Table 6.3). 


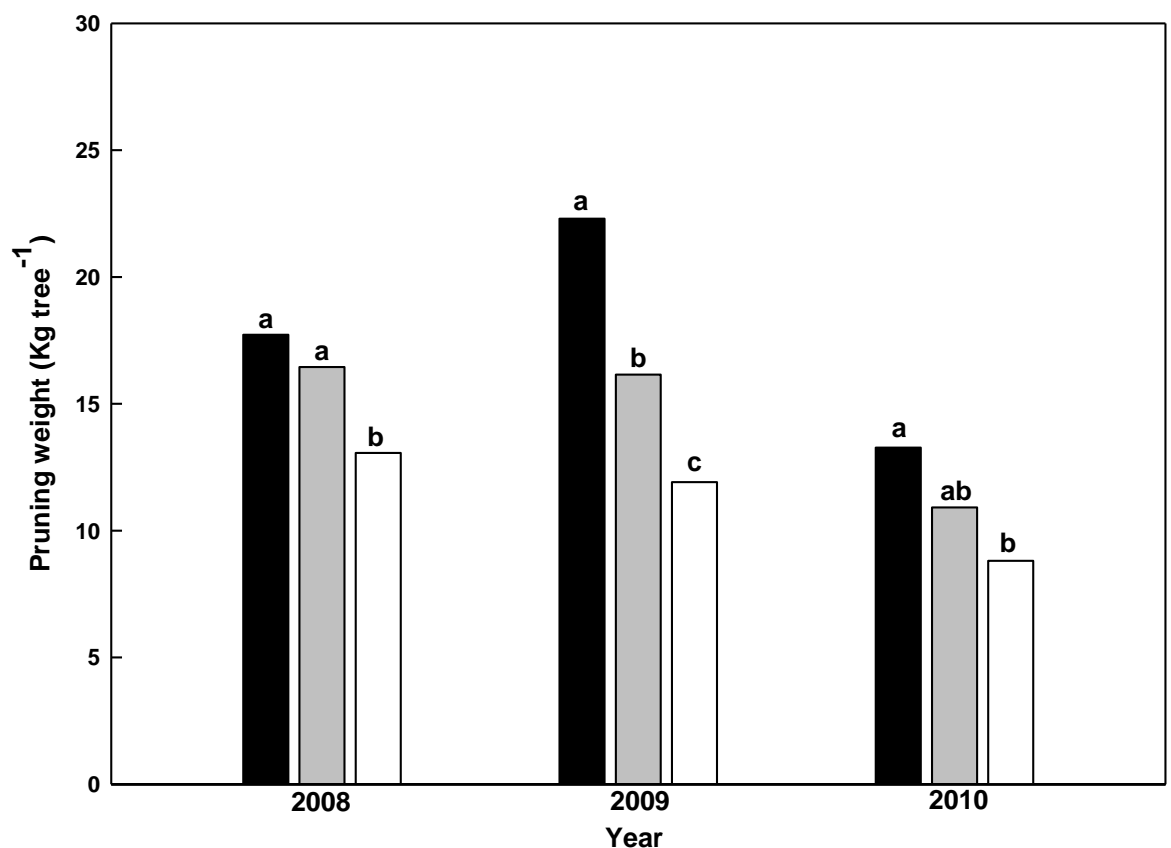

Figure 6.6. Pruning weight as a function of the irrigation treatment, $\mathrm{C}_{\mathrm{ETc}}$ (black bars), $\mathrm{C}_{\mathrm{MDS}}$ (gray bars) and RDI (white bars) in the 2008, 2009 and 2010 growing seasons. Each bar corresponds to the mean of sixteen trees. Different letters on top of bars indicate significant differences according to Tukey's multiple range test ( $p$ $<0.05)$.

Table 6.3. Crop load, yield, water productivity (WP) and water spends/savings in each non-critical period and total in response to the different irrigation treatments for the experimental period 2008-

2010. Different letters in the column within each year indicate significant differences among treatments according to Tukey's multiple range test $(p<0.05)$.

\begin{tabular}{|c|c|c|c|c|c|c|}
\hline & \multirow{2}{*}{$\begin{array}{c}\text { Crop load } \\
\left.\text { (Fruits tree }^{-1}\right) \\
\end{array}$} & \multirow{2}{*}{$\begin{array}{c}\text { Yield } \\
\left(\mathrm{Kg} \mathrm{tree}^{-1}\right) \\
\end{array}$} & \multirow{2}{*}{$\begin{array}{c}\mathrm{WP} \\
\left(\mathrm{Kg} \mathrm{m}^{-3}\right) \\
\end{array}$} & \multicolumn{3}{|c|}{ Water spends/savings (\%) } \\
\hline & & & & SII & Postharvest & Total \\
\hline \multicolumn{7}{|l|}{2008} \\
\hline $\mathbf{C}_{\text {ETc }}$ & 170a & 29.87a & $1.29 \mathrm{a}$ & & & \\
\hline $\mathrm{C}_{\text {MdS }}$ & $150 a$ & 24.74a & $1.42 \mathrm{a}$ & & & \\
\hline RDI & $127 \mathbf{a}$ & 21.18a & $2.07 b$ & 95 & 37 & 43 \\
\hline \multicolumn{7}{|l|}{2009} \\
\hline $\mathbf{C}_{\text {ETc }}$ & 489a & $67.23 a$ & $3.30 \mathrm{a}$ & & & \\
\hline $\mathrm{C}_{\mathrm{MDS}}$ & $487 a$ & $60.75 a$ & $3.43 \mathrm{a}$ & & & \\
\hline RDI & $467 a$ & 63.13a & $9.39 \mathrm{~b}$ & 97 & 85 & 62 \\
\hline \multicolumn{7}{|l|}{2010} \\
\hline CETc $_{\text {ETc }}$ & 173a & $30.16 a$ & $1.77 \mathbf{a}$ & & & \\
\hline $\mathrm{C}_{\text {MDS }}$ & 153a & $26.02 a$ & $1.70 a$ & & & \\
\hline RDI & 181a & 32.13a & $5.97 b$ & 95 & 97 & 65 \\
\hline
\end{tabular}

In relation to fruit quality (Table 6.4), no significant differences were detected for firmness. Significant differences in pH were detected in 2008 and 2009. Soluble solids content (SSC) was higher in the fruits from the RDI treatment in 2009 and 2010. Titratable acidity (TA) was lower in the RDI treatment in 2009 and 2010. Consequently, 
the maturity index or ratio SSC/TA presented higher values in the RDI treatment in both years (2009 and 2010).
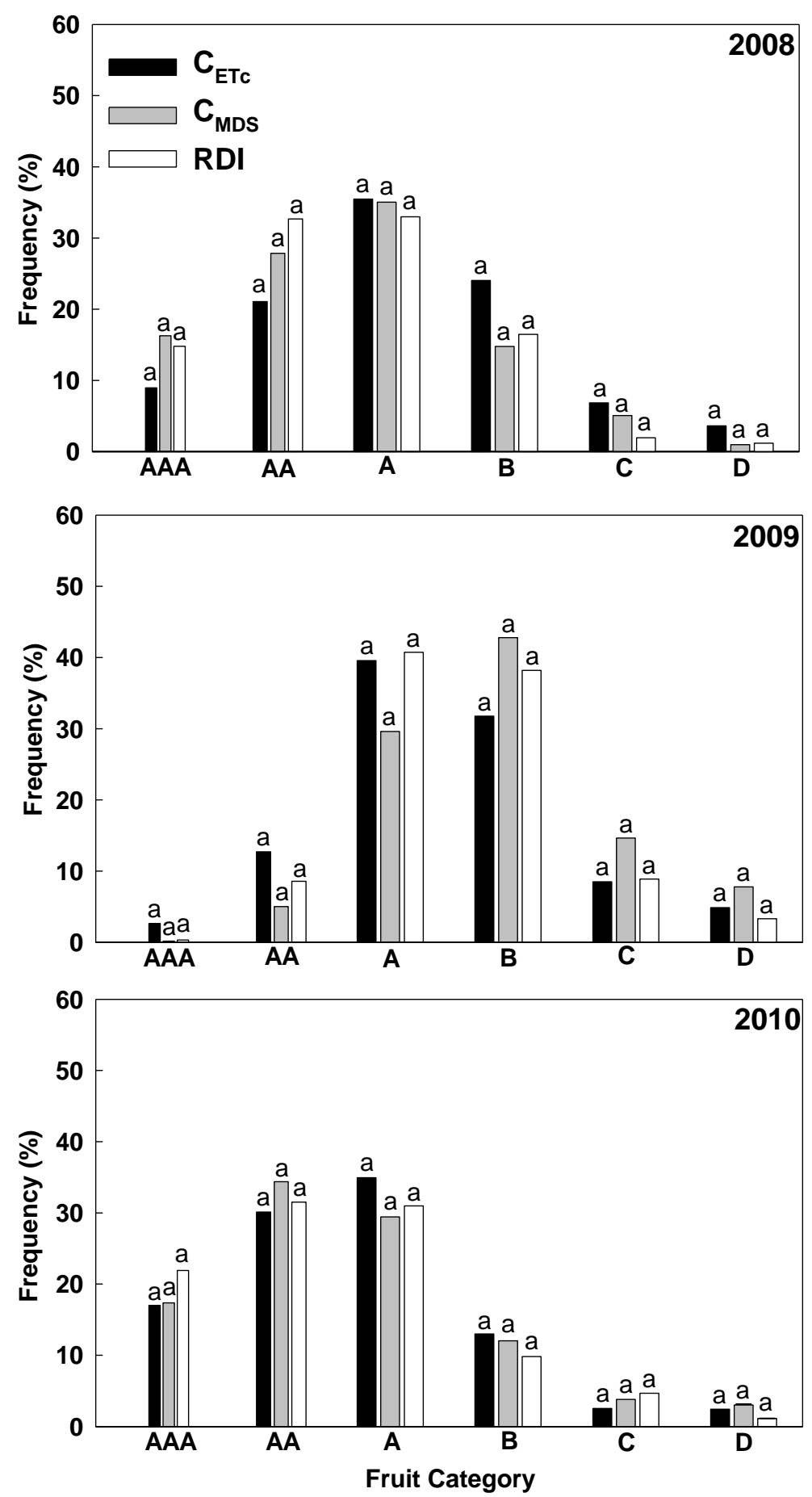

Figure 6.7. Percentage of fruit categories for each irrigation treatment, $\mathrm{C}_{\mathrm{ETc}}$ (black bars), $\mathrm{C}_{\mathrm{MDS}}$ (gray bars) and RDI (white bars) in the 2008, 2009 and 2010 growing seasons. Each bar corresponds to the mean of sixteen trees. Different letters on top of bars indicate significant differences according to Tukey's multiple range test $(p<0.05)$. 
Table 6.4. Fruit firmness, pH, soluble solids content (SSC), tritratable acidity (TA) and SSC/TA ratio at harvest for all treatments. Values are the mean of 100 measurements. Different letters in each column within each year indicate significant differences according to Tukey's multiple range test $(p<0.05)$.

\begin{tabular}{|c|c|c|c|c|c|}
\hline & $\begin{array}{l}\text { Firmness } \\
(\mathbf{N})\end{array}$ & $\begin{array}{c}\text { pH } \\
-\end{array}$ & $\begin{array}{c}\text { SSC } \\
\left({ }^{\circ} \text { Brix }\right)\end{array}$ & $\begin{array}{c}\text { TA } \\
\left(\mathrm{g} 100 \mathrm{~mL}^{-1}\right)\end{array}$ & $\begin{array}{c}\text { Maturity Index } \\
\text { (SSC/TA) }\end{array}$ \\
\hline \multicolumn{6}{|l|}{2008} \\
\hline $\mathrm{C}_{\text {ETc }}$ & $50.47 \mathrm{a}$ & 4.13a & $10.64 a$ & $0.59 a$ & 18.82a \\
\hline $\mathrm{C}_{\text {MDS }}$ & $54.10 \mathrm{a}$ & $3.99 \mathrm{~b}$ & $10.62 \mathrm{a}$ & $0.60 a$ & 19.49a \\
\hline RDI & $53.90 \mathrm{a}$ & $4.00 \mathrm{~b}$ & $10.06 a$ & $0.51 \mathrm{a}$ & $20.35 a$ \\
\hline \multicolumn{6}{|l|}{2009} \\
\hline CETc & 49.29a & $3.86 a$ & $10.61 \mathrm{a}$ & $0.61 \mathrm{a}$ & $17.91 \mathrm{a}$ \\
\hline$C_{\text {Mds }}$ & 49.39a & $3.83 a$ & $10.83 a$ & $0.66 a$ & $17.43 \mathrm{a}$ \\
\hline RDI & 53.61a & $4.00 \mathrm{~b}$ & $11.21 \mathrm{~b}$ & $0.52 b$ & $21.86 \mathrm{~b}$ \\
\hline \multicolumn{6}{|l|}{2010} \\
\hline CETc $_{\text {ET }}$ & $52.23 a$ & 3.88a & $11.51 \mathrm{a}$ & $0.75 a$ & $15.60 \mathrm{a}$ \\
\hline $\mathrm{C}_{\text {MDS }}$ & $52.82 \mathrm{a}$ & $3.86 a$ & $11.66 a$ & $0.75 a$ & $15.86 \mathrm{a}$ \\
\hline RDI & $52.14 \mathrm{a}$ & $3.83 \mathrm{a}$ & $12.25 \mathrm{~b}$ & $0.67 b$ & $18.73 b$ \\
\hline
\end{tabular}

\subsection{Discussion}

The results from the current study confirmed the hypothesis that it is possible to schedule irrigation in mid-late maturing peach trees by keeping MDS SI values close to unity and also to develop a moderate water deficit maintaining the values of MDS SI close to 2 in the RDI treatment during non-critical periods (stage II of fruit development and postharvest), saving a considerable amount of water without affecting yield and fruit quality. This observation is in accordance with other studies that have shown how maintaining MDS signal intensity around the unity could be successfully employed for scheduling irrigation in other fruit species such as lemon, early-maturing peach or pomegranate trees (e.g. Conejero et al., 2007 and 2010; Ortuño et al., 2009; Intrigliolo et al., 2011).

In our experiment, $\theta_{\mathrm{v}}$ in $\mathrm{C}_{\mathrm{ETc}}$ remained close to field capacity during the 3 years of the experimental period indicating that irrigation scheduling in this treatment kept the plants under non-limiting soil water conditions (Table 6.1). Similarly, trees from the $\mathrm{C}_{\mathrm{MDS}}$ treatment did not suffer from water restrictions. The deficit irrigation (RDI) during stage II and postharvest resulted in a reduction in $\theta_{\mathrm{v}}$ values, which induced a decrease in $\Psi_{\mathrm{s}}$ values, reflecting a moderate drought stress especially during the postharvest period (Figure 6.3B). In addition, these conditions induced an increase in 
MDS and MDS SI values (Figure 6.4), proving that these signal intensities can be a useful tool for irrigation scheduling.

The fact that MDS SI values in the $\mathrm{C}_{\text {MDS }}$ treatment showed a low variability and remained close to the selected MDS SI threshold value (unity) and that their $\Psi_{\mathrm{s}}$ values and gas exchange measurements (data not shown) were similar to those of $\mathrm{C}_{\mathrm{ETc}}$ plants shows that the irrigation water amounts applied were suitably adjusted and able to satisfy plant water requirements. This would allow us to estimate crop coefficient values for this species when these are not available or to adjust the requirements according to the area where trees are located.

In addition, MDS SI in the RDI treatment showed more variability during the stage II and postharvest periods than in the other treatments, values increased between 0.119 (stage II 2008) and 0.324 (postharvest 2009). This indicated that the information generated from these records under RDI was sufficient to adjust the irrigation schedule to maintain MDS signal intensity close the selected threshold values and that peach tree irrigation scheduling can be exclusively based on MDS measurements (Conejero et al., 2007; Ortuño et al., 2009). In the RDI treatment, the MDS SI threshold was only reached during the stage II at the end of this phase in 2009 and 2010, showing these the highest values of $\mathrm{CV}$. However, this threshold was reached during the 3 years in the postharvest period, in a similar way a similar $\mathrm{CV}$ was observed between years, about 0.3 , being the highest one (0.324) reached in 2009 , coinciding with the greatest water stress integral observed over the study period.

Goldhamer and Fereres (2004) considered, in almond trees, that a threshold value of 1.75 would result in mild water stress with little effect on production, and that a threshold value of 2.75 would cause a more severe water stress. The severe treatment (MDS SI 2.75) caused hastening fruit maturation (hull splitting), while the less severe deficit irrigation regime (MDS SI 1.75) did not affect kernel size and other important almond fruit parameters. Conejero et al. (2007), in peach trees, followed an irrigation scheduling based on maintaining MDS SI threshold value close to 1 . Their results indicated the consistence and sensibility of this parameter to changes in peach tree water status. García-Orellana et al. (2007) followed the same approach for lemon trees, using two levels of the MDS SI fixed to threshold values of 1.25 and 1.35. The authors concluded that it is feasible to schedule irrigation in lemon trees using only MDS SI 
values. Vélez et al. (2007) reached water savings between 12-18\% from June to October, maintaining the threshold value of MDS SI close to 1.25 in "Clementina de Nules" trees. These results suggest that deciduous trees accepted higher threshold values of MDS SI respect to perennial tress.

Pruning weight was significantly reduced by irrigation withholding during stage II and postharvest, when compared to full irrigation conditions, as previously reported in similar studies (López et al., 2008; Abrisqueta et al., 2010) (Figure 6.6). This reduction seems to be directly related to the amount of water applied and is desirable for high-density orchards in order to optimize tree-light interception and improve economic revenues (Chalmers et al., 1981) due to lower operational costs.

In contrast, fruit growth was not reduced by water deprivation during stage II and postharvest showing the typical double sigmoidal curve characteristic of stone fruit growth (Baldini, 1992) (data not shown). In fact, similar crop loads and yields were observed for $\mathrm{C}_{\mathrm{MDS}}$ and RDI treatments when compared to $\mathrm{C}_{\mathrm{ETc}}$ trees (Table 6.3). This trend was maintained over the three years of the experiment. Therefore, greater water productivity values were observed for the RDI treatments.

Figure 6.7 represents the percentage of fruit categories, a higher percentage of fruits within the largest sizes (AAA and AA) were observed in 2009 compared to the other two years. This was caused by a lesser thinning in this year than in 2008 and 2010, because the fate of fruits was not fresh consumption on that year, as corroborated by the load and yield (Table 6.3).

Fruit quality indices such as SSC and TA warrant particular attention due to their importance in fruit taste (Crisosto et al., 1994). In our case, SSC values were increased in 2009 and 2010 in RDI treatment (Table 6.4). Higher values of the maturity index were observed during 2009 and 2010, being significantly higher under RDI. This ratio affects the perception of taste (sweetness and acidity) by the consumer, thereby influencing buying decisions (Crisosto et al., 1997).

In summary, this study demonstrated that irrigation scheduling in peach trees can be based exclusively on the MDS SI measures was feasible without detrimental effects on yield and fruit quality. In this sense, water savings of $43-65 \%$ and water 
productivity increments in the RDI treatment were achieved without affecting production and improving fruit quality.

This study also showed the possibility to quantify peach tree water requirements maintaining MDS SI values close to unity during crop growth, in order to determine crop factor $\left(\mathrm{K}_{\mathrm{c}}\right)$ for this species.

\section{Acknowledgements}

This study was supported by IRRIQUAL (EU-FP6-FOOD-CT-2006-023120) and SIRRIMED (KBBE-2009-1-2-03, PROPOSAL N॰245159) projects. We are also grateful to two SENECA projects (05665/PI/07 and 11872/PI/09) and CONSOLIDER INGENIO 2010 (MEC CSD2006-0067) and CICYT (AGL2010-17553) projects for providing funds to finance this research.

\subsection{References}

Abrisqueta, I., Tapia, L.M., Conejero, W., Sánchez-Tobirio, M.I., Abrisqueta, J.M., Vera, J., Ruiz-Sánchez, M.C., 2010. Response of early-peach [Prunus persica (L.)] trees to deficit irrigation. Span. J. Agric. Res. 8(S2), S30-S39.

Allen, R.G., Pereira, L.S., Raes, D., Smith, M., 1998. Crop evapotranspirationguidelines for computing crop water requirements. Irrigation and Drainage No. 56, FAO, Roma. 300 pp.

AOAC, 1984. Association of Official Analytical Chemists. Official methods of analysis, 15th edn. AOAC, Washington DC.

Artés, F, Escriche, A.J, Martínez, J.A., Marín, J.G, 1993. Quality factors in four varieties of melon (Cucumis melo, L.). J Food Quality 16:91-100.

Baldini, E. 1992. Arboricultura General. Mundi-Prensa Ed. Madrid. 379 pp.

Chalmers, D.J., Mitchell, P.D., Van Heek, L., 1981. Control of peach tree growth and productivity by regulated water supply, tree density and summer pruning. J. Am. Soc. Hort. Sci. 106, 307-312.

Crisosto, C.H., Johnson, R.S., Luza, J.G., Crisosto, G.M., 1994. Irrigation regimes affect fruit soluble solids concentration and rate of water loss of "O'Henry" peaches. Hort Science 29, 1169-1171.

Crisosto, C.H., Johnson, R.S., Dejong, T., Day, K.R., 1997. Orchard factors affecting postharvest stone-fruit quality. Hort Science 32, 820-823.

Conejero, W., Alarcón, J.J., García-Orellana, Y., Abrisqueta, J.M., Torrecillas, A., 2007. Daily sap flow and maximum daily trunk shrinkage measurements for diagnosing water stress in early maturing peach trees during the post-harvest period. Tree Physiol. 27, 81-88. 
Conejero, W., Mellisho, C.D., Ortuño, M.F., Galindo, A., Perez-Sarmiento, F., Torrecillas, A., 2010. Establishing maximum daily trunk shrinkage and midday stem water potential reference equations for irrigation scheduling of early maturing peach trees. Irrigation Science 29, 299-309.

Conejero, W., Mellisho, C.D., Ortuño, M.F., Moriana, A., Moreno, F., Torrecillas, A., 2011. Using trunk diameter sensors for regulated deficit irrigation scheduling in early maturing peach trees. Environmental and Experimental Botany 71, 409415.

De la Rosa, J.M., Conesa, M.R., Domingo, R., Pérez-Pastor, A., 2014. A new approach to ascertain the sensitivity to water stress of different plant water indicators in extra-early nectarine trees.Scientia Horticulturae 169 (2014) 147-153.

Fernández, J.E., Cuevas, M.V., 2010. Irrigation scheduling from stem diameter variations: A review. Agric. For. Meteorol. 150, 135-151.

Fereres, E., Soriano, M.A., 2007. Déficit irrigation for reducing agricultural water use. J. Exp. Bot. 58, 147-159.

García-Orellana, Y., Ruiz-Sánchez, M.C., Alarcón, J.J., Conejero, W., Ortuño, M.F., Nicolás, E., Torrecillas, A., 2007. Preliminary assessment of the feasibility of using maximum daily trunk shrinkage for irrigation scheduling in lemon trees. Agric. Water Manage. 89, 167-171.

Goldhamer, D.A., Fereres, E., 2001. Irrigation scheduling protocols using continuously recorded trunk diameter measurements. Irrig. Sci. 20, 115-125.

Goldhamer, D.A., Fereres, E., 2004. Irrigation scheduling of almond trees with trunk diameter sensors. Irrig. Sci. 23, 11-19.

Intrigliolo, D.S., Puerto, H., Bonet, L., Alarcón, J.J., Nicolas, E., Bartual, J., 2011. Usefulness of trunk diameter variations as continuous water stress indicators of pomegranate (Punica granatum) trees. Agric. Water Manag. 98, 1462-1468.

Katerji, N., Tardieu, F., Bethenod, O., Quetin, P., 1994. Behaviour of maize stem diameter during drying cycles: comparison of two methods for detecting water stress. Crop Sci. 34, 165-169.

López, G., Arbonés, A., Del Campo, J., Mata, M., Vallverdú, X., Girona, J., Marsal, J., 2008. Response of peach trees to regulated deficit irrigation during stage II of fruit development and summer pruning. Span. J. Agric. Res. 6, 479-491.

MAGRAMA, 2015. Anuario de estadística Avance 2014. Ministerio de Agricultura, Alimentación y Medio Ambiente.

Moreno, F., Fernández, J.E., Clothier, B.E., Green, S.R., 1996. Transpiration and root water uptake by olive trees. Plant Soil 184, 85-96.

Myers, B.J., 1988. Water stress integral a link between short-term stress and long term growth. Tree Physiol. 4, 315-323. 
Naor, A., Cohen, S., 2003. Sensitivity and variability of maximum trunk, shrinkage, midday stem water potential, and transpiration rate in response to withholding irrigation from field-grown apple trees. HortScience 38, 547-551.

Ortuño, M.F., García-Orellana, Y., Conejero, W., Ruiz-Sánchez, M.C., Mounzer, O., Alarcón, J.J., Torrecillas, A., 2006. Relationships between climatic variables and sap flow, stem water potential and maximum daily trunk shrinkage in lemon trees. Plant Soil 279, 229-242.

Ortuño, M.F., Brito, J.J., Conejero, W., García-Orellana, Y., Torrecillas, A., 2009. Using continuously recorded trunk diameter fluctuations for estimating water requirements of lemon trees. Irrig. Sci. 27, 271-276.

Ortuño, M.F., Conejero, W., Moreno, F., Moriana, A., Intrigliolo, D.S., Biel, C., Mellisho, C.D., Pérez-Pastor, A., Domingo, R., Ruiz-Sánchez, M.C., Casadesus, J., Bonany, J., Torrecillas, A., 2010. Could trunk diameter sensors be used in woody crops for irrigation scheduling? A review of current knowledge and future perspectives. Agr. Water Manage. 97, 1-11.

Puerto, P., Domingo, R., Torres, R., Pérez-Pastor, A., García-Riquelme, M., 2013. Remote management of deficit irrigation in almond trees based on maximum daily trunk shrinkage. Water relations and yield. Agric. Water Manag 126 (2013) 33- 45 .

Turner, N.C, 1981. Techniques and experimental approaches for the measurement of plant water status. Plant Soil 58:339-366.

Vélez, J.E., Intrigliolo, D.S., Castel, J.R., 2007. Scheduling deficit irrigation of citrus trees with maximum daily trunk shrinkage. Agric. Water Manag. 90, 197-204. 


\section{USE OF MIDDAY STEM WATER POTENTIAL FOR DEFICIT IRRIGATION SCHEDULING IN PEACH ORCHARDS IN SOUTH-EAST SPAIN}





\title{
7. USE OF MIDDAY STEM WATER POTENTIAL FOR DEFICIT IRRIGATION SCHEDULING IN PEACH ORCHARDS IN SOUTH-EAST SPAIN
}

\begin{abstract}
Irrigation techniques that reduce water spends are being increasingly applied in areas with scarce water resources. In this study, the effect of two different regulated deficit irrigation (RDI) strategies on peach [Prunus persica (L.) Batsch cv. "Catherine"] performance were studied over three growing seasons. The experimental site was located in Murcia (SE Spain), a Mediterranean region. Two RDI strategies (restricting water applications at stage II of fruit development and postharvest) based on stem water potential $\left(\Psi_{\mathrm{s}}\right)$ thresholds were compared to a fully irrigated control. Soil water content $\left(\theta_{\mathrm{v}}\right), \Psi_{\mathrm{s}}$, gas exchange parameters, vegetative growth, crop load, yield and fruit quality were determined. RDI treatments showed significantly lower values of $\theta_{\mathrm{v}}$ and $\Psi_{s}$ than control trees when irrigation water was restricted, causing reductions in stomatal conductance and photosynthesis rates. Vegetative growth was reduced by RDI, as lower shoot lengths and pruning weights were observed under those treatments when compared to control. However, fruit size and yield were unaffected. Crop quality was slightly improved by RDI. Water savings up to $65 \%$ were achieved depending on the year and the RDI strategy and no negative carry-over effect was detected during the study period. In conclusion, RDI strategies using $\Psi_{\mathrm{s}}$ thresholds for scheduling irrigation in mid-late maturing peach trees under Mediterranean conditions is a viable option to save water without compromising yield.
\end{abstract}

Keywords: Regulated deficit irrigation; fruit quality; stem water potential; soil water content; Prunus persica; yield.

\subsection{Introduction}

Agriculture is the primary user of water worldwide, reaching a proportion that exceeds $70-80 \%$ of the total in arid and semi-arid regions (Fereres and Soriano, 2007). The pressure on water resources is increased by the competition for water between agriculture, industry and population. This situation leads to find new ways of reducing water use in agriculture, especially for fruit tree irrigation. 
Spain is the second largest peach [Prunus persica (L.) Bastch] producer in the world. Peach tree plantations are located in the Mediterranean area, being Murcia (SE Spain) the third main peach production area in the country, yielding 249,500 t per year, $19 \%$ of the Spanish production, approximately (MAGRAMA, 2015).

Due to the increasing limitation of irrigation water for horticultural crops in Mediterranean areas, characterized by water shortage, there is an increasing risk of losing irrigated land. Hence, reducing applied water during certain periods of the year could improve water use efficiency and conservation. Under these circumstances, the focus would not only be to achieve productions above the average or control vegetative growth but to reduce water use even at a possible risk of a slight reduction in production (Girona et al., 2003). Therefore, precise tools for assessing crop water requirements are needed in order to cope with water scarcity and reduce irrigation water use without compromising crop yield and quality.

One of the most promising techniques to achieve this objective is Regulated Deficit Irrigation (RDI), which was developed in Australian peach orchards (Chalmers et al., 1981). This technique consists of applying less irrigation water than crop evapotranspiration $\left(\mathrm{ET}_{\mathrm{c}}\right)$ during certain periods of the crop cycle when yield and quality would be hardly affected, restoring full irrigation for the rest of the cycle. The concept was to control excessive vegetative growth and enhance fruit growth (Chalmers et al., 1981), other advantages have also been described such as improvements of fruit quality and taste (Crisosto et al., 1994; López et al., 2011).

RDI has normally been applied when reproductive growth is relatively slow and when vegetative growth and other plant processes may be affected, such effects being translated into improved fruit quality (Ruiz-Sánchez et al., 2010). In this respect, RDI has been successfully employed in many fruit crops including citrus (Goldhamer and Salinas, 2000; González-Altozano and Castel, 2000), peach (Girona et al., 2003; Buendía et al., 2008; López et al., 2008), and other species (Ruiz-Sánchez et al., 2010).

In most of the research on peach responses to RDI, water restriction was applied during stages I and II of fruit development (initial growth and pit hardening, respectively), as well as at postharvest, whereas full irrigation was applied at stage III when deficit irrigation should provoke yield reductions (Abrisqueta et al., 2010). 
The water balance method (Allen et al., 1998) is the established technique for estimating full irrigation requirements. However, when dealing with RDI, other indicators that account for plant water status must be used. Among these, several approaches have been studied such as trunk diameter fluctuations (TDF) (Fernández and Cuevas, 2010; Conejero et al., 2011), sap flow (Conejero et al., 2007) and stem water potential $\left(\Psi_{\mathrm{s}}\right)$ (Shackel et al., 2010). Sap flow and TDF have been reported less reliable than $\Psi_{\mathrm{s}}$ (Moriana et al., 2003; Intrigliolo and Castel, 2006). At commercial level, the use of leaf and stem water potential is considered more practical (Naor and Cohen, 2003; Bonet et al., 2010; Moriana et al., 2010).

Several studies proved the feasibility of using tentative threshold values of plant parameters as irrigation triggers (Besset et al., 2001; Goldhamer and Fereres, 2004). Girona et al. (2006) proved that leaf water potential thresholds could be successfully used for scheduling irrigation in vineyards and a similar approach has been suggested for peach orchards (Ghrab et al., 2013).

In this context, the aims of the current study were to (1) evaluate a methodology for scheduling peach orchard irrigation based on $\Psi_{\mathrm{s}}$ thresholds as a stress indicator for triggering irrigation, and (2) assess the effects of these RDI on peach tree physiology, vegetative growth, yield and fruit quality, as well as water savings, when compared to a fully irrigated control.

\subsection{Materials and Methods}

\section{Site description and plant material}

The experiment was conducted over three consecutive years (2008-2010) in a 0.5 ha plot of a commercial orchard located in Mula valley, Murcia, SE Spain $\left(37^{\circ} 55^{\prime} \mathrm{N}\right.$, $1^{\circ} 25^{\prime} \mathrm{W}, 360 \mathrm{~m}$ above sea level). The soil at the site is calcareous, stony, with a sandyloam texture, low organic matter content and its available water capacity is $0.25 \mathrm{~m}^{3} \mathrm{~m}^{-3}$, approximately. The climate is typically Mediterranean, with mild winters, hot dry summers and low rainfall. During the growing season (April-October), total rainfall was 254, 304 and $188 \mathrm{~mm}$ in 2008, 2009 and 2010, respectively; whereas reference crop evapotranspiration $\left(\mathrm{ET}_{0}\right)$ was 792, 817 and $784 \mathrm{~mm}$ in 2008, 2009 and 2010, respectively. 
Plant material consisted of peach trees [Prunus persica (L.) Batsch cv. 'Catherine'] planted in 1999 and grafted on GF-677 rootstock. Spacings were 4 m x 6 $\mathrm{m}$. Hand thinning was used to space fruitlets $20 \mathrm{~cm}$ along the fruit bearing shoots in order to achieve a commercial crop load. Pest control was that commonly used by the growers and all treatments received the same fertilization $\left(\mathrm{N}-\mathrm{P}_{2} \mathrm{O}_{5}-\mathrm{K}_{2} \mathrm{O}\right)$, applied through the drip irrigation system $\left(275-125-200 \mathrm{~kg} \mathrm{ha}^{-1}\right.$ year $\left.^{-1}\right)$ over the study period.

\section{Irrigation treatments and experimental design}

Irrigation water was supplied through a drip irrigation system, one pipeline for each row, with three emitters per tree (delivering $4 \mathrm{~L} \mathrm{~h}^{-1}$ each). The irrigation water was considered of good quality with a very low electrical conductivity $\left(0.6 \mathrm{dS} \mathrm{m}^{-1}\right)$.

Crop irrigation requirements were scheduled weekly according to daily $\mathrm{ET}_{0}$, calculated using the Penman-Monteith equation (Allen et al., 1998), and a local crop factor based on the time of the year and used in this region at commercial level. Monthly average values of $\mathrm{K}_{\mathrm{c}}$ employed for the control treatment from April to October were $0.73,0.83,0.94,0.96,0.39,0.39$ and 0.39 , respectively. A total of 192 trees were used in this study. The experiment was laid out in completely randomized blocks with 4 replications (16 trees each). The four central trees were used for measurements, and the other 12 trees acted as guard.

Three irrigation treatments were applied based on peach tree water status (Figure 7.1): Control plants (treatment $C$ ) were daily irrigated above the estimated crop evapotranspiration in order to obtain non-limiting soil water conditions (average 120\% $\mathrm{ET}_{\mathrm{c}}$ in three years of experiments), and two RDI strategies that consisted of a full irrigation during critical periods, and water restrictions during the non-critical periods of crop development (stage II of fruit growth and postharvest period). These RDI treatments were:

a) RDI I (Moderate deficit) plants were irrigated to maintain $\Psi_{\mathrm{s}}$ values close to 1.5 MPa during stage II of fruit growth and postharvest.

b) RDI II (Severe deficit) plants were irrigated to maintain $\Psi_{\mathrm{s}}$ values close to 1.8 $\mathrm{MPa}$ at stage II of fruit growth and $-2.0 \mathrm{MPa}$ at postharvest. 


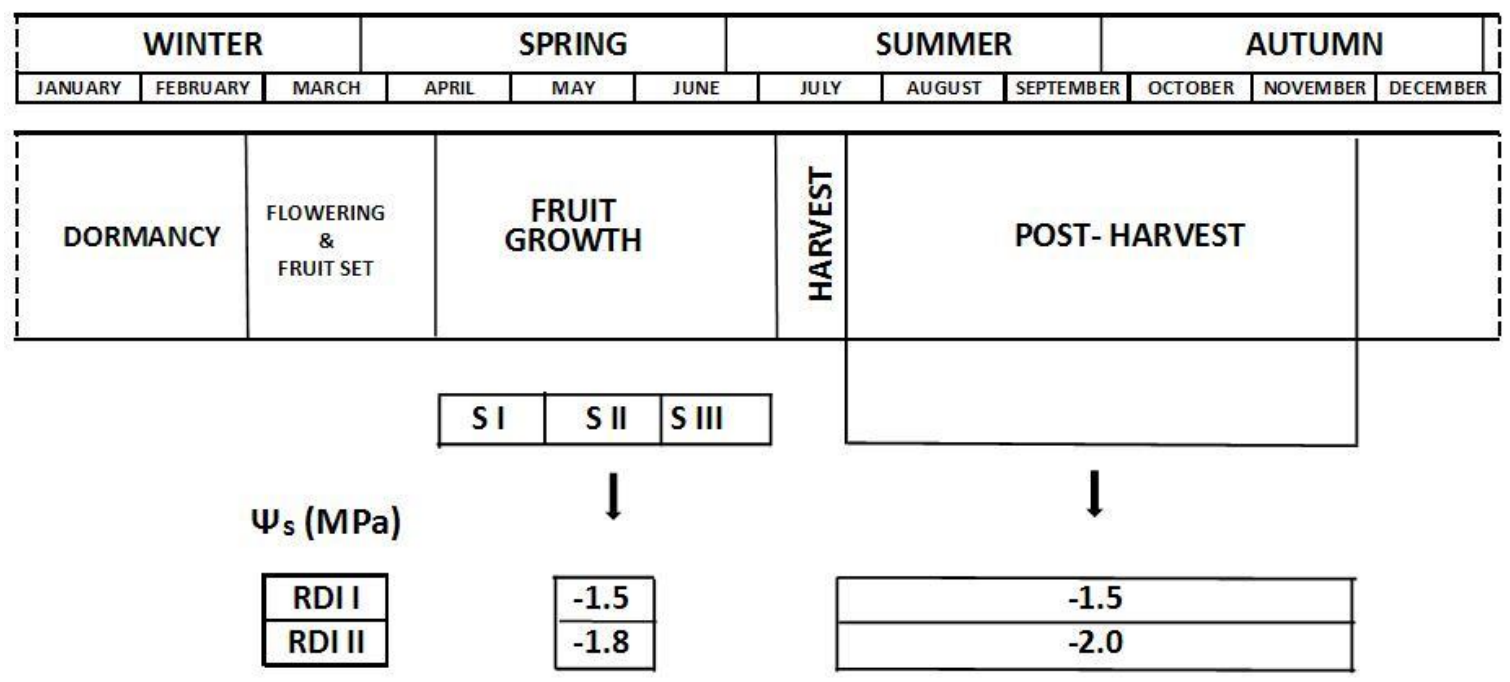

Figure 7.1. Regulated deficit irrigation (RDI I: moderate deficit, RDI II: severe deficit) strategies based on threshold values for midday stem water potential $\left(\Psi_{\mathrm{s}}\right)$, applied in stage II (S II) of fruit growth and postharvest during the three years of the experiment.

The irrigation rate was decreased by $10 \%$ when stem water potential on at least two out of three consecutive days was at or below the threshold value. The irrigation rate was increased by $10 \%$ when the stem water potential on at least two out of three consecutive days exceeded the threshold value. This irrigation protocol was based on that proposed by Goldhamer and Fereres (2001) for mature trees grown with high frequency irrigation.

Irrigation was automatically controlled by a head unit programmer and the amounts of water applied for each irrigation treatment were measured with in-line flowmeters placed in each experimental plot.

\section{Field and laboratory determinations}

\section{Climate data}

Climate data were recorded at an automatic weather station placed within the experimental orchard. Data on air temperature (maximum, minimum and average), solar radiation, air relative humidity, rainfall and wind speed $2.5 \mathrm{~m}$ above the soil surface, were collected every $15 \mathrm{~min}$. These data were used for calculating $\mathrm{ET}_{0}$ and crop water requirements.

\section{Soil water content}

The volumetric soil water content $\left(\theta_{\mathrm{v}}, \mathrm{m}^{3} \mathrm{~m}^{-3}\right)$ of the top $0.2 \mathrm{~m}$ of the soil profile was measured by time domain reflectometry (TDR) using a Tektronix device (Model 1502C, Tektronix Inc., OR), as described by Moreno et al. (1996). The $\theta_{\mathrm{v}}$ from $0.2 \mathrm{~m}$ 
down to a maximum depth of $1.0 \mathrm{~m}$ was measured every $0.1 \mathrm{~m}$ using a neutron probe (Model 4300, Troxler Electronic Laboratories Inc., NC), in access tubes installed $1.0 \mathrm{~m}$ from the trees and beside the emitters. The probes were placed at $0.2 \mathrm{~m}$ from the perperdicular emitter and next to the TDR rods. Measurements using one neutron probe and TDR per experimental plot (3 replications per treatment) were taken in the morning, every 7 to 15 days, during the experimental period.

\section{Stem water potential}

Midday stem water potential $\left(\Psi_{\mathrm{s}}\right)$ was measured using a pressure chamber (Soil Moisture Equip. Corp, model 3000, Santa Barbara, CA, USA) following the procedures described by Turner (1981).

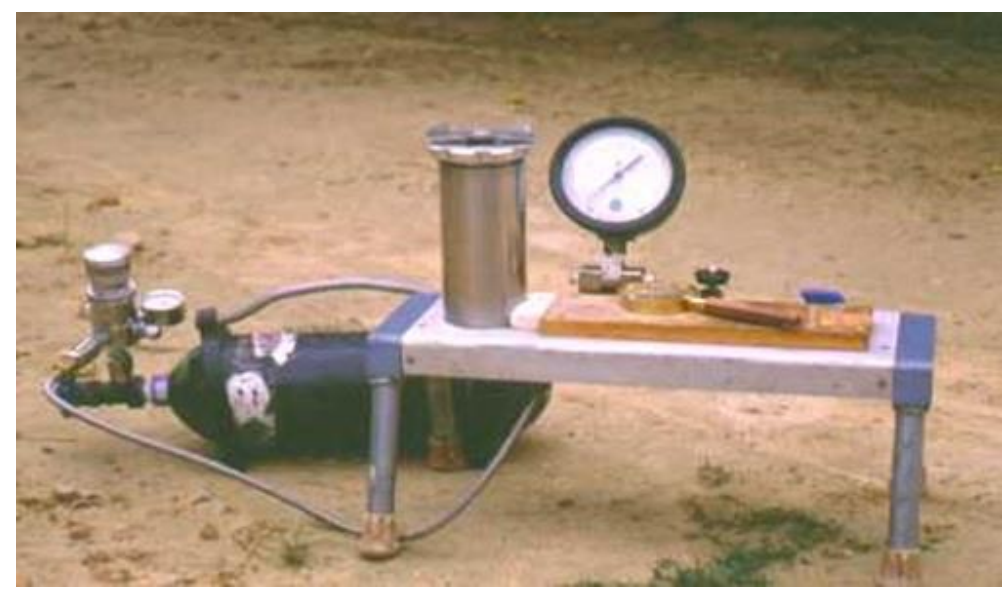

Picture 7.1. Pressure chamber (Own source).

Measurements were performed on mature leaves (one per tree) from the north face of the trunk, in the four central trees of each experimental plot. Leaves were enclosed in plastic bags covered with aluminum foil at least two hours prior to the measurements, which were carried out between 12:00 and 13:00 h solar time.

\section{Gas exchange parameters}

Net photosynthesis $\left(\mathrm{P}_{\mathrm{n}}\right)$ and stomatal conductance $\left(\mathrm{g}_{\mathrm{s}}\right)$ were measured at solar midday, in one fully expanded sun-facing leaf in the four central trees of each experimental plot per treatment, in the same days that stem water potential was recorded, using a field-portable photosynthesis system (LI-6400, Li-Cor, Lincoln, NE, USA). 


\section{Fruit growth and yield}

Fruit diameter was measured perpendicularly to the fruit suture on 160 fruits per treatment (40 fruits per replication) every 7-10 days. Each date, 10 fruits per tree were randomly selected in the four inner trees per experimental plot and their diameters measured using a digital caliper (Powerfix model Nr Z22855F, Milomex Ltd, Bedfordshire, UK).

Fruits from each tree were individually harvested on July in two or three commercial picks, depending on market demands. The number of fruits per tree was obtained by counting the fruits in the four inner trees (16 trees per treatment). The effect of the irrigation treatments on fruit size was studied evaluating marketable and nonmarketable production, as the proportion of fruits with a diameter above or below 56 $\mathrm{mm}$, respectively.

According to Commission Regulation (EC) $\mathrm{n}^{\mathrm{o}} 1861 / 2004,56 \mathrm{~mm}$ is the minimum diameter for a fruit to be considered in the "extra" category, which can be subdivided into different categories: AAAA (diameter > $90 \mathrm{~mm})$, AAA $(90 \mathrm{~mm}>$ diameter $\geq 80 \mathrm{~mm})$, AA $(80 \mathrm{~mm}>$ diameter $\geq 73 \mathrm{~mm})$, A $(73 \mathrm{~mm}>$ diameter $\geq 67 \mathrm{~mm})$, B $(67 \mathrm{~mm}>$ diameter $\geq 61 \mathrm{~mm}), \mathrm{C}(61 \mathrm{~mm}>$ diameter $\geq 56 \mathrm{~mm})$ and out of "extra" category D $(56 \mathrm{~mm}>$ diameter $\geq 51 \mathrm{~mm})$.

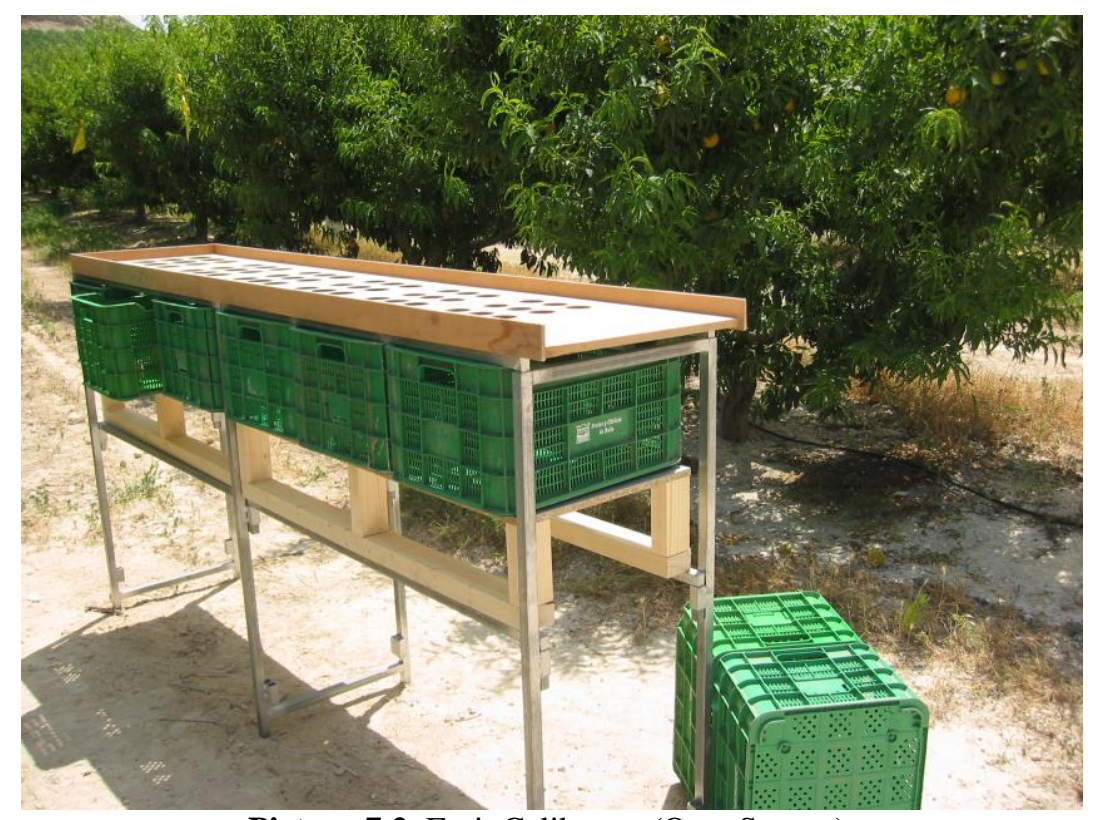

Picture 7.2. Fruit Calibrator (Own Source). 
Vegetative growth

Trunk diameter was measured annually during the dormancy stage in 64 trees per treatment with a sliding caliper, $0.20 \mathrm{~m}$ above the soil surface, and used to estimate trunk cross-sectional area (TCSA). Pruning weight was recorded for 16 trees per treatment (4 per replication) at winter pruning. Shoot length values were collected every 7-10 days by measuring the lengths of 10 randomly selected shoots in the four inner trees of each experimental plot per treatment.

\section{Fruit quality}

At harvest, 100 fruits per treatment (25 fruits per experimental plot) were randomly selected for quality assessment. Firmness, $\mathrm{pH}$, soluble solids content (SSC) and titratable acidity (TA) were evaluated as quality indices. Chemical analyses were conducted according to Artés et al. (1993). Fruit firmness was evaluated using a Durofel penetrometer DFT100 (Agro-Technologie S.A., Paris, France).

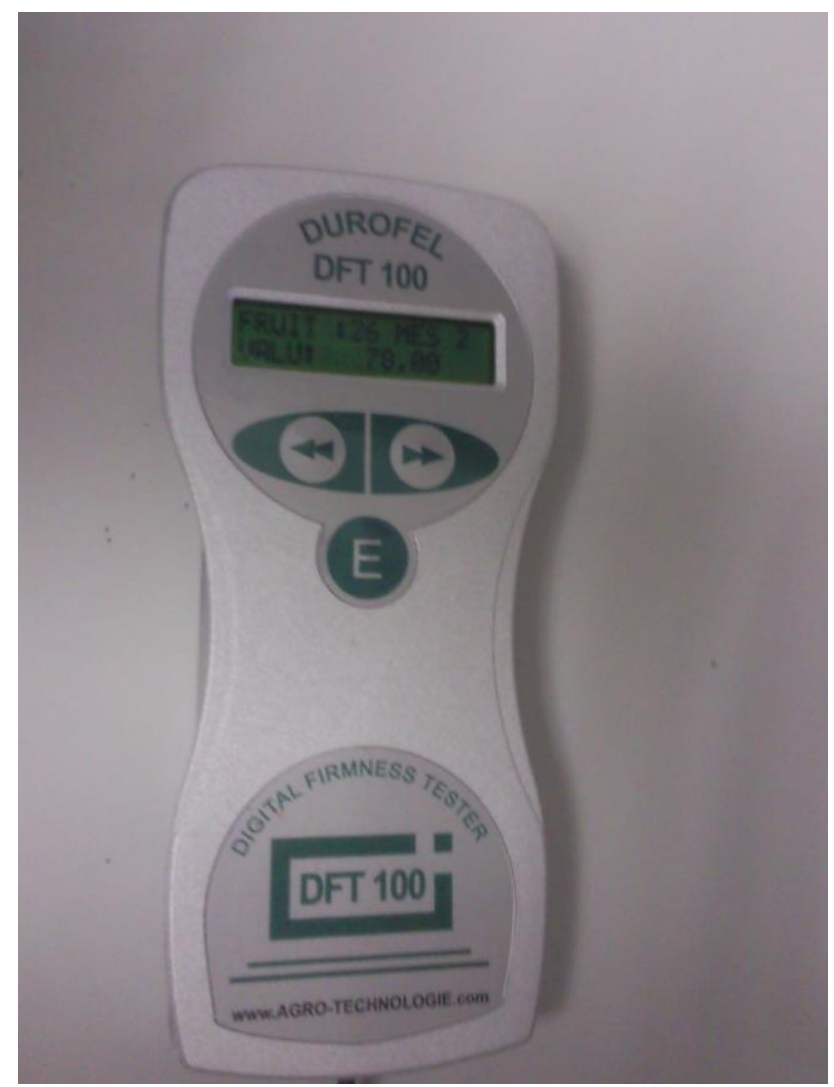

Picture 7.3. Durofel penetrometer (Own Source).

Juice was extracted from combined samples of longitudinal unpeeled slices. Total soluble solids concentration (SSC) was determined with a hand refractometer (Atago, Co., Japan); values were expressed as ${ }^{\circ}$ Brix. Titratable acidity (TA) was 
measured by titration of $5 \mathrm{~mL}$ of juice with $0.1 \mathrm{~mol} \mathrm{~L}^{-1} \mathrm{NaOH}$ to $\mathrm{pH} 8.1$ using an automatic titration system and expressed as $g$ malic acid $\mathrm{L}^{-1}$. The maturity index (MI), which affects the perception of taste (sweetness and acidity) was calculated as the ratio of SSC to TA. pH was measured using a pH-meter (Crison, Barcelona, Spain).

\section{Water stress integral}

The water stress integral (MPa-days) was calculated from the $\Psi_{\mathrm{s}}$ data in order to evaluate the intensity of water stress, according to the following equation (Myers, 1988):

$$
S_{\Psi}=\left|\sum_{i=0}^{i=t}\left(\bar{\Psi}_{i, i+1}-c\right) n\right|
$$

where:

$\Psi_{\mathrm{i}, \mathrm{i}+1}$ is the average $\Psi_{\mathrm{s}}$ for each time interval

$c$ is the value of the maximum $\Psi_{\mathrm{s}}$ in all seasons

$n$ is the number of days in the interval.

\section{Statistical analysis}

Statistical analysis was performed as a weighted analysis of variance (ANOVA; statistical software IBM SPSS Statistics v. 21 for Windows). The Shapiro-Wilk test was used to evaluate the normality of the data. Tukey's HSD test was used for mean separation. Percentage values of fruit categories were arcsine-transformed before statistical analysis. Unless otherwise stated, the significance level was $p \leq 0.05$.

\subsection{Results}

Seasonal evaporative demand was quite similar from year to year, ranging between $784 \mathrm{~mm}$ in 2010 and $817 \mathrm{~mm}$ in 2009. Rainfall was more variable during the experimental period, from $188 \mathrm{~mm}$ during the 2010 growing season to $304 \mathrm{~mm}$ in 2009 . Rainfall was relatively important during the vegetative phase and postharvest, although more than $50 \mathrm{~mm}$ were registered during stage II of fruit development in 2008 (Figure 7.2). During the experimental period, rainfall accounted for an average of $31 \%$ of total $\mathrm{ET}_{0}$ over the growing season. 


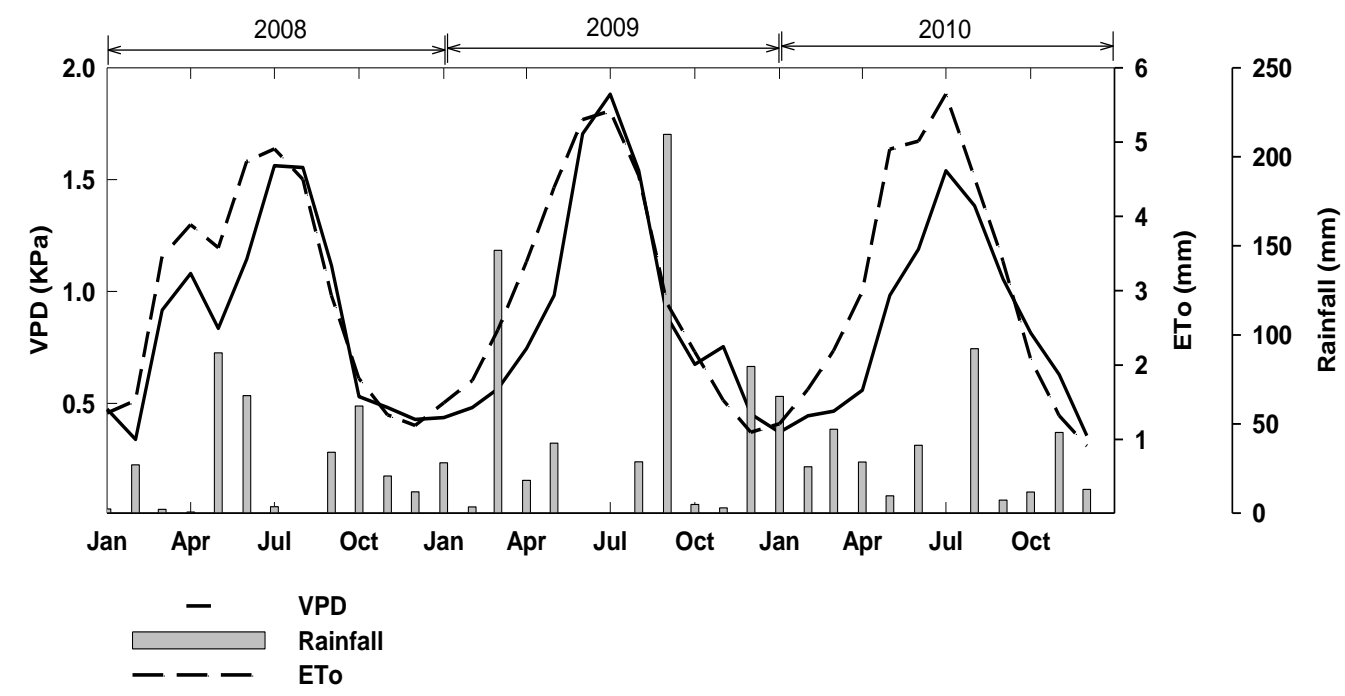

Figure 7.2. Reference evapotranspiration $\left(\mathrm{ET}_{0}\right)$, rainfall $(\mathrm{mm})$ and VPD $(\mathrm{KPa})$ in Fuente Librilla. Monthly values from data collected during 2008, 2009 and 2010.

Soil water content from 0 to $1 \mathrm{~m}$ depth (Figure 7.3), was nearly constant over the experimental period for $\mathrm{C}$ treatment, with values close to field capacity. In the RDI treatments, $\theta_{\mathrm{v}}$ decreased during the stage II of fruit development when compared to C, and recovered in stage III, when full irrigation was restored. During the postharvest period, the $\theta_{\mathrm{v}}$ decreased as a result of the deficit irrigation applied, reaching significantly lower values in RDI I ( $\left.\Psi_{\mathrm{s}}-1.5 \mathrm{MPa}\right)$ and RDI II ( $\left.\Psi_{\mathrm{s}}-2.0 \mathrm{MPa}\right)$ when compared to the $\mathrm{C}$ treatment (Figure 7.3).

Midday stem water potential $\left(\Psi_{\mathrm{s}}\right)$ used as a threshold for irrigation scheduling revealed clear differences in $\Psi_{\mathrm{s}}$ average values for each irrigation treatment (Figure 7.4B). In the early part of the season, $\Psi_{\mathrm{s}}$ below the thresholds for RDI I and RDI II trees were rarely observed; only by the end of stage II of fruit development in 2009. Consequently, little water was applied to the RDI trees during this stage (Figure 7.4A). However, RDI trees presented significantly lower $\Psi_{\mathrm{s}}$ values than $\mathrm{C}$ trees during stage II of fruit development. When irrigation was restored in stage III, $\Psi_{\mathrm{s}}$ was similar for the three treatments. 

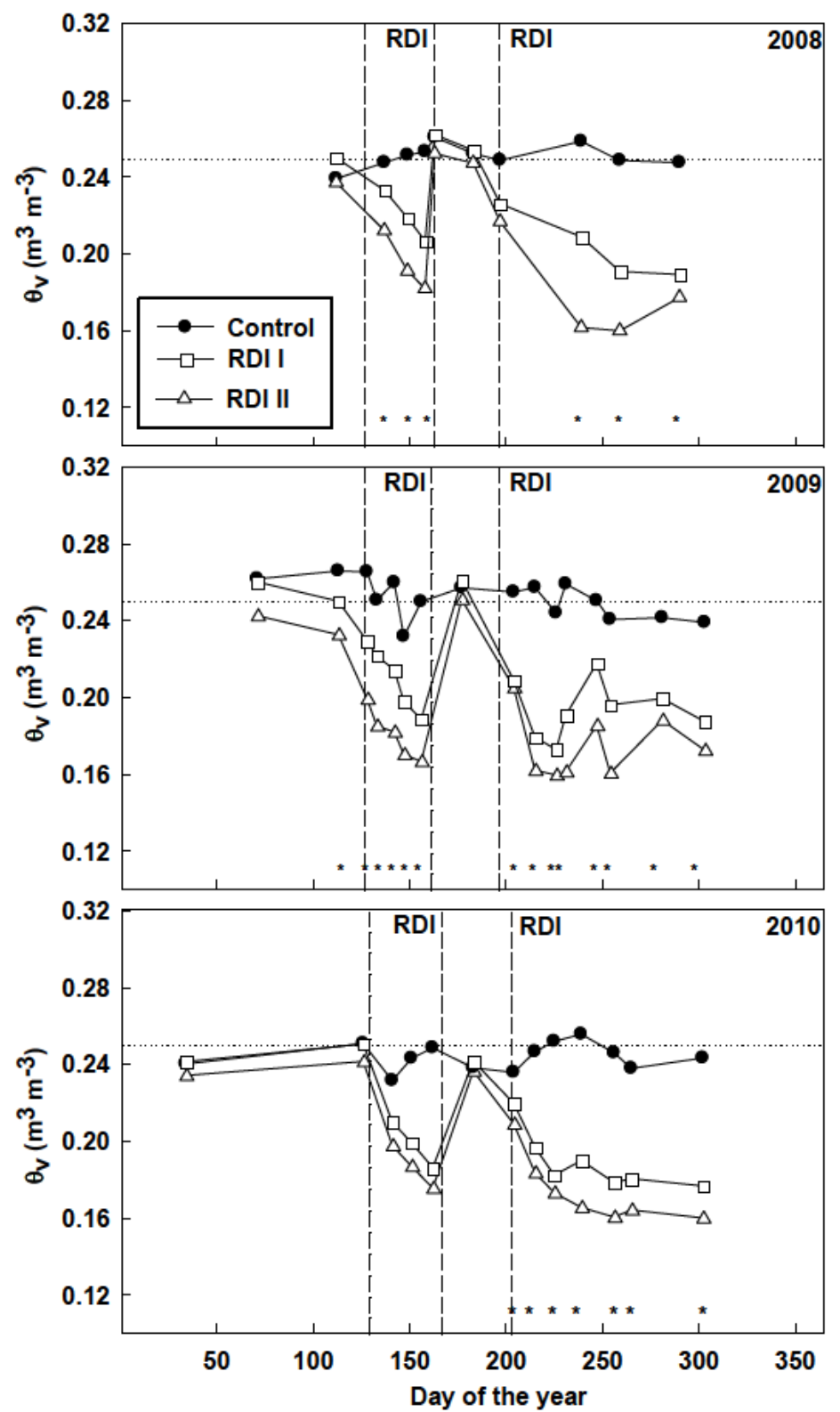

Figure 7.3. Volumetric soil water content down to $1 \mathrm{~m}$ depth $\left(\theta_{\mathrm{v}}\right)$, in $\mathrm{C}$ (closed circles), RDI I (open squares) and RDI II (open triangles) treatments for the three growing seasons studied (2008-2010). Each data point is the mean of three values. Asterisks indicate significant differences among treatments $(p<0.05)$. The interval between vertical lines, from left to right, represents the beginning of stages II and III of fruit growth and postharvest. Horizontal lines represent field capacity. 


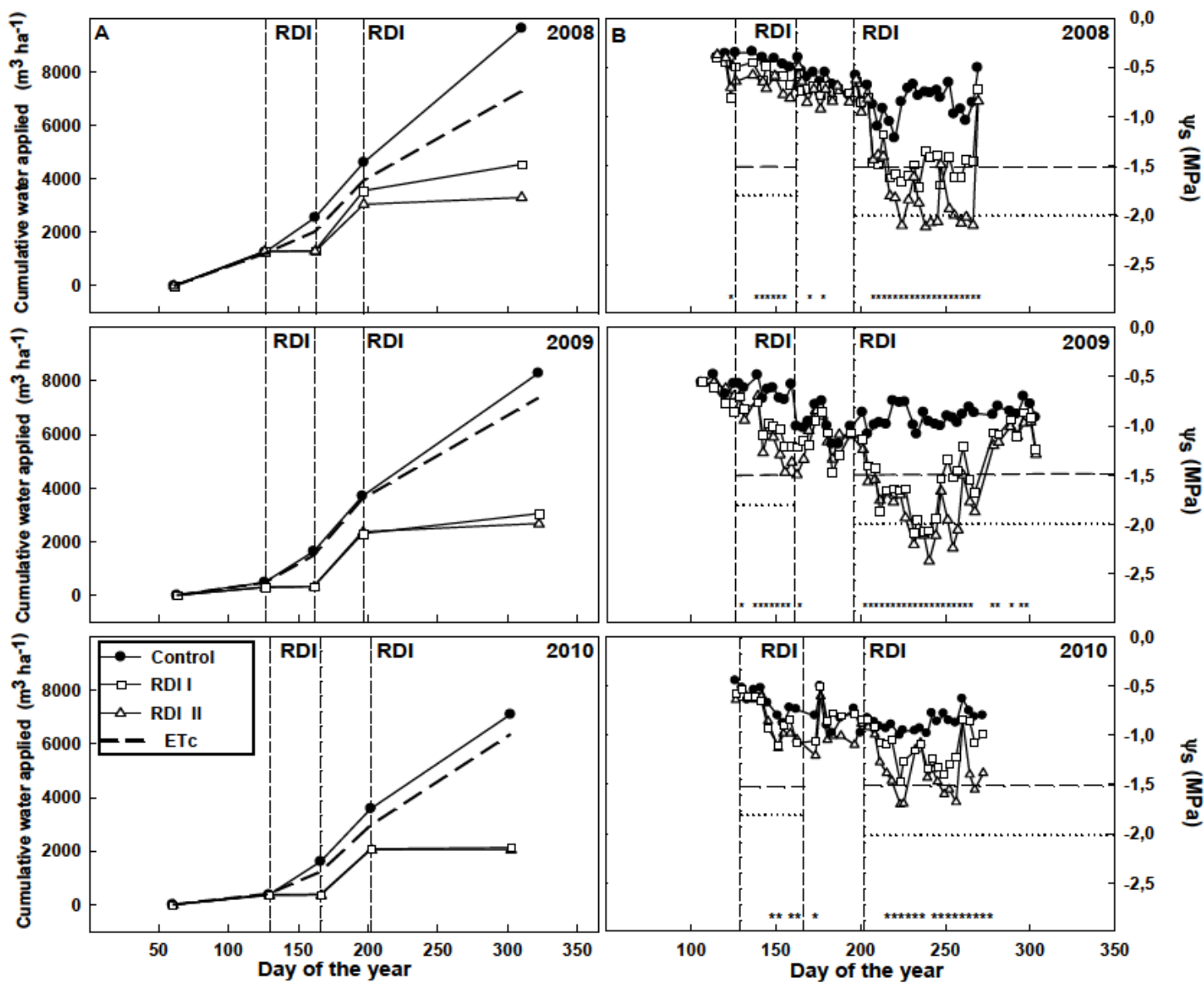

Figure 7.4. Seasonal patterns of (A) cumulative applied water and (B) midday stem water potential values for each irrigation treatment for the 3 years of the experiment: C (closed circles), RDI I (open squares) and RDI II (open triangles) plants. Each data point is the mean of sixteen values. Asterisks indicate significant differences among treatments $(p<0.05)$. The interval between vertical lines, from left to right, represents the beginning of stages II and III of fruit growth and postharvest. Horizontal lines represent threshold values in each phenological stage for both RDI treatments.

During postharvest, $\Psi_{\mathrm{s}}$ was below the thresholds most of the time in 2008 and 2009 (Figure 7.4B), with a resulting increase in irrigation rate during this period (Figure 7.4A). Differences in $\Psi_{s}$ between both RDI treatments were significant on certain dates; however, the lower threshold for irrigation triggering in RDI II (-2.0 MPa) was rarely observed in 2009 and never reached in 2010 due to the specific climate conditions of those years and, consequently, lower irrigation water amounts were applied to this treatment (Figure 7.4B).

Average midday stem water potential values for each stage in the growing cycle showed clear differences between treatments, mainly at postharvest, and these were repeated with the same trends for the three years of the experiment (Table 7.1). During the stage I and stage III of fruit development, $\Psi_{\mathrm{s}}$ values were very similar among 
treatments, except in stage III of fruit development in 2008. The decrease of $\Psi_{\mathrm{s}}$ in RDI treatments compared to control was about 40-51\% during 2008, 39-46\% during 2009 and 6-26\% during 2010 for the RDI I and RDI II treatments, respectively.

Table 7.1. Average midday stem water potential in different stages of fruit growth for the three studied growing seasons. Each data is the mean of all values in the corresponding stage. Different letters next to a value in each year indicate significant differences among treatments according to Tukey's multiple range test $(p<0.05)$.

\begin{tabular}{ccccc}
\hline & Stage I & Stage II & Stage III & Postharvest \\
\hline 2008 & & & & \\
C & $-0.40 a$ & $-0.43 a$ & $-0.60 a$ & $-0.85 a$ \\
RDI I & $-0.53 a$ & $-0.57 b$ & $-0.71 a b$ & $-1.40 b$ \\
RDI II & $-0.53 a$ & $-0.68 b$ & $-0.74 b$ & $-1.69 c$ \\
2009 & & & & \\
C & $-0.57 a$ & $-0.62 a$ & $-0.99 a$ & $-0.90 a$ \\
RDI I & $-0.64 a$ & $-0.96 b$ & $-1.14 a$ & $-1.48 b$ \\
RDI II & $-0.58 a$ & $-1.05 b$ & $-1.14 a$ & $-1.66 b$ \\
2010 & & & & \\
C & $-0.45 a$ & $-0.67 a$ & $-0.82 a$ & $-0.86 a$ \\
RDI I & $-0.58 a$ & $-0.80 a$ & $-0.80 a$ & $-1.13 b$ \\
RDI II & $-0.64 a$ & $-0.82 a$ & $-0.97 a$ & $-1.36 c$ \\
\hline
\end{tabular}

The amounts of water applied during the first year of the experiment (2008) were 962, 454 and $330 \mathrm{~mm}$ for C, RDI I and RDI II, respectively (Figure 7.4A). These amounts were reduced in 2009 and 2010, and were similar for the two RDI treatments, namely 849, 289 and 247 mm for C, RDI I and RDI II, respectively, in 2009; and 710, 213 and $206 \mathrm{~mm}$ for C, RDI I and RDI II, respectively, in 2010. These differences were due to climate conditions that did not cause $\Psi_{\mathrm{s}}$ values to be lower than the scheduled thresholds in both RDI periods (stage II of fruit development and postharvest), thus irrigation was restricted to the point that hardly was applied in 2010 during porhasvest (Figure 7.4A).

As a consequence, when compared to $\mathrm{ET}_{\mathrm{c}}$, water reductions of $26-45 \%$ in 2008 , 49-55\% in 2009 and 64-65\% were achieved for RDI I and RDI II treatments, respectively. 


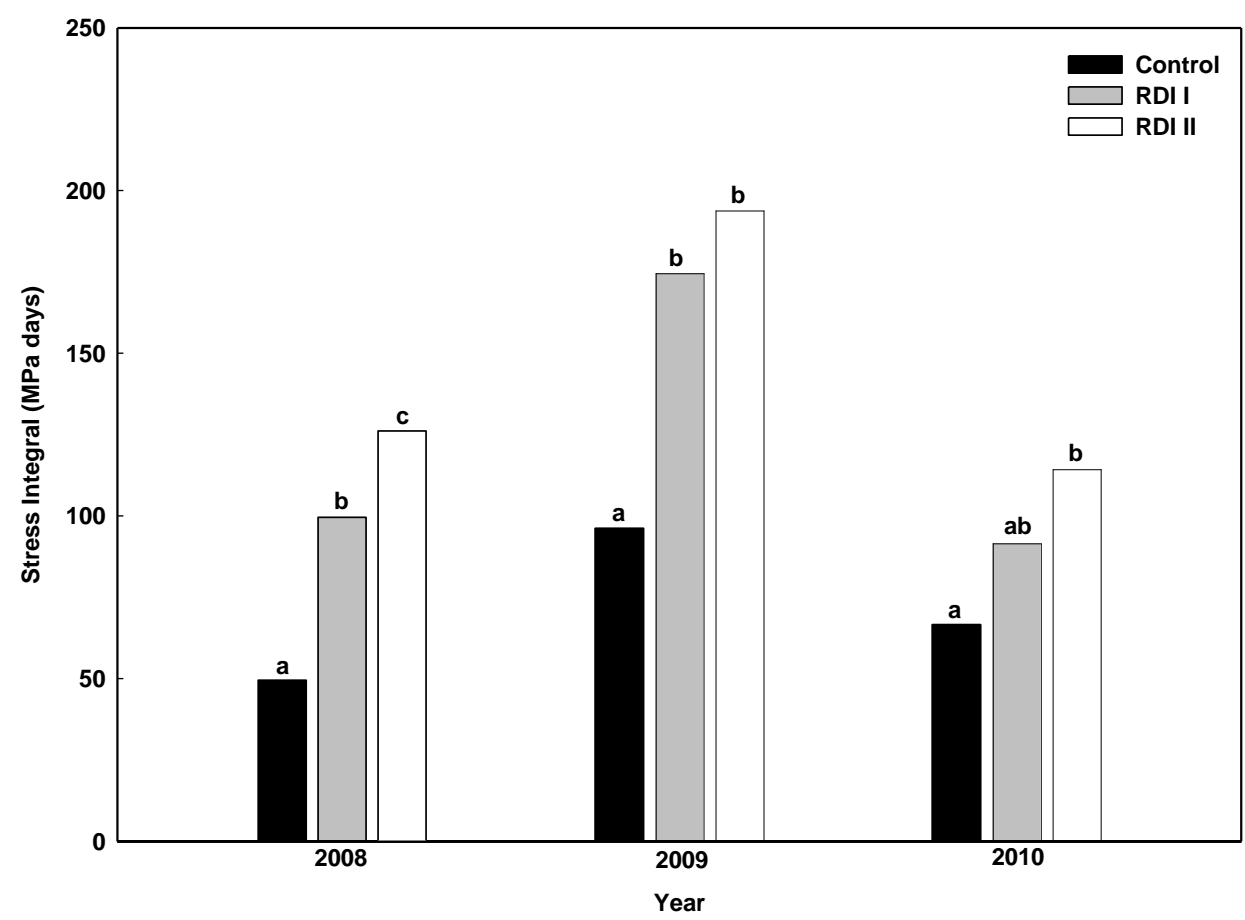

Figure 7.5. Stress integral for each irrigation treatment, control (black bars), RDI I (gray bars) and RDI II (white bars) in the 2008, 2009 and 2010 growing cycles. Different letters on top of bars indicate significant differences according to Tukey's multiple range test $(p<0.05)$.

Overall for the whole season, $\mathrm{S}_{\Psi}$ values reflected those significant differences among treatments, RDI II showed the highest values of water stress integral (Figure 7.5), but only significantly higher than RDI I in 2008. For stages of fruit development, water stress integral values reflected the differences in irrigation treatments, as observed by $\Psi_{\mathrm{s}}$.

During stage II of fruit development, sigficantly greater $S_{\Psi}$ values were detected for RDI I and RDI II treatments when compared to C (Table 7.2). However, these differences were not maintained in 2010, when a low evaporative demand made the values of $S_{\Psi}$ to be similar among treatments. However, at postharvest, RDI II reflected the highest $S_{\Psi}$ values, showing significant differences from RDI I only in 2008 (Table $7.2)$. 
Table 7.2. Stress integral average values (MPa days) in different stages during the three seasons of the experiment. These values were calculated with data of the midday stem water potential of Figure 7.4B. Each data point is the mean of all values in the corresponding stage. Different letters next to a value in each year indicate significant differences according to

Tukey's multiple range test $(p<0.05)$.

\begin{tabular}{ccccc}
\hline & Stage I & Stage II & Stage III & Postharvest \\
\hline 2008 & & & & \\
C & $0.65 a$ & $2.74 a$ & $10.69 a$ & $36.42 a$ \\
RDI I & $2.16 a$ & $8.18 b$ & $14.96 a$ & $76.76 b$ \\
RDI II & $1.83 a$ & $12.07 \mathrm{c}$ & $16.48 \mathrm{a}$ & $98.46 \mathrm{c}$ \\
2009 & & & & \\
C & $4.53 \mathrm{a}$ & $11.05 \mathrm{a}$ & $22.58 \mathrm{a}$ & $58.91 \mathrm{a}$ \\
RDI I & $6.84 \mathrm{a}$ & $23.65 \mathrm{~b}$ & $28.01 \mathrm{a}$ & $118.19 \mathrm{~b}$ \\
RDI II & $5.13 \mathrm{a}$ & $28.12 \mathrm{~b}$ & $27.53 \mathrm{a}$ & $135.84 \mathrm{~b}$ \\
2010 & & & & \\
C & $0.42 \mathrm{a}$ & $12.59 \mathrm{a}$ & $16.63 \mathrm{a}$ & $36.86 \mathrm{a}$ \\
RDI I & $0.64 \mathrm{a}$ & $18.28 \mathrm{a}$ & $19.82 \mathrm{a}$ & $54.96 \mathrm{~b}$ \\
RDI II & $0.73 \mathrm{a}$ & $19.03 \mathrm{a}$ & $25.92 \mathrm{a}$ & $70.99 \mathrm{~b}$ \\
\hline
\end{tabular}

Gas exchange parameters showed a similar trend over the growing season as those of $\Psi_{\mathrm{s}}$. Significant reductions in net photosynthesis rate $\left(\mathrm{P}_{\mathrm{n}}\right.$, Figure $\left.7.6 \mathrm{~A}\right)$ and leaf stomatal conductance $\left(\mathrm{g}_{\mathrm{s}}\right.$, Figure 7.6B) for the RDI treatments compared to $\mathrm{C}$ were observed at postharvest and in one date during the stage II of fruit development. The $\mathrm{P}_{\mathrm{n}}$ and $g_{s}$ values for the RDI treatments were, approximately, one third of those measured in $\mathrm{C}$ trees.

Pruning weight was significantly higher in the $C$ trees than in those subjected to RDI (Figure 7.7). A linear correlation between pruning weight and the amount of water applied was detected (Figure 7.8), with a significant determination coefficient $\left(\mathrm{r}^{2}=\right.$ $0.81, p<0.01)$.

In addition, TCSA was similar for the three treatments considered on the first year of the experiment (Table 7.3); however, this parameter was significantly lower for RDI trees in 2009 and 2010. 


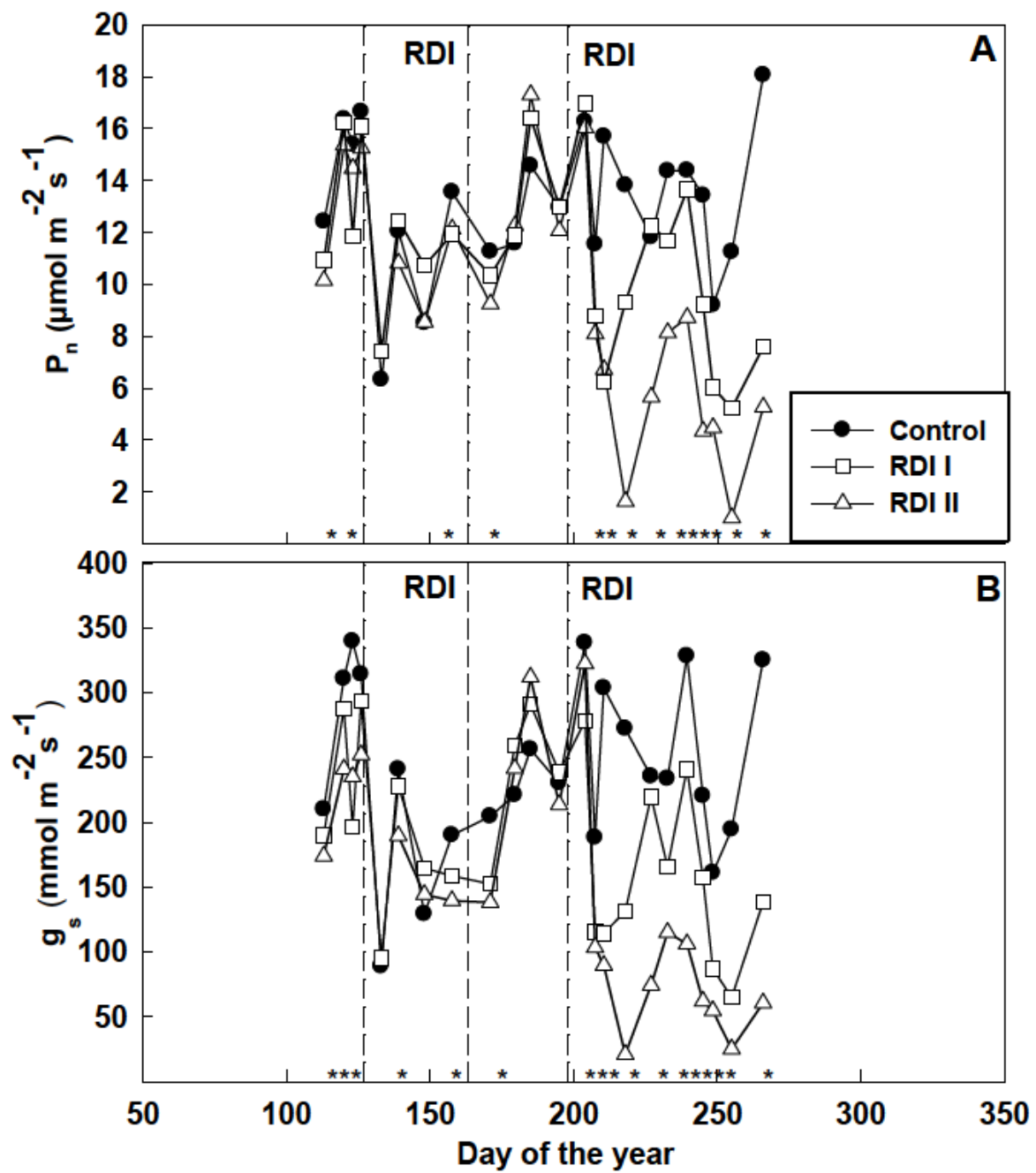

Figure 7.6. Net photosynthesis $\left(\mathrm{P}_{\mathrm{n}}, \mathrm{A}\right)$ and stomatal conductance $\left(\mathrm{g}_{\mathrm{s}}, \mathrm{B}\right)$ values averaged for the three studied growing seasons in C (closed circles), RDI I (open squares) and RDI II (open triangles) plants. Each data point is the mean of sixteen values. Asterisks indicate significant differences among treatments $(p<0.05)$. The interval between vertical lines, from left to right, represents the beginning of stages II and III of fruit growth and postharvest.

Vegetative growth was restricted by RDI when compared to C: Shoot length from RDI trees was significantly lower than that observed for $\mathrm{C}$ trees (Figure 7.9A), although no differences were observed between both RDI treatments by the end of the season. 


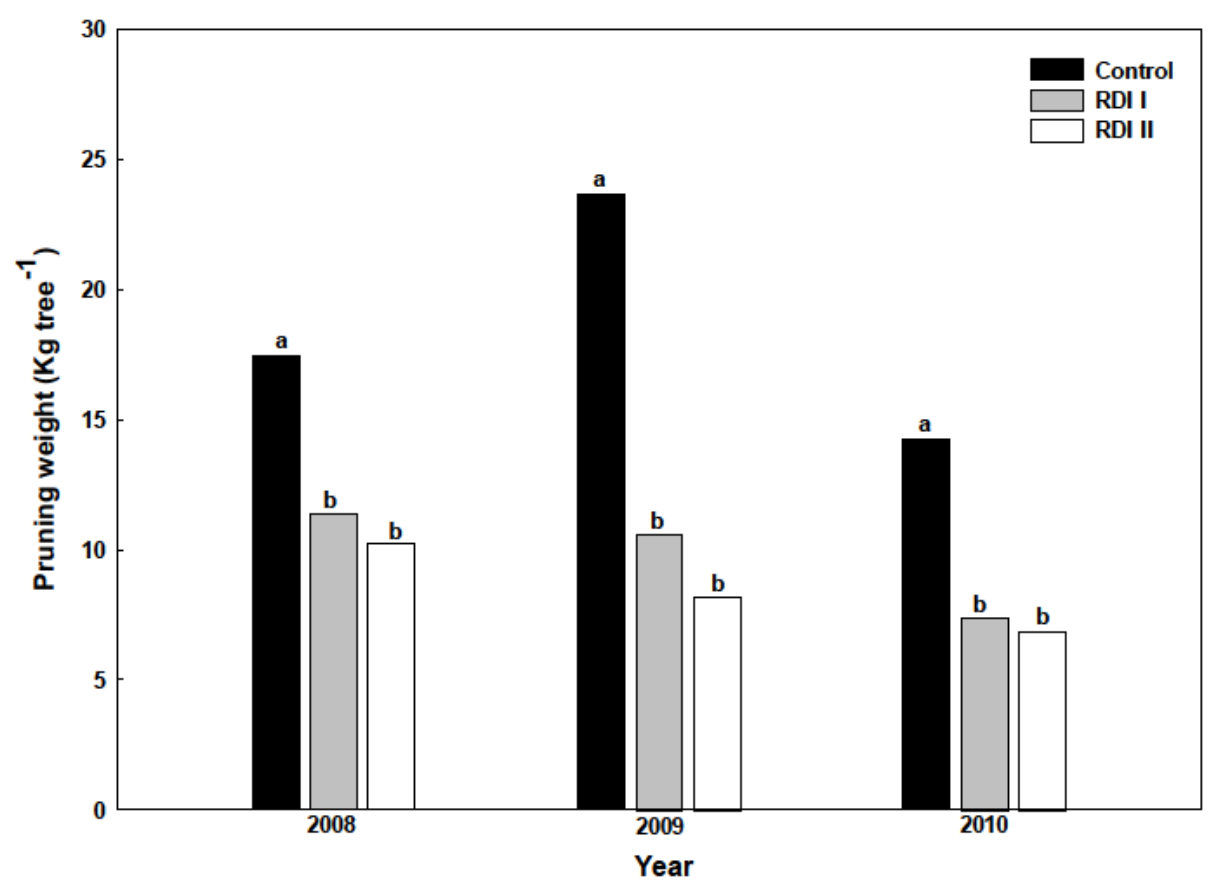

Figure 7.7. Pruning weight as a function of the irrigation treatment, control (black bars), RDI I (gray bars) and RDI II (white bars) in the 2008, 2009 and 2010 growing cycles. Each bar corresponds to the mean of sixteen trees. Different letters on top of bars indicate significant differences according to Tukey's multiple range test $(p<0.05)$.

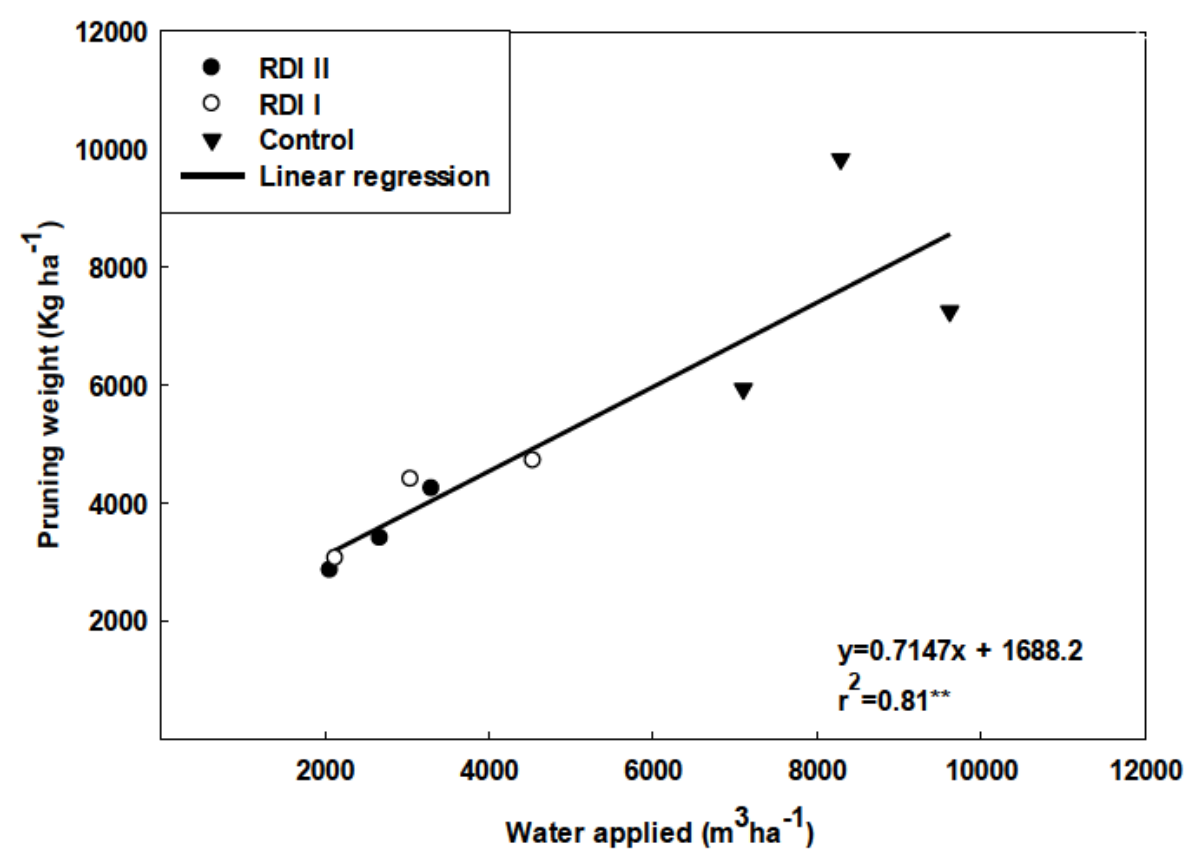

Figure 7.8. Relation between pruning weight and water applied in C (closed triangles), RDI I (open circles) and RDI II (closed circles) plants for the three studied growing seasons. Solid line represents the linear regression between pruning weight and water applied. Asterisks indicate a significant relationship between variables $(p<0.01)$ 
Table 7.3. Flower density, fruit set and trunk cross sectional area (TCSA) average values in different treatments during the three seasons of the experiment. Each data point is the mean of 160 values per treatment (Fruit set and flower density) and 64 values per treatment (TCSA). Different letters next to a value in each year indicate significant differences according to Tukey's multiple

\begin{tabular}{cccc}
\multicolumn{4}{c}{ range test $(p<0.05)}$. \\
\hline & $\begin{array}{c}\text { Flower density } \\
\left(\text { Fl cm }^{-1}\right)\end{array}$ & $\begin{array}{c}\text { Fruit set } \\
(\%)\end{array}$ & $\begin{array}{c}\text { TCSA } \\
\left(\mathrm{cm}^{2}\right)\end{array}$ \\
\hline 2008 & & & \\
C & $0.32 \mathrm{a}$ & $60.34 \mathrm{a}$ & $151.51 \mathrm{a}$ \\
RDI I & $0.32 \mathrm{a}$ & $58.73 \mathrm{a}$ & $149.78 \mathrm{a}$ \\
RDI II & $0.21 \mathrm{a}$ & $66.67 \mathrm{a}$ & $146.99 \mathrm{a}$ \\
2009 & & & \\
C & $0.48 \mathrm{~b}$ & $78.38 \mathrm{a}$ & $180.66 \mathrm{a}$ \\
RDI I & $0.60 \mathrm{ab}$ & $79.74 \mathrm{a}$ & $175.06 \mathrm{ab}$ \\
RDI II & $0.67 \mathrm{a}$ & $85.36 \mathrm{a}$ & $162.65 \mathrm{~b}$ \\
2010 & & & \\
C & $0.22 \mathrm{~b}$ & $87.01 \mathrm{a}$ & $208.44 \mathrm{a}$ \\
RDI I & $0.38 \mathrm{a}$ & $91.30 \mathrm{a}$ & $188.14 \mathrm{~b}$ \\
RDI II & $0.34 \mathrm{ab}$ & $94.82 \mathrm{a}$ & $186.54 \mathrm{~b}$ \\
\hline
\end{tabular}

On the contrary, fruit development was mainly unaffected by water restrictions. Fruit growth, expressed as fruit diameter, was very similar among treatments (Figure 7.9B), as well as fruit set (Table 7.3).

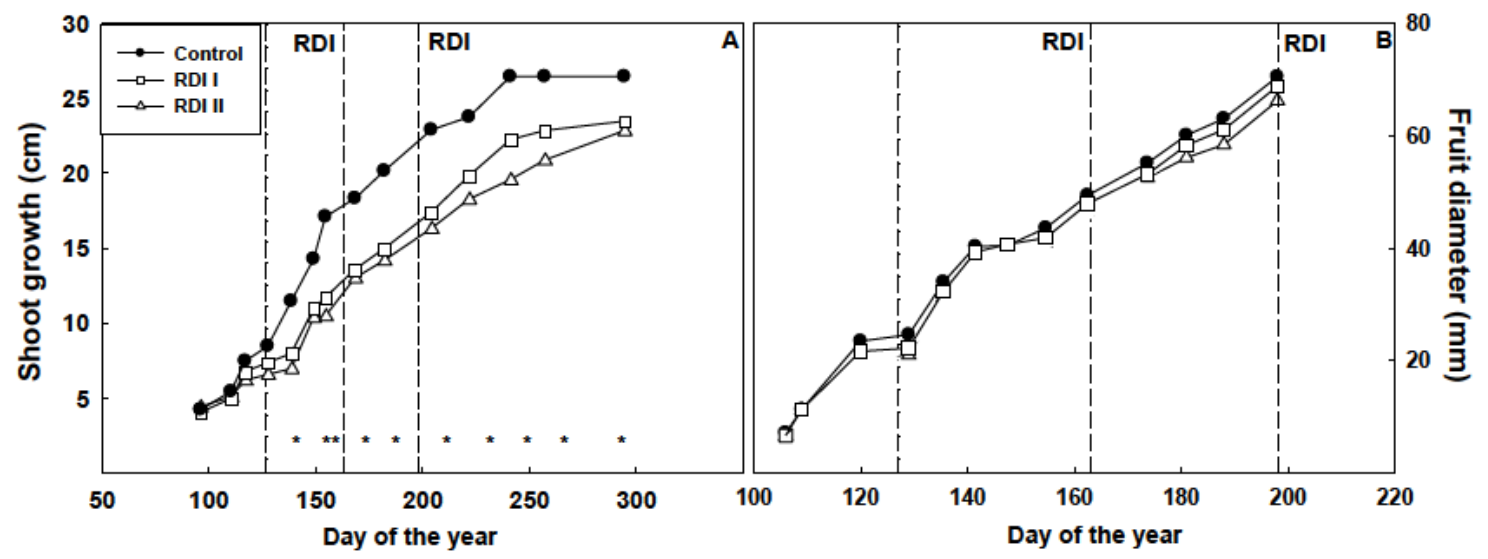

Figure 7.9. Shoot growth $(\mathrm{A}, \mathrm{cm})$ and fruit diameter $(\mathrm{B}, \mathrm{mm})$ evolution in $\mathrm{C}$ (closed circles), RDI I (open squares) and RDI II (open triangles) tress for the three studied growing seasons. The interval between vertical lines, from left to right, represents the beginning of stages II and III of fruit growth and postharvest. Each value is the mean of 160 measurements. 
Table 7.4. Crop load (number of fruits tree $\left.{ }^{-1}\right)$, yield $\left(\mathrm{kg}\right.$ tree $\left.^{-1}\right)$, water productivity (WP, $\mathrm{kg} \mathrm{m}^{-3}$ ) and water spends/savings in each non-critical period and total in response to the irrigation treatments for the experimental period 2008-2010. Different letters next to a value in each year indicate significant differences according to Tukey's multiple range test $(p<0.05)$.

\begin{tabular}{|c|c|c|c|c|c|c|}
\hline & $\begin{array}{c}\text { Crop load } \\
\text { (Fruits tree }^{-1} \text { ) } \\
\end{array}$ & $\begin{array}{c}\text { Yield } \\
\left(\text { Kg tree }^{-1}\right) \\
\end{array}$ & $\begin{array}{c}\mathrm{WP} \\
\left(\mathrm{Kg} \mathrm{m}^{-3}\right) \\
\end{array}$ & SII (\%) & $\begin{array}{r}\text { Water savings } \\
\text { Postharvest (\%) }\end{array}$ & Total $(\%)$ \\
\hline \multicolumn{7}{|l|}{2008} \\
\hline C & 170a & 29.87a & $1.29 a$ & -58 & -50 & -32 \\
\hline RDI I & $137 a$ & 26.99a & $2.47 \mathrm{ab}$ & 97 & 74 & 38 \\
\hline \multicolumn{6}{|l|}{2009} & 55 \\
\hline $\mathbf{C}$ & 489a & $67.23 \mathrm{a}$ & $3.30 \mathrm{a}$ & -13 & -22 & -12 \\
\hline RDI I & $523 a$ & $66.54 a$ & $9.73 b$ & 98 & 80 & 59 \\
\hline \multicolumn{6}{|l|}{2010} & 64 \\
\hline C & 173a & $30.16 a$ & $1.77 \mathrm{a}$ & -50 & -4 & -12 \\
\hline RDI I & $197 a$ & 33.79a & $6.61 b$ & 99 & 98 & 67 \\
\hline RDI II & $244 a$ & 40.40a & 8.15b & 99 & 99 & 68 \\
\hline
\end{tabular}

Flower density followed an opposite trend as that of TCSA, namely, it was unaffected in 2008 but was higher in RDI trees when compared to C trees in 2009 and 2010 (Table 7.3). Crop load and yield were similar among treatments although highly variable between years (Table 7.4). Furthermore, no differences among treatments were observed for fruit distribution into commercial categories (Figure 7.10).

The fact that similar yields were obtained for the three treatments studied made that water productivity (WP) values were higher under RDI treatments when compared to control (Table 7.4). Therefore, important water savings were registered for RDI treatments, being greater under RDI II than for RDI I (Table 7.4). When compared to control and depending on the year, RDI I supposed between 38 and $67 \%$ water savings for the whole season, whereas RDI II saved between 55 and $68 \%$ than C.

Fruit quality parameters reflected certain differences among treatments (Table 7.5). Fruit firmness was unaffected by irrigation treaments and titratable acidity was only affected in 2010 , although $\mathrm{pH}$ showed a tendency to reach lower values under RDI treatments. On the contrary, SSC was significantly higher under RDI when compared to C. This caused that maturity index (MI) was greater in RDI than in C, except for 2009 (Table 7.5). 

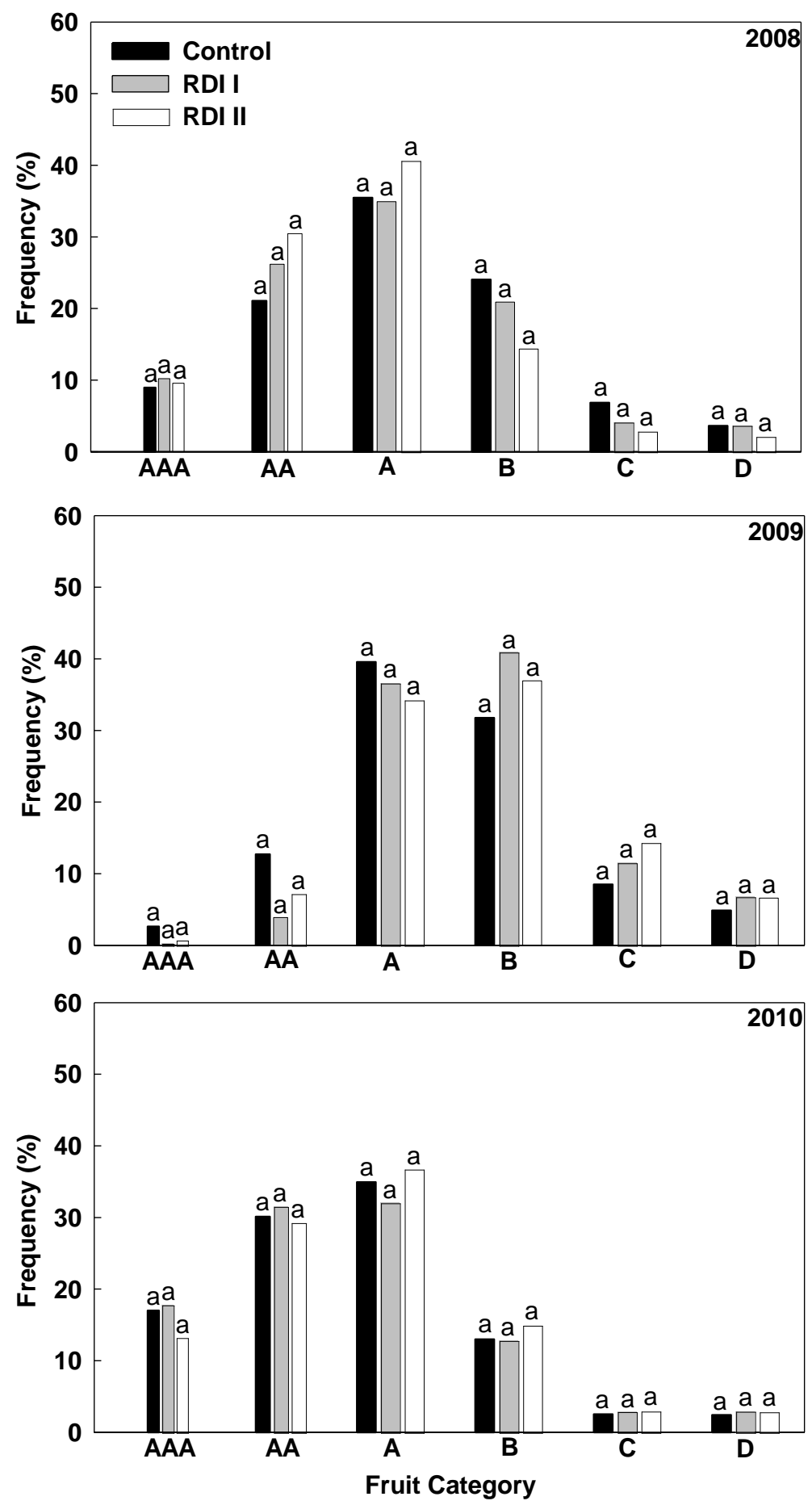

Figure 7.10. Percentage of fruit categories for each irrigation treatment, control (black bars), RDI I (gray bars) and RDI II (white bars) in the 2008, 2009 and 2010 growing cycles. Each bar corresponds to the mean of sixteen trees. Different letters on top of bars indicate significant differences according to Tukey's multiple range test $(p<0.05)$. 
Table 7.5. Fruit firmness (N), pH, soluble solids content ( $\left.\mathrm{SSC},{ }^{\circ} \mathrm{Brix}\right)$, tritratable acidity (TA, g $100 \mathrm{~mL}^{-1}$ ) and SSC/TA ratio at harvest for all treatments. Values are the mean of 100 measurements. Different letters next to a value in each year indicate significant differences according to Tukey's multiple range test $(p<0.05)$.

\begin{tabular}{cccccc}
\hline & $\begin{array}{c}\text { Firmness } \\
(\mathrm{N})\end{array}$ & $\mathrm{pH}$ & $\begin{array}{c}\text { SSC } \\
\left({ }^{\circ} \mathrm{Brix}\right)\end{array}$ & $\begin{array}{c}\text { TA } \\
\left(\mathrm{g} 100 \mathrm{~mL}^{-1}\right)\end{array}$ & $\begin{array}{c}\text { Maturity Index } \\
(\text { SSC/TA })\end{array}$ \\
\hline 2008 & & & & & \\
C & $50.47 \mathrm{a}$ & $4.13 \mathrm{a}$ & $10.64 \mathrm{a}$ & $0.59 \mathrm{a}$ & $18.82 \mathrm{a}$ \\
RDI I & $48.80 \mathrm{a}$ & $3.98 \mathrm{~b}$ & $11.16 \mathrm{a}$ & $0.52 \mathrm{a}$ & $21.93 \mathrm{~b}$ \\
RDI II & $51.74 \mathrm{a}$ & $3.92 \mathrm{~b}$ & $11.23 \mathrm{a}$ & $0.54 \mathrm{a}$ & $21.78 \mathrm{~b}$ \\
2009 & & & & & \\
C & $49.29 \mathrm{a}$ & $3.86 \mathrm{a}$ & $10.61 \mathrm{ab}$ & $0.61 \mathrm{a}$ & $17.91 \mathrm{a}$ \\
RDI I & $49.10 \mathrm{a}$ & $3.93 \mathrm{~b}$ & $10.40 \mathrm{a}$ & $0.57 \mathrm{a}$ & $18.82 \mathrm{a}$ \\
RDI II & $51.45 \mathrm{a}$ & $3.82 \mathrm{a}$ & $11.36 \mathrm{~b}$ & $0.61 \mathrm{a}$ & $19.23 \mathrm{a}$ \\
2010 & & & & & \\
C & $52.23 \mathrm{a}$ & $3.88 \mathrm{a}$ & $11.51 \mathrm{a}$ & $0.75 \mathrm{a}$ & $15.60 \mathrm{a}$ \\
RDI I & $56.45 \mathrm{a}$ & $3.97 \mathrm{a}$ & $12.09 \mathrm{ab}$ & $0.64 \mathrm{~b}$ & $19.23 \mathrm{~b}$ \\
RDI II & $56.45 \mathrm{a}$ & $3.88 \mathrm{a}$ & $13.07 \mathrm{~b}$ & $0.72 \mathrm{ab}$ & $18.23 \mathrm{~b}$ \\
\hline
\end{tabular}

\subsection{Discussion}

During pit hardening (Stage II), peach fruit growth is not very sensitive to water stress (Chalmers et al., 1981). In this study, the effects of two different RDI treatments on peach fruit growth were negligible during the three years of the experiment. These RDI strategies were applied at stage II of fruit development and at postharvest based on $\Psi_{\mathrm{s}}$ thresholds, as proposed by Girona et al. (2006), since this indicator of plant water status is very sensitive to water deprivation (Remorini and Massai, 2003).

The use of $\Psi_{\mathrm{s}}$ for irrigation scheduling has progressed in recent years, especially for managing RDI in fruit trees and vines (Girona et al., 2006; Shackel et al., 2010). This indicator provides more site-specific information than the water balance method and, therefore, it can account for spatial variations (Girona et al., 2006).

A wide range of tree water status was observed as a result of the different watering conditions imposed. At the beginning of the season, $\Psi_{\mathrm{s}}$ was very similar for the three treatments considered, around -0.5 MPa. During stage II of fruit development, significant differences were observed between fully irrigated trees and those subjected to RDI due to water withholding. However, values close to the predetermined $\Psi_{\mathrm{s}}$ thresholds were rarely observed during this stage and, consequently, low water amounts were applied to the RDI treatments. When irrigation was restored in stage III, $\Psi_{\mathrm{s}}$ for RDI trees recovered to values close to those found in $\mathrm{C}$ trees. 
The increasing evaporative demand during postharvest caused that $\Psi_{\mathrm{s}}$ thresholds were attained and, thus, irrigation was applied accordingly. However, in the last year of the experiment (2010), the $\Psi_{\mathrm{s}}$ threshold for triggering irrigation in the RDI II treatment was never achieved and irrigation water was not applied (only the minimum amount of water with the fertilizer needs). The lowest $\Psi_{\mathrm{s}}$ values measured for C, RDI I and RDI II trees were, respectively, $-0.9,-2.0$ and $-2.4 \mathrm{MPa}$, depending on the year, although they were always reached at postharvest. These reflect conditions of absence of water stress, moderate and severe water stress, respectively (Remorini and Massai, 2003) and followed the same trend as soil water content in the different treatments.

Tree functioning was affected by water deprivation, as lower $\mathrm{P}_{\mathrm{n}}$ and $\mathrm{g}_{\mathrm{s}}$ values were observed under RDI treatments. This indicates that peach trees regulated their transpiration when subjected to water constraints (Girona et al., 1993; Ruiz-Sánchez et al., 2010), which is a common response of cultivated plants grown in Mediterranean climates (Schulze et al., 1972; Tenhunen et al., 1982; Pereira et al., 1986). A delay in the recovery of these functions was observed when irrigation was restored, as previously reported for other species (Torrecillas et al., 1999; Romero et al., 2004). This progressive recovery after rewatering can be considered a mechanism for maintaining leaf productivity and promoting leaf rehydration (Torrecillas et al., 1999).

Vegetative growth (shoot elongation, TCSA and pruning weight) was significantly reduced by irrigation with holding during stage II and postharvest, when compared to full irrigation conditions, as previously reported in similar studies (Bradford and Hsiao, 1982; Pérez-Pastor, 2001; Girona et al., 2003, 2005; López et al., 2008; Abrisqueta et al., 2010). This reduction seems to be directly related to the amount of water applied and is desirable for high-density orchards in order to optimize tree-light interception and improve economic revenues (Chalmers et al., 1981) due to lower operational costs.

In contrast, fruit growth was not reduced by water deprivation during stage II and postharvest. In fact, similar crop loads and yields were observed for RDI treatments when compared to $\mathrm{C}$ trees. This trend was maintained for the three years of the experiment. As a consequence, greater water productivity values were observed for the RDI treatments. Moreover, flower density and fruit set were slightly affected by RDI, 
suggesting that withholding water during postharvest did not cause any carry-over effect from year to year in a medium-term. This is in contrast with previous results (Girona et al., 2003) and can be explained by the greater soil depth and rainfall amounts occurred in our study.

Fruit distribution into marketable categories was unaffected by the irrigation treatment in any of the three years studied. These results are in disagreement with those reported by Conejero et al. (2007) for an early-maturing peach cultivar and by Alcobendas et al. (2013) for the same cultivar used in the current study. In the latter case, this contradiction might have been caused by the low crop load of the trees studied by Alcobendas et al. (2013), since high-loaded peach trees are more sensitive to water stress than those with low loads (Marsal et al., 2006; López et al., 2008). In our case, fruit destination caused a lower thinning in 2009 with respect to those of 2008 and 2010; consequently, $\Psi_{\mathrm{s}}$ values in 2009 were lower compared to those from the other years. In spite of this, water deprivation did not limit crop yield and marketable fruit size in the following year.

Improvements in fruit taste and quality are expected for RDI treatments due to increasings in SSC (Crisosto et al., 1994; Mpelasoka et al., 2000; López et al., 2011). Our results showed significant increasings in SSC for RDI treatments. Moreover, the ratio SSC/TA was greater for the fruits from the RDI treatments, hence this may affect taste perception (Crisosto et al., 1997; Scandella et al., 1997) by the consumer, which might have implications in buying decisions.

Finally, when comparing the combination of deficit irrigation during stage II and postharvest with fully irrigated trees, higher water savings were achieved, up to $65 \%$ in the case of RDI II for 2010. Hence, water savings depended on the $\Psi_{\mathrm{s}}$ threshold used and on climate conditions of the specific year. As reported by Girona et al. (2003), the level of water savings during postharvest was much greater than that of stage II, making postharvest a more appealing period than stage II for saving applied water. Several studies in other fruit-tree crops support these findings (Romero et al., 2004; Girona et al., 2006; Pérez-Pastor et al., 2009).

In summary, the major effects of RDI in this study were related to reductions in vegetative growth and improvements in fruit quality, whereas crop yield remained 
unaffected. From a viewpoint of sustainability of water resources, RDI allowed to reduce water consumption around 55\%, in average for the three studied years. However, these water savings were dependent on the $\Psi_{\mathrm{s}}$ threshold used, which must be explored in order to adapt them to the specific conditions of the site where they will be used (Ghrab et al., 2013).

\subsection{Conclusions}

Our results proved that using $\Psi_{\mathrm{s}}$ thresholds for scheduling irrigation in mid-late maturing peach trees under Mediterranean conditions is a viable option to save water without compromising yield. In this experiment, a $\Psi_{\mathrm{s}}$ threshold of $-1.8 \mathrm{MPa}$ at stage II of fruit development and $-2.0 \mathrm{MPa}$ at postharvest induced water savings up to $68 \%$ over the growing season when compared to full irrigation based on the water balance method.

Tree vegetative growth was restricted by RDI and this trend was maintained over the whole experiment. This feature may be of interest for growers since lower costs for pruning operations (reductions in work-time) are expected, as well as a better distribution of light interception by the tree canopy.

Fruit set, crop load and yield were unaffected by the RDI strategies considered in this study. In addition, chemical attributes indicated a slight improvement in fruit quality under the RDI treatments. However, carry-over effects from year to year need to be assessed for the long-term. Hence, further experiments are required in order to evaluate the sustainability of the strategies considered in this study as well as to adapt the $\Psi_{\mathrm{s}}$ thresholds to other soil and climate conditions.

\section{Acknowledgements}

This study was supported by IRRIQUAL (EU-FP6-FOOD-CT-2006-023120) and SIRRIMED (KBBE-2009-1-2-03, PROPOSAL N॰245159) projects. We are also grateful to two SENECA projects (05665/PI/07 and 11872/PI/09) and CONSOLIDER INGENIO 2010 (MEC CSD2006-0067) and CICYT (AGL2010-17553) projects for providing funds to finance this research. 


\subsection{References}

Abrisqueta, I., Tapia, L.M., Conejero, W., Sánchez-Tobirio, M.I., Abrisqueta, J.M., Vera, J., Ruiz-Sánchez, M.C., 2010. Response of early-peach [Prunus persica (L.)] trees to deficit irrigation. Span. J. Agric. Res. 8(S2), S30-S39.

Alcobendas, R., Mirás-Avalos, J.M., Alarcón, J.J., Nicolás, E., 2013. Effects of irrigation and fruit position on size, colour, firmness and sugar contents of fruits in a mid-late maturing peach cultivar. Sci. Hort. 164, 340-347.

Allen, R.G., Pereira, L.S., Raes, D., Smith, M., 1998. Crop evapotranspiration. Guidelines for computing crop water requirements. FAO Irrigation and Drainage paper No 56, Rome, Italy. 300 pp.

Artés, F., Escriche, A.J., Martínez, J.A., Marín, J.G., 1993. Quality factors in four varieties of melon (Cucumis melo L.). J. Food Qual. 16, 91-100.

Besset, J., Génard, M., Girard, T., Serra, V., Bussi, C., 2001. Effect of water stress applied during the final stage of rapid growth on peach trees (cv. "Big-Top"). Sci. Hort. 91, 289-303.

Bonet, L., Ferrer, P., Castel, J.R., Intrigliolo, D.S., 2010. Soil capacitance sensors and stem dendrometers. Useful tools for irrigation scheduling of commercial orchards? Span. J. Agric. Res. 8, 852-865.

Bradford, K.J., Hsiao, T.C., 1982. Physioogical responses to moderate stress, in: Lange, O.L., Nobel, P.S., Osmond, C.B., Ziegler, H. (Eds.), Physiological Plant Ecology II. Water relations and carbon assimilation. Encyclopedia of Plant Physiology vol. 12B, Springer-Verlag, Berlin, pp. 264-324.

Buendía, B., Allende, A., Nicolás, E., Alarcón, J.J., Gil, M.I., 2008. Effect of regulated deficit irrigation and crop load on the antioxidant compounds of peaches. J. Agric. Food Chem. 56, 3601-3608.

Chalmers, D.J., Mitchell, P.D., Van Heek, L., 1981. Control of peach tree growth and productivity by regulated water supply, tree density and summer pruning. J. Am. Soc. Hort. Sci. 106, 307-312.

Crisosto, C.H., Johnson, R.S., Luza, J.G., Crisosto, G.M., 1994. Irrigation regimes affect fruit soluble solids concentration and rate of water loss of "O'Henry" peaches. HortScience 29, 1169-1171.

Crisosto, C.H., Johnson, R.S., DeJong, T., Day, K.R., 1997. Orchard factors affecting postharvest stone-fruit quality. HortScience 32, 820-823.

Conejero, W., Alarcón, J.J., García-Orellana, Y., Nicolás, E., Torrecillas, A., 2007. Evaluation of sap flow and trunk diameter sensors for irrigation scheduling in early maturing peach trees. Tree Physiol. 27, 1753-1759.

Conejero, W., Mellisho, C.D., Ortuño, M.F., Moriana, A., Moreno, F., Torrecillas, A., 2011. Using trunk diameter sensors for regulated deficit irrigation scheduling in early maturing peach trees. Environ. Exp. Bot. 71, 409-415. 
Fereres, E., Soriano, M.A., 2007. Déficit irrigation for reducing agricultural water use. J. Exp. Bot. 58, 147-159.

Fernández, J.E., Cuevas, M.V., 2010. Irrigation scheduling from stem diameter variations: A review. Agric. Forest Meteorol. 150, 135-151.

Ghrab, M., Masmoudi, M.M., Ben Mimoun, M., Ben Mechlia, N., 2013. Plant- and climate-based indicators for irrigation scheduling in mid-season peach cultivar under contrasting watering conditions. Sci. Hort. 158, 59-67.

Girona, J., Mata, M., Arbonés, Alegre, S., Rufat, J., Marsal, J., 2003. Peach tree response to single and combined regulated deficit irrigation regimes under shallow soils. J. Am. Soc. Hort. Sci. 128, 432-440.

Girona, J., Mata, M., del Campo, J., Arbonés, A., Bartra, E., Marsal, J., 2006. The use of midday leaf water potential for scheduling deficit irrigation in vineyards. Irrig. Sci. 24, 115-127.

Girona, J., Mata, M., Goldhammer, D.A., Jonson, R.S., DeJong, T.M., 1993. Patterns of soil and tree water status and leaf functioning during regulated deficit irrigation scheduling in peach. J. Am. Soc. Hort. Sci. 118, 580-586.

Girona, J., Mata, M., Marsal, M., 2005. Regulated deficit irrigation during kernel filling period and optimal irrigation rates in almond. Agric. Water Manage. 75, 152167.

Goldhamer, D.A., Fereres, E., 2001. Irrigation scheduling protocols using continously recorded trunk diameter measurements. Irrig. Sci. 20, 115-125.

Goldhamer, D.A., Fereres, E., 2004. Irrigation scheduling of almond trees with trunk diameter sensors. Irrig. Sci. 23, 11-19.

Goldhamer, D.A., Salinas, M., 2000. Evaluation of regulated deficit irrigation on mature orange trees grown under high evaporative demand. Proceedings of the International Society of Citriculture (ISC), IX Congress, Orlando, FL, USA, pp. 227-231.

González-Altozano, P., Castel, J.R., 2000. Regulated deficit irrigation in 'Clementina de Nules’ citrus trees II. Vegetative growth. J. Hortic. Sci. Biotechnol. 75, 388-392.

Intrigliolo, D., Castel, J.R., 2006. Performance of various water stress indicators for prediction of fruit size response to deficit irrigation in plum. Agric. Water Manage. 83, 173-180.

López, G., Arbonés, A., Del Campo, J., Mata, M., Vallverdú, X., Girona, J., Marsal, J., 2008. Response of peach trees to regulated deficit irrigation during stage II of fruit development and summer pruning. Span. J. Agric. Res. 6, 479-491.

López G., Behboudian, M.H., Echeverría, G., Girona, J., Marsal, J., 2011. Instrumental and sensory evaluation of fruit quality for 'Ryan's Sun' peach grown under deficit irrigation. HorTechnology 21(6), 712-719. 
MAGRAMA, 2015. Anuario de estadística Avance 2014. Ministerio de Agricultura, Alimentación y Medio Ambiente.

Marsal, J., López, G., Girona, J., 2006. Recent advances in regulated deficit irrigation (RDI) in woody perennials and future perspectives. Aust. Nut. Grower 20, 4-8.

Moreno, F., Fernández, J.E., Clothier, B.E., Green, S.R., 1996. Transpiration and root water uptake by olive trees. Plant Soil 184, 85-96.

Moriana, A., Orgaz, F., Fereres, E., Pastor, M., 2003. Yield responses of a mature olive orchard to water deficits. J. Am. Soc. Hort. Sci. 128, 425-431.

Moriana, A., Giron, I., Martín-Palomo, M.J., Conejero, W., Ortuño, M.F., Torrecillas, A., Moreno, F., 2010. New approach for olive trees irrigation scheduling using trunk diameter sensors. Agric. Water Manage. 97, 1822-1828.

Mpelasoka, B.S., Behboudian, M.H., Dixon, J., Neal, S.M., Caspari, H.W., 2000. Improvement of fruit quality and storage potential of "Braeburn" apple through deficit irrigation. J. Hort. Sci. Biotechnol. 75, 615-621.

Myers, B.J., 1988. Water stress integral a link between short-term stress and long term growth. Tree Physiol. 4, 315-323.

Naor, A., Cohen, S., 2003. Sensitivity and variability of maximum trunk, shrinkage, midday stem water potential, and transpiration rate in response to withholding irrigation from field-grown apple trees. HortScience 38, 547-551.

Pereira, L.S., Tenhunen, J.D., Lange, O.L., Beyschalag, W., Meyer, A., David, M.M., 1986. Seasonal and diurnal patterns in leaf gas exchange of Eucalyptus globulus trees growing in Portugal. Can. J. Forest Res. 16, 177-184.

Pérez-Pastor, A., 2001. Estudio agronómico y fisiológico del albaricoquero en condiciones de infradotación hídrica. Ph.D. Thesis. Universidad Politécnica de Cartagena, Murcia, Spain.

Pérez-Pastor, A., Domingo, R., Torrecillas, A., Ruiz-Sánchez, M.C., 2009. Response of apricot trees to deficit irrigation strategies. Irrig. Sci. 27, 231-242.

Remorini, D., Massai, R., 2003. Comparison of water status indicators for young peach trees. Irrig. Sci. 22, 39-46.

Romero, P., Botía, P., García, F., 2004. Effects of regulated deficit irrigation under subsurface drip irrigation conditions on water relations of mature almond trees. Plant Soil 260, 155-168.

Ruiz-Sánchez, M.C., Domingo, R., Castel, J.R., 2010. Review. Deficit irrigation in fruit trees and vines in Spain. Span. J. Agric. Res. 8(S2), S5-S20.

Scandella D., Kraeutler E., Vénien S., 1997. Anticiper la qualité gustative des pêches et nectarines. Infos CTIFL 129, 16-19. [In French].

Schulze, E.D., Lange, O.L., Buschbom, U., Kappen, L., Evenari, M., 1972. Stomatal responses of intact growing plants to changes in humidity. Planta 108, 259-270. 
Shackel, K.A., Buchner, R.P., Connell, J.H., Edstrom, J.P., Fulton, A.E., Holtz, B.A., Lampinen, B.D., Reil, R.O., Stewart, W.L., Viveros, M.A., 2010. Midday stem water potential as a basis for irrigation scheduling. $5^{\text {th }}$ National Decennial Irrigation Conference Proceedings. Phoenix, Arizona, USA. IRR109993 (doi: 10.13031/2013.35841).

Tenhunen, J.D., Lange, O.L., Jahner, D., 1982. The control by atmospheric factors and water-stress of midday stomatal closure in arbutus-unedo growing in a natural macchia. Oecologia 5, 165-169.

Torrecillas, A., Galego, R., Pérez-Pastor, A., Ruiz-Sánchez, M.C., 1999. Gas exchange and water relations of young apricot plants under drought conditions. J Agric Sci $132,445-452$.

Turner, N.C., 1981. Techniques and experimental approaches for the measurement of plant water status. Plant Soil 58, 339-366. 


\section{EFFECTS OF REGULATED DEFICIT IRRIGATION ON PHYSIOLOGY, YIELD AND FRUIT QUALITY IN APRICOT TREES}





\title{
8. EFFECTS OF REGULATED DEFICIT IRRIGATION ON PHYSIOLOGY, YIELD AND FRUIT QUALITY IN APRICOT TREES
}

\begin{abstract}
Scarce water resources mainly in arid and semiarid areas caused an increasing interest for applying irrigation techniques aiming to reduce water spends. The effects of regulated deficit irrigation (RDI) were studied on the performance of 9 year old apricottrees (Prunus armeniaca L. cv. "Búlida") in Murcia (SE Spain), during three consecutive growing seasons (2008-2010). Two irrigation treatments were established: a control treatment, irrigated to fully satisfy crop water requirements $\left(100 \% \mathrm{ET}_{\mathrm{c}}\right)$ and an RDI treatment, that reduced the amount of applied irrigation water to: a) $40 \%$ of $E_{c}$ at flowering and first stage of fruit growth; b) $60 \%$ of $\mathrm{ET}_{\mathrm{c}}$ during the second stage of fruit growth and c) $50 \%$ and $25 \%$ of $\mathrm{ET}_{\mathrm{c}}$ during the late postharvest period. Stem water potential $\left(\Psi_{\mathrm{s}}\right)$, gas exchanges, trunk cross-sectional area, fruit diameter, yield and fruit quality parameters were determined for each treatment. The results indicated that the apricot tree is an appropriate species to apply RDI thanks to the clear separation between its vegetative and reproductive growths and its ability to recover the fruit diameter reduction suffered during RDI application. Furthermore, some qualitative characteristics of the fruits, such as the level of soluble solids, sweetness/acidity relation and fruit colour, are enhanced. These reasons, as well as average irrigation water savings of $33 \%$, emphasize the RDI strategies as a possible solution in areas with water shortages, like the south-eastern region of Spain.
\end{abstract}

Keywords: fruit growth, photosynthesis; Prunus armeniaca L.; regulated deficit irrigation; stomatal conductance; water stress.

\subsection{Introduction}

The apricot tree (Prunus armeniaca L.) is one of the most important fruit species worldwide since its fruit is highly appreciated by consumers (Roussos et al., 2011). Apricot world production is about 2.5 million t per year and Turkey is the leading producing country, according to the Food and Agriculture Organization, with approximately 676,000 t (FAO, 2013). Spain produces about 132,000 t from a cultivation area of 20,000 ha. In the Murcia Region (SE Spain) almost all the cultivated area $(\approx 10,000$ ha in 2014$)$ is irrigated. This cultivation area yielded 79,000 $\mathrm{t}$ in 2014 
(60\% of apricot production in Spain) (MAGRAMA, 2015). The most important Spanish apricot cultivar is "Búlida", which represents $66 \%$ of the total production in the Murcia Region (CARM, 2009).

Apricot drought tolerance is mainly based on avoidance mechanisms, such as stomatal control, epinasty and limitation transpiration by reducing leaf area (RuizSánchez et al., 2000a), together with some degree of osmotic adjustment in young apricot trees although this adjustment is not observed in adult trees (Ruiz- Sánchez et al., 2007). However, apricot trees are highly sensitive to drought stress at particular phenological stages, such as stage III of fruit growth and during the 2 months after harvest (early postharvest) (Torrecillas et al., 2000; Pérez- Pastor et al., 2009).

Mediterranean regions are characterised by the shortage on water resources. This situation is aggravated by the strong competition for water between agriculture and other non-agricultural users like industry or increasing population. It is therefore necessary to develop and implement techniques in order to optimize agricultural water use without affecting crop yields. One of the most promising techniques to attain this objective is the use of Regulated Deficit Irrigation (RDI).

RDI was developed in Australia in peach orchards based on the use of water stress to control vigour and competition for photoassimilates between fruits and other plant organs (Chalmers et al., 1981). This technique consists of applying water in quantities below those necessary to fully satisfy crop evapotranspiration $\left(\mathrm{ET}_{\mathrm{c}}\right)$ requirements during certain periods of the crop cycle when yield and crop quality are hardly affected, applying all the water needed during the rest of the cycle, especially at critical periods when the yield and/or quality would be greatly affected by a lack of water. RDI is normally applied when reproductive growth is relatively slow and when vegetative growth and other plant processes may be affected, such effects frequently being translated into improved fruit quality (Ruiz-Sanchez et al., 2010).

RDI has been successfully used, maintaining yield and fruit quality, in many fruit species (Buendía et al., 2008; López et al., 2008), citrus species (GonzálezAltozano and Castel, 2000) and olives (Moriana et al., 2010). Researches about RDI strategies applied to apricot trees reported benefits such as higher values of total soluble 
solids, titratable acidity and hue angle in apricot fruits grown under RDI (Pérez-Pastor et al., 2009, 2014).

The aim of this paper was to evaluate the effects of RDI on plant-water relations, yield and fruit quality in adult apricot trees (Prunus armeniaca L. cv. "Búlida") over three consecutive growing seasons (2008-2010) under the climatic conditions of SE Spain.

\subsection{Materials and methods}

\section{Description of the study site and plant material}

The experiment was conducted over three consecutive years (2008-2010) in a 1ha plot of a commercial orchard located in Mula valley, Murcia, SE Spain $\left(37^{\circ} 55^{\prime} \mathrm{N}\right.$, $1^{\circ} 25^{\prime} \mathrm{W}, 360 \mathrm{~m}$ above sea level).

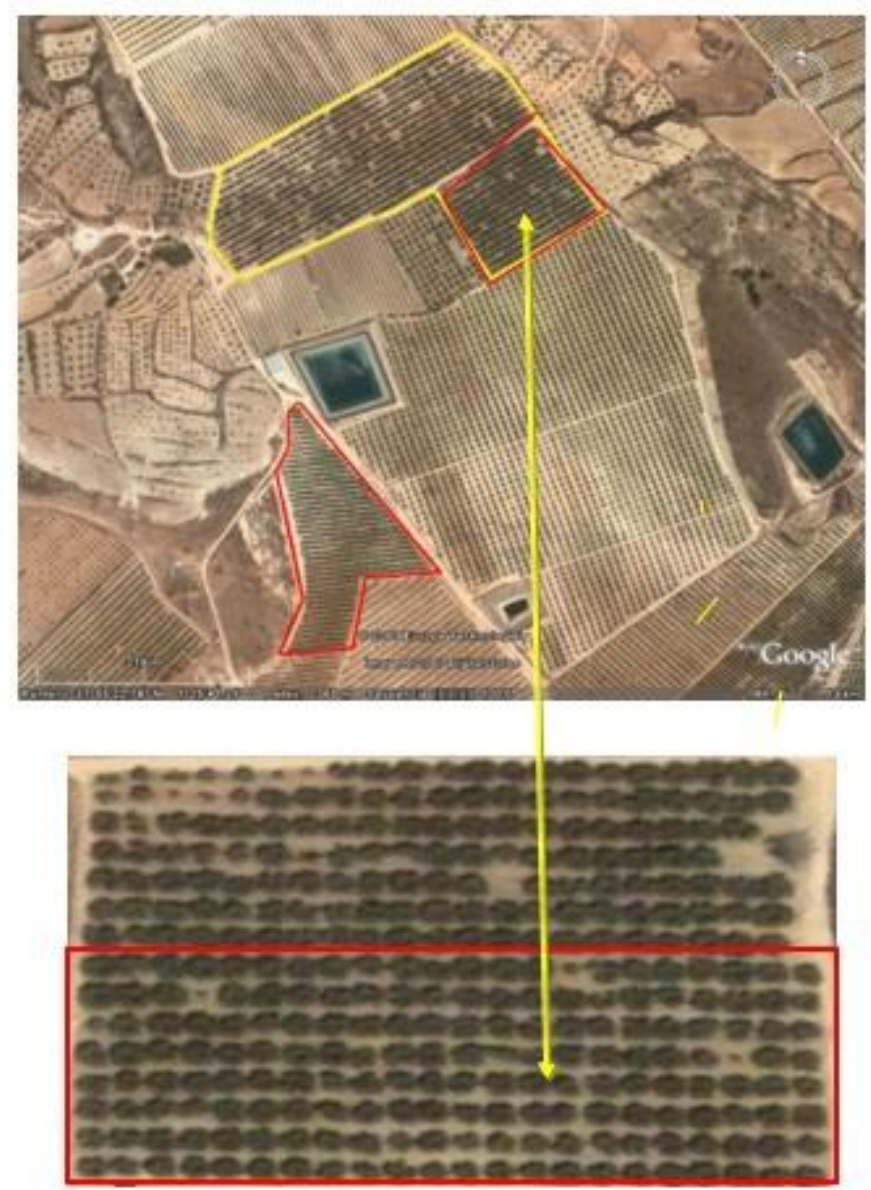

Picture 8.1. Plot location. (Source: Google Maps). 
The soil is clay-loam textured and classified as a Xeric Torriorthent (Soil Survey Staff, 1999). It is highly calcareous $(\mathrm{pH}=7.8)$, with low organic matter content and cationic exchange capacity. The available water capacity is about $0.31 \mathrm{~m}^{3} \mathrm{~m}^{-3}$. The climate of the region is semiarid Mediterranean with hot and dry summers; annual evaporation calculated from reference crop evapotranspiration $\left(\mathrm{ET}_{0}\right)$ and rainfall during the growing season were, respectively, 1,055 and $318 \mathrm{~mm}$ in the first year of experiments (2008), 1,064 and $568 \mathrm{~mm}$ in 2009 and 991 and $388 \mathrm{~mm}$ in 2010.

The plant material consisted of apricot trees (Prunus armeniaca L.cv. "Búlida") grafted on Real fino apricot rootstock, and planted in 1999. Spacings were 8 m x 6 m. Trees were drip irrigated using one drip irrigation line for each row, with five emitters per tree (each delivering $4 \mathrm{~L} \mathrm{~h}^{-1}$ ).

\section{Irrigation treatments and experimental design}

Crop irrigation requirements were scheduled weekly according to daily $\mathrm{ET}_{0}$, calculated using the Penman-Monteith equation (Allen et al., 1998), and a local crop factor based on the time of the year (Abrisqueta et al., 2001): 0.5 February, 0.75 March, 0.8 April, 0.9 May, 0.6 June, 0.5 July-November. The correction coefficient for ground cover was obtained according to Fereres and Goldhamer (1990). All trees received the

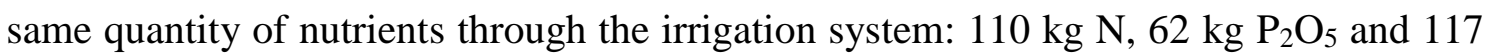
$\mathrm{kg} \mathrm{K}_{2} \mathrm{O}$ ha $^{-1}$ year $^{-1}$. Pest control was that commonly used by growers, and no weeds were allowed to develop within the orchard. A total of 192 trees were used in this study. The experiment was laid out in completely randomized blocks with 4 replications (24 trees each). The four central trees from each replication were used for measurements, and the other trees acted as guard.

Two irrigation treatments were applied: i) Control (C), daily irrigated to fully satisfy the estimated crop evapotranspiration $\left(\mathrm{ET}_{\mathrm{c}}\right)$ and ii) $\mathrm{RDI}$ irrigated at $100 \% \mathrm{ET}_{\mathrm{c}}$ during the critical periods (stage III of fruit growth and 2 months after harvest) and subjected to water shortage during the non-critical periods of crop development by reducing the amount of applied irrigation water to: a) $40 \%$ of $\mathrm{ET}_{\mathrm{c}}$ from flowering until the end of the first stage of fruit growth; b) $60 \%$ of $\mathrm{ET}_{\mathrm{c}}$ during the second stage of fruit growth and c) $50 \%$ and $25 \%$ of $\mathrm{ET}_{\mathrm{c}}$ during the late postharvest period (that starts 60 days after harvesting), for the first 30 days and until the end of tree defoliation, respectively (Figure 8.1). 
This distribution of applied water during non-critical periods was based on studies by Torrecillas et al. (2000). The irrigation water was considered of good quality with a very low electrical conductivity $\left(0.6 \mathrm{dS} \mathrm{m}^{-1}\right)$.

Irrigation was automatically controlled by a head unit programmer and the amounts of water applied for each irrigation treatment were measured with in-line flowmeters placed in each experimental plot

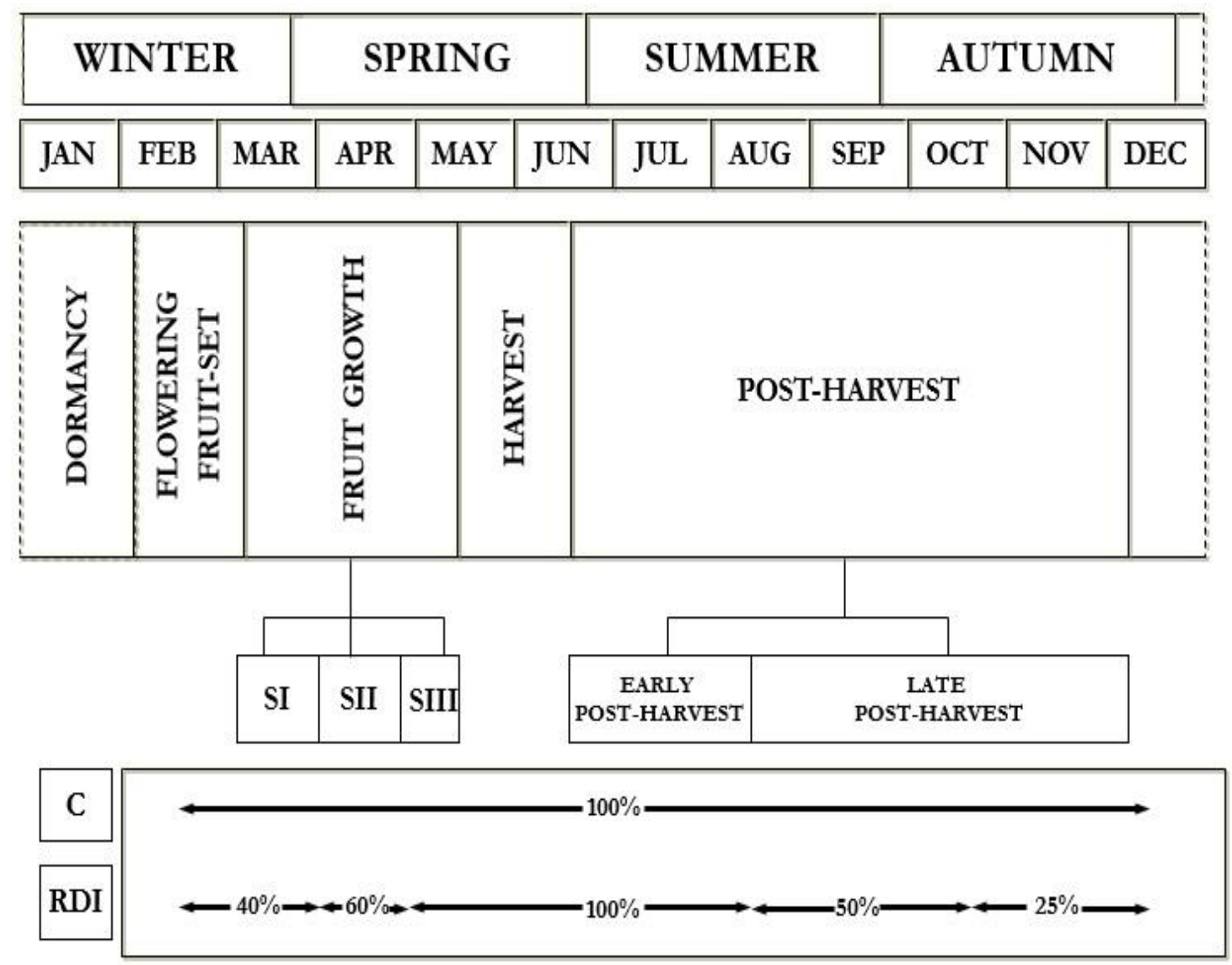

Figure 8.1. Percentages of crop evapotranspiration $\left(\mathrm{ET}_{\mathrm{c}}\right)$ applied to the regulated deficit irrigation (RDI) treatment for each phenological stage of "Búlida" apricot trees over the experimental period.

\section{Measurements}

\section{Climate data}

Climate data were recorded at an automatic weather station placed within the experimental orchard. Data on air temperature (maximum, minimum and average), solar radiation, air relative humidity, rainfall and wind speed $2.5 \mathrm{~m}$ above the soil surface, were collected every $15 \mathrm{~min}$.

These data were used to calculate $\mathrm{ET}_{0}$ and to establish crop water requirements. The evolution of these parameters over the study period is shown in figure 8.2. 


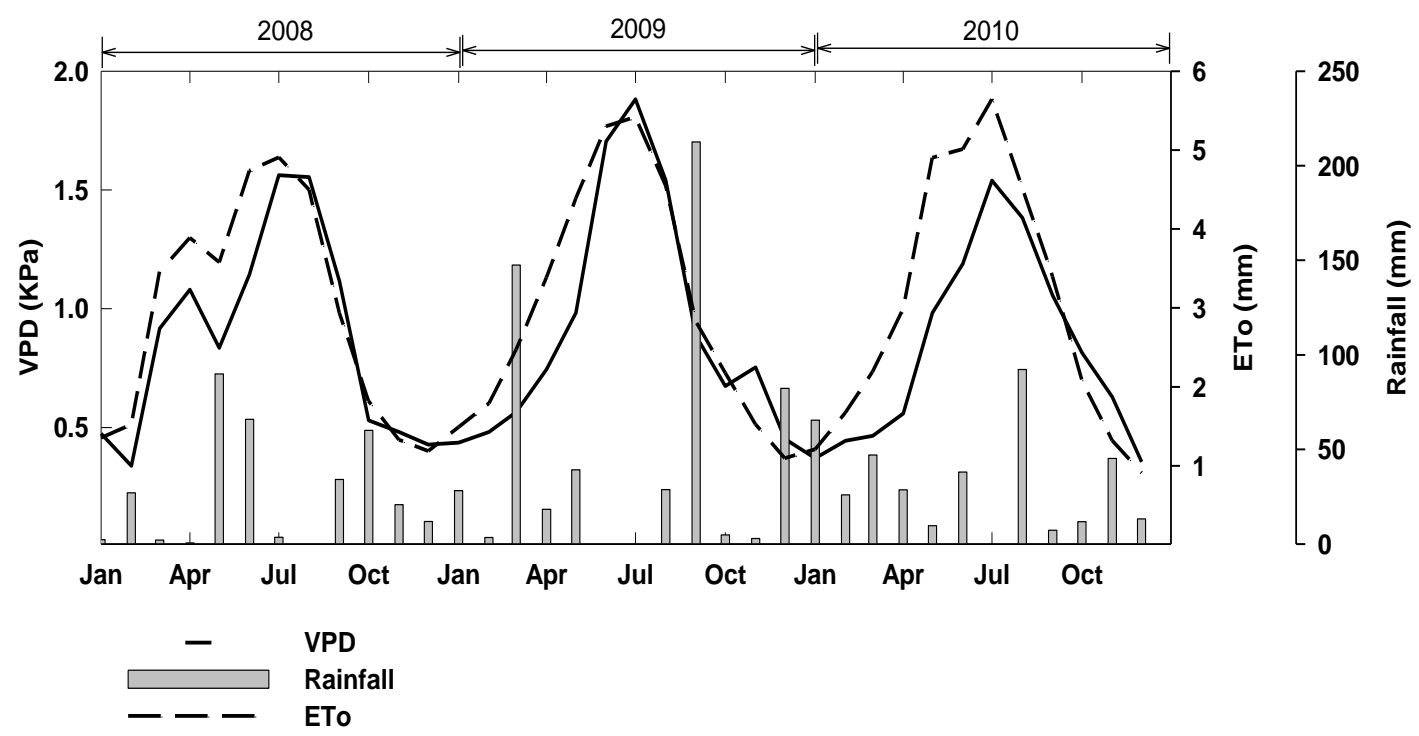

Figure 8.2. Reference evapotranspiration $\left(\mathrm{ET}_{0}\right)$, rainfall $(\mathrm{mm})$ and $\mathrm{VPD}(\mathrm{KPa})$ at the experimental site. Monthly values from data collected during 2008, 2009 and 2010.

\section{Soil water content}

The volumetric soil water content $\left(\theta_{\mathrm{v}}, \mathrm{m}^{3} \mathrm{~m}^{-3}\right)$ of the top $0.2 \mathrm{~m}$ of the soil profile was measured by time domain reflectometry (TDR) (Model 1502C, Tektronix Inc., OR), as described by Moreno et al. (1996). The $\theta_{\mathrm{v}}$ content of the soil from $0.2 \mathrm{~m}$ down to a maximum depth of $1.0 \mathrm{~m}$ was measured every $0.1 \mathrm{~m}$ using a neutron probe (Model 4300, Troxler Electronic Laboratories Inc., NC), in access tubes installed $1.0 \mathrm{~m}$ from the trees and beside the emitters. These probes were placed at $0.20 \mathrm{~m}$ from the emitter and next to TDR rods. Measurements using one neutron probe and TDR per experimental plot (4 replications per treatment) were taken in the morning, every 7 to 15 days, during the experimental period.

\section{Stem and leaf water potential}

Midday (12:00 h solar time) stem water potential $\left(\Psi_{\mathrm{s}}\right)$ was measured in one mature leaf per plant (4 trees per experimental plot), taken close to the trunk. Leaves were enclosed in a small black plastic bag covered with aluminium foil for at least $2 \mathrm{~h}$ before measurements were made with a pressure chamber (Soil Moisture Equip. Corp, model 3000, Santa Barbara, CA, USA). Leaf water potential $\left(\Psi_{1}\right)$ was measured in the same trees used for $\Psi_{\mathrm{s}}$ measurements, sampling one mature and sunny-exposed leaf per plant. The water potential measurements were made according to Scholander et al. (1965) and following the recommendations of Turner (1988). 
Gas exchange parameters

Net photosynthesis $\left(\mathrm{P}_{\mathrm{n}}\right)$ and stomatal conductance $\left(\mathrm{g}_{\mathrm{s}}\right)$ were measured at solar midday, in one fully expanded sun-facing leaf in the four central trees of each experimental plot per treatment, in the same days that stem water potential was recorded, using a field-portable photosynthesis system (LI-6400, Li-Cor, Lincoln, NE, USA).

\section{Vegetative and fruit growth}

Trunk diameter was measured annually during the dormancy stage in 96 trees per treatment with a sliding caliper, $0.20 \mathrm{~m}$ above the soil surface. Trunk cross-sectional area (TCSA) was calculated from these data.

Over the growing season, fruit diameter was measured perpendicularly to the fruit suture on 200 fruits per treatment (50 fruits per replication). Each sampling was carried out every 7-10 days, randomly measuring 12-13 fruits in the four inner trees per experimental plot using a digital caliper (Powerfix model Nr Z22855F, Milomex Ltd, Bedfordshire, UK).

\section{Yield and fruit quality}

At harvest, 200 fruits per treatment (50 fruits per experimental plot) were randomly selected for quality assessment. Skin and flesh colour, firmness, $\mathrm{pH}$, soluble solids content (SSC) and titratable acidity (TA) were evaluated as quality indices.

Colour values, on the surface (ground skin colour) and after peeling in the flesh, were measured with a Minolta chromameter (CR-300, Minolta, Ramsey, NJ) tristimulus colour analyzer calibrated with a white porcelain reference plate. The colour space coordinates $\mathrm{L}^{*}, \mathrm{a}^{*}$, and $\mathrm{b}^{*}$, hue angle $\left[\mathrm{H}^{\mathrm{o}}=\operatorname{arctg}\left(\mathrm{a}^{*} / \mathrm{b}^{*}\right)\right]$, and chroma $\left(\mathrm{a}^{* 2} / \mathrm{b}^{* 2}\right)^{1 / 2}$ were determined around the equatorial region in three different positions (with an average of nine times for each apricot). 


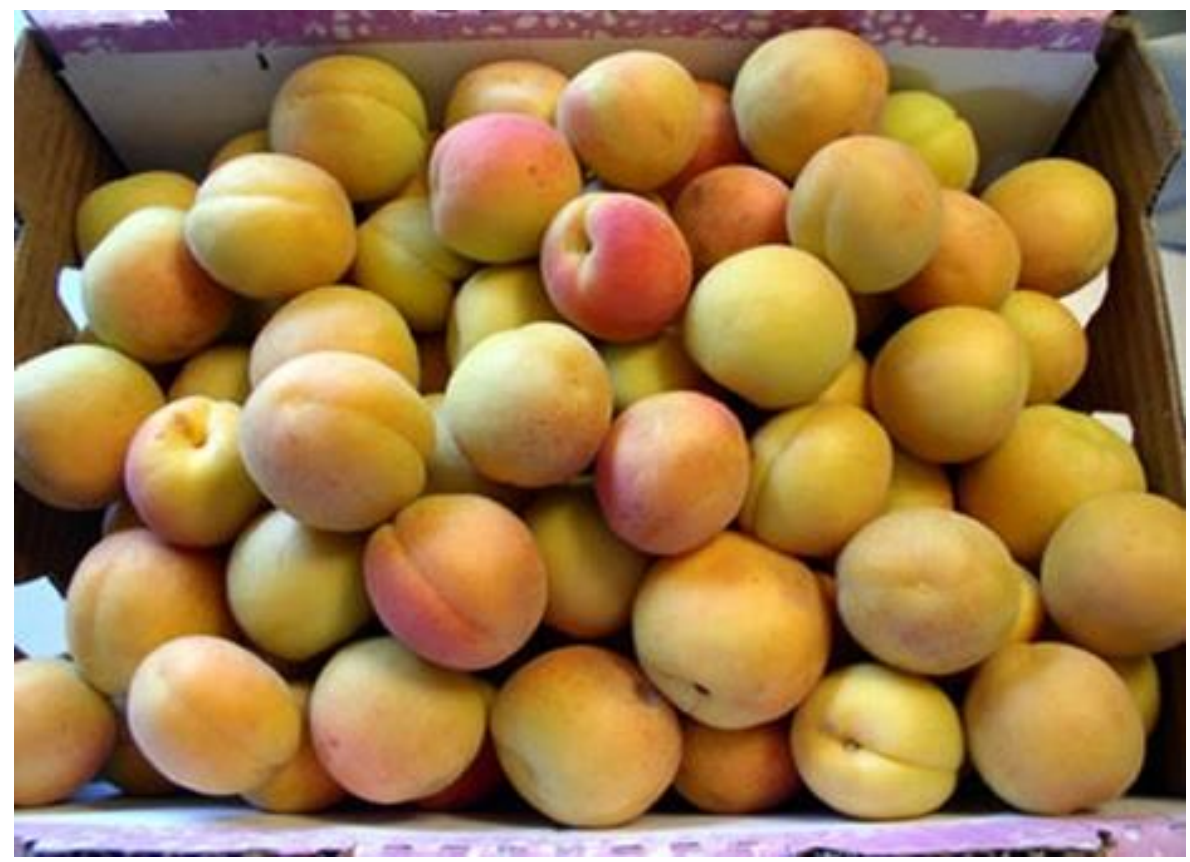

Picture 8.2. Recently harvested apricots (Own Source).

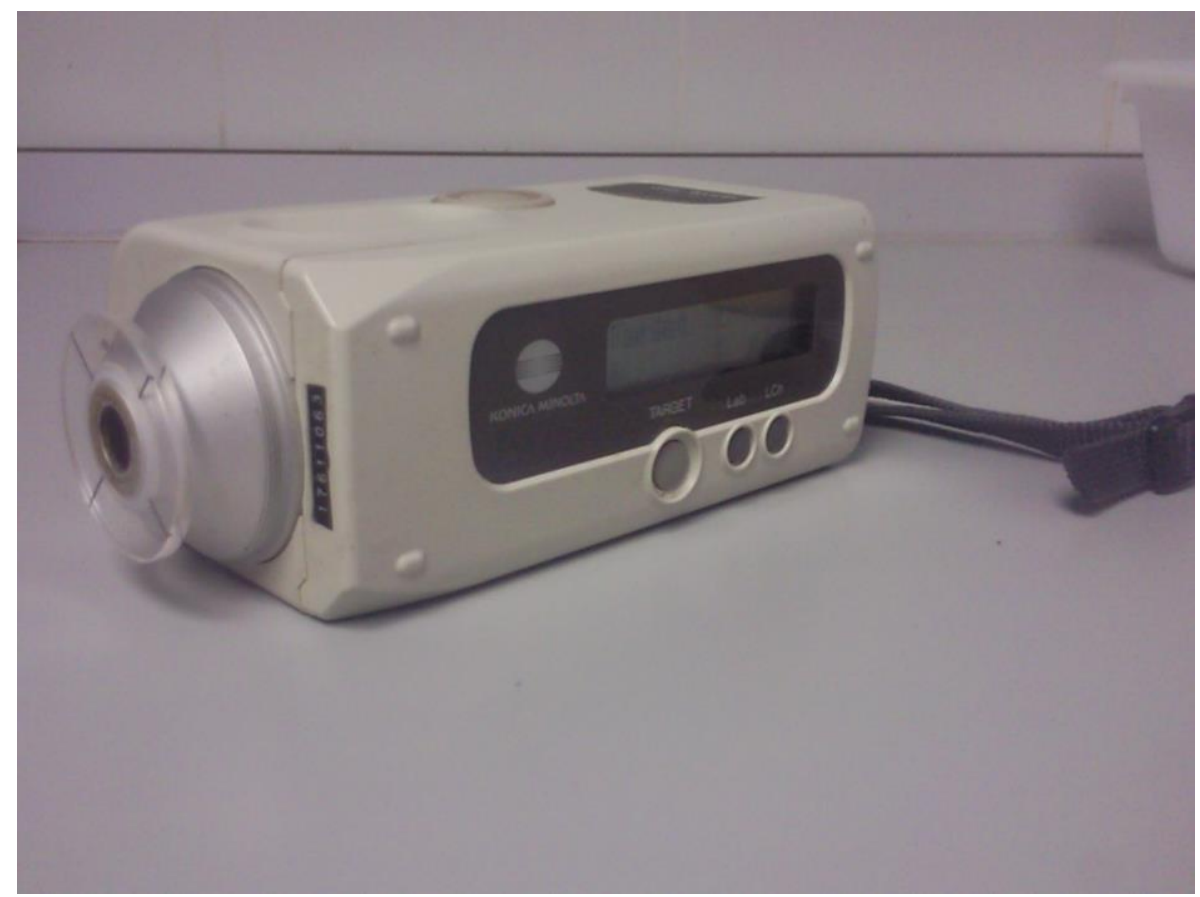

Picture 8.3. Minolta chromameter (Own Source).

Fruit firmness was evaluated using a Durofel penetrometer DFT100 (AgroTechnologie S.A., Paris, France). Juice was extracted from combined samples of longitudinal unpeeled slices. Total soluble solids concentration (SSC) was determined with a hand-held refractometer (Atago, Co., Japan); and values were expressed as ${ }^{\circ}$ Brix. Titratable acidity (TA) was measured by titration of $5 \mathrm{~mL}$ of juice with $0.1 \mathrm{~mol} \mathrm{~L}^{-1}$ $\mathrm{NaOH}$ to $\mathrm{pH} 8.1$ using an automatic titration system and expressed as g malic acid $\mathrm{L}^{-1}$ 
(AOAC, 1984). The maturity index was calculated as the $\mathrm{SSC} \mathrm{TA}^{-1}$ ratio. $\mathrm{pH}$ was measured using a pH-meter (Crison, Barcelona, Spain).

\section{Water stress integral and water productivity}

In order to evaluate the intensity of water stress, the water stress integral (MPadays) was calculated from the $\Psi_{\mathrm{s}}$ data according to the following equation (Myers, 1988):

$$
S_{\Psi}=\left|\sum_{i=0}^{i=t}\left(\bar{\Psi}_{i, i+1}-c\right) n\right|
$$

where:

$\Psi_{\mathrm{i}, \mathrm{i}+1}(\mathrm{MPa})$ is the average $\Psi_{\mathrm{s}}$ for each time interval

$c(\mathrm{MPa})$ is the value of the maximum $\Psi_{\mathrm{s}}$ in all seasons

$n$ is the number of days in the interval.

Water productivity was calculated as the ratio between yield and total irrigation water applied (Kijne et al., 2003), expressed in $\mathrm{Kg} \mathrm{m}^{-3}$.

\section{Statistical analysis}

Statistical analysis was performed as a weighted analysis of variance (ANOVA; statistical software IBM SPSS Statistics v. 21 for Windows, Chicago). The ShapiroWilk test was used to evaluate the normality of the data. Tukey's HSD test was used for mean separation. Unless otherwise stated, the significance level was $p \leq 0.05$.

\subsection{Results}

\section{Soil water content and irrigation}

Volumetric soil water content during the 2008-2010 period (Figure 8.3), from 0 to $1 \mathrm{~m}$ depth, in the $\mathrm{C}$ treatment was nearly constant, with values close to field capacity. In the RDI treatment, $\theta_{\mathrm{v}}$ decreased in all phenological periods before stage III of fruit growth, and recovered in this stage and early postharvest, when full irrigation was restored. 
During the late postharvest period, the $\theta_{\mathrm{v}}$ decreased because of the deficit irrigation applied. The soil moisture profile in the RDI treatment was characterized by the fact that during the water deficit periods, the $\theta_{\mathrm{v}}$ values at depths greater than $0.6 \mathrm{~m}$ were clearly below field capacity, indicating the non-existence of drainage (data not shown).

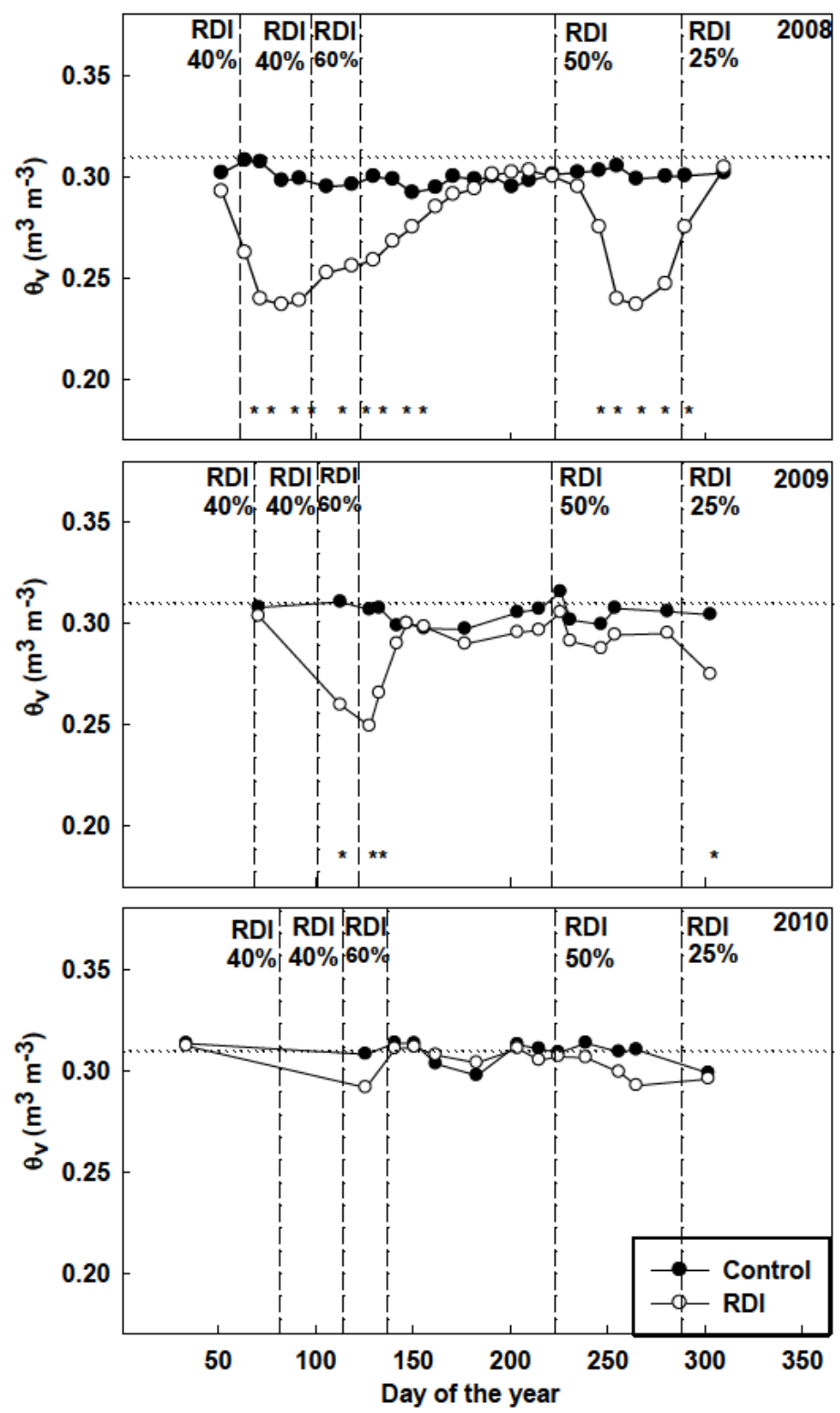

Figure 8.3. Volumetric soil water content down to $1 \mathrm{~m}$ depth $\left(\theta_{\mathrm{v}}\right)$, in C (closed circles) and RDI (open circles) treatments for the three growing seasons studied (2008-2010). Each data point is the mean of four values. Asterisks indicate significant differences between treatments $(p<0.05)$. The intervals between vertical lines from left to right represent the beginning of the different phenological stages for apricot trees during the experimental period. Horizontal lines represent soil field capacity. 
Significant differences in $\theta_{\mathrm{v}}$ values were observed between treatments during flowering and fruit set, stage I and II and late postharvest until the end of the experiment in 2008; the following year, these differences were only observed in stage II and at the beginning of stage III until the soil water content was recovered due to the restoration of full irrigation in the RDI treatment and in the last point of measurements in late postharvest; during 2010, no significant differences between treatments were detected, although slightly lower soil water contents were measured in stage II of fruit development and early post-harvest stages (Figure 8.3).

The amounts of water applied during the 2008 growing season were 574 and 325 $\mathrm{mm}$ in C and RDI, respectively. In 2009, these amounts were 437 and $342 \mathrm{~mm}$, whereas in 2010 they were 520 and $365 \mathrm{~mm}$ for C and RDI, respectively (Table 8.1). The average water saved over the three years in the RDI treatment was 57\% (flowering and fruit set), $45 \%$ (stage I of fruit growth), $41 \%$ (stage II of fruit growth) and 56\% during late postharvest period. Overall, for the entire growing season, RDI saved $33 \%$ water as compared to C.

Table 8.1. Cumulative applied water $(\mathrm{mm})$ during the different stages of fruit growth for the three studied growing seasons, in $\mathrm{C}$ and RDI trees.

\begin{tabular}{|c|c|c|c|c|c|c|c|c|c|}
\hline & & Dormancy & $\begin{array}{c}\text { Flowering } \\
\qquad \& \\
\text { Fruit set }\end{array}$ & $\begin{array}{c}\text { Stage } \\
\text { I }\end{array}$ & $\begin{array}{c}\text { Stage } \\
\text { II }\end{array}$ & $\begin{array}{c}\text { Stage } \\
\text { III }\end{array}$ & $\begin{array}{c}\text { Early } \\
\text { Post-Harvest }\end{array}$ & $\begin{array}{c}\text { Late } \\
\text { Post-Harvest }\end{array}$ & Total \\
\hline \multirow[t]{2}{*}{2008} & Control & 14.17 & 23.05 & 35.77 & 41.52 & 54.49 & 219.81 & 185.19 & 574.00 \\
\hline & RDI & 10.57 & 8.74 & 15.65 & 22.91 & 48.45 & 150.19 & 68.90 & 325.40 \\
\hline \multirow[t]{2}{*}{2009} & Control & 12.15 & 11.68 & 11.99 & 17.70 & 48.50 & 189.06 & 145.93 & 437.00 \\
\hline & RDI & 10.53 & 5.29 & 6.57 & 10.81 & 45.50 & 190.09 & 73.72 & 342.50 \\
\hline \multirow[t]{2}{*}{2010} & Control & 14.13 & 5.43 & 15.89 & 31.42 & 60.37 & 153.43 & 239.73 & 520.40 \\
\hline & RDI & 12.20 & 3.35 & 12.52 & 19.52 & 57.41 & 151.36 & 109.25 & 365.60 \\
\hline \multirow[t]{2}{*}{ 2008-2010 } & Control & 13.49 & 13.39 & 21.22 & 30.21 & 54.45 & 187.43 & 190.28 & 510.47 \\
\hline & RDI & 11.10 & 5.79 & 11.58 & 17.75 & 50.45 & 163.88 & 83.96 & 344.50 \\
\hline \multicolumn{2}{|c|}{ Water Savings (\%) } & 18 & 57 & 45 & 41 & 7 & 13 & 56 & 33 \\
\hline
\end{tabular}

\section{Plant water relations}

The leaf water potential in both treatments was not significantly different in any period, except for isolated dates (five in 2008 and one in 2010) (Figure 8.4). In the second year of experiments (2009), the values of leaf water potential were more negative than those from the first and last years, probably due to higher temperatures, especially in the summer months. 
The evolution of $\Psi_{\mathrm{s}}$ showed a decreasing trend in all treatments due to increased climate demand with the advent of warmer months (Figure 8.5). The RDI treatment induced significant reductions in $\Psi_{\mathrm{s}}$ in all the stages during which water deficit was imposed in 2008 and 2009. Nevertheless, during 2010, these differences were only significant in the postharvest period.

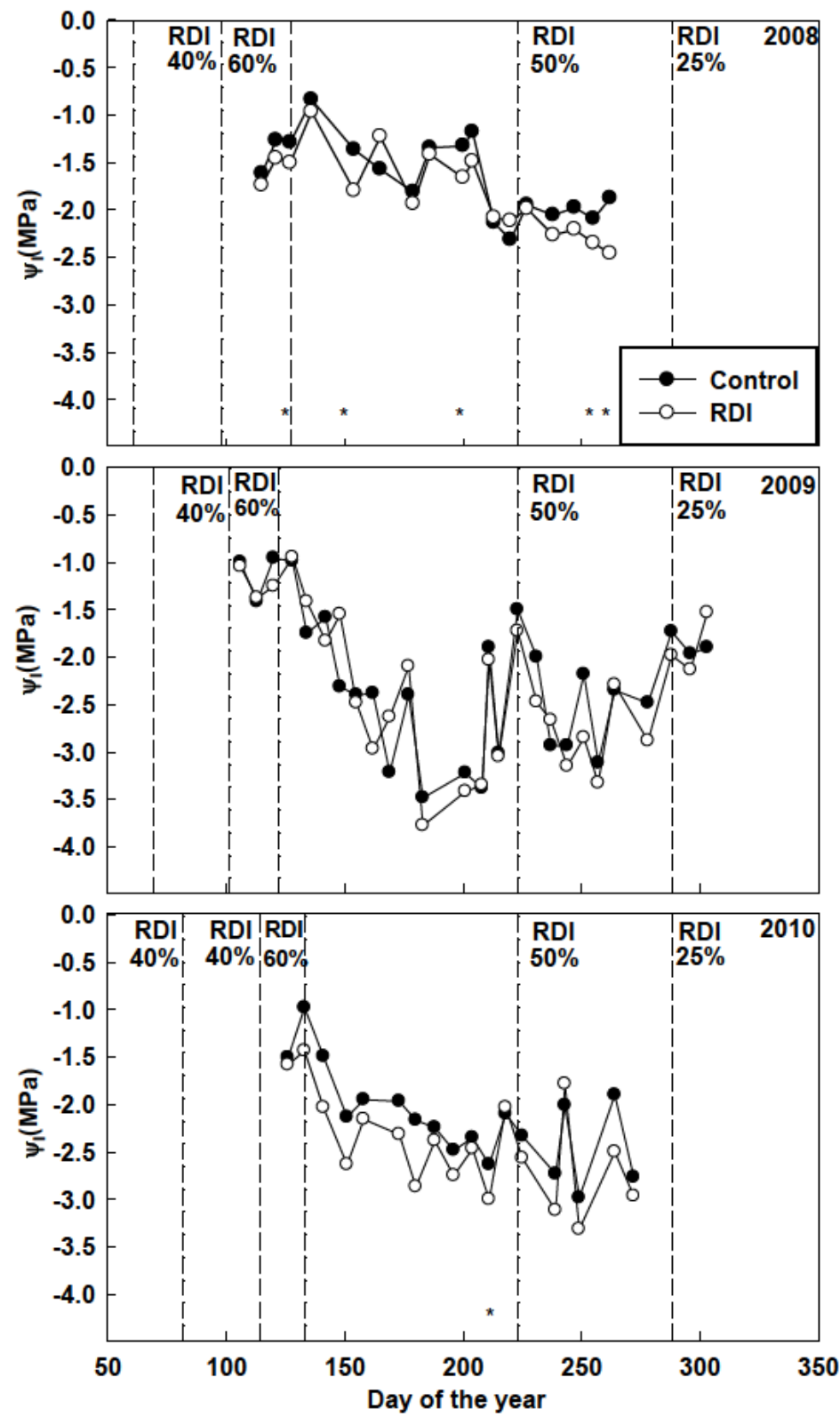

Figure 8.4. Midday leaf water potential in C (closed circles) and RDI (open circles) trees for the three studied growing seasons. Each data point is the mean of sixteen values. The intervals between vertical lines from left to right represent the beginning of the different phenological stages for apricot trees during the experimental period. Asterisks indicate significant differences between treatments $(p<0.05)$. 

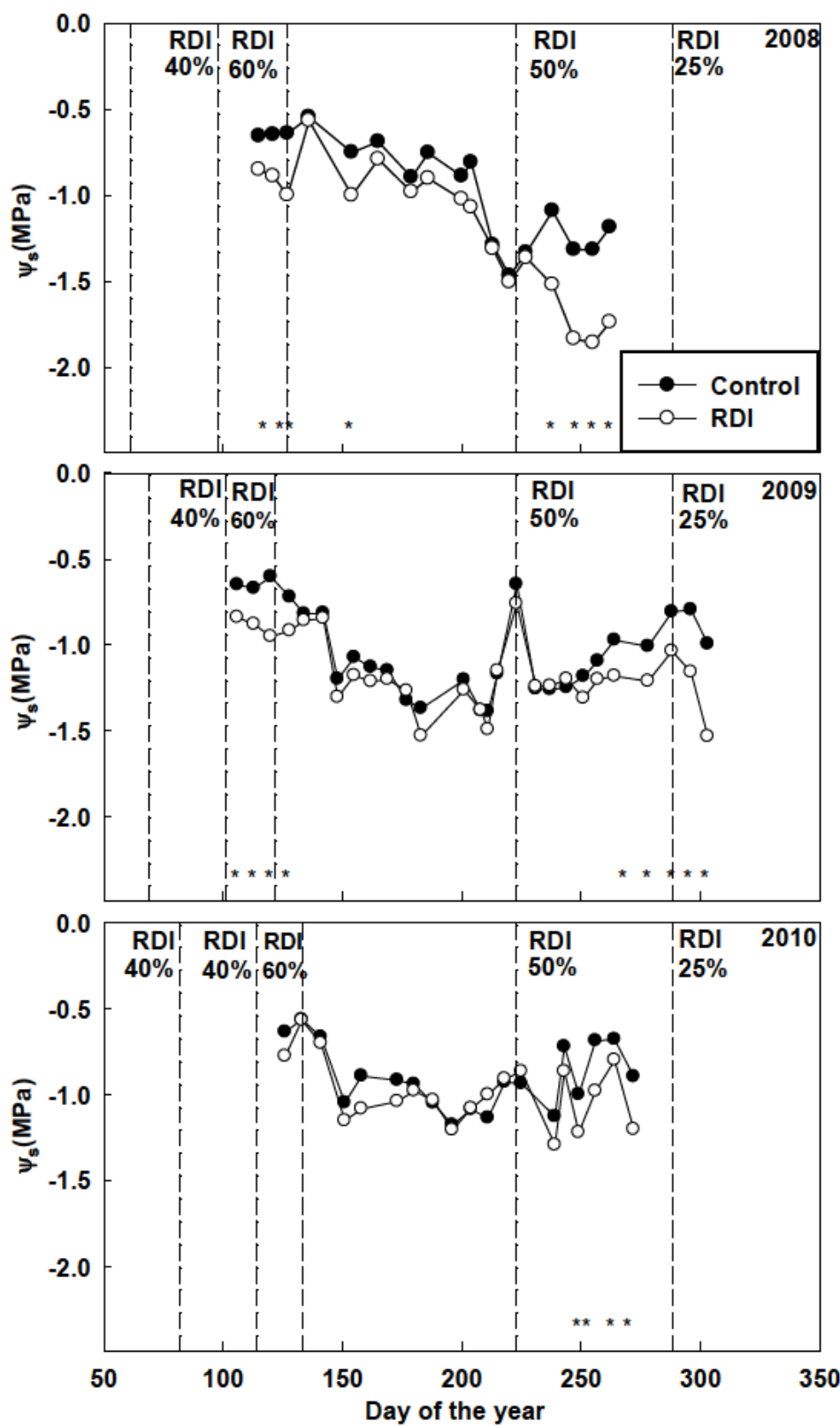

Figure. 8.5. Midday stem water potential in C (closed circles) and RDI (open circles) trees for the three studied growing seasons. Each data point is the mean of sixteen values. The intervals between vertical lines from left to right represent the beginning of the different phenological stages for apricot trees during the experimental period. Asterisks indicate significant differences between treatments $(p<0.05)$.

The RDI treatment induced significant reductions in the average values of midday $\Psi_{\mathrm{s}}$ in all the stages during which water deficit was imposed (Table 8.2). During fruit development, the values of $\Psi_{\mathrm{s}}$ were nearly constant in C treatment and were about $-0.64 \mathrm{MPa}$ in stage II. The reduction of $\Psi_{\mathrm{s}}$ in RDI treatment during this stage was significant and about $19 \%$ respect to $\mathrm{C}$ plants at stage II of fruit development $(-0.79$ 
$\mathrm{MPa}$ ). During postharvest period, $\Psi_{\mathrm{s}}$ values in $\mathrm{C}$ plants were lower due to increased evaporative demand, reaching an average of $-1.04 \mathrm{MPa}$ and $-1.14 \mathrm{MPa}$ during early and late postharvest periods, respectively.

The decrease of $\Psi_{\mathrm{s}}$ in RDI was significant and about $14 \%$ and $30 \%$ compared to $\mathrm{C}$ plants during the first and second period of late postharvest $(-1.33$ and $-1.39 \mathrm{MPa})$, respectively. RDI showed the highest values of water stress integral (Figure 8.6), which coincides with the evolution of $\Psi_{\mathrm{s}}$, with significant differences in 2008.

However, the RDI treatment induced significant reductions in the average values of midday $\Psi_{1}$ only during stage II and the second period of late postharvest (Table 8.2).

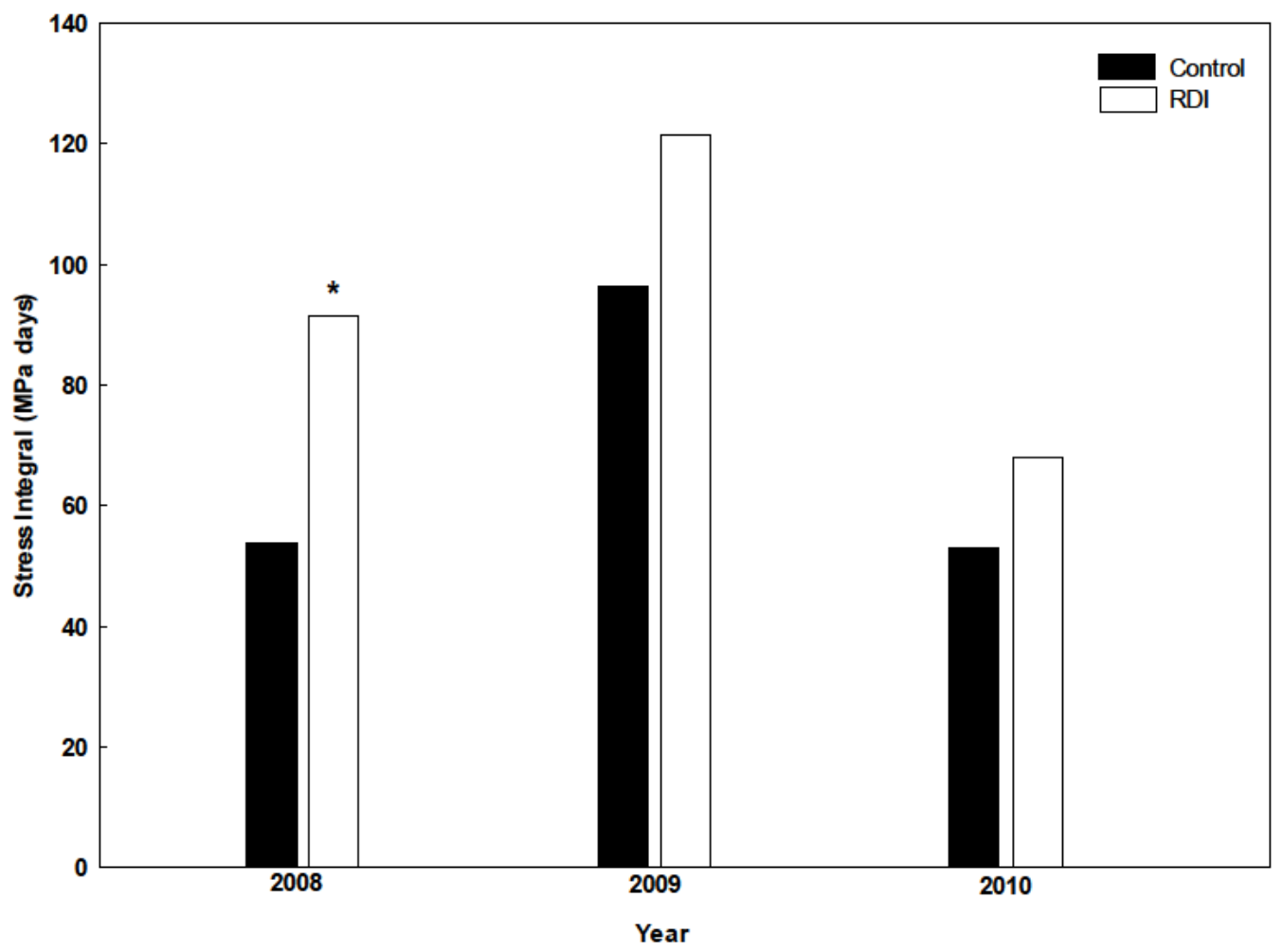

Figure. 8.6. Stress integral values for each irrigation treatment, control (black bars) and RDI (white bars) in the 2008, 2009 and 2010 growing seasons. Asterisks indicate significant differences between treatments $(p<0.05)$.

Similar behaviour to that observed in $\Psi_{1}$ was also detected for gas exchange parameters during the postharvest period, with a significant reduction in $\mathrm{P}_{\mathrm{n}}$ and $\mathrm{g}_{\mathrm{s}}$ values only at the end of the second period of late postharvest. The decrease in $\mathrm{P}_{\mathrm{n}}$ and $\mathrm{g}_{\mathrm{s}}$ for the RDI treatment was 82 and $72 \%$, respectively during this second period of late postharvest. 
Table 8.2. Average values of midday stem water potential $\left(\Psi_{s}, \mathrm{MPa}\right)$, leaf water potential $\left(\Psi_{1}, \mathrm{MPa}\right)$, net photosynthesis $\left(\mathrm{P}_{\mathrm{n}}, \mu \mathrm{mol} \mathrm{m} \mathrm{m}^{-2} \mathrm{~s}^{-1}\right)$ and stomatal conductance $\left(\mathrm{g}_{\mathrm{s}}, \mathrm{mmol} \mathrm{m} \mathrm{m}^{-2} \mathrm{~s}^{-1}\right)$ for each phenological period during the three years of experimental period, in the control (C) and regulated deficit irrigation (RDI) treatments.

\begin{tabular}{|c|c|c|c|c|c|c|c|c|c|c|c|c|}
\hline \multirow[b]{2}{*}{ 2008-2010 } & \multicolumn{3}{|c|}{$\Psi_{\mathrm{S}}(\mathrm{MPa})$} & \multicolumn{3}{|c|}{$\Psi_{1}$ (MPa) } & \multicolumn{3}{|c|}{$P_{n}\left(\mu \mathrm{mol} \mathrm{m}{ }^{-2} \mathrm{~s}^{-1}\right)$} & \multicolumn{3}{|c|}{$\mathrm{g}_{\mathrm{s}}\left(\mathrm{mmol} \mathrm{\textrm {m } ^ { - 2 } \mathrm { s } ^ { - 1 } )}\right.$} \\
\hline & Control & RDI & & Control & RDI & & Control & RDI & & Control & RDI & \\
\hline Stage I & -0.61 & -0.75 & $*$ & -1.26 & -1.45 & ns & 10.20 & 8.10 & ns & 144.10 & 107.30 & ns \\
\hline Stage II & -0.64 & -0.79 & $* * *$ & -1.24 & -1.46 & * & 8.23 & 8.12 & ns & 100.74 & 92.71 & ns \\
\hline Stage III & -0.85 & -0.98 & ns & -1.74 & -1.86 & ns & 9.42 & 9.64 & ns & 132.60 & 130.66 & ns \\
\hline Early Post-Harvest & -1.04 & -1.10 & ns & -2.22 & -2.33 & ns & 6.07 & 5.14 & ns & 78.93 & 59.75 & ns \\
\hline Late Post-Harvest I & -1.14 & -1.33 & * & -2.40 & -2.55 & ns & 6.71 & 5.14 & ns & 89.90 & 70.01 & ns \\
\hline Late Post-Harvest II & -0.98 & -1.39 & $* * *$ & -2.19 & -2.48 & * & 6.00 & 1.10 & $* * *$ & 76.60 & 22.20 & $* * *$ \\
\hline
\end{tabular}

Values are the mean of all values in the different stages. ns: non-significant. ${ }^{*} p<0.05 .{ }^{*} * * p<0.001$.

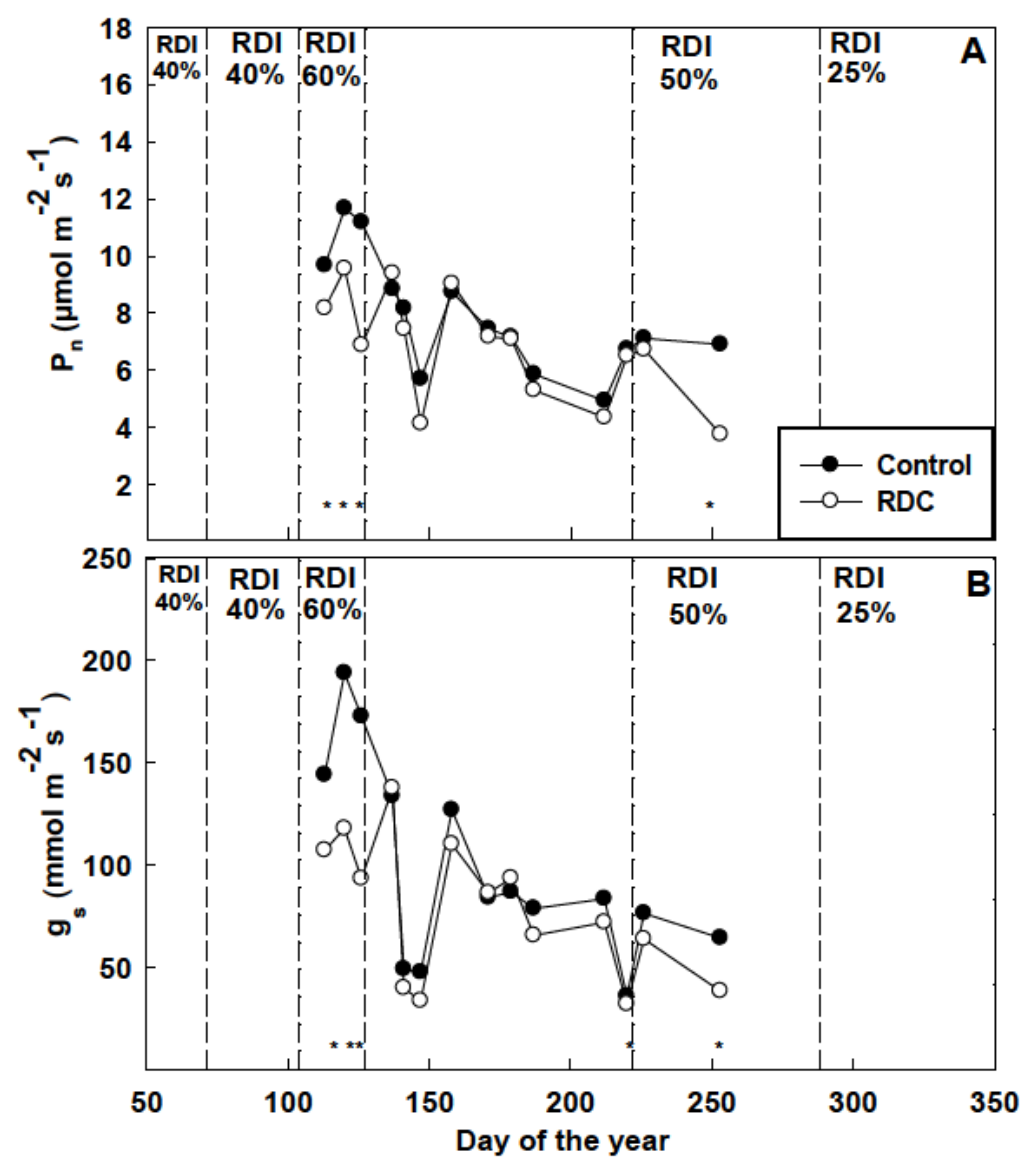

Figure. 8.7. Net photosynthesis $\left(P_{n}, A\right)$ and stomatal conductance $\left(\mathrm{g}_{\mathrm{s}}, \mathrm{B}\right)$ values averaged for the three studied growing seasons in $\mathrm{C}$ (closed circles) and RDI (open circles) trees. Each data point is the mean of sixteen values. Asterisks indicate significant differences between treatments $(p<0.05)$. The intervals between vertical lines from left to right represent the beginning of the different phenological stages for apricot trees during the experimental period.

Similar behaviour to that of $\Psi_{\mathrm{s}}$ was also observed for gas exchange parameters averaged for the three growing seasons, with a significant reduction in net 
photosynthesis $\left(\mathrm{P}_{\mathrm{n}}\right)$ (Figure 8.7A) and leaf stomatal conductance $\left(\mathrm{g}_{\mathrm{s}}\right)$ (Figure 8.7B) due to water restrictions in the RDI treatment.

When each growing season is separately considered, lower values of both $\mathrm{P}_{\mathrm{n}}$ and $\mathrm{g}_{\mathrm{s}}$ were detected in all the stages during which water deficit was imposed in 2008. In 2009 and 2010, these differences were only significant for $P_{n}$ in late postharvest in 2009 (data not shown).

\section{Vegetative and fruit growth}

Cumulative shoot growth was limited by water deficit with trees from RDI showing lower values than those observed in the control treatment (data not shown); however, this reduction was not significant. Similarly, no significant differences were observed for TCSA (Table 8.3).

However, fruit growth, measured as fruit diameter, followed a double sigmoid curve with the three characteristic stages of stone fruits (Figure 8.8). Fruits exposed to RDI had a lower but non-significant fruit diameter at the end of stage II of fruit development. When irrigation was restored in the RDI treatment, fruits rapidly reached similar diameter values to those obtained in the $\mathrm{C}$ treatment. At harvest, the fruit equatorial diameter was similar in both treatments for the three years.

\section{Yield and fruit quality}

No significant differences in yield were observed between treatments for the three growing seasons considered (Table 8.3).

Significantly lower values of fruit set were observed in RDI trees only during 2008. In contrast, water productivity was significantly higher for RDI in the three years considered in this study. Regarding water savings, the percentages were 43, 22 and 30\% respect to $\mathrm{ET}_{\mathrm{c}}$ (Control) for 2008, 2009 and 2010, respectively.

In relation to fruit quality, fruit firmness decreased significantly (30\%) in fruits from the RDI treatment only in 2008, while SSC and maturity index values were significantly increased (8.6 and $18.3 \%$, respectively) in this treatment with respect to $\mathrm{C}$ (Table 8.4).

However, there were no significant differences between treatments for $\mathrm{pH}$ and TA. In the other two years, no significant differences among treatments were detected in 
any parameter with the exception of maturity index in 2009 (12.8\% increased under RDI) (Table 8.4).
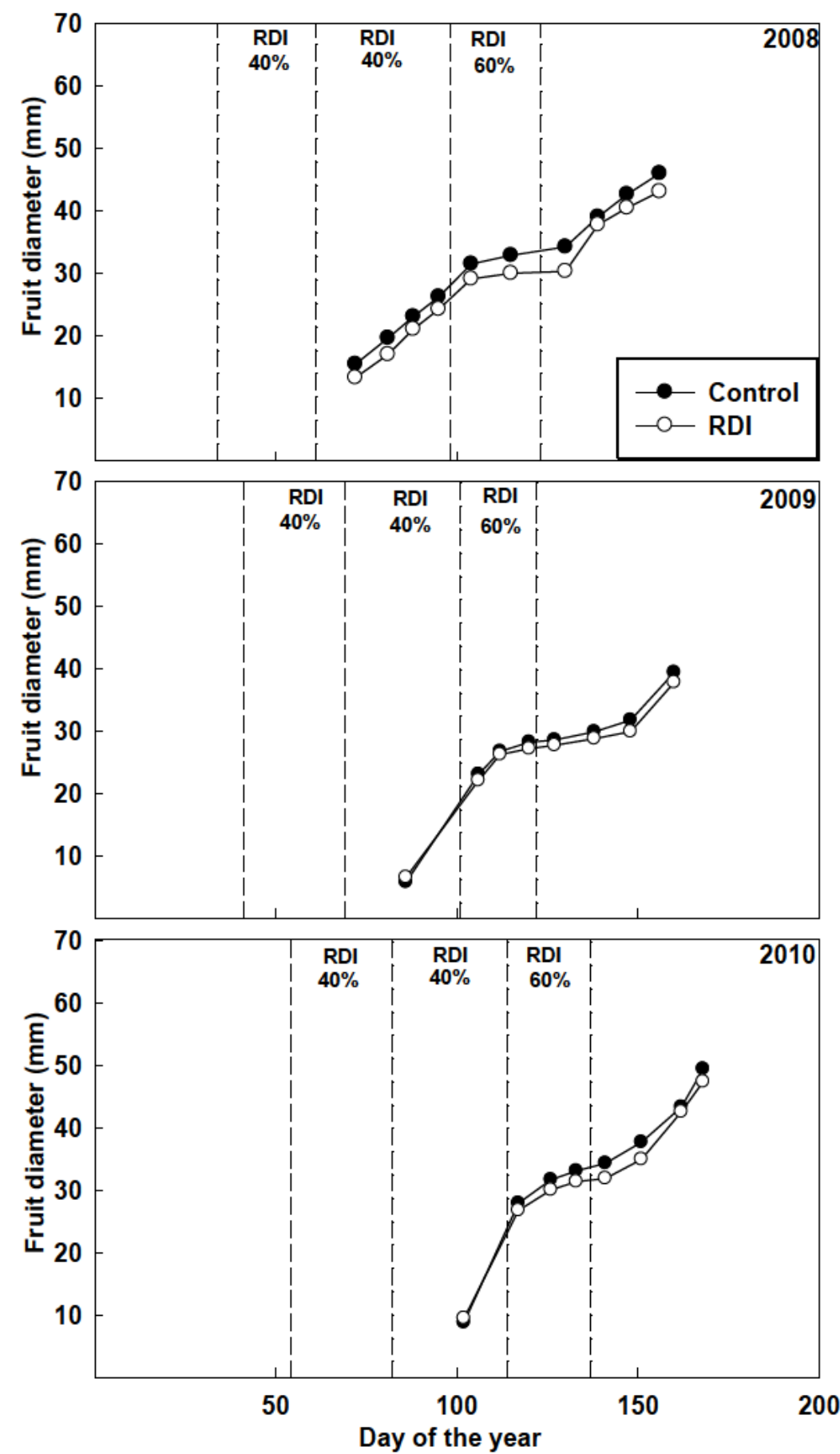

Figure. 8.8. Fruit diameter ( $\mathrm{mm})$ evolution in $\mathrm{C}$ (closed circles) and RDI (open circles) trees for the three studied growing seasons. Each data point is the mean of 200 values. The intervals between vertical lines from left to right represent the beginning of the different phenological stages for apricot trees during the experimental period. 
Table 8.3. Fruit set (\%), trunk cross sectional area $\left(\mathrm{TCSA}, \mathrm{cm}^{2}\right)$, yield $(\mathrm{kg}$ tree $^{-1}$ ), water productivity (WP, $\mathrm{kg} \mathrm{m}^{-3}$ ) and water savings as a function of the irrigation treatments for the experimental period 2008-2010.

\begin{tabular}{|c|c|c|c|c|c|}
\hline & $\begin{array}{c}\text { Fruit set } \\
(\%)\end{array}$ & $\begin{array}{l}\text { TCSA } \\
\left(\mathrm{cm}^{2}\right)\end{array}$ & $\begin{array}{c}\text { Yield } \\
\left(\mathrm{Kg} \mathrm{tree}^{-1}\right)\end{array}$ & $\begin{array}{c}\mathrm{WP} \\
\left(\mathrm{Kg} \mathrm{m}^{-3}\right)\end{array}$ & $\begin{array}{c}\text { Water savings } \\
(\%)\end{array}$ \\
\hline \multicolumn{6}{|l|}{2008} \\
\hline Control & 35.86 & 308.17 & 157 & 5.70 & \\
\hline \multirow[t]{2}{*}{ RDI } & 19.71 & 270.88 & 153 & 9.79 & 43 \\
\hline & $*$ & ns & ns & $*$ & \\
\hline \multicolumn{6}{|l|}{2009} \\
\hline Control & 37.19 & 345.45 & 104 & 4.96 & \\
\hline \multirow[t]{2}{*}{ RDI } & 38.46 & 301.33 & 106 & 6.45 & 22 \\
\hline & ns & ns & ns & $*$ & \\
\hline \multicolumn{6}{|l|}{2010} \\
\hline Control & 45.97 & 375.88 & 92 & 3.68 & \\
\hline \multirow[t]{2}{*}{ RDI } & 41.31 & 311.88 & 89 & 5.07 & 30 \\
\hline & ns & ns & ns & $*$ & \\
\hline \multicolumn{6}{|l|}{ 2008-2010 } \\
\hline Control & 39.67 & 343.17 & 118 & 4.78 & \\
\hline \multirow[t]{2}{*}{ RDI } & 33.16 & 294.68 & 116 & 7.10 & 33 \\
\hline & ns & ns & ns & $*$ & \\
\hline
\end{tabular}

The analysis of the colour values showed that the lightness factor, $L^{*}$, in flesh was similar for both treatments during the three years (Table 8.5). However, in the skin significantly higher values were observed for the RDI treatmet in 2009 and 2010.

The hue angle $\left(H^{o}\right)$ in skin and flesh was significantly higher in the fruits from the RDI treatment during 2008, whereas it was significantly greater for skin in 2009 and for the flesh in 2010. Respect to chroma $\left(C^{*}\right)$, the fruits from the RDI treatment showed significantly higher values in both skin and flesh than those from C during 2008 and 2009. These differences were not significant in 2010. 
Table 8.4. Fruit firmness (N), pH, soluble solids content (SSC, ${ }^{\circ}$ Brix), tritratable acidity (TA, g $100 \mathrm{~mL}^{-1}$ ) and SSC/TA ratio at harvest as a function of the treatment for the three experimental seasons.

\begin{tabular}{|c|c|c|c|c|c|}
\hline & $\begin{array}{c}\text { Firmness } \\
\text { (N) }\end{array}$ & $\begin{array}{c}\text { pH } \\
-\end{array}$ & $\begin{array}{c}\text { SSC } \\
\left({ }^{\circ} \text { Brix }\right)\end{array}$ & $\begin{array}{c}\text { TA } \\
\left(\mathrm{g} 100 \mathrm{ml}^{-1}\right)\end{array}$ & $\begin{array}{c}\text { Maturity Index } \\
\text { (SSC/TA) }\end{array}$ \\
\hline \multicolumn{6}{|l|}{2008} \\
\hline Control & 52.90 & 3.71 & 9.47 & 1.07 & 9.02 \\
\hline \multirow[t]{2}{*}{ RDI } & 36.50 & 3.75 & 10.28 & 0.98 & 10.67 \\
\hline & $* *$ & ns & $*$ & ns & $* *$ \\
\hline \multicolumn{6}{|l|}{2009} \\
\hline Control & 52.53 & 3.60 & 10.56 & 1.21 & 8.81 \\
\hline \multirow[t]{2}{*}{ RDI } & 49.39 & 3.62 & 11.28 & 1.17 & 9.94 \\
\hline & ns & ns & ns & ns & $* * *$ \\
\hline \multicolumn{6}{|l|}{2010} \\
\hline Control & 69.97 & 3.94 & 10.33 & 0.96 & 10.89 \\
\hline \multirow[t]{2}{*}{ RDI } & 71.83 & 3.94 & 10.53 & 0.95 & 11.15 \\
\hline & ns & ns & ns & ns & ns \\
\hline \multicolumn{6}{|l|}{ 2008-2010 } \\
\hline Control & 58.47 & 3.75 & 10.12 & 1.11 & 9.38 \\
\hline \multirow[t]{2}{*}{ RDI } & $\mathbf{5 2 . 5 7}$ & 3.77 & 10.70 & 1.06 & 10.42 \\
\hline & ns & ns & * & ns & $* * *$ \\
\hline
\end{tabular}

Table 8.5. Skin and flesh fruit colour values (reflectance measurements $L^{*}, H^{o}$, $\left.C^{*}\right)$ at harvest in the control (C) and regulated deficit irrigation (RDI) treatments, for the three experimental seasons.

\begin{tabular}{cccccccc}
\hline & & \multicolumn{2}{c}{$L^{*}$} & \multicolumn{2}{c}{$H^{\text {o }}$} & \multicolumn{2}{c}{$C^{*}$} \\
\hline \multirow{2}{*}{2008} & Control & 67.1 & 62.6 & 78.8 & 78.6 & 48.1 & 44.7 \\
& RDI & 69.2 & 63.9 & 85.3 & 82.9 & 50.6 & 46.6 \\
& & ns & ns & $* *$ & $* *$ & $* *$ & $*$ \\
2009 & Control & 64.7 & 61.4 & 71.6 & 72.5 & 44.5 & 41.2 \\
& RDI & 66.1 & 61.7 & 77.1 & 73.0 & 47.9 & 43.9 \\
& & $* * *$ & ns & $* * *$ & ns & $* * *$ & $* * *$ \\
2010 & Control & 62.7 & 59.6 & 65.4 & 65.6 & 43.4 & 40.0 \\
& RDI & 63.8 & 59.5 & 67.3 & 63.2 & 44.2 & 40.8 \\
& & $*$ & ns & ns & $* * *$ & ns & ns \\
& & 64.8 & 61.2 & 71.9 & 72.2 & 45.3 & 42.0 \\
$2008-2010$ & Control & 64.9 & Skin & Flesh & Skin & Flesh \\
& RDI & 66.4 & 61.7 & 76.6 & 73.0 & 47.6 & 43.8 \\
& & $* * *$ & ns & $* * *$ & ns & $* * *$ & $* * *$ \\
\hline
\end{tabular}

$L^{*}$ : lightness factor. $H^{o}$ : Hue value. $C^{*}$ : colour intensity (chroma). Values are the mean of 200 measurements. ns: non-significant. $* p<0.05$. ** $p<0.01$. *** $p<0.001$. 


\subsection{Discussion}

The amount of water applied in the $C$ treatment maintained high values of $\theta_{\mathrm{v}}$, close to field capacity, indicating that plants under this treatment did not suffer from water deprivation (Figure 8.3). Similar values were measured in the RDI treatment during periods of full irrigation, being the drainage low in both treatments indicating that a suitable irrigation scheduling in $C$ treatment was applied (Abrisqueta et al., 2001). Soil water profiles for the deficit irrigation treatment presented a high degree of uniformity respect to $\mathrm{C}$, from the end of April to the beginning of August. During the phenological periods of water deficit in the RDI treatment, $\theta_{\mathrm{v}}$ decreased significantly, reaching values which caused stress conditions for apricot trees (Ruiz-Sánchez et al., 2000b), especially in 2008. The higher spring rainfall amount caused that in 2010 no differences were observed between treatments during flowering and fruit set, stage I and stage II of fruit development.

The annual water savings averaged 33\% for the three-year study period and were in accordance with those reported by Pérez Pastor et al. (2009) and Pérez-Sarmiento et al. (2010) for the same cultivar. Although Pérez-Pastor et al. (2009) observed significant reductions in plant production, in our case yield was similar between treatments for the three years of the study (Table 8.3). This aspect can be explained by the highest water reductions designed by Pérez-Pastor et al. (2009) during fruit development in the initial two years (75\% until the end of stage II) in contrast with our experimental conditions $(50 \%)$, which were based on the last two years designed by those authors (Figure 8.1). Apart from this, the RDI treatment was more efficient than the control, as observed from the values of water productivity. These results are in agreement with those indicated by other authors for different fruits under water deficit conditions (e.g. Romero et al., 2004; Perez-Pastor et al., 2009).

Water savings during fruit development did not affect fruit growth in the RDI treatment, since fruits from this treatment had a slightly lower but non-significant fruit diameter at the end of the stage II of fruit development, which were more marked in 2008 (Figure 8.8). When irrigation was restored in the RDI treatment, a compensatory fruit growth was observed which allowed the fruit to reach a similar diameter to fruits from the C treatment (Chalmers et al., 1986) and at harvest apricot fruits were of «extra» size in both treatments (> $40 \mathrm{~mm}$ in diameter). This can be explained by the fact 
that fruits act as strong sinks of photosynthates, which become available when irrigation is restored, promoting higher fruit growth rates (Torrecillas et al., 2000). This behaviour has been observed in other stone fruit trees such as peach (Mitchell and Chalmers, 1982). This compensatory fruit growth during a recovery period of water deficit and the relative separation between shoot and fruit growth periods in apricot plants (Torrecillas et al., 2000) are essential for the successful application of RDI strategies (Goldhamer, 1989), indicating that deficit irrigation may be applied to limit shoot growth without detrimental effects on fruit growth and yield (Chalmers et al., 1981; Mitchell and Chalmers, 1982).

Our results showed that stress imposed did not significantly reduce vegetative growth (data not shown) and TCSA compared to trees under full irrigation conditions. The reduction of vegetative growth is of great interest for controlling canopy size and for reducing the costs associated with specific agricultural practices (Ruiz-Sánchez et al., 2010), such as pruning.

Although plant water status $\left(\Psi_{\mathrm{s}}\right.$ and $\left.\Psi_{\mathrm{l}}\right)$ and gas exchange $\left(\mathrm{P}_{\mathrm{n}}\right.$ and $\left.\mathrm{g}_{\mathrm{s}}\right)$ parameters were affected by the RDI treatment, not all these discontinuous water stress indicators performed in the same way. Thus, only $\Psi_{\mathrm{s}}$ reflected well the effects on plant water status of the different water restrictions even under mild levels of water deficit associated with low VPD (stage I of fruit growth) (Table 8.2). For this reason, the use of $\Psi_{\mathrm{s}}$ has been adopted for irrigation scheduling because of its high sensitivity to water deprivation (Remorini and Massai, 2003) and its good prediction of the yield response to deficit irrigation (Intrigliolo and Castel, 2006). The remaining water stress indicators $\left(\Psi_{1}, P_{n}\right.$ and $\left.g_{s}\right)$ depend more on the meteorological conditions (Ruiz-Sánchez et al., 2004) and in our case only presented significant differences when the water reductions in RDI treatment were very high with respect to the $\mathrm{C}$ treatment $\left(25 \% \mathrm{ET}_{\mathrm{c}}\right)$.

Despite being higher in the deficit treatment over the 3 years, the values of $S_{\psi}$ were only significant in 2008. Pérez-Pastor et al. (2014) confirmed that RDI led to yield reductions when water deficits were severe (>140 MPa-days of water stress integral) during non-critical periods. The maximum value obtained in the current study was, approximately, $120 \mathrm{MPa}$-days. For this reason, reductions in yield might have not been detected in our experiment. 
One of the benefits of RDI is an improvement in fruit taste and quality due to the increasing in SSC (Mpelasoka et al., 2000; López et al., 2011). In the present study, SSC values increased in all years for the RDI treatment although this difference was significant only in 2008. No differences were found in $\mathrm{pH}$ and tritratable acidity. Our data showed a similar fruit hardness in both treatments, with the exception of 2008 , indicated by the significant differences in firmness. Higher ratios of SSC/TA (maturity index) were observed for RDI during the three years, being significant in 2008 and 2009. This ratio affects taste perception (sweetness and acidity) by the consumer, thereby influencing buying decisions (Scandella et al., 1997). Thus, fruits from the RDI treatment can be considered of high quality since SSC increased without affecting acidity (Scandella et al., 1997).

These significant differences in fruit quality were in accordance with the previous figures in which no differences were observed neither in yield, or reproductive and vegetative growth, although the average water savings were close to $40 \%$ in the RDI treatment. These savings and the absence of impacts on fruits and trees reinforce the idea that the irrigation strategy used is optimal from an environmental and economic standpoint.

As for other parameters of fruit quality, lightness factor was significantly affected by RDI in 2009 and 2010 only for fruit skin. The $H^{o}$ has been described as a suitable and intuitively understandable colour index (Arias et al., 2000). The increase in this parameter in apricot fruits from RDI plants can be associated to a reduction in carotenoids accumulation attributed to the oxidation by exposure to light (Ruiz et al., 2005). This exposure to light in the fruits from the RDI treatment is usually related to a significant reduction in the vegetative growth of the trees during fruit development (Gelly et al., 2003; Buendía et al., 2008).

In summary, the results indicated that the apricot tree is an appropriate species to apply RDI because of its clear separation between vegetative and reproductive growths and its ability to recover the fruit diameter reduction suffered during RDI application. Furthermore, some qualitative characteristics such as the level of soluble solids, fruit taste and the colour of the fruit are enhanced. These reasons, together with average water savings of $33 \%$, emphasize the RDI strategies as a possible solution in areas with water shortage, like the south-eastern region of Spain. 


\section{Acknowledgements}

This study was supported by IRRIQUAL (EU-FP6-FOOD-CT-2006-023120) and SIRRIMED (KBBE-2009-1-2-03, PROPOSAL N॰245159) projects. We are also grateful to two SENECA projects (05665/PI/07 and 11872/PI/09) and CONSOLIDER INGENIO 2010 (MEC CSD2006-0067) and CICYT (AGL2010-17553) projects for providing funds to finance this research.

\subsection{References}

Abrisqueta J.M., Ruiz A., Franco J.A., 2001. Water balance of apricot trees (Prunus armeniaca L. cv. 'Búlida') under drip irrigation. Agricultural Water Management 50, 211227.

Allen R.G., Pereira L.S., Raes D., Smith M., 1998. Crop evapotranspiration-guidelines for computing crop water requirements. Irrigation and Drainage No. 56, FAO, Roma. 300 pp.

AOAC, 1984. Tuberculocidal activity of disinfectants. In: Oficial methods of analysis (Williams S, ed), 14th ed. Association of Official Analytical Chemists, Arlington, VA, USA. pp. 73-74.

Arias R., Lee T.C., Logendra L., Janes H., 2000. Correlation of lycopene measured by HPLC with the $L^{*}, a^{*}, b^{*}$ colour readings of a hydroponic tomato and the relationship of maturity with colour and lycopene content. Journal of Agricultural and Food Chemistry 48, 1697-1702.

Buendía B., Allende A., Nicolás E., Alarcón J.J., Gil M.I., 2008. Effect of regulated deficit irrigation and crop load on the antioxidant compounds of peaches. Journal of Agricultural and Food Chemistry 56, 3601-3608.

CARM, 2009. Anuario de Estadística Agraria. Consejería de Agricultura y Agua de la Región de Murcia. www.carm.es.

Chalmers D.J., Burge G., Jerie P.H., Mitchell P.D., 1986. The mechanism of regulation of Bartlett pear fruit and vegetative growth by irrigation with holding and regulated deficit irrigation. Journal of the American Society for Horticultural Science 111, 904-907.

Chalmers, D.J., Mitchell, P.D., Van Heek, L., 1981. Control of peach tree growth and productivity by regulated water supply, tree density and summer pruning. Journal of the American Society for Horticultural Science. 106, 307-312.

FAO, 2013. Crop statistical data webpage. http://faostat.fao.org

Fereres E., Goldhamer D.A., 1990. Deciduous fruit and nut trees. In: Irrigation of agricultural crops (Stewart B.A., Nielsen D.R., eds). American Society of Agronomy, Madison, WI, USA. pp. 987-1017.

Gelly M., Recasens I., Mata M., Arbones A., Rufat J., Girona J., Marsal J., 2003. Effects of water deficit during stage II of peach fruit development and 
postharvest on fruit quality and ethylene production. Journal of Horticultural Science and Biotechnology 78, 324-330.

Goldhamer D.A., 1989. Drought irrigation strategies for deciduous orchards. Cooperative extension. University of California, Division of Agricultural and Natural Resources. Publication no. 21453, 15 pp.

González-Altozano P., Castel J.R., 2000. Regulated deficit irrigation in 'Clementina de Nules' citrus trees II. Vegetative growth. Journal of Horticultural Science and Biotechnology 75, 388-392.

Intrigliolo, D., Castel, J.R., 2006. Performance of various water stress indicators for prediction of fruit size response to deficit irrigation in plum. Agricultural Water Management 83, 173-180.

Kijne, J.W., Barker, R., Molden, D.J., 2003. Water productivity in agriculture: limits and opportunities for improvement. Wallingford, UK: CABI, IWMI.

López G., Arbonés A., Del Campo J., Mata M., Vallverdu X., Girona J., Marsal J., 2008. Response of peach trees to regulated deficit irrigation during stage II of fruit development and summer pruning. Spanish Journal of Agricultural Research 6, 479-491.

López G., Behboudian M.H., Echeverria G., Girona J., Marsal J., 2011. Instrumental and sensory evaluation of fruti quality for 'Ryan's Sun' peach grown under deficit irrigation. Horticultural Technology 21(6), 712-719.

MAGRAMA, 2015. Anuario de estadística Avance 2014. Ministerio de Agricultura, Alimentación y Medio Ambiente.

Mitchell P.D., Chalmers D.J., 1982. The effect of reduced water supply on peach tree growth and yields. Journal of the American Socety for Horticultural Science 107, 853-856.

Moreno F., Fernández J.E., Clothier B.E., Green S.R., 1996. Transpiration and root water uptake by olive trees. Plant Soil 184, 85-96.

Moriana, A., Giron, I., Martín-Palomo, M.J., Conejero, W., Ortuño, M.F., Torrecillas, A., Moreno, F., 2010. New approach for olive trees irrigation scheduling using trunk diameter sensors. Agricultural Water Management 97, 18221828.

Mpelasoka B.S., Behboudian M.H., Dixon J., Neal S.M., Caspari H.W., 2000. Improvement of fruit quality and storage potential of "Braeburn" apple through deficit irrigation. Journal of Horticultural Science and Biotechnology 75, 615621.

Myers, B.J., 1988. Water stress integral a link between short-term stress and long term growth. Tree Physiology 4, 315-323.

Pérez-Pastor A., Domingo R., Torrecillas A., Ruiz-Sánchez M.C., 2009. Response of apricot trees to deficit irrigation strategies. Irrigation Science 27, 231-242. 
Pérez-Pastor A., Ruiz-Sánchez M.C., Domingo R., 2014. Effects of timing and intensity of deficit irrigation on vegetative and fruit growth of apricot trees. Agricultural Water Management 134 (2014) 110- 118

Pérez-Sarmiento F., Alcobendas R., Mounzer O., Alarcon J., Nicolas E., 2010. Effects of regulated deficit irrigation on physiology and fruit quality in apricot trees. Spanish Journal of Agricultural Research 2010 8(S2), S86-S94.

Remorini D., Massai R., 2003. Comparison of water status indicators for young peach trees. Irrigation Science 22, 39-46.

Romero P., Botía P., García F., 2004. Effects of regulated deficit irrigation under subsurface drip irrigation conditions on water relations of mature almond trees. Plant Soil 260, 155-168.

Roussos P.A, Sefferou V, Denaxa N.K, Tsantili E, Stathis V., 2011. Apricot (Prunus armeniaca L.) fruit quality attributes and phytochemicals under different crop load. Scientia Horticulturae 129: 472-478.

Ruiz D., Egea J., Tomás-Barberán F.A., Gil M.I., 2005. Carotenoids from new apricot (Prunus armeniaca L.) varieties and their relationship with flesh and skin colour. Journal of Agricultural and Food Chemistry 53, 6368-6374.

Ruiz-Sánchez M.C., Domingo R., Torrecillas A., Pérez-Pastor A., 2000a. Water stress preconditioning to improve drought resistance in young apricot plants. Plant Science 156, 245-251.

Ruiz-Sánchez M.C., Pérez-Pastor A., Torrecillas A., Domingo R., 2000b. Regulated deficit irrigation in apricot trees. Acta Horticulturae 537, 759-766.

Ruiz-Sánchez M.C., Torrecillas A., Nicolás E., Ortuño M.F., Abrisqueta J.M., Domingo R., Pérez-Pastor A., Alarcón J.J., 2004. Sap flow, trunk diameter and plant-water relations parameters as stress indicators of apricot trees. Acta Horticulturae 664, 575-582.

Ruiz-Sánchez M.C., Domingo R., Pérez-Pastor A., 2007. Daily variations in water relations of apricot trees under different irrigation regimes. Biologia Plantarum $51,735-740$.

Ruiz-Sanchez M.C., Domingo R., Castel J.R., 2010. Review. Deficit irrigation in fruit trees and vines in Spain. Spanish Journal of Agricultural Research 2010 8(S2), S5-S20.

Scandella D., Kraeutler E., Vénien S., 1997. Anticiper la qualité gustative des pêches et nectarines. Infos CTIFL 129, 16-19. [In French].

Scholander P.F., Hammel H.T., Bradstreet E.D., Hemingsen E.A., 1965. Sap pressure in vascular plants. Science 148, 339-346.

Soil Survey Staff. 1999. Soil taxonomy: A basic system of soil classification for making and interpreting soil surveys. 2nd edition. Natural Resources Conservation Service. U.S. Department of Agriculture Handbook 436. 
Torrecillas A., Domingo R., Galego R., Ruiz-Sánchez M.C., 2000. Apricot tree response to irrigation with holding at different phenological periods. Scientia Horticulturae 85, 201-215.

Turner N.C., 1988. Measurement of plant water status by the pressure chamber technique. Irrigation Science 9, 289-308. 
CONCLUSIONES/CONCLUSIONS 



\section{Conclusiones}

\section{Conclusiones generales}

A) Las ecuaciones de referencia realizadas con las medidas de la máxima contracción diaria (MCD) en melocotoneros adultos fueron dependientes de las variables climáticas usadas, la etapa fenológica del cultivo, así como de su carga productiva. Por tanto, estos factores deben tenerse en cuenta para ser utilizadas en la programación automática del riego por parte de los productores.

B) El riego deficitario controlado (RDC) fue programado con éxito utilizando la

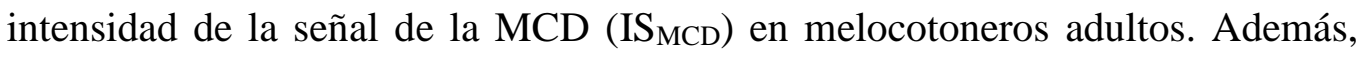
estos valores de $\mathrm{IS}_{\mathrm{MCD}}$ han demostrado su utilidad para poder determinar de manera precisa las necesidades hídricas de este cultivo.

C) El potencial hídrico de tallo a mediodía ha demostrado ser un indicador en planta del estado hídrico adecuado para poder establecer una programación del RDC en melocotonero adulto, a pesar de que sus medidas no pueden ser automatizadas.

D) Las estrategias de RDC utilizadas en el presente estudio han demostrado su utilidad para ahorrar agua (más del 30\% comparado con el riego al 100\% de la $E_{c}$ ), sin comprometer el rendimiento tanto en melocotonero como en albaricoquero. Por lo tanto, esta estrategia de riego podría suponer una solución para áreas con infradotación hídrica como es el caso del sureste español.

\section{Conclusiones parciales}

1) Las medidas de las fluctuaciones de la MCD, estuvieron mejor relacionadas con la temperatura máxima y al mediodía que con otras variables meteorológicas durante el crecimiento del fruto y, con el DPV durante el período de post-cosecha. Sin embargo, estas relaciones estuvieron muy influenciadas por las condiciones de cada año, el estado fenológico del cultivo y la carga productiva en melocotonero.

2) No se detectó una relación única entre MCD y $\Psi_{t}$ que fuera válida para todo el periodo, sino que esta relación varió cada año durante el período de crecimiento del fruto, mientras que en postcosecha las relaciones obtenidas fueron similares entre años. 
3) La $\mathrm{ET}_{0}$ diaria mostró una baja correlación con los registros de la $\mathrm{MCD}$, sugiriendo que para especies leñosas, la $\mathrm{ET}_{0}$ puede no ser el mejor criterio para determinar los requerimientos hídricos del cultivo utilizando estas ecuaciones de referencia.

4) La aplicación de estrategias de programación del riego en melocotonero a partir de valores umbrales de la $\mathrm{IS}_{\mathrm{MCD}}$ fue llevada a cabo con éxito. En este sentido, la programación del RDC no afectó la producción ni la calidad de la cosecha. Además, la reducción del crecimiento vegetativo observado se puede considerar beneficiosa ya que permite la entrada de luz en el interior del árbol y además reduce el tiempo de poda, con el consiguiente ahorro económico para el agricultor.

5) La programación del riego realizada manteniendo los valores de $\mathrm{IS}_{\mathrm{MCD}}$ cercanos a la unidad durante todo el período de cultivo nos permite cuantificar los requerimientos hídricos del cultivo y determinar, de esta manera, el coeficiente de cultivo $\left(\mathrm{K}_{\mathrm{c}}\right)$ en melocotonero.

6) Las estrategias de RDC utilizadas en este estudio fueron capaces de reducir el agua aplicada entre un 43-65\% sin afectar la producción, aumentando así la productividad del agua. Estos resultados confirmaron la posibilidad de programar el riego basándose en medidas de $\mathrm{IS}_{\mathrm{MCD}}$. Sin embargo, la sostenibilidad de estos resultados debe ser estudiada a medio-largo plazo.

7) La aplicación de estrategias de programación del riego en melocotonero a partir de valores umbrales de $\Psi_{\mathrm{t}}$ fue llevada a cabo con éxito. En este sentido, la programación del RDC no afectó la producción ni la calidad de la cosecha. y constituyen una opción viable para ahorrar agua sin comprometer la producción.

8) Desde el punto de vista de la sostenibilidad de los recursos hídricos, la programación del RDC en base a valores umbrales de $\Psi_{\mathrm{t}}$ permitió reducir de media los aportes hídricos en un 55\%, para los tres años estudiados. Sin embargo, estos ahorros fueron dependientes del valor umbral de $\Psi_{\mathrm{t}}$ utilizado, los cuales deben estudiarse más a fondo con el fin de adaptarlos a las condiciones específicas del lugar donde van a ser utilizados. 
9) El albaricoquero es una especie adecuada para aplicar estrategias de RDC debido a su clara separación entre crecimientos vegetativo y reproductivo y su capacidad para recuperar la reducción del diámetro de fruto sufrida durante la aplicación de esta estrategia de riego.

10) La producción no se vio afectada significativamente por el RDC impuesto, mientras que los parámetros de calidad mejoraron, especialmente el índice de madurez, el cual afecta a la percepción del gusto y puede influir en las decisiones de compra de los consumidores.

11) La aplicación del protocolo de RDC aplicado supuso un ahorro medio de agua del 33\%, durante el período de estudio, lo que demuestra que esta estrategia de riego puede ser una alternativa viable en áreas con escasez hídrica, como es el caso de las condiciones semiáridas del sureste de España. 


\section{Conclusions}

\section{General conclusions}

A) Reference equations established using maximum daily trunk shrinkage (MDS) measurements for adult peach trees were dependent on the climate variable used, crop phenological stages as well as crop load. Therefore, these factors must be accounted for by growers when using these equations for automated irrigation scheduling.

B) Regulated deficit irrigation (RDI) was successfully sheduled using MDS signal intensity (MDS SI) in adult peach trees. Moreover, these MDS SI values proved useful for precisely estimating peach water requirements.

C) Midday stem water potential proved to be an adequate plant-based water status indicator to establish RDI schedulings in adult peach trees, although its measurements cannot be automated.

D) The RDI strategies used in the current study proved useful for saving water (more than $30 \%$ water when compared to irrigation to the $100 \% \mathrm{ET}_{\mathrm{c}}$ ) without compromising yield in both apricot and peach trees. Therefore, this irrigation strategy could be a solution in areas with water shortage, such as the southeastern region of Spain.

\section{Partial conclusions}

1) The MDS fluctuations measured were better related to midday and maximum temperature than to other meteorological variables during fruit growth; and to VPD during the postharvest period. However, these relationships were greatly affected by the conditions of each year, tree phenological stage and crop load in adult peach trees.

2) There was not a single unique relationship between MDS and $\Psi_{\mathrm{s}}$ that was valid for the whole period, but this relationship varied each year during the period of fruit growth, while at postharvest, the relations obtained were similar between years.

3) Daily $\mathrm{ET}_{0}$ showed the poorest correlation with the MDS records suggesting that, for woody species, $\mathrm{ET}_{0}$ would not be the best criterium for determining crop water requirements by using reference equations. 
4) The feasibility of rrigation scheduling in peach based on the MDS SI thresholds was demonstrated. In this sese, the irrigation scheduling used in this study did not affect yield and fruit quality. In addition, the reduction of vegetative growth observed under the RDI strategy can be considered beneficial because it allows light to enter inside the tree and reduce pruning time, with the consequent economic savings for the farmer.

5) The irrigation strategy of maintaining MDS SI values close to unity during peach crop growth allowed for quantifying crop water requirements and thus to determine the crop factor $\left(\mathrm{K}_{\mathrm{c}}\right)$ for peach

6) The RDI strategies employed in this study were able to reduce the seasonal water applied around 43-65\% without affecting yield components and thus increasing water productivity. These results confirmed the possibility of irrigation scheduling based on MDS SI. Nevertheless, sustainability of these results must be studied at the medium-long term.

7) The irrigation scheduling strategies application in peach from $\Psi_{\mathrm{s}}$ threshold values was carried out successfully. In this sense RDC scheduling did not affect the production and quality of the harvest and constitute an option viable to save water without compromising production.

8) From the sustainability of water resources viewpoint, RDI scheduling based on $\Psi_{\mathrm{s}}$ thresholds, allowed to reduce water consumption around 55\%, in average for the three studied years. However, these water savings were dependent on the $\Psi_{\mathrm{s}}$ threshold used, which must be further explored in order to adapt them to the specific conditions of the site where they will be used.

9) Apricot tree is an appropriate species to apply RDI because of its clear separation between vegetative and reproductive growths and its ability to recover the fruit diameter reduction suffered during RDI application.

10) Fruit yield was not significantly affected by the RDI imposed, whereas fruit quality attributes improved, especially the maturity index which affects taste perception and may influence consumer's buying decisions. 
11) The RDI protocol applied supposed average water savings of 33\%, during the study period, indicated that the RDI strategy used in the current experiment may be a solution in areas with water shortage, such as the semi-arid conditions of the southeast of Spain. 
ANEXOS/ANNEXES 



\title{
10. Anexos/Annexes
}

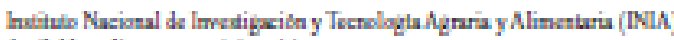
Amalable online ai wwiniacsinje

\section{Effects of regulated deficit irrigation on physiology and fruit quality in apricot trees}

\author{
F. Perez-Sarmiento ${ }^{1}$, R. Alcobendas ${ }^{1}$, O. Mounzer ${ }^{1}$, \\ J. Alarcon ${ }^{1,2}$ and E. Nicolas ${ }^{1,2 *}$ \\ ${ }^{I}$ Departamento de Riego. Centro de Edafologia y Biologia Aplicada del Segura (CEBAS-CSIC). \\ Apdo. 164. 30100 Espinardo (Murcia). Spain \\ ${ }^{2}$ Unidad Asaciada al CSIC de Horticultura Sastenible en Zonas Aridas (UPCT-CEBAS). \\ Paseo Alfanso XIII, s/n. 30203 Cartagena (Murcia). Spain
}

\begin{abstract}
The effects of regulated deficit irrigation (RDI) were studied on 9 year-old apricot-trees (Prunus armeniaca L. cv. 'Bulida') grafted on 'Real Fino' rootstock. Two irrigation treatments were established. The first, a control treatment, was irrigated to fully satisfy the crop water requirements (100\% ETC) and the second, a RDI treatment, was subject to water shortage during the non-critical periods of crop development, by reducing the amount of applied irrigation water to: a) $40 \%$ of ETc from flowering until the end of the first stage of fruit growth; b) $60 \%$ of ETc during the second stage of fruit growth and c) $50 \%$ and $25 \%$ of ETc during the late postharvest period (that starts 60 days after harvesting), for the first 30 days and until the end of tree defoliation, respectively. The results indicated that the apricot tree is an appropriate species to apply RDI thanks to the clear separation between their vegetative and reproductive growths and its ability to recover the fruit diameter reduction suffered during RDI application. Furthermore, some qualitative characteristics such as the level of soluble solids, fruit taste and the colour of the fruit are enhanced. These two reasons, together with irrigation water savings of $39 \%$, emphasize the RDI strategies as a possible solution in areas with water shortages, like the south-eastern region of Spain.
\end{abstract}

Additional key words: fruit growth, photosynthesis; Prunus armeniaca L.; regulated deficit irrigation; stomatal conductance; water relations; water stress.

\section{Resumen}

\section{Efectos del riego deficitario controlado sobre la flslologia y la calldad de frute en albaricoquero}

Se estudiaron los efectos de estrategias de riego deficitario controlado (RDC) sobre albaricoqueros de 9 anos de edad (Prunus armeniaca L. cv. 'Bülida') injertados sobre patrón franco de 'Real Fino'. Para ello, se establecieron 2 tratamientos de riego, uno de riego control, que se regó satisfaciendo los requerimientos hidricos del cultivo ( $100 \%$ ETc) y un tratamiento de RDC, que consistió en reducir los aportes de agua con respecto a la ETC en los periodos no criticos en este cultivo: a) $40 \%$ de la ETc desde floración hasta el final de la primera fase de crecimiento del fruto; b) $60 \%$ de la ETc durante la segunda fase de crecimiento del fruto y c) $50 \%$ y $25 \%$ durante la post-cosecha final ( 60 dias después de recolección), diferenciando 2 periodos de 30 dias, el primero al $50 \%$ de la ETc, y otro hasta la caida de hojas al $25 \%$ de la ETc. Los resultados obtenidos indican que el albaricoquero es una especie adecuada para realizar estrategias de RDC, debido principalmente a la clara separación entre el crecimiento vegetativo y reproductivo y, también al efecto de crecimientos compensatorios que se producen en los frutos que han estado bajo RDC, lo cual hace que finalmente no se produzcan mermas significativas de calibre. Ademas, ciertas caracteristicas cualitativas, como el nivel en solidos solubles, sabor y coloración de la fruta, se ven incrementadas. Estas dos razones, junto con ahorros de agua via riego, del $39 \%$, conducen a que estrategias de RDC puedan suponer una clara alternativa en zonas con infradotación hidrica, como es el sureste espanol.

Palabras clave adiclonales: conductancia estomatica; crecimiento del fruto; estrés hidrico; fotosintesis; Prunus armeniaca L; relaciones hidricas; riego deficitario controlado.

\footnotetext{
- Corresponding author: emilio a cebas.csic.es Received: 20-10-09; Accepted: 14-06-10.
}

Abbreviations used: DOY (day of year), ETc (crop evapotranspiration), ETo (reference crop evapotranspiration), SSC (soluble solids content), TA (tritable acidity), TDR (time-damain-reflectrometry), VPD (vapour pressure deficit). 


\section{Introduction}

Apricot trees (Prunus armeniaca L.) are widely cultivated in Mediterranean countries, with the Murcia Region being Spain's leading apricot producer with an average annual yield in the last few years of about 85,000 tonnes of apricots, from a cultivation area of 10,500 ha (MARM, 2009); these figures represent $-66 \%$ and $-60 \%$ of the Spanish total apricot production and cultivation area of apricots, respectively. The most important cultivar of apricot in Spain is 'Bullida', which represents $\sim 50 \%$ and $\sim 66 \%$ of the total production of apricots in Spain and the Murcia Region, respectively (CARM, 2009).

Apricot trees are highly sensitive to drought stress at particular phenological stages, such as stage III of fruit growth and during the 2 months after harvest (early postharvest) (Torrecillas et al., 2000; PérezPastor et al., 2007, 2009). Apricot drought tolerance is mainly based on avoidance mechanisms, such as stomatal control, epinasty and limitation transpiration by reducing leaf area (Torrecillas et al., 1999; RuizSánchez et al., 2000a), together with some degree of osmotic adjustment in young apricot trees although this adjustment is not observed in adult trees (RuizSánchez et al., 2007).

Regulated deficit irrigation (RDI) is the practice of reducing applied water at selected phenological stages less sensitive to water deficit, thus imposing plant water stress in a controlled manner, and can be a feasible water saving practice for arid areas with a minimum impact on yield and fruit quality (Chalmers et al., 1981; Goldhammer, 1989; Naor, 2006). The success of RDI strongly depends on the appropriate use of microirrigation techniques, which allows the control of soil water content (Dichio et al ., 2007). RDI has been used successfully maintaining yield and fruit quality in many fruit species (Ebel et al., 1995; Girona et al., 2003; Buendía et al., 2008; López et al., 2008), nut species (Goldhamer et al., 2000; Goldhamer and Beede, 2004; Romero et al., 2004; Girona et al., 2005) citrus species (Sánchez-Blanco et al., 1989; Castel and Buj, 1990; Domingo et al., 1996; Gonzallez-Altozano and Castel, 1999, 2000; Goldhamer and Salinas, 2000), wine grapes (Bravdo and Naor, 1996; McCarthy et al., 2002) and olives (Moriana et al., 2003). The most important research studies related to the application of RDI strategies in apricot trees has been done by RuizSanchez et al. (1999, 2000b, 2004, 2007), and PérezPastor et al. (2004, 2007, 2009). These authors have found benefits such as higher values of total soluble solids, tritratable acidity and hue angle in apricots obtained from RDI strategies (Pérez-Pastor et al., 2007).

The aim of this paper was to evaluate the effect of RDI on plant-water relations, yield and fruit quality in adult apricot trees (Primus armeniaca L. cv. 'Búlida'). The RDI treatment was scheduled to reduce water applications during non-critical periods, satisfying the crop water requirements during the critical periods, which corresponded to the second rapid fruit growth period (stage III) and the early postharvest period (Pérez-Pastor et al., 2009).

\section{Material and methods}

\section{Experimental conditions and plant material}

The experiment was performed during 2008, in a 1-ha plot of a commercial orchard, located in Mula valley, Murcia, Spain $\left(37^{\circ} 55^{\prime} \mathrm{N}, 1^{\circ} 25^{\prime} \mathrm{W}, 360 \mathrm{~m}\right.$ above sea level). The soil is a clay-loam texture and classified as a Xeric Torriorthent. It is highly calcareous, has a $\mathrm{pH}$ of 7.8, and a low organic matter content and cationic exchange capacity. The available water capacity is about $0.31 \mathrm{~m}^{3} \mathrm{~m}^{-1}$. The climate is semiarid Mediterranean with hot and dry summers; annual evaporation calculated from reference crop evapotranspiration $\left(\mathrm{ET}_{6}\right)$ and rainfall was 1,055 and $318 \mathrm{~mm}$, respectively.

The plant material consisted of 9-year-old apricot trees (Prumus armeniaca L. cv. Búlida, on Real Fino apricot rootstock), spaced $8 \times 6 \mathrm{~m}$, with an average height of $3.9 \mathrm{~m}$, trunk diameter of $0.19 \mathrm{~m}$, and ground cover of about $65 \%$. Trees were drip irrigated using one drip irrigation line for each row, with five emitters per tree, each with a flow rate of $4 \mathrm{~L} \mathrm{~h}^{-1}$.

Crop irrigation requirements were scheduled weekly according to daily ETo, calculated using the PenmanMonteith equation (Allen et al., 1998), and a local crop factor based on the time of the year (Abrisqueta et al., 2001): 0.5 February, 0.75 March, 0.8 April, 0.9 May, 0.6 June, 0.5 July-November. The correction coefficient for ground cover was 1 according to Fereres and Goldhmaer (1990). All trees received the same quantity of nutrients through the irrigation system: $110 \mathrm{~kg} \mathrm{~N}$, $62 \mathrm{~kg} \mathrm{P}_{2} \mathrm{O}_{3}$ and $117 \mathrm{~kg} \mathrm{~K}_{2} \mathrm{O} \mathrm{ha}^{-1}$ year ${ }^{-1}$. Pest control was that commonly used by growers, and no weeds were allowed to develop within the orchard.

A total of 192 trees were used in this study. The experimental design of each irrigation treatment was 
4 standard experimental plots distributed randomly in blocks. The standard plot was made up of 24 trees, organized in 4 adjacent rows. The 8 central trees of the middle row were used for measurements, and the other 16 trees were guard trees.

\section{Irrigation treatments}

Two irrigation treatments were applied: (C), irrigated daily satisfying the estimated crop evapotranspiration (ETc) and RDI irrigated at $100 \%$ ETc during the critical periods (stage III of fruit growth and 2 months after harvest period) and was subjected to water shortage during the non-critical periods of crop development by reducing the amount of applied irrigation water to: a) $40 \%$ of ETc from flowering until the end of the first stage of fruit growth; b) $60 \%$ of ETc during the second stage of fruit growth and c) $50 \%$ and $25 \%$ of ETc during the late postharvest period (that starts 60 days after harvesting), for the first 30 days and until the end of tree defoliation, respectively (Fig. 1). This distribution of water applied during noncritical periods was based on studies by Torrecillas et al. (2000). The irrigation water was considered to be of good quality with a very low electrical conductivity $\left(0.6 \mathrm{dS} \mathrm{m} \mathrm{m}^{-1}\right)$. Irrigation was controlled automatically by a head unit programmer and the amounts of water applied for each irrigation treatment were measured with in-line flowmeters placed in each standard experimental plot.

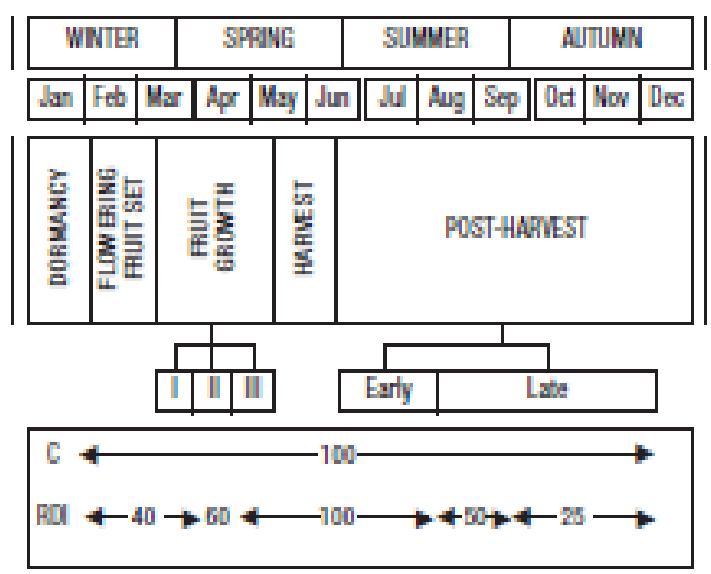

Figure 1. Percentages of crop evapotranspiration (ETc) applied in the regulated deficit irrigation (RDI) treatment during the phenological stages for 'Bülida' apricot trees during the experimental period.

\section{Measurements}

The soil volumetric water content $\left(\theta_{\text {, }}\right)$ of the top $0.2 \mathrm{~m}$ of the soil profile was measured by time-domainreflectrometry probes (TDR) (model 1502C, Tektronix Inc., OR.), as described by Moreno et al. (1996). The $\theta$, content of the soil from $0.2 \mathrm{~m}$ down to a maximum depth of $1.0 \mathrm{~m}$ was measured every $0.1 \mathrm{~m}$ using a neutron probe (model 4300, Troxler Electronic Laboratories, Inc. NC.), in access tubes installed $1.0 \mathrm{~m}$ away from the trees and beside the emitters. Measurements using one neutron probe per each standard experimental plot ( 4 per treatment) were taken every 7 to 15 days in the morning, during the experimental period. Two TDR probes were used for each neutron probe.

Midday (12:00 h solar time) stem water potential $\left(\Psi_{n}\right)$ was measured in one mature leaves per plant (6 trees per each experimental plot), taken close to the trunk. Leaves were enclosed in a small black plastic bag covered with aluminium foil for at least $2 \mathrm{~h}$ before measurements were made with a pressure chamber (Soil Moisture Equip. Corp, model 3000, Santa Barbara, CA, USA). Leaf water potential $\left(\Psi_{1}\right)$ was measured in the same trees used for $\Psi_{x}$ measurements, sampling one sunny mature leaves per plant. The water potential measurements were made according to Scholander et al. (1965) and following the recommendations of Turner (1988).

The gas exchange parameters (net photosynthesis, $\mathrm{P}_{\mathrm{n}}$ and stomatal conductance, $\mathrm{g}_{\mathrm{n}}$ ) were measured at solar midday, in a similar number and type of leaves, and in the same days and trees respectively, as for leaf water potential readings, using a field-portable photosynthesis system (LI-6400, Li-Cor, Lincoln, NE, USA).

Fruit growth was measured perpendicular to the fruit suture on 200 fruits in each treatment ( 50 fruits per experimental plot). Each sampling was carried out every $7-10 \mathrm{~d}$ from a fruit diameter minimum of $1 \mathrm{~cm}$ and sampling randomly 10 fruits in the canopy of 5 trees per experimental plot using digital callipers.

At harvest, 200 fruits in each treatment ( 50 fruits per experimental plot) were selected for their quality assessment. Skin and flesh colour, firmness, tritable acidity (TA), pH, and soluble solids content (SSC) were evaluated as quality indices. Colour values, on the surface (ground skin colour) and after peeling in the flesh, were measured with a Minolta chromameter (CR-300, Minolta, Ramsey, NJ) tristimulus colour analyzer calibrated with a white porcelain reference plate. The colour space coordinates $L^{*}, a^{*}$, and $b^{*}$, hue angle $\left[\mathrm{H}^{*}-\operatorname{arctg}\right.$ 
$\left.\left(a^{*} / b^{*}\right)\right]$, and chroma $\left(a^{* 2} / b^{* 2}\right)^{1 / 2}$ were determined around the equatorial region in three different positions (with an average of nine times for each apricot). Fruit firmness was evaluated by compression test using a Lloyd instrument (model LR10K, Fareham Hants, UK) equipped with two flat plates $\left(12 \times 18 \mathrm{~cm}^{2}\right)$. The maximum force required to deform the fruit $5 \mathrm{~mm}$ at a speed of $25 \mathrm{~mm} \mathrm{~min}^{-1}$ was determined. TA was measured by titration of $5 \mathrm{~mL}$ of juice with $0.1 \mathrm{~mol} \mathrm{~L}^{-1} \mathrm{NaOH}$ to $\mathrm{pH}$ 8.1 by an automatic titration system (AOAC, 1984). The $\mathrm{pH}$ values were measured using a $\mathrm{pH}$-meter, and SSC was determined with an Atago N1 hand-held refractometer (Tokyo, Japan).

\section{Statistical design and analysis}

Statistical analysis was performed by weighted analysis of variance (ANOVA) using general linear model of SPSS software (version 17.0, SPSS Inc., Chicago).

\section{Results}

During the experimental period the average values of volumetric soil water content $\left(\theta_{*}\right)$ from 0 to $1 \mathrm{~m}$ depth in $\mathrm{C}$ treatment was nearly constant, with values always close to field capacity $\left(95.8 \%\right.$ of $\theta_{\gamma}$ value at field capacity) (Fig. 2a). RDI treatment presented different $\theta_{\mathrm{v}}$ values ( $82.5 \%$ of $\theta$, value at field capacity) between day of year (DOY) 64 and 152 and between DOY 240 and 290, both being lower than those of C treatment (Fig. 2b). The soil moisture profile in RDI treatment was characterized by the fact that during the water deficit periods, the $\theta_{v}$ values beyond $600 \mathrm{~mm}$ were clearly below field capacity, indicating the non existence of drainage (data not shown). The amount of water applied was $574.0 \mathrm{~mm}$ and $352.4 \mathrm{~mm}$ in C and RDI treatments, respectively. The water saved in RDI treatment was $28.5 \%$ and $50 \%$ during the fruit development and late postharvest period, respectively (Fig. 2c).

Fruit growth, measured as fruit diameter, followed a double-sigmoid pattern (Fig. 3a). Fruits exposed to RDI had a lower but non-significant fruit diameter at the end of stage II of fruit development. When irrigation was restored in the RDI treatment, fruits of this treatment rapidly reached similar diameter values to those obtained in the $\mathrm{C}$ treatment due to a significant
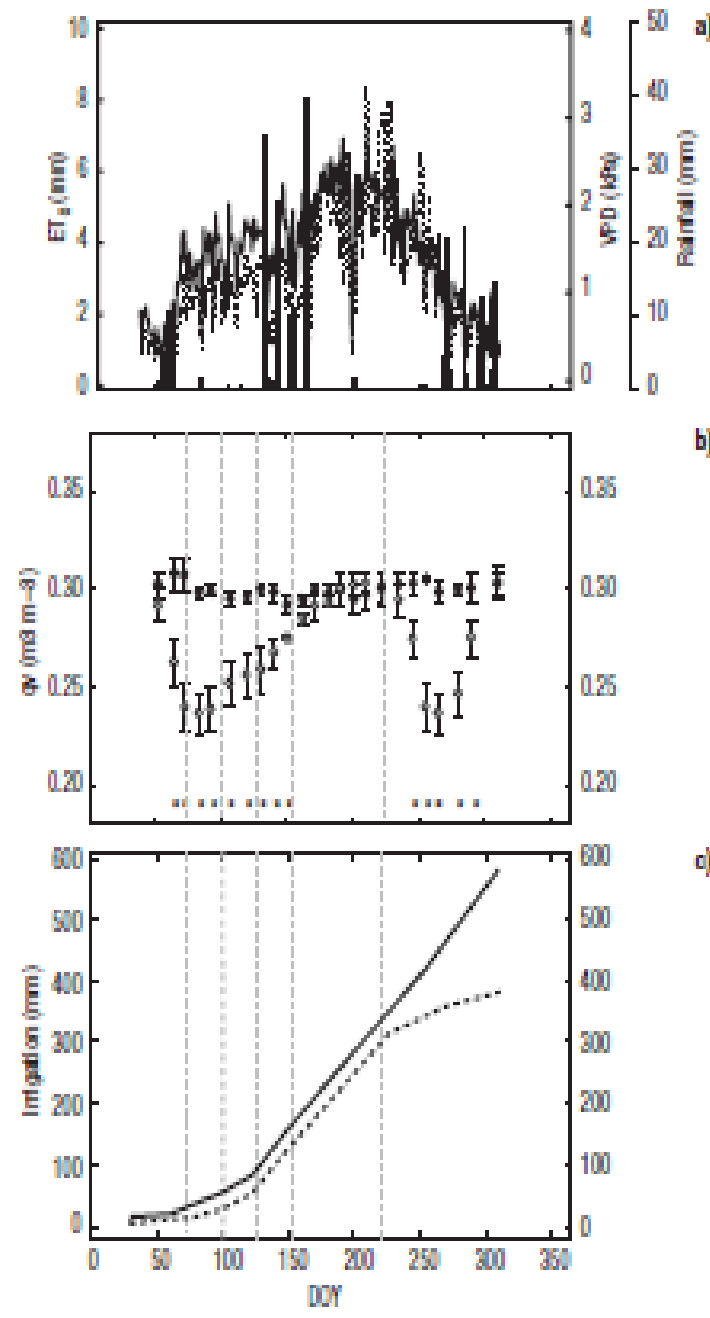

c)

Figure 2. (a) Daily crop reference evapotranspiration $\left(\mathrm{ET}_{\mathrm{l}}, \mathrm{mm}\right)$ (solid line), mean air vapour pressure deficit (VPD, kPa) (dotted line) and rainfall (mm) (vertical bars); (b) soil volumetric water content $\left(\theta_{2}\right)$ to a depth of $1 \mathrm{~m}$ in the control $(\mathbf{C})$ and RDI $(O)$ irrigation treatments; (c) cumulative irrigation water applied (mm) in the control (solid line), and RDI (discontinuous line) plants along the season (DOY-day of year). The interval between vertical dotted lines from left to right represent the beginning of phases I, II and III of fruit growth, early- and late

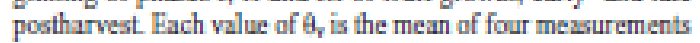
\pm SE. Asterisks indicate statistically significant differences between treatments $(p<0.001)$.

increase in the fruit growth rate (Fig. 3b). At harvest the fruit equatorial diameter was similar in both treatments and around $45 \mathrm{~mm}$ (Fig. 3a).

The RDI treatment induced statically significant reductions in $\Psi$, in all the stages during which the water deficit was imposed (Table 1). During the fruit development the values of $\Psi_{x}$ were nearly constant in $\mathrm{C}$ treatment and were about $-0.65 \mathrm{MPa}$. The reduction of 

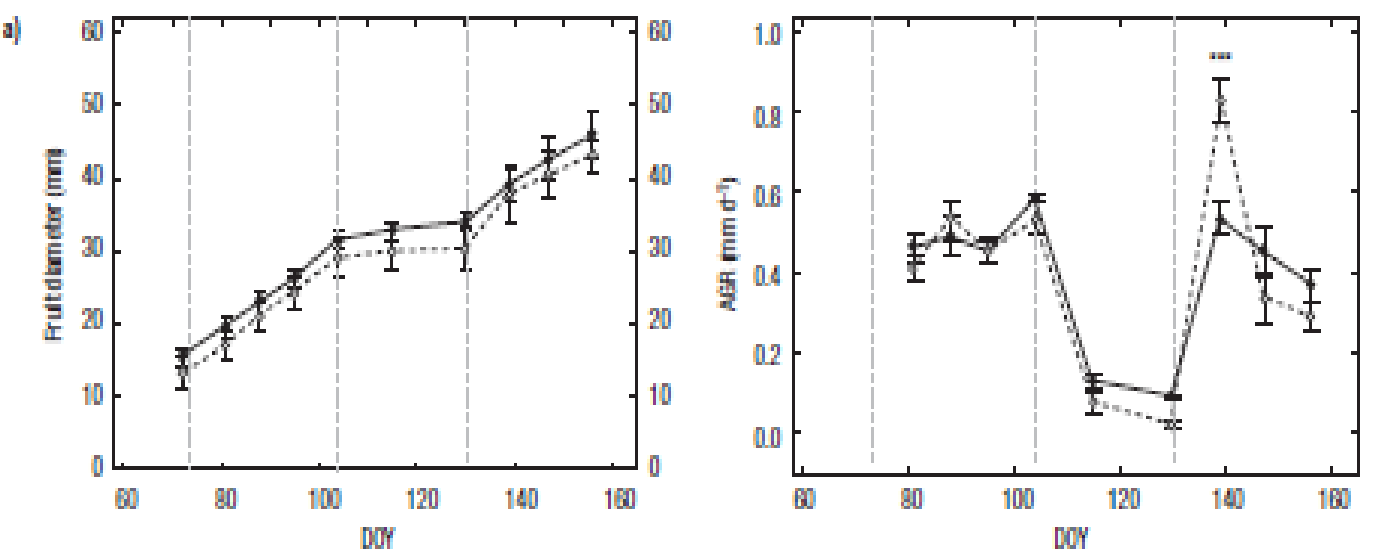

Figure 3. (a) Fruit diameter ( $\mathrm{mm}$ ) and (b) absolute fruit growth rate (AGR, $\mathrm{mm} \mathrm{d}^{-1}$ ) evolution in the $\mathrm{C}$ (solid line, $\bullet$ ) and RDI (discontinuous line, O) irrigation treatments during the fruit growth period (DOY-day of year). The interval between vertical dotted lines from left to right represent the beginning of phases I, II and III of fruit growth. Each value is the mean of 200 measurements \pm SE. Asterisks indicate statistically significant differences between treatments $(p<0.001)$.

$\Psi_{\mathrm{n}}$ in RDI treatment during this period was significant and about $47 \%$ respect to $C$ plants at the end of stage II of fruit development $(-0.94 \mathrm{MPa})$. During postharvest period, $\Psi_{1}$ values in $\mathrm{C}$ plants were lower due to increased evaporative demand, reaching an average of $-0.91 \mathrm{MPa}$ and $-1.24 \mathrm{MPa}$ during early and late postharvest periods, respectively. The decrease of $\Psi_{2}$ in RDI was significant and about of $26 \%$ and $18 \%$ compared to $\mathrm{C}$ plants during the first and second period of late postharvest respectively $(-1.55$ and $-1.81 \mathrm{MPa}$, respectively). However, RDI treatment induced statically significant reductions in $\Psi_{1}$ only during the second period of late postharvest when the water deficit was more important ( $25 \% \mathrm{ETc})$. As in the case of $\Psi_{x}$ values, $\Psi_{1}$ showed a decreasing tendency as vapour pressure deficit (VPD) increased in both treatments (Fig. 2a). The average values of $\Psi_{1}$ for both treatments were about $-1.36 \mathrm{MPa},-1.65$ and $-2.11 \mathrm{MPa}$ during fruit development, early- and late-postharvest periods, respectively. Similar behaviour to that observed in $\Psi_{1}$ was also shown in gas exchange parameters with a significant reduction in $\mathrm{P}_{\mathrm{a}}$ and $\mathrm{g}_{\mathrm{x}}$ values only being ob-

Table 1. Average values of stem $\left(\mathrm{W}_{\mathrm{n}}, \mathrm{MPa}\right)$ and leaf $\left(\mathrm{W}_{\mathrm{l}}, \mathrm{MPa}\right)$ water potential, net photosynthesis $\left(\mathrm{P}_{\mathrm{n}}, \mu \mathrm{mol} \mathrm{m}^{-2} \mathrm{~s}^{-1}\right)$ and stomatal conductance $\left(\mathrm{ge} \mathrm{mmol} \mathrm{m}^{-2} \mathrm{~s}^{-1}\right)$ in each phenological period in the control (C) and regulated deficit irrigation (RDI) treatments

\begin{tabular}{|c|c|c|c|c|c|c|c|c|}
\hline \multirow{2}{*}{$\begin{array}{c}\text { Phenological } \\
\text { period }\end{array}$} & \multicolumn{2}{|c|}{$\boldsymbol{\Psi}_{1}$} & \multicolumn{2}{|c|}{$\boldsymbol{\Psi}_{1}$} & \multicolumn{2}{|c|}{$P_{1}$} & \multicolumn{2}{|c|}{$\mathrm{g}$} \\
\hline & C & $\mathrm{RDI}$ & C & RDI & C & RDI & C & RDI \\
\hline Stage I & \multicolumn{2}{|r|}{ 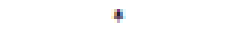 } & \multicolumn{2}{|r|}{ 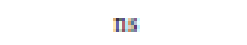 } & 10.2 & 8.1 & 144.1 & 107.3 \\
\hline Stage II & \multicolumn{2}{|c|}{4} & \multicolumn{2}{|r|}{ 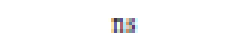 } & \multicolumn{2}{|c|}{$\mathrm{ns}$} & 167.6 & $\mathrm{~ns}$ \\
\hline Stage III & ns & -0.70 & \multicolumn{2}{|l|}{. } & $\mathrm{ns}$ & 12.7 & 196.3 & $\mathrm{~ns}$ \\
\hline $\begin{array}{l}\text { Early } \\
\text { Postharvest }\end{array}$ & \multicolumn{2}{|c|}{ ns } & \multicolumn{2}{|l|}{ 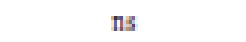 } & $\mathrm{ns}$ & 7.9 & \multicolumn{2}{|c|}{$\mathrm{ns}$} \\
\hline $\begin{array}{l}\text { Late } \\
\text { Postharvest I }\end{array}$ & -1.23 & $4 *$ & \multicolumn{2}{|c|}{ ns } & $\mathrm{ns}$ & 5.8 & \multicolumn{2}{|c|}{$\mathrm{ns}$} \\
\hline $\begin{array}{l}\text { Late } \\
\text { Postharvest II }\end{array}$ & \multicolumn{2}{|c|}{$4 * 4$} & -1.98 & -2.34 & 6.0 & 1.1 & 76.6 & 22.2 \\
\hline
\end{tabular}

Values are the mean of 24 measurements. ns: non-significant. ${ }^{*} p<0.05 . * p<0.01 . * *<<0.001$. 
Table 2. Skin and flesh fruit colour values (reflectance measurements $L^{*}, H^{*}, C^{*}$ ) at harvest in the control (C) and regulated deficit irrigation (RDI) treatments

\begin{tabular}{|c|c|c|c|c|c|c|}
\hline \multirow{2}{*}{ Treatment } & \multicolumn{2}{|c|}{$L^{*}$} & \multicolumn{2}{|c|}{$H^{*}$} & \multicolumn{2}{|c|}{$C^{*}$} \\
\hline & Skin & Flesh & Skin & Fesh & Skin & Flesh \\
\hline $\mathrm{C}$ & 67.1 & 62.6 & 78.8 & 78.6 & 48.1 & 44.7 \\
\hline \multirow[t]{2}{*}{ RDI } & 69.2 & 63.9 & 85.3 & 82.9 & 50.6 & 46.6 \\
\hline & ns & $\mathrm{ns}$ & ** & *4 & ** & $*$ \\
\hline
\end{tabular}

$L^{*}$ : lightness factor. $H^{*}$. Hue value. $C^{*}$ : colour intensity (chroma). Values are the mean of 200 measurements. ns: non-significant. ${ }^{*} p<0.05 .{ }^{* *} p<0.01$.

served at the end of the second period of late postharvest. The decrease in $\mathrm{P}_{3}$ and $\mathrm{g}_{4}$ was 82 and $72 \%$, respectively during this second period of late postharvest $R D I$ treatment. $C$ plants had average values of 11.3 and 167.6 in $\mathrm{P}_{2}$ and $\mathrm{g}_{a}$ during fruit development, respectively. These values were decreased nearly by half in the postharvest period (Table 1).

The yield and fruit number per tree were similar between treatments (156.7 and $153.5 \mathrm{~kg}^{-1} \mathrm{ee}^{-1}$ and 2,906.7 and 3,038.4 fruits per tree- ${ }^{-1}$ for $\mathrm{C}$ and RDI treatments, respectively). Irrigation treatment affected to the main quality indices of apricot fruits. The analysis of the colour values showed that the lightness factor, $L^{*}$, was similar in both treatments although slightly higher in the RDI treatment. The hue angle $\left(H^{n}\right)$ in skin and flesh was significant higher in the fruits of RDl treatment. Similar behaviour observed in $H^{\circ}$ was shown in the chroma $\left(\mathrm{C}^{*}\right)$, and the fruits from the RDI treatment showed significant higher values in skin and flesh than those obtained in C treatment (Table 2). Fruit firmness significantly decreased ( $30 \%$ ) in fruits from the RDI treatment, while SSC values were increased (9\%) significantly in this treatment with respect to C treatment. However, there were no statistically significant differences between treatments as regards $\mathrm{pH}$ and TA (Table 3).

Table 3. Fruit firmness $(\mathrm{N})$, $\mathrm{pH}$, soluble solid content (SSC,\%), tritratable acidity (TA, g $100 \mathrm{~mL}^{-1}$ ) and SSCTA ratio at harvest in the control (C) and regulated deficit irrigation (RDI) treatments

\begin{tabular}{cccccc}
\hline \multicolumn{2}{l}{ Treatment Firmness } & pH & SSC & TA & SSC/TA \\
\hline C & 52.9 & 3.71 & 9.47 & 1.19 & 7.96 \\
RDI & 36.5 & 3.75 & 10.28 & 1.14 & 9.02 \\
& $* *$ & ns & $*$ & ns & $*$ \\
\hline
\end{tabular}

Values are the mean of 200 measurements. ns: nun-significant. $* p<0.05 . * p<0.01$.

\section{Discussion}

The amount of water applied in the $\mathrm{C}$ treatment maintained high values of $\theta$, nearest to field capacity (Fig. 2b). Similar values of $\theta$, were measured in the RDI treatment during periods of full irrigation, being in both treatments the drainage low (lower than 10\%) (data not shown) indicating that a suitable irrigation scheduling in $\mathrm{C}$ treatment was applied (Abrisqueta et al., 2001). During the phenological periods of water deficit in the RDI treatment, $\theta$, decreased significantly, reaching values which provoke significant stress conditions in apricot trees (Ruiz-Sénchez et al., 2000). The annual water saving achieved in RDI treatment was $39 \%$ (Fig. 2c). This amount of water is similar to that obtained from RDI strategies during the two initial years by Pérez-Pastor et al. (2009). Although these authors observed significant reductions in plant production for both years, in our case the yield and fruit number per tree were similar between treatments. This aspect can be explained by the highest water reductions designed by Pérez-Pastor $e t$ al. (2009) during the fruit development in the initial two years (75\% until the end of stage II) in contrast with our experimental conditions (50\%) based on the last two years designed by those authors (Fig. 1).

The water saving during fruit development did not affect fruit growth in RDI treatment, since fruits from this treatment had a slightly lower but non-significant fruit diameter at the end of stage II of fruit development (Fig. 3a). When irrigation was restored in the RDI treatment, a compensatory fruit growth was observed (Fig. 3b) which allowed the fruit to reach a similar diameter to fruits from the $\mathrm{C}$ treatment (Chalmers $e t$ al., 1986) and at harvest apricot fruits were of textrai size in both treatments ( $>40 \mathrm{~mm}$ in diameter). This can be explained by the fact that fruit acts as strong sinks of photosynthates. These reserves are available when 
irrigation is restored, promoting higher fruit growth rates (Cohen and Goell, 1984; Mills et al., 1996; Torrecillas et al., 2000). This behaviour has been observed in other fruit trees such as lemon (Cohen and Goel, 1984), peach (Mitchell and Chalmers, 1982) and pear (Caspari et al., 1994). This compensatory fruit growth during a recovery period of water deficit and the relative separation between shoot and fruit growth periods in apricot plants (Torrecillas et al., 2000) is essential for the successful application of RDI strategies (Goldhamer, 1989), which indicates that deficit irrigation may be applied to limit shoot growth without detrimental effects on fruit growth and yield (Chalmers et al., 1981; Mitchell and Chalmers, 1982).

Plant water status $\left(\Psi_{2}\right.$ and $\left.\Psi_{1}\right)$ and gas exchange parameters ( $P_{n}$ and $\left.g_{n}\right)$ were affected by the RDI treatment, but not all these discontinuous water stress indicators performed in the same way. Thus, only $\Psi_{1}$ reflected well the effects on plant water status of the different water restrictions even under mild levels of water deficit associated at low VPD (stage I of fruit growth) (Table 1 and Fig. 2a). For this reason, the use of $\Psi_{x}$ has been adopted because of its high sensitivity to water deprivation (McCutchan and Shackel, 1992; Naor et al., 1995; Remorini and Massai, 2003) and its good prediction of the yield response to deficit irrigation (Naor, 2000; Intrigliolo and Castel, 2006). The remaining water stress indicators $\left(\Psi_{1}, \mathrm{P}_{\mathrm{n}}\right.$ and $\left.\mathrm{g}_{n}\right)$ depend more on the meteorological conditions (Ruiz-Sánchez et al., 2004) and in our case only presented significant differences when the water reductions in RDI treatment were very high with respect to the $\mathrm{C}$ treatment ( $25 \% \mathrm{ETc}$ ).

The water deficit imposed also affected fruit quality indices. Significant differences in fruit skin and flesh colour were found. Fruits from RDI treatment showed higher values of hue angle $\left(H^{*}\right)$ and chroma $\left(\mathrm{C}^{*}\right)(\mathrm{Ta}$ ble 2). The $H^{\circ}$ has been described as a suitable and intuitively understandable colour index (Arias et al., 2000). The increase in this parameter in apricot fruits from RDI plants can be associated to a reduction in carotenoids accumulation attributed to the oxidation by exposure to light (Ruiz et al., 2005). This exposure to light in the fruits from RDI treatment is related to a significant reduction in the vegetative growth of the trees during fruit development (data not shown), implying a high exposure to light of fruits. Similar behaviour was observed in peach fruits under RDI (Gelly et al., 2003; Buendia et al., 2008).

One of the benefits of RDI is an improvement in fruit taste and quality (Li et al., 1989; Mills et al., 1996;
Mpelasoka et al, 2000). Soluble solids content (SSC) and titratable acidity (TA) warrant particular attention due to their importance in fruit taste (Crisosto et al., 1994). In our case, SSC values were increased significantly in RDI treatment whereas TA was equal in both treatments, and therefore the SSC/TA ratio was increased significantly in RDI treatment (Table 3). This ratio affects the perception of taste (sweetness and acidity) by the consumer, thereby influencing decisions to buy again or not (Crisosto et al., 1997; Scandella et al., 1997). Thus, fruits from RDI treatment can be considered of high quality since SSC increased nearly $1^{\circ}$ without affecting acidity (Scandella et al., 1997).

In conclusion, the results indicated that the apricot tree is an appropriate species to apply RDI thanks to the clear separation between their vegetative and reproductive growths and its ability to recover the fruit diameter reduction suffered during RDI application. Furthermore, some qualitative characteristics such as the level of soluble solids, fruit taste and the colour of the fruit are enhanced. These two reasons, together with irrigation water savings of $39 \%$, emphasize the RDI strategies as a possible solution in areas with water shortage, like the south-eastern region of Spain.

\section{Acknowledgments}

This study was supported by three projects granted to the authors, IRRIQUAL (EU-FP6-FOOD-CT2006-023120), CONSOLIDER INGENIO 2010 (MEC CSD2006-0067) and SENECA (05665/PL/07).

\section{References}

ABRISQUETA LM., RUIZ A., FR.ANCO L.A., 2001. Water balance of apricot trees (Prunus armeniaca L. cv. "Búlida') under drip irrigation. Agric Water Manage 50, 211-227.

ALLEN R.G., PEREIRA L.S., RAES D., SMITH M., 1998. Crop evapotranspiration-guidelines for computing crop water requirements. Irrigation and Drainage No. 56, FAO, Roma. $300 \mathrm{pp}$.

AOAC, 1984. Tuberculocidal activity of disinfectants. In: Oficial methods of analysis (Williams S, ed), $14^{\mathbb{*}} \mathrm{ed}$. Association of Official Analytical Chemists, Arlington, VA, USA. pp. 73-74.

ARIAS R., LEE T.C., LOGENDRA L., JANES H., 2000. Correlation of lycopene measured by HPLC with the $L^{*}$, $a^{*}, b^{*}$ colour readings of a hydroponic tomato and the relationship of maturity with colour and lycopene content. J Agric Food Chem 48, 1697-1702. 
BRAVDO B., NAOR A., 1996. Effect of water regime on productivity and quality of fruit and wine. Acta Hort 427, $15-26$.

BUENDLA B., ALLENDE A., NICOLÁS E., ALARCóN L.J., GIL M.I., 2008. Effect of regulated de ficit irrigation and crop load on the antioxidant compounds of peaches. J Agric Food Chem 56, 3601-3608.

CARM, 2009. Anuario de Estadistica Agraria. Consejeria de Agricultura y Agua de la Región de Murcia. Available in: http://www.carm.es. [15 October, 2009]. [In Spanish].

CASPARI H.W., BEHBOUDIAN M.H., CHAL.MERS D.J., 1994. Water-use, growth, and fruit yield of Hosui Asian pears under deficit irrigation. J Am Soc Hort Sei 119, 383-388.

CASTEL J.R., BUJ A., 1990. Response of Salustiana oranges to high frequency deficit irrigation. Irrig Sci 11, 121-127.

CHALMERS D.J., MITCHELL P.D., VAN HEEK L., 1981. Control of peach tree growth and productivity by regulated water supply, tree density and summer pruning. J Am Soo Hort Sci 106, 307-312.

CHALMERS D.J., BURGE G., JERIE P.H., MITCHELL P.D., 1986. The mechanism of regulation of Bartlett pear fruit and vegetative growth by irrigation withholding and regulated deficit irrigation. J Am Soc Hort Sci 111, 904-907.

COHENA_ GOELL. A., 1984. Fruit development as an indicator of the irrigation needs of citrus trees. Proc Int Soc Citriculture Cong (Sao Paulo) 1, 114-118.

CRISOSTO C.H., JOHNSON R.S., LUZA J.G., CRISOSTO G.M., 1994. Irrigation regimes affect fruit soluble solids concentration and rate of water loss of ' $\mathrm{O}$ 'Henry' peaches. HortScience 29, 1169-1171.

CRISOSTO C.H., JOHNSON R.S., DEJONG T., DAY K.R., 1997. Orchard factors affecting postharvest stone-fruit quality. HortScience 32, 820-823.

DICHIO B., XILOYANNIS C., SOFO A., MONTANARO Q., 2007. Effects of post-harvest regulated deficit irrigation on carbohydrate and nitrogen partitioning, yield quality and vegetative growth of peach trees. Plant Soil 290, 127-137.

DOMINGO R., RUIZ-SANCHEZ M.C., SANCHEZBL.ANCO M.J., TORRECILLAS A., 1996. Water relations, growth and yield of Fino lemon trees under regulated deficit irrgation. Irrig Sci 16, 115-123.

EBEL. R.C., PROEBSTING E.L., EVANS R.G., 1995. Deficit irrigation to control vegetative growth in apple and monitoring fruit-growth to schedule irrigation. HortScience 30, 1229-1232.

FERERES E., GOL.DHAMER D.A., 1990. Deciduous fruit and nut trees. In: Irrigation of agricultural crops (Stewart B.A., Nielsen D.R., eds). American Society of Agronomy, Madison, WI, USA. pp. 987-1017.

GELLY M., RECASENS I., MATA M., ARBONES A., RUFAT J., GIRONA J., MARSAL J., 2003. Effects of water deficit during stage II of peach fruit development and postharvest on fruit quality and ethylene production. J Hort Sci Biotechnol 78, 324-330.
GIRONA J., MATA M., ARBONÉS A, ALEGRE S., RUFAT J., MARSAL J., 2003. Peach tree response to single and combined regulated deficit irrigation regimes under shallow soils. J Am Soc Hortic Sci 128, 432-440.

GIRONA L, MATA M., MARSAL L., 2005. Regulated deficit irrigation during the kernel-filling period and optimal irrigation rates in almond. Agric Water Manage 75 , 152-167.

GOLDHAMER D.A., 1989. Drought irrigation strategies for deciduous orchards. Cooperative extension. University of California, Division of Agricultural and Natural Resources. Publication no. $21453,15 \mathrm{pp}$.

GOLDHAMER D.A, SALINAS M., 2000. Evaluation of regulated deficit irrigation on mature orange trees grown under high evaporative demand. Proc International Society of Citriculture (ISC), IX Congress, Orlando, FL, USA. pp. 227-231.

GOL.DHAMER D.A., BEEDE R.H., 2004. Regulated deficit irrigation effects on yield, nut quality and water-use efficiency of mature pistachio trees. J Hort Sci Biotechnol $79,538-545$.

GOL.DHAMER D., FERERES E., COHEN M., GIRONA L., MATA M., 2000. Comparison of continuous and discrete plant-based monitoring for detecting tree water deficits and barriers to grower adoption for irrigation management. Acta Hort 537, 431-445.

GONZÁLEZ-ALTOZANO P., CASTEL J.R., 1999. Regulated deficit irrigation in 'Clementina de Nules' citrus trees. 1. Yield and fruit quality effects. J Hort Sci Biotechnol 74, 706-713.

GONZÁLEZ-ALTOZANO P., CASTEL J.R., 2000. Regulated deficit irrigation in 'Clementina de Nules' citrus trees II. Vegetative growth. J Hortic Sci Biotech 75, 388-392.

INTRIGLIOLO D.S., CASTEL J.R., 2006. Performance of various water stress indicators for prediction of fruit size response to deficit irrigation in plum. Agric Water Manage $83,173-180$.

LI S.H., HUGUET J.O., SCHOCH P.G., ORLANDO P., 1989. Response of peach tree growth and cropping to soilwater deficit at various phonological stages of fruitdevelopenent. J Hort Sci 64, 541-552.

LOPEZ G., ARBONÉS A., DEL CAMPO J., MATA M., VALLLVERDU X., GIRONA L., MARSAL J., 2008. Response of peach trees to regulated deficit irrigation during stage II of fruit development and summer pruning. Spanish J Agric Res 6, 479-491.

MARM, 2009. Anuario de Estadistica Agraria. Ministerio de Medio Ambiente y Medio Rural y Marino, Madrid. Available in: http://www.marm.es. [5 October, 2009].

MoCARTHY M.C., LOVEYS B.R., DRY P.R., STOLL M., 2002. Regulated deficit irrigation and partial rootzone drying as irrigation deficit irrigation for reducing agricultural water use 157 management techniques for grapevines. In: Deficit irrigation practices, FAO Water Reports No. 22. FAO, Rome, ltaly. pp. 79-87.

MeCUTCHAN H., SHACKEL K.A., 1992. Stem water potential as a sensitive indicator of water stress in prune trees. J Am Soc Hort Sci 117, 607-611. 
MILLS T.M., BEHBOUDLAN M.H., CLOTHIER B.E., 1996. Water relations, growth and the composition of 'Bracburn' apple fruit under deficit irrigation. J Am Soc Hort Sci 121, 286-291.

MITCHELL P.D., CHALMERS D.J., 1982. The effect of reduced water supply on peach tree growth and yields. J Am Soc Hort Sci 107, 853-856.

MORENO F., FERNÁNDEZ J.E, CLOTHIER B.E., GREEN S.R., 1996. Transpiration and root water uptake by olive trees. Plant Soil 184, 85-96.

MORIANA A., ORGAZ F., PASTOR M., FERERES E., 2003. Yield responses of mature olive orchard to water deficits. J Am Soc Hort Sci 123, 425-431.

MPELASOKA B.S., BEHBOUDIAN M.H., DIXON J., NEAL S.M., CASPARI H.W., 2000. Improvement of fruit quality and storage potential of 'Braeburn' apple through deficit irrigation. J Hort Sci Biotechnol 75, 615-621.

NAOR A., 2000. Midday stem water potential as a plant water stress indicator for irrigation scheduling in fruit trees. Acta Hort 537, 447-454.

NAOR A., 2006. Irrigation scheduling and evaluation of tree water status in deciduous orchards. Hort Rev 32, 111-166.

NAOR A., KL.EIN I., DORON L., 1995. Stem water potential and apple fruit size. J Am Soc Hort Sci 120, 577- 582.

PÉREZ-PASTOR A., DOMINGO R., TORRECILLAS A., RUIZ-SANCHEZ M.C., 2004. Growth and phenological stages of 'Búlida' apricot trees in south-east Spain. Agronomie 24, 93-100.

PEREZ-PASTOR A., RUIZ-SÁNCHEZ M.C., MARTINEZ LA., NORTES P., ARTÉS F., DOMINGO R., 2007. Effect of deficit irrigation on apricot fruit quality at harvest and during storage. J Sci Food Agric 87, 2409-2415.

PÉREZPASTOR A., DOMINGO R., TORRECILLAS A., RUIZ-SÁNCHEZ M.C., 2009. Response of apricot trees to deficit irrigation strategies. Irrig Sci 27, 231-242.

REMORINI D. MASSAI R., 2003. Comparison of water status indicators for young peach trees. Irrig Sci 22, 39-46.

ROMERO P., BOTIA P., GARCIA F., 2004. Effects of rogulated deficit irrigation under subsurface drip irrigation conditions on water relations of mature almond trees. Plant Soil 260, 155-168.

RUIZ D., EGEA J., TOMÁS-BARBERÁN F.A., GIL M.I., 2005. Carotenoids from now apricot (Prunus armeniaca
L.) varieties and their relationship with flesh and skin colour. J Agric Food Chem 53, 6368-6374.

RUIZ-SANCHEZ M.C., EOEA J., GALEGO R., TORRECILLAS A., 1999. Floral biology of 'Bulida' apricot trees subjected to postharvest drought stress. Ann Appl Biol 135, 523-528.

RUIZ-SÁNCHEZ M.C., DOMINGO R., TORRECILLAS A., PÉREZ-PASTOR A., 2000a. Water stress preconditioning to improve drought resistance in young apricot plants. Plant Sci 156, 245-251.

RUIZ-SÁNCHEZM.C., PERREZPASTORA., TORRECILLAS A., DOMINGO R., 2000b. Regulated deficit irrigation in apricot trees. Acta Hortic 537, 759-766.

RUIZ-SANCHEZ M.C., TORRECIL.LAS A., NICOLASS E. ORTUN̂O M.F., ABRISQUETA J.M., DOMINGO R., PÉREZ-PASTOR A., AL.ARCÓN J.J., 2004. Sap flow, trunk diameter and plant-water relations parameters as stress indicators of apricot trees. Acta Hortic 664, 575-582.

RUIZ-SANCHEZ M.C., DOMINGO R., PÉREZ-PASTOR A., 2007. Daily variations in water relations of apricot trees under different irrigation regimes. Biol Plant 51, 735-740.

SÁNCHEZ-BLANCO M.J., TORRECILL.AS A., LEÓNA., DEL AMOR F., 1989. The effect of different irrigation treatments on yield and quality of Verna lemon. Plant Soil $120,299-302$

SCANDELLA D., KRAEUTLER E., VÉNIEN S., 1997. Anticiper la qualité gustative des pêches et nectarines. Infos CTIFL 129, 16-19. [In French].

SCHOLANDER P.F., HAMMEL H.T., BR.ADSTREET E.D., HEMINOSEN E.A., 1965. Sap pressure in vascular plants. Science 148, 339-346.

TORRECILLAS A., GALECO R., PEREZ-PASTOR A., RUIZ-SANCHEZ M.C., 1999. Gas exchange and water relations of young apricot plants under drought conditions. J Agric Sci 132, 445-452.

TORRECILLAS A., DOMINGO R., GALEGO R., RUIZ SÁNCHEZ M.C., 2000. Apricot tree response to irrigation withholding at different phenological periods. Sci Hort 85, 201-215.

TURNER N.C., 1988. Measurement of plant water status by the pressure chamber technique. Irrig Sci 9, 289-308. 

